\title{
Friction stir welding (FSW) simulation using an arbitrary Lagrangian-Eulerian (ALE) moving mesh approach
}

Hua Zhao

West Virginia University

Follow this and additional works at: https://researchrepository.wvu.edu/etd

\section{Recommended Citation}

Zhao, Hua, "Friction stir welding (FSW) simulation using an arbitrary Lagrangian-Eulerian (ALE) moving mesh approach" (2005). Graduate Theses, Dissertations, and Problem Reports. 4208.

https://researchrepository.wvu.edu/etd/4208

This Dissertation is protected by copyright and/or related rights. It has been brought to you by the The Research Repository @ WVU with permission from the rights-holder(s). You are free to use this Dissertation in any way that is permitted by the copyright and related rights legislation that applies to your use. For other uses you must obtain permission from the rights-holder(s) directly, unless additional rights are indicated by a Creative Commons license in the record and/ or on the work itself. This Dissertation has been accepted for inclusion in WVU Graduate Theses, Dissertations, and Problem Reports collection by an authorized administrator of The Research Repository @ WVU.

For more information, please contact researchrepository@mail.wvu.edu. 


\title{
Friction Stir Welding (FSW) Simulation Using an Arbitrary Lagrangian-Eulerian (ALE) Moving Mesh Approach
}

\author{
Hua Zhao
}

\author{
Dissertation submitted to the \\ College of Engineering and Mineral Resources \\ at West Virginia University \\ in partial fulfillment of the requirements \\ for the degree of
}

\author{
Doctor of Philosophy \\ In \\ Mechanical Engineering \\ Victor H. Mucino, Ph.D., Chair \\ Bruce Kang, Ph.D. \\ Kenneth H. Means, Ph.D. \\ Syd S. Peng, Ph.D. \\ Jacky C. Prucz, Ph.D. \\ James E. Smith, Ph.D. \\ Department of Mechanical and Aerospace Engineering
}

Morgantown, West Virginia

2005

Keywords: Friction Stir Welding (FSW), Material Flow, Numerical Simulation, Arbitrary Lagrangian-Eulerian (ALE) Formulations, Moving Mesh

Copyright 2005 Hua Zhao 


\section{ABSTRACT \\ Friction Stir Welding (FSW) Simulation Using an Arbitrary Lagrangian-Eulerian (ALE) Moving Mesh Approach}

\section{Hua Zhao}

Material flow in the solid-state Friction Stir Welding (FSW) is quite a complex process. Investigation of material flow can be carried out either by experimentation or by numerical simulation. However, compared to experimentation, numerical simulation is inexpensive, efficient and convenient, but quite challenging to model.

This work concerns the choice and development of numerical methods for efficient and reliable simulation of the material flow during FSW. The two objectives of this work are: to develop a mesh motion scheme for simulating the large deformations of the workpieces during FSW and to assess the material flow behavior of the rigidelastoplastic problem of FSW using the moving mesh approach.

The challenging issue in modeling FSW is to deal with the large deformations of the workpiece material. The Lagrangian simulations of FSW show that the severely distorted finite elements are caused due to the large deformation of the workpiece material, which makes the Lagrangian approach inappropriate for modeling FSW. Thus, Arbitrary Lagrangian-Eulerian (ALE) formulations are used to overcome the shortcoming of Lagrangian formulations. The basic idea of the ALE approach is that the mesh is not obliged to follow material flow. Thereby the excessively distorted elements can be avoided.

An important consideration in applying the ALE approach is an advection method which determines the mesh motion in every step of the analysis. Due to the characteristics of FSW, the moving mesh approach based on ALE formulations is developed for the modeling of FSW. Several case studies that document the material flow during FSW are presented using this approach.

Based on the simulation results, it is concluded that the material motion characteristics on the top surface and through the depth (volume) of friction stir welds have been made for the advancing and retreating sides. The motion trends are consistent with the reported experimental evidence. The case studies demonstrate the capabilities and potential of the mesh motion scheme in simulating the FSW process. 


\section{TABLE OF CONTENTS}

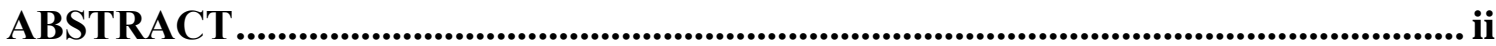

TABLE OF CONTENTS .........................................................................................iii

LIST OF FIGURES .......................................................................................................... vi

ACKNOWLEGEMENTS ................................................................................................ $\mathrm{x}$

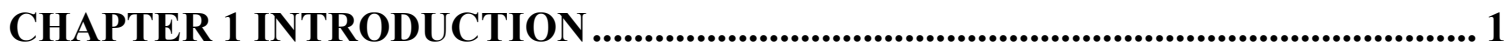







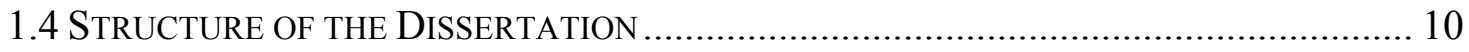

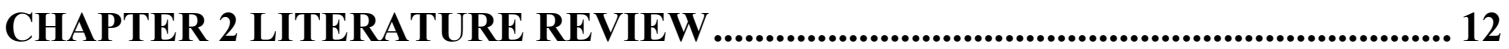

2.1 EXPERIMENTAL WORKS AND NUMERICAL STUDIES OF THE MATERIAL FLOW DURING FSW

2.2 LAGRANGIAN, EULERIAN, ALE FORMULATIONS AND THEIR APPLICATIONS IN



2.2.1 Lagrangian and Eulerian Formulations................................................... 16

2.2.2 Arbitrary Lagrangian-Eulerian (ALE) Formulations................................... 19

2.3 LITERATURE REVIEW SUMMARY ……………............................................... 31

\section{CHAPTER 3 ARBITRARY LAGRANGIAN-EULAERIAN (ALE)} FORMUALTIONS APPLIED TO THE SIMULATION ........................................... 32

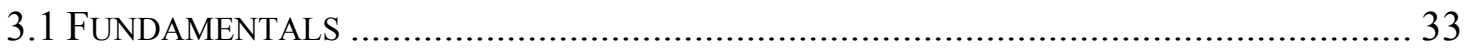



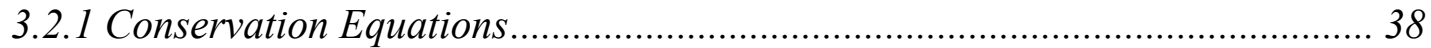

3.2.2 Constitutive Equation.............................................................................. 39

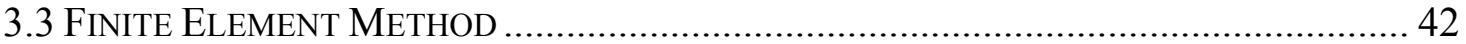



3.3.2 Finite Element Approximations ………………….................................. 43 








3.4.2 Lagrangian step ..................................................................................... 48





3.4.3.2 Remap of Solution Variables ............................................................ 53











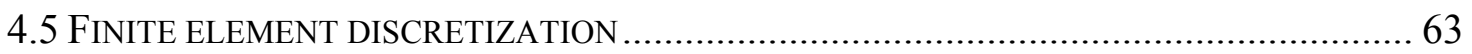





4.8 LOADING AND BOUNDARY CONDITIONS …........................................................... 70



4.9.1 Results Based on Lagrangian Formulations ............................................ 73

4.9.2 Results Based on ALE Formulations ........................................................ 76

CHAPTER 5 MOVING MESH FOR THE ALE MODELING OF FSW .................. 82

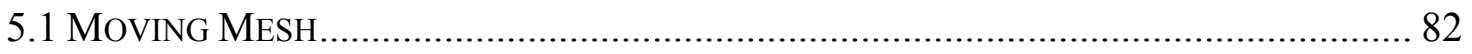

5.2 Applications of Moving MESH to the ALE Modeling OF FSW ...................... 86

5.2.1 Case One - The Material Flow in the Same Aluminum Alloy Weld................ 86

5.2.1.1 Problem Definition............................................................................... 86



5.2.1.3 Comparison of Model Flow Patterns with Experimental Data ............... 113



5.2.2 Case Two - The Material Flow in Two Material Models ........................... 119

5.2.2.1 Problem Definition............................................................................. 119

5.2.2.2 Simulation Results ............................................................................ 121

5.2.3 Case Three - The Material flow in the Dissimilar Aluminum Alloy Weld.... 125

5.2.3.1 Problem Definition.................................................................................. 125

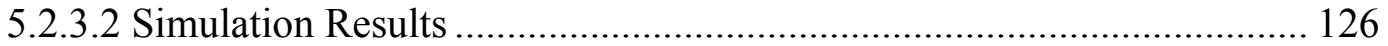

5.2.4 Case Four - Friction Effect on the Material Flow during FSW .................. 129

5.2.4.1 The Basic theory of Frictional Contact ............................................. 129

5.2.4.2 Friction Effect on Material Flow ................................................... 130

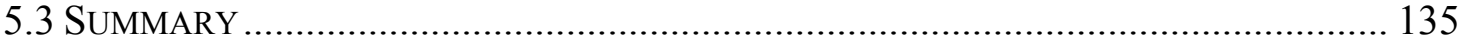

CHAPTER 6 DISCUSSION OF RESULTS .................................................... 137 
CHAPTER 7 CONCLUSIONS........................................................................................... 147



7.2 Contribution to the State of THE ART IN FSW Simulation.......................... 149









A NOMENCLATURE................................................................................................... 162 


\section{LIST OF FIGURES}

Figure 1-1 Principle of Friction Stir Welding (The Welding Institute WebPage [27]). 3

Figure 1-2 friction Stir Welding Tool (the Webpage of the Department of Materials SCIENCE \& Metallurgy, University of CAMBridge [28])....... 3

Figure 1-3 FSW Procedure for a Butt Weld (Hitachi Ltd. Webpage [29]) ............. 5

Figure 1-4 A Friction Stir Butt Weld Made of MAgnesium Alloy (Li et Al. [35]) 5

Figure 2-1 One Dimensional Example of Lagrangian, Eulerian and Ale Mesh AND MAterial Point Motion (BelytschKo ET AL. [2]) .............................. 18

Figure 2-2 Comparison of Spatial Lagrangian Mesh (left) and AlE Mesh (Right) AT DifFERENT TIME InSTANCES (HuERTA ET AL. [30])...................... 24

Figure 2-3 Variation of Time-increment, $\Delta$ T, with Time (During the Deformation PROCESS) (HUERTA ET AL. [30]) 25

Figure 2-4 Comparison between Final Yield Stress Distributions Calculated Using LAGRANGIAN AND ALE FormULATIONS (HUERTA ET AL. [30])........... 25

Figure 2-5 Pulling a Cylindrical Bar and Finite Element Discretization (LINDER [37]) .26

Figure 2-6 (a) Deformed Lagrangian Mesh and (B) Well Distributed Ale Mesh AT DiFFERENT TiME INSTANTS (LiNDER [37]) 28

Figure 2-7 Von Mises Stress Distribution in the Necking Zone at t $=20 \mu \mathrm{S}$ For LAGRANGIAN AND ALE COMPUTATION (LINDER [37]) .................................. 28

Figure 2-8 Coining Test and Finite Element Discretization (Linder [37])........... 30

Figure 2-9 Spatial Lagrangian and ALE Mesh at Different Reduction (Linder



Figure 3-1 Lagrangian Kinematic Description ..................................................... 33

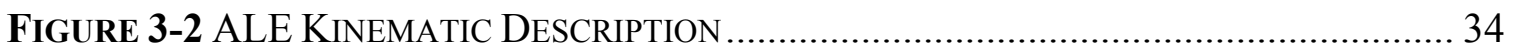


Figure 3-3 Evaluating the Angle ( $\theta$ ) and Volume (V) Around a Node (Red Point)

Figure 3-4 The Numbering of the Points ARound the Central Point (Red) For the EQUiPOTENTIAL RELAXATION STENCIL (HALlQUiST [26]).............................. 51

FiguRE 3-5 ILLUSTRATION OF THE DifFERENT $\phi_{n+1}^{L}$ 's (LINDER[37]) ................................. 56



Figure 4-2 Finite Element Discretization of the Two Workieces (Blue And RED), THE PIN (GREEN) AND SHOULDER (YELLOW) ........................................ 64

Figure 4-3 True Stress-Strain Curves of Al 6061-T6 at Various Temperatures (DENG ET AL. [10]) 66

Figure 4-4 (a) Elastic-plastic Behavior with Kinematic Hardening ( $E_{t}$ is the Slope of the Bilinear True Stress-Strain Curve); (B) ElasticPERFECTLY PlastiC MODEL. 66

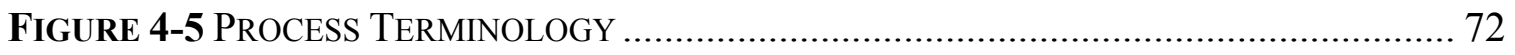

Figure 4-6 Plastic Strains in the WorkPieces at Different Time InStances ....... 75

Figure 4-7 Effective Plastic Strain Contours of the WorkPieces at Different TIME INSTANCES 77

Figure 4-8 Volume Fraction Contours and Spatial Mesh of the WorkPieces at DIFFERENT TIME INSTANCES 80

Figure 4-9 Internal and Hourglass Energies vs. Time FOR the Two WorkPieces 81

Figure 5-1 FSW Simulation with a Rotating and Translating Pin: (a) Initial

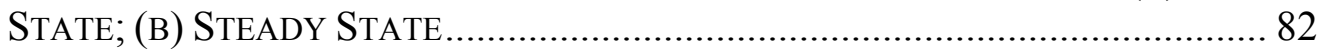

Figure 5-2 FSW Simulation Using the Moving Mesh Method: (a) Initial State;

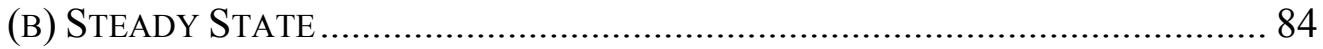

Figure 5-3 Mesh Motion of the WorkPieces in the Moving Mesh Method ........... 85



Figure 5-5 Volume Fraction Contours for the Workieces at DifFerent Time INSTANCES. 
Figure 5-6 ForTy-FOUR TRACERS AT THE TOP SURFACE OF THE WORKPIECES WITHIN THE SHOULDER DiAMETER ARE DEFINED IN THE MODEL

Figure 5-7 Planar and Vertical (Depth-wise) Motions of Tracer T1-T11 in the MODEL

Figure 5-8 X And Y CoOrdinates vs. Time (a Segment of 0 s-0.1 s) FOR Tracer T1$\mathrm{T} 11$ 103

Figure 5-9 Z COORDINATES vS. TIME FOR TRACER T1-T11 103

Figure 5-10 Planar and Vertical (Depth-Wise) Motions of Tracer T12-T22 in the MODEL 106

Figure 5-11 Z COORDINATES vS. TIME FOR TRACER T12-T22 106

Figure 5-12 Planar and Vertical (Depth-Wise) Motions of Tracer T23-T33 in the MODEL 109

Figure 5-13 Z COORDINATES vS. TIME FOR TRACER T23-T33 109

Figure 5-14 Planar and Vertical (Depth-Wise) Motions of Tracer T34-T44 in the MODEL 112

Figure 5-15 Z COORDINATES vS. TIME FOR TRACER T34-T44 112

Figure 5-16 Schematic Drawing of the Marker Insert Placement (Seidel [53])

Figure 5-17 Finite Element Predictions of the Material Flow Pattern on the TOP OF SURFACE. 116

Figure 5-18 Material Motion on the Top Surface of the Weld in FSW Test (SEIDEL [53])..... 117

Figure 5-19 Vertical Material Motion (Depth-Wise) in FSW Test (Seidel [53])117

Figure 5-20 Comparison of Volume Fraction Contours Between Two Different MATERIAL MODELS AT T $=0.2 \mathrm{~s}$ 123

Figure 5-21 Comparison of the MaXimum Effective Plastic Strain Between Two DifFERENT MATERIAL Models THROUGHOUT THE COMPUTATION 124

Figure 5-22 Mass Density Contours for the WorkPieces at Different Time INSTANCES 128 
Figure 5-23 Volume Fraction Contours for the WorkPieces With Different Frictional COEFFICIENTS AND Forging ForCE DisPlaCEMENT AT T $=0.2 \mathrm{~s}$

Figure 6-1 Particle 9 as a Tracing Point, Moving into the Whirlpool then Sticking to the Pin (A); AWAY From the PIN (B); AND SPIRAling BaCK to the Pin AT a GREater DePTH (C) 138

Figure 6-2 Particle 9 as a Tracing Point Coordinates, Moving into the WhiRlPoOl (SPIRAL1), STICKING TO THE PIN (PIN Rot. 1), AWAY FROM THE Pin (SPiral 2) and back to the Pin (Pin Rot.2) at a Greater Depth .... 139

Figure 6-3 Sequence of Trace Particle Motions at Beginning, Middle and End OF THE SIMULATION. 141

Figure 6-4 Sequence of Trace Particle Motions at Beginning, Middle and End OF THE SiMULATION. 142

Figure 6-5 Experimental Tracing of Material Motion on the ToP of the WORKPIECES BY SEIDEL ET AL. [53] 144

Figure 6-6 Experimental Tracing of Material Motion inside the WorkPiece MATERIAL (DEPTH-WISE) BY SEIDEL ET AL. [53]........................................... 144

Figure 7-1 Procedure Flowchart of the Ale Moving Mesh Approach for FSW SIMULATION 153 


\section{ACKNOWLEGEMENTS}

I extend my sincere gratitude and appreciation to all those who made this Ph.D. dissertation possible. First of all I would like to thank my research advisor, Dr. Victor H. Mucino, for the opportunity to do this research, his invaluable instructions, and his excellent feedback to written work.

My special thanks go to Dr. Syd S. Peng from the Department of Mining Engineering for his support and encouragement.

I would also like to convey my thanks and appreciation to the other committee

members, Dr. Bruce Kang, Dr. Kenneth H. Means, Dr. Jacky C. Prucz and Dr. James E. Smith, who took the time to read and provide me with valuable comments on this dissertation.

Furthermore I would like to thank my friends Qinghua Jin and Hongyan Sun for their help during the last semester.

I am very grateful for my mother, my sisters, my husband Bo and my daughters Anqi and Nova whose constant love, support and encouragement enabled me to complete this work, and also for my father, who, unfortunately, lost his battle with illness before this dissertation was completed. 


\section{CHAPTER 1 INTRODUCTION}

\subsection{Background}

Welding is a material joining process in which two workpieces are coalesced at the surfaces in contact with each other by heat, pressure, or a combination of both. Typically, welding is divided into two major groups: fusion welding and solid-state welding. Fusion welding uses heat to melt two workpieces to make a joint. Most fusion welding processes involve the addition of a filler metal which is melted into the joint. On the other hand, solid-state welding refers to the joining processes in which two workpieces are brought together under pressure, perhaps with the application of heat, to form a metallic bond across the interface. If heat is applied, the temperature in the processes is below the melting point of the workpiece material being welded so that there is no melting of the base material. In addition, no consumable filler material is normally necessary in solid-state welding. It is apparent that the main difference between those two kinds of welding techniques lies in the fact that no melting of the base material occurs in solid state welding while it does in fusion welding. The more modern solid-state welding techniques are typified by friction welding. Its basic idea is to use the friction-generated plasticized zone between two workpieces to form a joint.

Friction Stir Welding (FSW) is one application of friction welding and was invented and patented by The Welding Institute (TWI), Cambridge, UK in 1991 (Thomas et al. [57]). This significant advancement has permitted friction technology to be used to produce continuous welded seams for plate fabrication, particularly in light alloys. The basic process principle of FSW is shown in Fig.1-1, as applied to a butt joint of two 
workpieces. It is seen that two workpieces are closely butted together and a welding tool makes contact with them. A rotating velocity and a translating velocity act on the welding tool, at the same time a sufficient downward force is applied to the tool to maintain registered contact. The process relies on three factors to create a high-quality and defectfree joint: frictional heating which is generated at the tool/workpiece interface; pressure provided by the tool force; and tool rotation. The detailed FSW process is provided in the later paragraph.

The FSW tool is a non-consumable, specially shaped and made of a material that is harder than the workpiece material being welded. The tool includes a profiled pin (or probe) and a cylindrical shoulder, as shown in Fig.1-2. The pin is normally threaded. The thread can increase the frictional force between the workpieces and the pin, and help stir the plastically deformed workpiece material up around the pin, resulting in a porosityfree weld. The shoulder makes contact generating much of the heat and restricting the pin further penetration while expanding the weld zone. The pin has a diameter of approximately one third of the shoulder and a length slightly less than the thickness of the workpieces being welded.

It should be noted that the FSW process is not symmetric about the joint line: two sides of a friction stir weld are called advancing side and retreating side, respectively, as shown in Fig.1-1. The advancing side of a weld is defined as the side on which the rotational velocity vector of the welding tool has the same sense as the translational velocity vector of the tool relative to the workpieces. The retreating side is where the two vectors are of opposite sense. In addition, the leading side refers to the front of the tool and the trailing side is the back of the tool. 




Figure 1-1 Principle of Friction Stir Welding (The Welding Institute Webpage [27])



Figure 1-2 Friction Stir Welding Tool (the Webpage of the Department of Materials Science \& Metallurgy, University of Cambridge [28]) 
The detailed FSW procedure for a butt weld is illustrated in Fig.1-3. To start, the two workpieces to be welded are placed onto a rigid backing plate and clamped firmly, as shown in Fig.1-3(1). This prevents the two workpieces from moving apart. Once the workpieces are fixed, the pin slowly penetrates into the weld line at a rotational speed until the shoulder fully contacts the surface of the workpieces, as shown in Fig.1-3(2). Then the tool moves forward along the welding line in a translational speed, as shown in Fig.1-3(3). As the process proceeds, the frictional heat is generated at the tool/workpiece interface, causing the temperature in the workpiece material around the tool to increase substantially but below the melting point of the material. The increase in temperature softens the workpiece material. Then the rotating tool mechanically stirs the softened material from the front of the pin to the back, and pressure provided by the tool force consolidates the softened material to form a metallic bond. This process continues until the end of the weld is reached. The welding tool is then retracted while keeping the tool rotational speed, as shown in Fig.1-3(4). Fig.1-4 shows one example of the friction stir butt weld that is made between two same magnesium alloy workpieces. 
(1) Start



(3) Moving

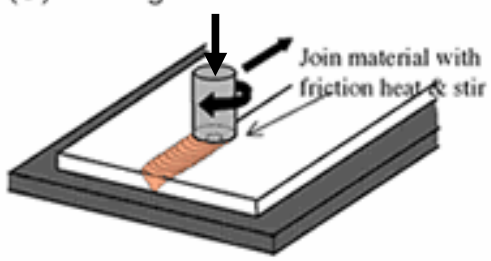

(2) Plunged into the joint

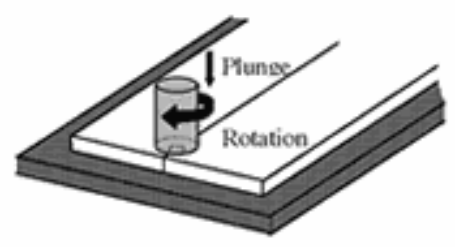

(4) Finished

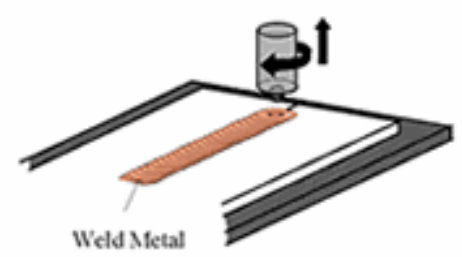

Figure 1-3 FSW Procedure for a Butt Weld (Hitachi Ltd. Webpage [29])

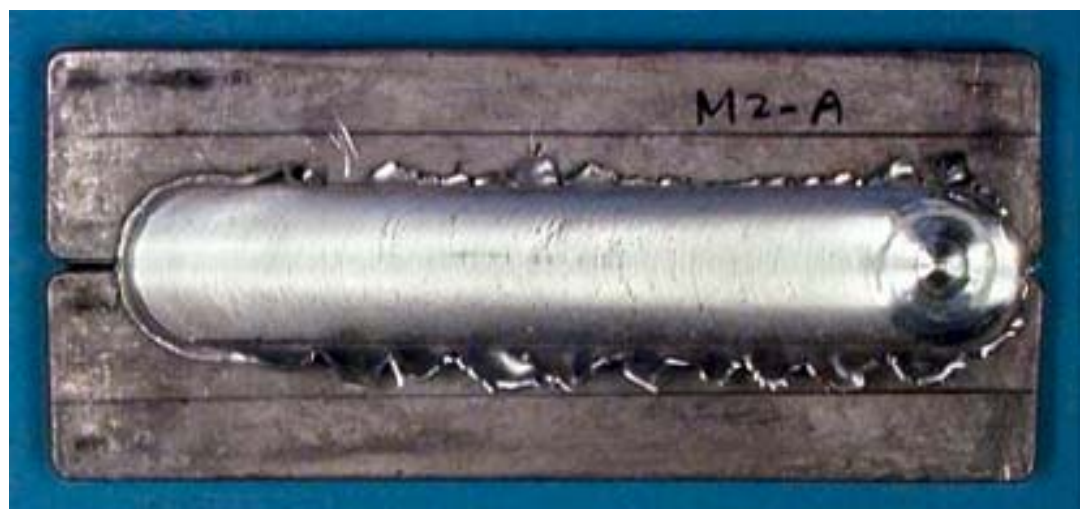

Figure 1-4 A Friction Stir Butt Weld Made of Magnesium Alloy (Li et al. [35]) 
The characteristics of FSW make it have some important advantages over fusion welding. Firstly, since no melting of the workpiece material takes place in FSW, solidification and liquation cracking and porosity are eliminated. Secondly, because melting point incompatibilities are not an issue, dissimilar and incompatible alloys thought to be unweldable with fusion welding can be joined successfully. Thirdly, there is no change in material composition since no additional material is added to the joints except for frictional heat. Fourthly, due to the lower heat input in FSW, friction stir welds exhibit the improved mechanical properties and lower residual stresses and distortions compared to fusion welds. Other advantages include no fumes, no arcs, and no splatter in FSW, which make FSW friendly to the environment. On the other hand, FSW has following disadvantages: two workpieces must be rigidly clamped to prevent two pieces from being apart; backing bar is required to react the downward force of the welding tool on the workpieces; keyhole is left at the end of each weld; and it cannot make joints which required metal deposition (e.g. fillet welds).

Due to the process characteristics, FSW is well suited for joining a large series of metals and alloys, some of which are difficult to join by fusion welding. The first applications have been in high-strength aluminum fabrication. To date, the applications of FSW to aluminum alloys have been a great success. The process can also be used to join lightweight magnesium alloys (e.g. Johnson et al. [32] and Li et al. [35]), hightemperature steel (e.g. Lienert et al. [36], Reynolds et al. [48] and Thomas et al. [58]) and other metals. In addition, this process is suited to join dissimilar material combinations such as different types of aluminum alloys (e.g. Ouyang et al. [44]), dissimilar magnesium alloys, magnesium alloy to aluminum alloy (e.g. Somasekharan et al. [54]), 
aluminum alloy to steel (e.g. Kimapong et al. [33]), aluminum alloy to silver (e.g. Ying et al.[62]), as well as aluminum metal matrix composites (e.g. Fernandez et al. [16], Prado et al. [45-46] and Storjohann et al. [56]), etc.

\subsection{Problem Statement}

Although extensive work with FSW has been done on many materials, the material flow pattern in a friction stir weld is not yet fully understood. As the welding tool translates along the joint line, the shoulder and pin rotate and make a mixing of the workpiece material around the welding tool. The mixing and forging force create the joints between two workpieces. Welding parameters (translational and rotational velocities, forging force), tool geometry especially for the pin shape, and the characteristics of the material being joined will have an effect on the flow behavior. Therefore, being able to simulate the material flow pattern in a friction stir weld will help design the appropriate welding tool and optimize the welding parameters to produce a high-quality weld.

Investigation of material flow is performed either by experimentation or computer modeling. Some experiments (Colligan [9], Dickerson et al. [11], Guerra et al. [24], London et al. [39], Reyolds [47] and T.U. Seidel et al. [52-53]) have been conducted to track material flow in a friction stir weld. The method employed in such experiments is based on the use of a marker material which is different from the workpiece material being welded and redistributed during welding. The markers are placed in strategic locations within the weld in order to document the material flow associated with that particular region. After the weld is completed, the marker flow is revealed by a serial 
sectioning technique. The workpieces are cut into several successive slices and for each cut the section is polished, etched and digitalized. It is obvious that such an experiment is a direct method to understand the material flow pattern during FSW. To perform such an experiment, however, extensive background work with different marker materials is needed in order to select an appropriate marker material used in the subsequent experiments. Additionally, different types of welding tools need to be prepared in such experiments corresponding to the workpiece material variation. So, it is very costly and time-consuming to conduct these types of experiments. Moreover, only a couple of points in the region of interest are available for experimental measurement and acquired data for experimental analysis are very limited.

Compared to experimentation, modeling FSW can be very useful because it can provide a convenient and straightforward way to vary material properties over a wide range and the analysis also provides direct information of material flow, avoiding or minimizing trial and error. While the number of materials that can be welded to good quality by FSW are increasing, fully comprehensive modeling of the process will take its place as a major development tool alongside experimental tests, reducing the amount of time and resources required for full experimental trials, enabling further improvement of the process and allowing friction stir welding to reach its full potential.

The challenging issue in modeling FSW is the finite element mesh motion problem as the workpiece material flows around the welding tool. The method to handle large deformation and material flow determines if the modeling of FSW is successful. In this research, an effective mesh motion scheme particular for the problem of FSW has 
been developed and by the use of this method, the FSW process is simulated and the material flow pattern in a friction stir weld is described.

\subsection{Objectives of the Work}

This work has the following two aims:

- To develop a mesh motion scheme for simulating the large deformations of the workpieces in the FSW process as an alternate process to adaptive meshing schemes.

- To assess the material flow behavior of the rigid-elastoplastic problem of FSW using the moving mesh approach in order to establish approach effectiveness.

To achieve these objectives, the FSW process for the butt welds of same and dissimilar aluminum alloys is chosen for the numerical model. Process simulations are based on solid mechanics finite element formulations and carried out using the generalpurpose software LS-DYNA (Theoretical Manual [26] and User's Manual [40]).

The ultimate goal of this work is to apply such models to help design the welding tool and optimize the process parameters for FSW of different alloys. 


\subsection{Structure of the Dissertation}

The aspects are presented in 7 chapters as follows:

Papers that documented the material flow during FSW are reviewed in Chapter 2. These papers may be divided into two categories: experimental work and numerical simulation. The method and results in each work are briefly described. In addition, in this chapter, three kinds of finite element formulations and their applications to non-linear solid mechanics are reviewed.

Chapter 3 addresses numerical techniques behind the finite element program applied to FSW simulation. The emphasis is to derive Arbitrary Lagrangian-Eulerian (ALE) formulations. The chapter begins with a discussion of the material and mesh motions in an ALE framework. Then the conservation and constitutive equations for an ALE description are derived. The relevant finite element matrix equations are computed based on the ALE strong and weak forms. Finally the solution procedure based on the operator split technique is shown.

Chapter 4 presents FSW simulation using Lagrangian and ALE formulations. The simulation will show that the FSW process is difficult to handle with Lagrangian formulations. However it does not present any difficulty in ALE formulations.

Chapter 5 shows a mesh motion scheme particularly for the ALE modeling of FSW. By the use of this scheme, four cases documenting the material flow in the friction stir butt weld of aluminum alloys are studied. As the first case, the material flow in the friction stir weld of the same aluminum alloy is modeled to demonstrate its capabilities and potential in simulating FSW. The method is then applied to simulating the FSW process with different material models. Furthermore, the material flow in the friction stir 
weld of the dissimilar aluminum alloys is investigated using the same method. The last case addresses friction effect on the material flow pattern in the friction stir weld of the same aluminum alloy.

Chapter 6 discusses the FSW simulation results. The finite element predictions of the material flow pattern during FSW are compared with the published experimental results.

Finally, the work is summarized, contribution to the state of the art in FSW simulation is addressed, conclusions are drawn and areas of future research concerning the FSW simulation are suggested in Chapter 7. 


\section{CHAPTER 2 LITERATURE REVIEW}

In this chapter, two topics are addressed. To begin with, the previous experimental works and numerical studies of FSW with respect to material flow are reviewed. Fully understanding the material flow and mixing during FSW is very important for design of the welding tool and control of process parameters to produce high-quality welds. Second, three kinds of finite element formulations, namely Lagrangian, Eulerian, and Arbitrary Lagrangian-Eulerian (ALE) and their applications in non-linear solid mechanics are discussed. The discussions are focused on ALE formulations, which will be applied to the modeling of FSW.

\subsection{Experimental Works and Numerical Studies of the Material Flow during FSW}

The previous experimental works of the material flow during FSW were published in the papers (Colligan [9], Dickerson et al. [11], Guerra et al. [24], London et al. [39], Reyolds [47] and Seidel et al. [52-53]). The experimental technique to track material flow in a friction stir weld is based on the use of a marker material, which is different from the workpiece material being welded and redistributed during welding. There are three main requirements of a microstructural marker (London et al. [39]): it should be easily distinguished from the surrounding alloy after welding; it should readily flow with the alloy being welded; and it should be of a size scale that will not adversely affect the normal FSW material flow behavior. Different marker materials have been

used by researchers to track the material flow during FSW. Colligan [9] studied the 
material flow in the welds of 6061 and 7075 aluminum alloys using two techniques: steel shot tracers and sudden-stop action of the welding tool. The experimental results showed that material was stirred only in the upper portion of the weld and that in the rest of the weld, material was simply extruded around the pin. Dickerson et al. [11] used thin copper strip to trace the material movement of 2024-T3 aluminum alloy and analyzed the influence of marker material on material flow within the welds. Guerra et al. [24] used a copper foil along the contact surface of the two plates made of the 6061 and 2195 aluminum alloys and stopped the tool in place during FSW to make clear the material flow pattern. The results showed that material was wiped from the advancing side first, and then extruded on the retreating side. London et al. [39] put the Al-SiC and Al-W composite markers into 7075 aluminum alloy and investigated the material flow on the regions at the mid-plane and on the advancing side of the weld. Reyolds [47] and Seidel et al. [52-53] described the material flow of AA2195-T8, an aluminum-lithium alloy, in several friction stir welds using AA5454-H32 markers. Their experiments also showed the changes in material flow due to different welding parameters and tool geometries.

Compared to experimentation, computer simulation could be valuable on characterizing and optimizing of the FSW process since it is inexpensive, convenient and time-saving. Many numerical studies of FSW have been reported in the literature. Most efforts were made on temperature analysis and thermomechanical modeling (e.g. Chao et al. [5-6], Chen et al. [7], Dickerson et al. [12], Dong et al. [15], Gould et al. [23], Schmidt et al. [50-51], Song et al. [55], Ulysse [60]). However, a greater challenge modeling is to model the complex material flow in a friction stir weld. Modeling on this subject can fall into two categories: solid mechanics based models and fluid mechanics based models. 
Solid mechanics based modeling efforts were reported in the papers (Askari et al. [1], Deng et al. [10], Goetz et al. [22], Xu et al. [61]). Deng et al. [10] and Xu et al. [61] developed two-dimensional finite element models using general-purpose code ABAQUS to predict the material flow during FSW. Arbitrary Lagrangian-Eulerian (ALE) finite element formulations were used to deal with the large deformation of the workpiece material. Two types of pin/workpiece interfaces, namely the slipping interface and the friction contact were analyzed in the models. It was concluded that the workpiece material directly in front of the rotating pin moved behind the pin only from the retreating side. This result was compared qualitatively well with the experimental observations and was consistent with results from the research in this dissertation. Askari et al. [1] studied three-dimensional material flow in the friction stir weld using Eulerian and finite difference methods, which were implemented into the CTH code: a three-dimensional hydro-code for shock wave processing. The strains, strain rates and thermal histories were predicted in the work. Modeling results were compared with experimental observations and they agreed with each other. Goetz et al. [22] used a two-dimensional finite element code, DEFORM, which was originally developed for metal forging, to simulate material flow in the weld and the initial penetration of the tool into the workpiece.

The second way to model material flow in a friction stir weld is based on fluid mechanics. The modeling method is to treat the workpiece material as a viscous fluid and the viscosity is dependent on strain rate and temperature. Bendzsak et al. [3] presented preliminary results from three-dimensional heat and material flow simulations in which viscous dissipation was the heat source. Frictional heating between the rotating tool and 
the workpiece was not considered. Colegrove et al. [8] developed two-dimensional models to simulate metal flow around the profiled tool using Computational Fluid Dynamics (CFD) code. The slip and stick boundary conditions between the workpiece and tool were considered in the models. Velocity vectors, streamline, torque and force for various welding parameters were generated, and validated against experiments.

It is noted that the above numerical attempts on the material flow prediction have some deficiencies in their work. Firstly, FSW is a three-dimensional asymmetric process, so two-dimensional models couldn't fully capture material flow taking place in such a process. Secondly, FSW is a solid-state process in which the workpiece material is not melted. The fluid mechanics based models could not accurately account for such a solidstate process. That is because those models neglected any effect from elastic strain and work hardening of material.

The difficulties in modeling material flow during FSW are mainly related to the complexity of the actual process. FSW involves large deformation of the workpiece material caused by the rotation of the tool; frictional contact between the workpieces and rotating tool, temperature increase in the weld due to frictional heat and large plastic work but it is below the melting point of the workpiece material. Considering that all those factors are combined into a finite element model, it is quite challenging to do it. Apart from the complexity of the actual process, there are some aspects that make the modeling of material flow particularly difficult. As the welding tool proceeds, the workpiece material deforms highly, causing the severe distortions of the finite element mesh. The finite element results then deteriorate and even the computation terminates. On the other hand, the difficulties in predicting material flow behavior are due to the lack of 
detailed material characteristic information such as the frictional coefficient and other thermal physical properties at elevated temperatures.

\subsection{Lagrangian, Eulerian, ALE Formulations and Their Applications in Nonlinear Solid Mechanics}

\subsubsection{Lagrangian and Eulerian Formulations}

When non-linear solid mechanics processes are simulated using finite element methods, two kinds of formulations are commonly applied to delineating distortions of a continuum, namely Lagrangian and Eulerian. In nature, both of them are used to determine the relationship between the deformed continuum and the mesh of computing zones. But they each have advantages and disadvantages and apply to solving different problems.

In a Lagrangian description, the nodes of finite element mesh are attached to the material points and the mesh deforms with the material, as illustrated in Fig.2-1. Since the nodes coincident with the material points in the Lagrangian mesh, boundary nodes remain on the boundary throughout the evolution of the problem. This simplifies the imposition of boundary conditions in Lagrangian meshes. The Lagrangian description is most popular in solid mechanics. Its attractiveness is that it can easily handle complicated boundaries and accurately treat history dependent materials. However, this approach is not suitable for large deformation problems since the material deforms largely and the mesh is also severely deformed, causing poor results and even computational termination.

On the other hand, in an Eulerian description, the nodes of finite element mesh are

fixed in space and the material flows through the mesh, as shown in Fig.2-1. The material properties are calculated at fixed spatial locations as the material flows through the mesh. 
Boundary nodes do not remain coincident with the boundary. Therefore, boundary conditions must be imposed at points which are not nodes. This approach is more suitable for fluid mechanics problems where often no history dependent materials are used. This approach has also been used to model large deformation of solids, mostly in metal forming analyses. Due to the fixed nature of the approach, it is most suitable for cases where there are minimal free boundaries, i.e. where the boundaries of the material region are known a priori. Examples of these cases are closed-die forging and extrusion problems. The Eulerian approach can deal with the strong distortions of mesh. However, it is difficult for this approach to treat history dependent materials and the greatest disadvantage is that a fine mesh is required to capture the material response, making this method computationally expensive. This is particularly true for problems that contain regions where the structural response is desired and the strains are relatively small. 

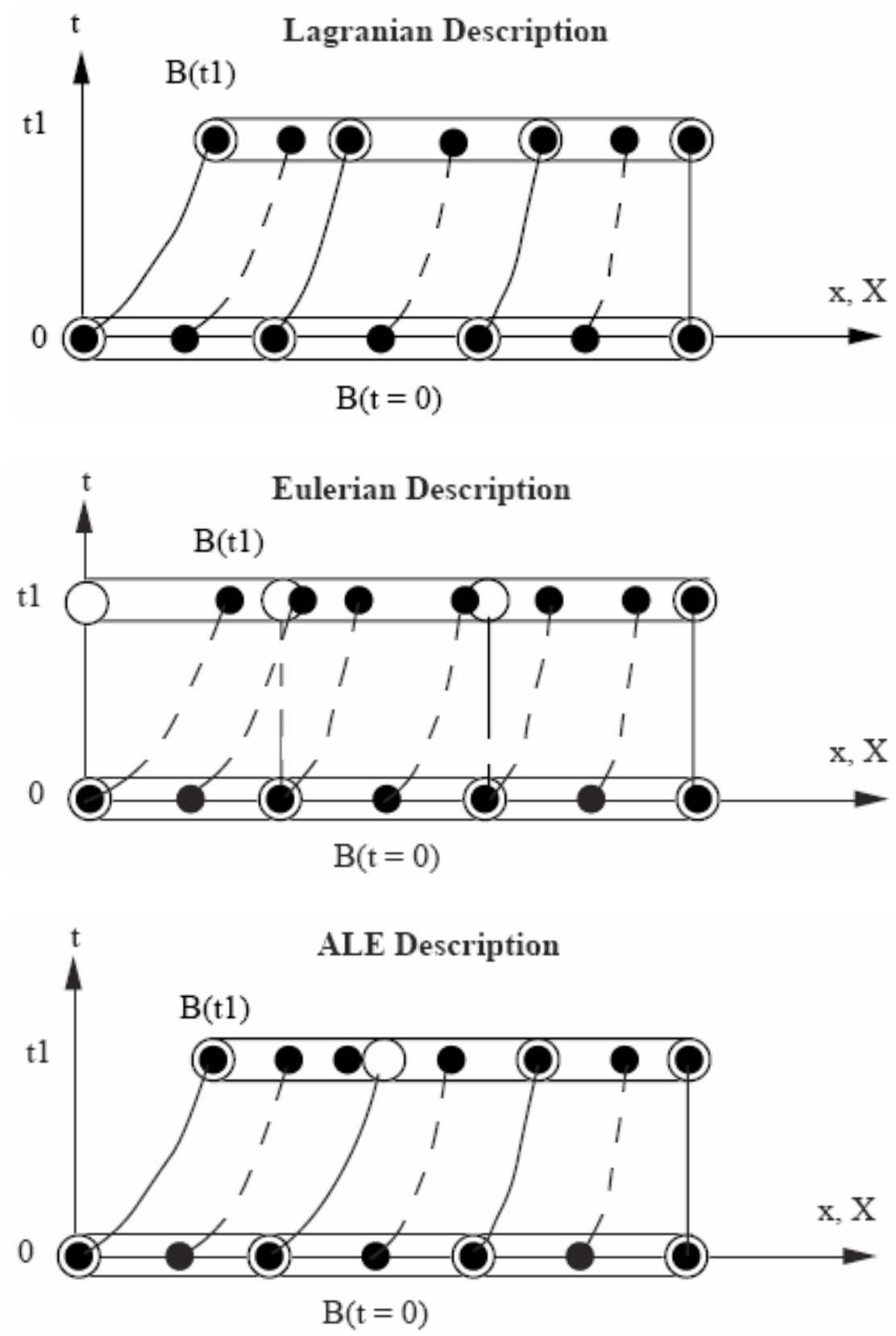

Figure 2-1 One Dimensional Example of Lagrangian, Eulerian and ALE Mesh and Material Point Motion (Belytschko et al. [2]) 


\subsubsection{Arbitrary Lagrangian-Eulerian (ALE) Formulations}

It is evident that the Lagrangian and Eulerian approaches can not be used to simulate the deformation process involving both large deformation and free flow of material, and a better formulation must include aspects of both methods. Therefore, the third type formulation, namely Arbitrary Lagrangian Eulerian (ALE), which will be implemented to simulate the material flow during FSW, has been developed in an attempt to combine the advantages of Lagrangian and Eulerian in a single analysis, while minimizing their disadvantages as much as possible.

In an ALE description, the nodes of finite element mesh are neither attached to the material points nor fixed in space. Instead, they may have an independent and arbitrary motion prescribed by the users, as shown in Fig.2-1. Usually the nodes on the boundaries are moved to remain on the boundaries, while the interior nodes are moved to minimize mesh distortion. The ALE approach is a very effective alternative for simulating large deformation problems. The greatest advantage is that it allows smoothing of a distorted mesh without performing a complete remesh. This smoothing allows the free surface of the material to be followed automatically without encountering the distortional errors of the Lagrangian approach. The main difficulty of the ALE method is the path dependent behavior of the plastic flow being modeled. Due to the path dependence, the relative motion between the mesh and the material must be accounted for in the material constitutive equations.

ALE formulations were originally developed for fluid dynamics and fluidstructure interaction problems (e.g. Donea [13]), and were later extended to solid mechanics applications (e.g. Donea [14]). Particular efforts were made to simulate non- 
linear solid mechanics problems such as impact and metal forming (e.g. Huerta et al. [30] and Huétink et al. [31]), crack propagation (e.g. Koh et al. [34] and Movahhedy et al. [41]), and cutting processes (e.g. Movahhedy et al.[42] and Olovsson et al. [43] ), etc, which involve large deformation of material.

The fundamental description in ALE formulations is to determine the relationship between the deformed continuum and computational mesh, which is also called kinematic description. Under this description, ALE conservation equations, which govern the motion of the continuum, and ALE constitutive equations, which reflect the behavior of the continuum, are derived. There are two kinds of ALE constitutive equations for two types of material models, namely hypoelastic-plasticity (Belytschko et al. [2]) and hyperelastic-plasticity (Rodríguez-Ferran et al. [49]). Hypoelastic-plastic models are based on the additive decomposition of the rate of deformation into elastic and plastic parts. In these models, constitutive equations are expressed as the relation between the rate of stress and the rate of deformation. But hyperelastic-plastic models are based on a multiplicative decomposition of the deformation gradient into elastic and plastic parts. Their constitutive equations are not described in terms of a rate form as written in hypoelastic-plastic models.

In the ALE formulations for non-linear solid mechanics, both conservation and constitutive equations contain convective terms accounting for the relative motion between the material and mesh. Those terms increase the difficulty in solving ALE equations compared to the traditional Lagrangian computation. Two methods are used to solve them: fully coupled and operator split. The fully coupled approach solves the equations as they are written and no further simplifications are made on these equations. 
S. Ghosh et al. [21] and W.K. Liu et al. [38] employed the fully coupled method to solve the ALE equations.

The operator split approach is relatively simple. To begin with, a Lagrangian time step is performed. During this step, the mesh moves with the material and the solutions to a standard Lagrangian problem are obtained. The convective effect is ignored in this step. Sequentially an advection step is carried out and has to consider the convective effect. This step is performed in one increment and consists of two sub-steps, i.e. smoothing step and remap step. First, the distorted mesh caused by the Lagrangian step is smoothed out by the use of the mesh smoothing algorithms. The algorithms for moving the mesh relative to the material control the range of the problems that can be solved by ALE formulations. In LS-DYNA code, four different mesh smoothing algorithm options can be chosen, i.e. simple average, volume-weighted, equipotential and equilibrium. For the simple average method, the coordinates of a node are the simple average of the coordinates of its surrounding nodes. Volume-weighted method uses a volume-weighted average of the coordinates of the centroids of the elements surrounding a node. Equipotential zoning is a method of making a structured mesh for finite difference or finite element calculations by using the solutions of Laplace equations as the mesh lines. The same method can be used to smooth selected points in an unstructured 3-D mesh provided that it is at least locally structured. This method will be discussed in detail in Chapter 3. For Equilibrium method, artificial springs are attached to each ALE element node. The springs are used to adjust the position of each node from the equilibrium solution. This approach can overcome possible calculation instabilities found in the other smoothing methods. After the new mesh is created, the remap step maps the solution 
from a distorted Lagrangian mesh onto the new mesh. The underlying assumptions of the remap step are (1) the topology of the mesh is fixed (that is, the element nodal connectivity remains unchanged), and (2) the mesh motion during a step is less than the characteristic lengths of the surrounding elements. The algorithms for performing the remap step are taken from the computational fluid dynamics community and are referred to as "advection" algorithms. Benson [4] presented a simple ALE formulation in which the operator split approach was used to separate Lagrangian and Eulerian processes. Compared to the fully coupled approach, split operator breaks very complicated equations into several simpler equations which are solved easily. Algorithms that rely on an operator split are therefore usually cheaper and more robust than algorithms that try to solve the fully coupled problem.

Contact treatment in ALE formulations is another important issue that should be discussed here. It is different from that in traditional Lagrangian formulations. Since ALE formulations allow the mesh motion independent of the material motion, a contact algorithm can be avoided in some situations. Numerical illustrations of this particular case can be found in the literature (Gadala et al. [18-19]). However, in more general situations, a contact algorithm can not be avoided. That is because the velocity difference between the mesh nodes and the material points has to be considered for the historydependent contact variables. For example, in the contact model developed by Ghosh [20], ALE nodes on one of the contacting surfaces were constrained to follow the motion of the Lagrangian nodes on the other surface. In another case, Haber et al. [25] updated the mesh in order to make the contacting node pairs and element edges not to be changed in the deformed configuration, thus ensuring a precise satisfaction of geometric 
compatibility and allowing a consistent transfer of contact stresses between the two bodies.

Here, three representative numerical simulations are shown to present the applicability of ALE formulations. First example is a cylindrical bar impacts a rigid frictionless wall at an initial velocity (Huerta et al. [30]). An axisymmetric model was developed and the material is assumed elastoplastic. In Fig.2-2, a comparison of the calculated deformed mesh between Lagrangian and ALE formulations at different time instances is presented. One can see the difference between the Lagrangian and ALE calculations for the radius and the final length of the bar are small. But important differences are observed in the time step $\Delta t$ of the dynamic computation, as shown in Fig.2-3. The ALE calculation can keep larger and more stable time steps than the Lagrangian calculation. That means the ALE calculation is faster than the Lagrangian for the required simulation. Fig.2-4 shows the final yield stresses calculated using Lagrangian and ALE formulations. Both results are similar. 


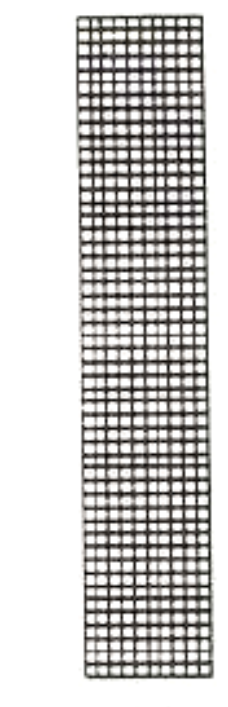

$$
\text { Initial }(t=0) \quad t=10 \mu \mathrm{s}
$$

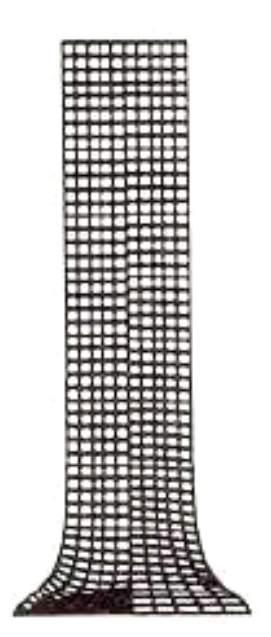

$t=20$

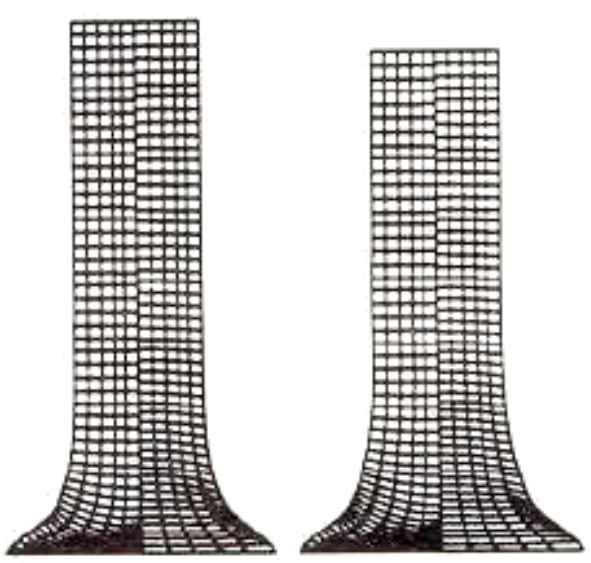

$t=40$

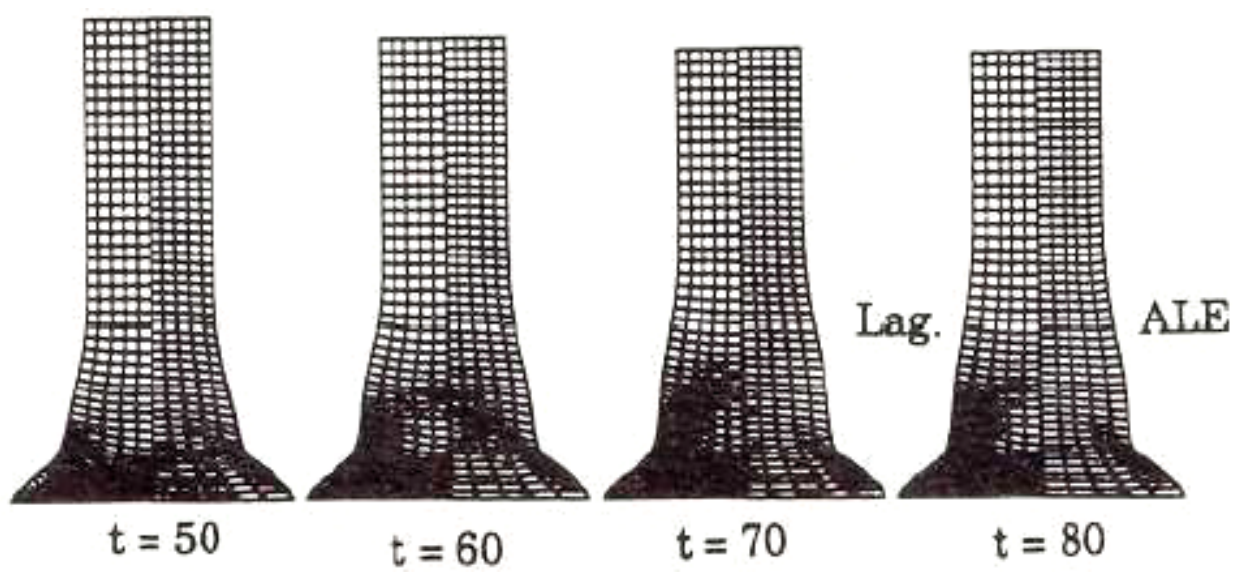

Figure 2-2 Comparison of Spatial Lagrangian Mesh (left) and ALE Mesh (Right) at Different Time Instances (Huerta et al. [30]) 


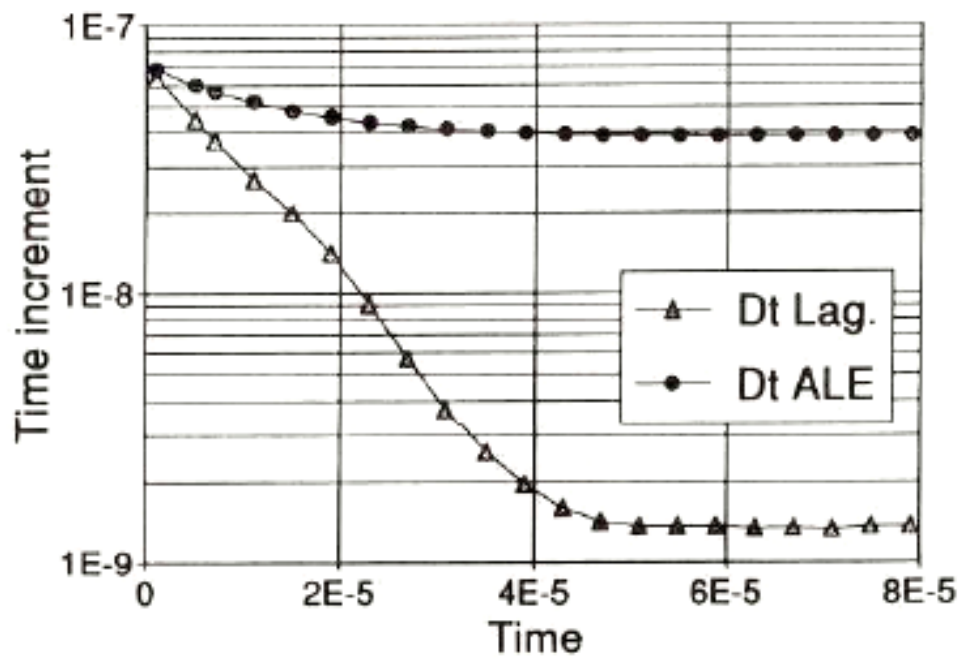

Figure 2-3 Variation of Time-increment, $\Delta t$, with Time (during the Deformation Process) (Huerta et al. [30])

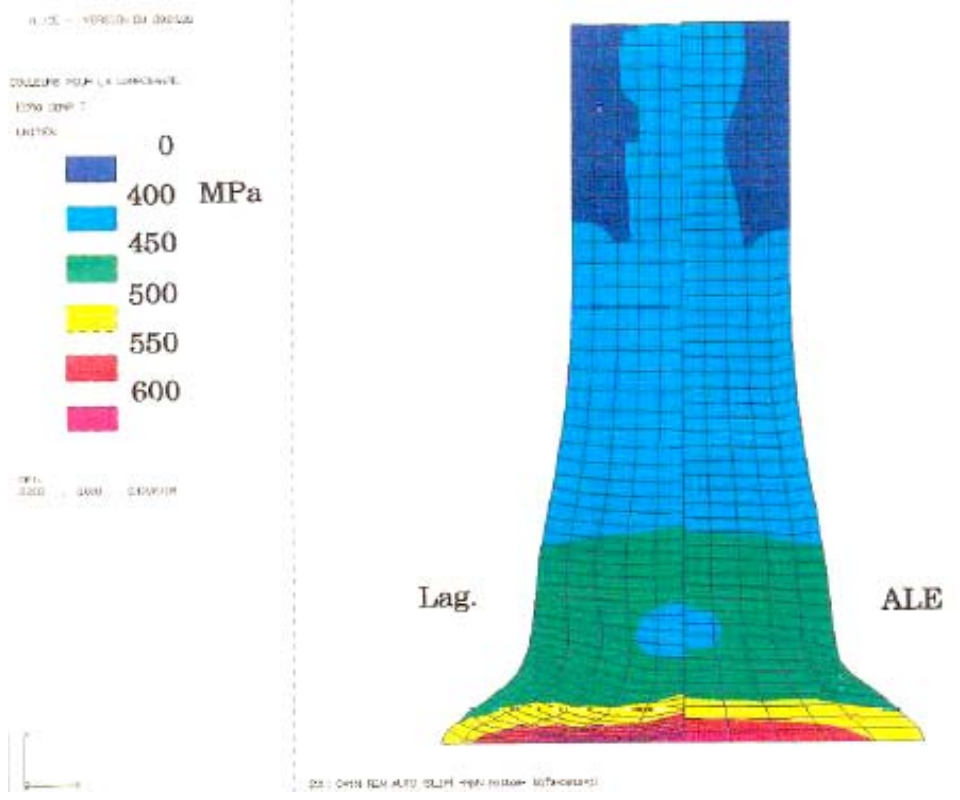

Figure 2-4 Comparison between Final Yield Stress Distributions Calculated Using Lagrangian and ALE Formulations (Huerta et al. [30]) 
A second example is the necking of a cylindrical bar (Linder [37]), which is pulled with an initial velocity in the vertical direction, as illustrated in Fig.2-5(left). The bar material is assumed as a von Mises model with isotropic hardening. Due to symmetry only the quarter of the bar is modeled using Lagrangian and ALE formulations respectively. Fig.2-5(right) shows the discretization of half the bar.

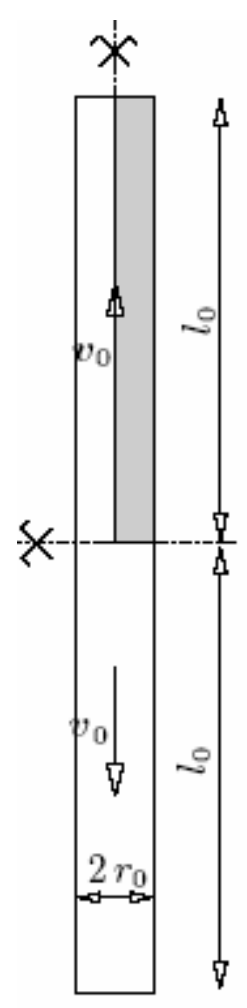

Problem Definition

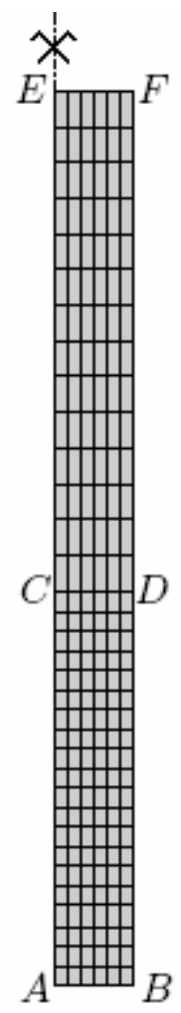

Disretization

Figure 2-5 Pulling a Cylindrical Bar and Finite Element Discretization (Linder [37])

Fig.2-6(a) and (b) show the spatial Lagrangian and ALE mesh at different time instances. Since the mesh nodes move with the material points in the Lagrangian computation, one could see the distorted Lagrangian elements in the necking zone. However, the spatial ALE mesh is well distributed in the ALE computation. That is because the distorted mesh caused in the Lagrangian step is smoothed out by the 
following smoothing strategy. The vertical positions of the nodes in the lower domain $\mathrm{ABCD}$ are fixed, and in horizontal direction the elements are enforced to be equidistantly distributed. In addition, in the upper domain CDEF the elements are enforced to be equidistantly distributed in the vertical as well as the horizontal directions.

Fig.2-7 shows the different distributions of the von Mises stress in the necking zone at $t=20 \mu \mathrm{s}$ for the Lagrangian and ALE calculations, respectively. The ALE calculation shows the accurate results since the von Mises stress in the necking zone should be constant in horizontal direction. However, Due to the distorted mesh the Lagrangian calculation shows the inaccurate results.

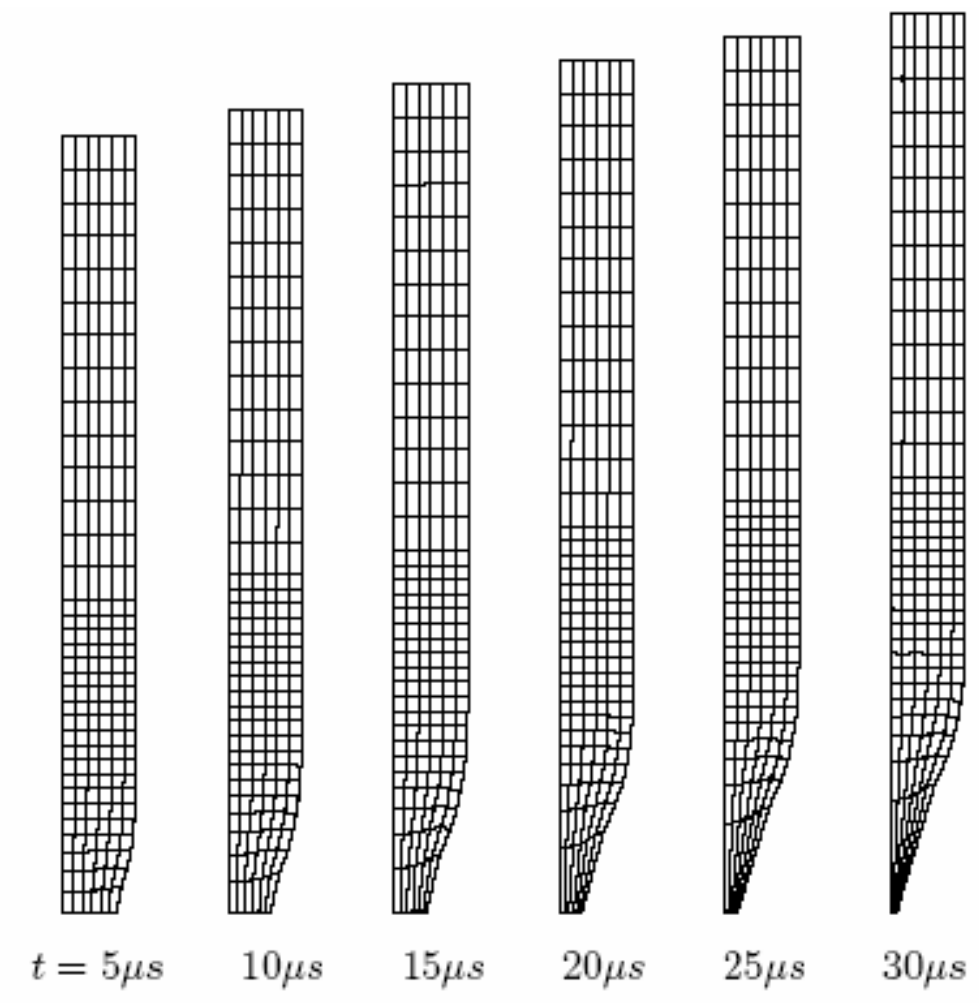

(a) 

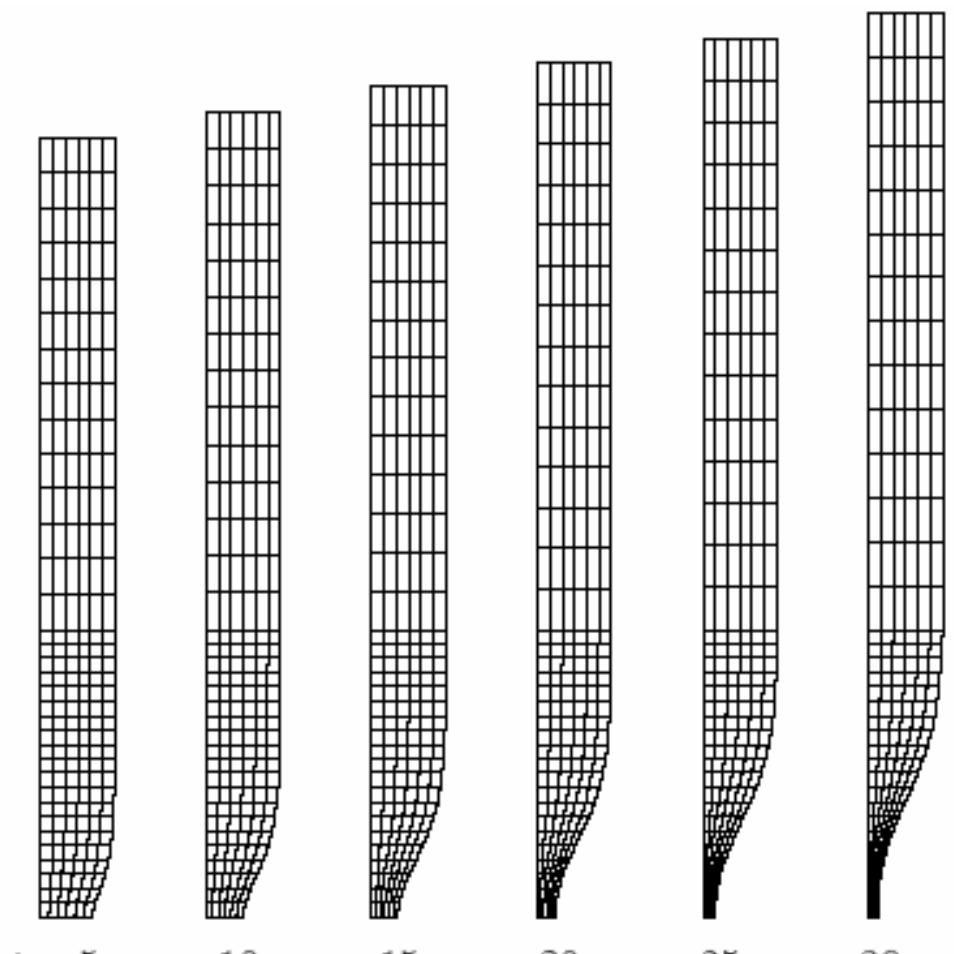

$t=5 \mu \mathrm{s}$

$10 \mu s$

$15 \mu s$

$20 \mu s$

$25 \mu s$

$30 \mu s$

(b)

Figure 2-6 (a) Deformed Lagrangian Mesh and (b) Well Distributed ALE Mesh at Different Time Instants (Linder [37])

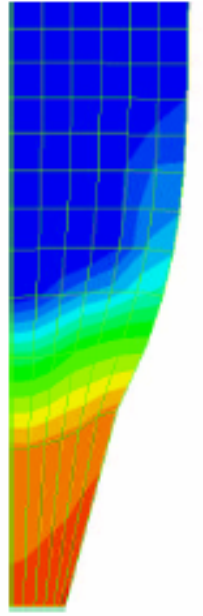

Lagrange

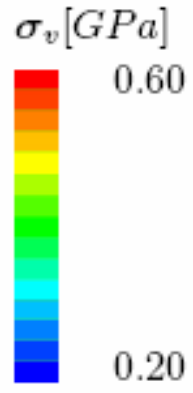

0.20

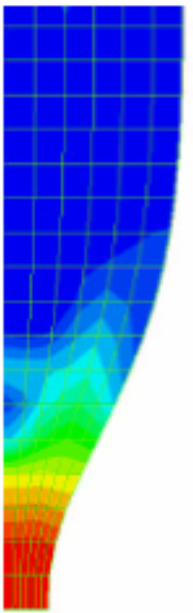

ALE

Figure 2-7 Von Mises Stress Distribution in the Necking Zone at $t=20 \mu$ for Lagrangian and ALE Computation (Linder [37]) 
The last example is a coining process (Linder [37]), where the body is deformed by a punch with a prescribed velocity $v$ in the vertical direction. Due to symmetry, only half of the domain is simulated, as shown in Fig.2-8. The body is assumed as a von Mises model with isotropic hardening and punch is modeled as rigid. Fig.2-9 shows the Lagrangian and ALE mesh at different height reductions. Due to the large deformation of the finite elements the Lagrangian computation breaks off at a height reduction of about $30 \%$. However, ALE formulations can cope with these difficulties by the use of the following smoothing strategy. The elements in the vertical direction are equidistantly distributed and parabolic profiles of horizontal mesh displacements are prescribed. 


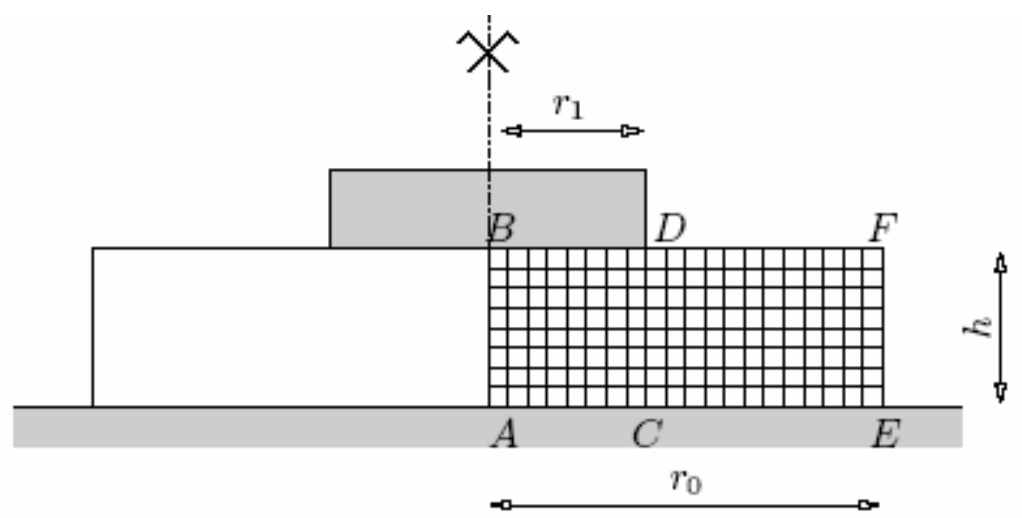

Figure 2-8 Coining Test and Finite Element Discretization (Linder [37])

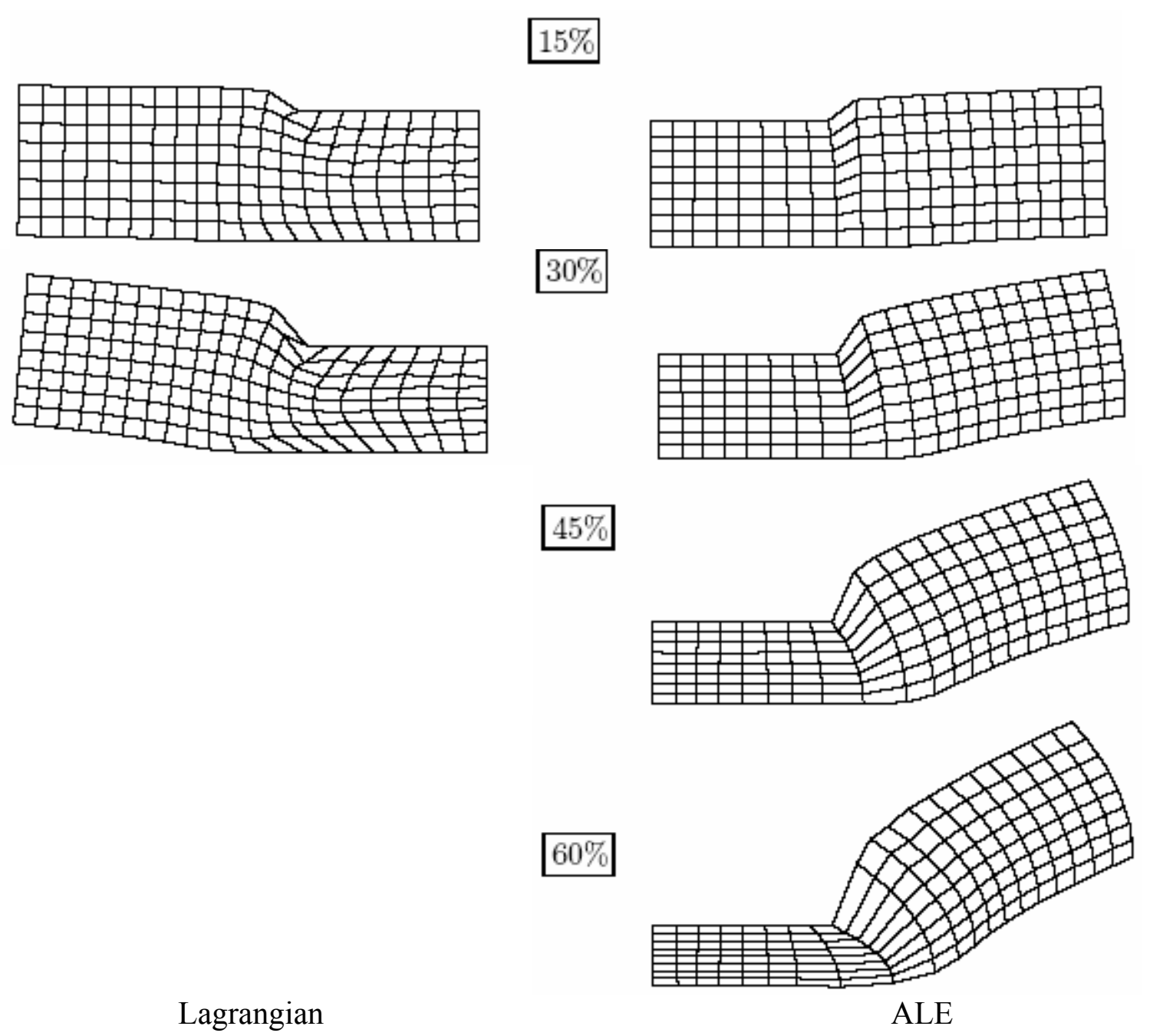

Figure 2-9 Spatial Lagrangian and ALE Mesh at Different Reduction (Linder [37]) 


\subsection{Literature Review Summary}

From the literature review, first it is noted that the studies on the material flow pattern during FSW are very limited, especially for numerical modeling. In addition, the existing the fluid- and solid- mechanics based models have some deficiencies. Second, it is shown that ALE formulations are the most effective methods for simulating large deformation problems in non-linear solid mechanics compared to Lagrangian and Eulerian formulations. Therefore, in this research, ALE formulations are chosen to simulate the FSW process which involves large deformation of the workpiece material. The algorithms have been implemented into the general-purpose code LS-DYNA. In the next chapter, the ALE formulations applied to FSW simulation will be discussed in detail. 


\section{CHAPTER 3 ARBITRARY LAGRANGIAN-EULAERIAN (ALE) FORMUALTIONS APPLIED TO THE SIMULATION}

To simulate FSW by the use of ALE formulations, the ALE form of the conservation equations for mass, momentum and energy as well as constitutive equations is needed. These equations are solved to find the spatial distributions of displacement, strain, stress and temperature over the workpiece material at any time during FSW.

In this chapter, ALE formulations meant for solving the problem of FSW are presented. To begin with, the ALE kinematics is described, i.e. the relationship between the material and mesh motions. Then the fundamental ALE equation is derived, which determines the relationship between the material time derivative and referential time derivative. Second, based on the fundamental ALE equation, the ALE form of conservation and constitutive equations is established. Thirdly, the coupled finite element matrix equations for the conservations of mass and momentum as well as constitutive equations are derived. ALL these equations include the convective term that accounts for the difference between the material and mesh velocities. It is this additional term in each equation that makes solving ALE equations much more difficult numerically than Lagrangian equations. The operator split method is applied to solving ALE equations in two steps: a Lagrangian step and an advection step. Therefore, finally, the numerical techniques associated with these two steps are discussed. The discussions in this chapter follow the presentations in Belytschko et al. [2], Benson [4], Hallquist [26], and Linder [37]. 


\subsection{Fundamentals}

To describe the motion of a continuum using classical Lagrangian formulations, a one-to-one mapping between the initial position $X$ of a material point and its final spatial position $x$ is needed, as illustrated in Fig.3-1. All the material points $\mathbf{X}$ form a material domain and all the spatial points $\mathbf{x}$ compose a spatial domain. The mapping function $\varphi(\mathbf{X}$, $t$ ) is defined to map the material domain onto the spatial domain at the time, $t$.

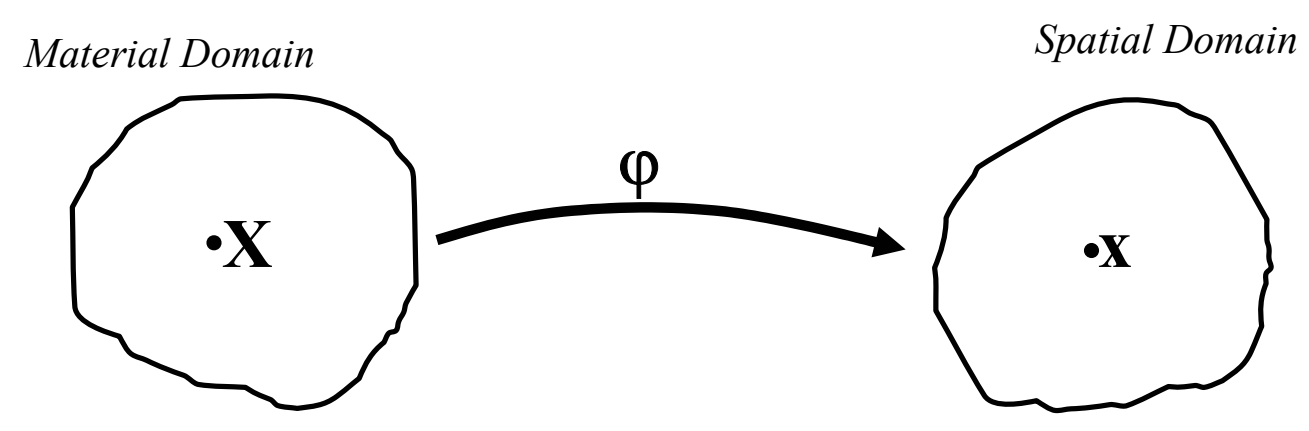

Figure 3-1 Lagrangian Kinematic Description

In the ALE description, since the mesh motion is arbitrary and is independent of the material motion, an additional domain except the material and spatial domains is introduced to identify the mesh points, which is called the referential domain or ALE domain, as illustrated in Fig.3-2. The coordinates of any point in this domain are denoted as $\chi$. Now the mappings relating the referential domain with the material and spatial domains need to be given. The functions $\Psi(\chi, t)$ and $\Phi(\chi, t)$ are defined to map the referential domain onto the material and spatial domain at time $t$, respectively. It is noted that even though three functions are required to map three domains with each other, they are not independent but satisfy Equation $\varphi(\mathbf{X}, t)=\boldsymbol{\Phi}(\chi, t) \cdot \boldsymbol{\Psi}^{-1}(\mathbf{X}, t)$. In the following, we 
present the motion of the material and the mesh using these functions in the ALE description.

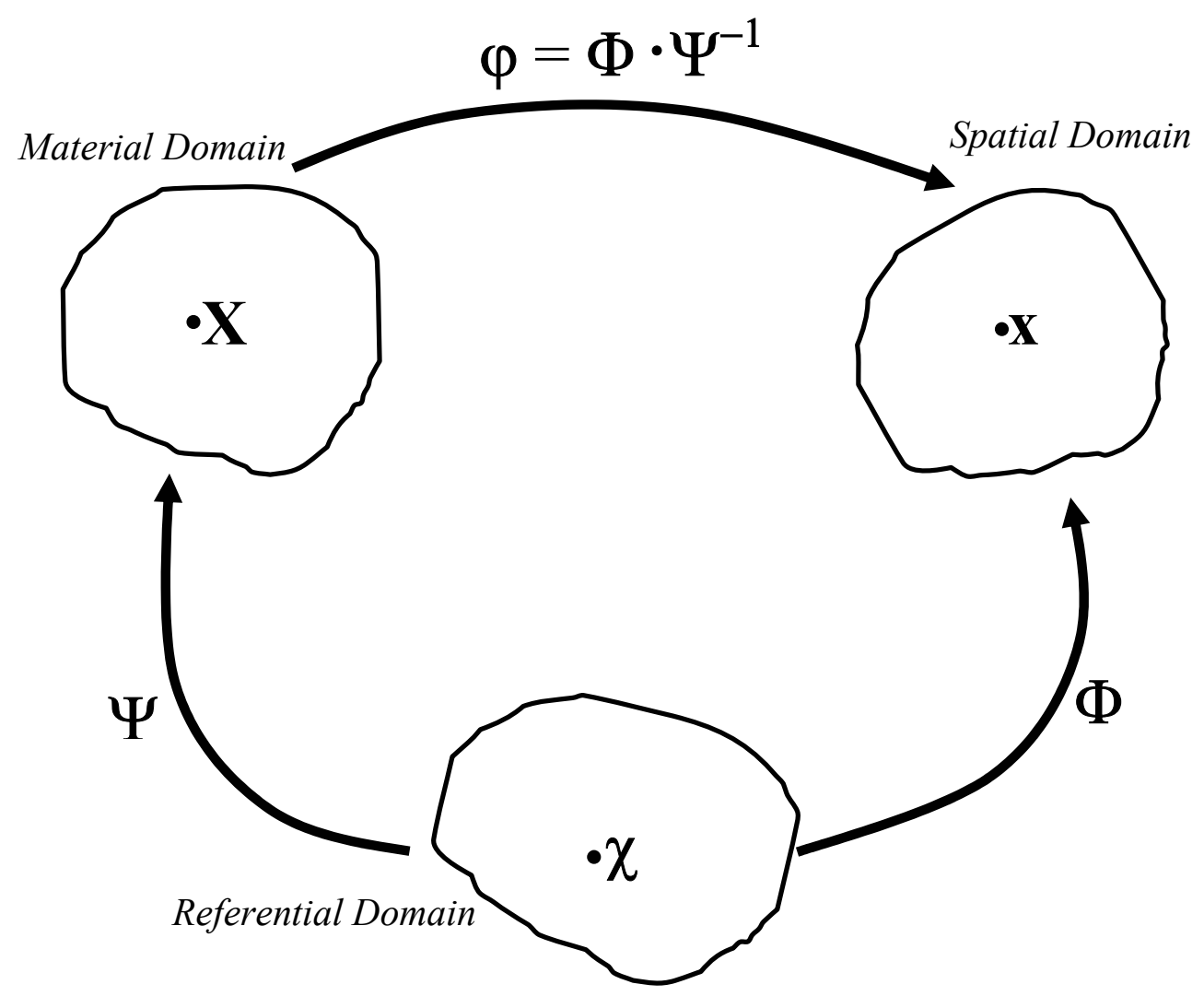

Figure 3-2 ALE Kinematic Description

The mapping $\varphi$ from the material domain to the spatial domain describes the motion of the material points in the spatial domain. So, the motion of the material is expressed by

$$
\mathbf{x}=\varphi(\mathbf{X}, t)
$$

The mapping $\Phi$ relating the referential domain with the spatial domain can be understood as the motion of the mesh in the spatial domain which is given by

$$
\mathbf{x}=\boldsymbol{\Phi}(\chi, t)
$$


Although the mapping $\varphi(\mathbf{X}, t)$ is different from mapping $\Phi(\chi, t)$, the spatial coordinates are the same. So, the following equation is obtained

$$
\mathbf{x}=\varphi(\mathbf{X}, t)=\mathbf{\Phi}(\chi, t)
$$

With the basic definitions of the material and mesh motion it is now possible to define displacement, velocity and acceleration of a typical material point $X$ and a mesh point $\chi$. Material displacement, $\mathbf{u}$, velocity, $\mathbf{v}$, and acceleration, $\mathbf{a}$, are:

$$
\begin{aligned}
& \mathbf{u}(\mathbf{X}, t)=\mathbf{x}-\mathbf{X}=\varphi(\mathbf{X}, t)-\mathbf{X} \\
& \mathbf{v}=\left.\frac{\partial \varphi(\mathbf{X}, t)}{\partial t}\right|_{\mathbf{X}}=\left.\frac{\partial \mathbf{x}}{\partial t}\right|_{\mathbf{X}} \\
& \mathbf{a}=\left.\frac{\partial \mathbf{v}}{\partial t}\right|_{\mathbf{x}}=\left.\frac{\partial^{2} \mathbf{x}}{\partial t^{2}}\right|_{\mathbf{x}}
\end{aligned}
$$

Mesh displacement $\hat{\mathbf{u}}$, velocity $\hat{\mathbf{v}}$, and acceleration $\hat{\mathbf{a}}$ are:

$$
\begin{aligned}
& \hat{\mathbf{u}}(\chi, t)=\mathbf{x}-\chi=\boldsymbol{\Phi}(\chi, t)-\chi \\
& \hat{\mathbf{v}}=\left.\frac{\partial \boldsymbol{\Phi}(\chi, t)}{\partial t}\right|_{\chi}=\left.\frac{\partial \mathbf{x}}{\partial t}\right|_{\chi} \\
& \hat{\mathbf{a}}=\left.\frac{\partial \hat{\mathbf{v}}}{\partial t}\right|_{\chi}=\left.\frac{\partial^{2} \mathbf{x}}{\partial t^{2}}\right|_{\chi}
\end{aligned}
$$

where $\left.\cdot\right|_{\mathbf{X}}$ and $\left.\cdot\right|_{\mathbf{x}}$ means holding the material coordinates $\mathbf{X}$ and the referential coordinates $\chi$ fixed.

Regarding function, $\Psi$, the following expression is useful,

$$
\chi=\Psi^{-1}(\mathbf{X}, t)
$$

so the material velocity in the referential domain can be expressed as

$$
\mathbf{w}=\left.\frac{\partial \chi}{\partial t}\right|_{\mathbf{x}}
$$


To obtain the relation between velocities, $\mathbf{v}, \hat{\mathbf{v}}$ and, $\mathbf{w}$, differentiating Equation (3-3) with respect to time and substituting Equation (3-4b), (3-5b) and (3-7) yield

$$
v_{j}=\left.\frac{\partial x_{j}}{\partial t}\right|_{\mathbf{x}}=\left.\frac{\partial x_{j}}{\partial t}\right|_{\chi}+\left.\frac{\partial x_{j}}{\partial \chi_{i}} \frac{\partial \chi_{i}}{\partial t}\right|_{\mathbf{x}}=\hat{v}_{j}+\frac{\partial x_{j}}{\partial \chi_{i}} w_{i}
$$

Rearrange Equation (3-8) as

$$
c_{j}=v_{j}-\hat{v}_{j}=\frac{\partial x_{j}}{\partial \chi_{i}} w_{i}
$$

where $c_{j}$ is the convective velocity, which is the relative velocity between the material and mesh.

Since the term of the material time derivative exists in conservation and constitutive equations which are required for solving the problem of FSW, the relationships between the material time derivative and the referential time derivative need to be established. Consider a scalar physical quantity $f$ which can be expressed as $f(\mathbf{X}, t), \bar{f}(\mathbf{x}, t), \hat{f}(\chi, t)$ in terms of the material, spatial and referential coordinates, respectively. Since these three different functions represent the same field, the following results are obtained

$$
f(\mathbf{X}, t)=\bar{f}(\mathbf{x}, t)=\hat{f}(\chi, t)
$$

Differentiating equation (3-10) with respect to time yields three different types of material time derivatives

$$
\begin{aligned}
& \frac{d f}{d t}=\left.\frac{\partial f}{\partial t}\right|_{\mathrm{x}} \text { (Lagrangian description) } \\
& \frac{d f}{d t}=\left.\frac{\partial \bar{f}}{\partial t}\right|_{\mathrm{x}}+\left.\frac{\partial \bar{f}}{\partial x_{i}} \cdot \frac{\partial x_{i}}{\partial t}\right|_{\mathrm{x}}=\frac{\partial \bar{f}}{\partial t}+\frac{\partial \bar{f}}{\partial x_{i}} v_{i} \text { (Eulerian description) }
\end{aligned}
$$




$$
\frac{d f}{d t}=\left.\frac{\partial \hat{f}}{\partial t}\right|_{\chi}+\left.\frac{\partial \hat{f}}{\partial \chi_{i}} \frac{\partial \chi_{i}}{\partial t}\right|_{\mathbf{x}}=\left.\frac{\partial \hat{f}}{\partial t}\right|_{\chi}+\frac{\partial \hat{f}}{\partial \chi_{i}} w_{i} \text { (ALE description) }
$$

Equation (3-11c) relates the material time derivative with the referential time derivative and the gradient of function $f$ in the referential domain. But, for convenience, spatial gradient of function $f$ is desired in calculation. Applying chain rule to Equation (311c) and using Equation (3-9) gives

$$
\begin{aligned}
\frac{d f}{d t} & =\left.\frac{\partial \hat{f}}{\partial t}\right|_{\chi}+\left.\frac{\partial \bar{f}}{\partial x_{j}} \frac{\partial x_{j}}{\partial \chi_{i}} \frac{\partial \chi_{i}}{\partial t}\right|_{\mathbf{X}} \\
& =\left.\frac{\partial \hat{f}}{\partial t}\right|_{\chi}+\frac{\partial \bar{f}}{\partial x_{j}} \frac{\partial x_{j}}{\partial \chi_{i}} w_{i} \\
& =\left.\frac{\partial \hat{f}}{\partial t}\right|_{\chi}+\frac{\partial \bar{f}}{\partial x_{j}} c_{j}
\end{aligned}
$$

Since $f, \bar{f}, \hat{f}$ represent the same physical quantity, Equation (3-12) can be written as

$$
\frac{d f}{d t}=\left.\frac{\partial f}{\partial t}\right|_{\chi}+\frac{\partial f}{\partial x_{j}} c_{j}
$$

Equation (3-13) gives the material (or total) time derivative of the physical quantity $f$, in terms of the partial time derivative with the referential coordinates fixed,

$\left.\frac{\partial \hat{f}}{\partial t}\right|_{\chi}$, and the spatial gradient, $\frac{\partial f}{\partial x_{j}}$. This equation is very important for the development of ALE conservation and constitutive equations since the material time derivative is inherent in those equations. It is observed that Equation (3-13) can be reduced to Equation (3-11a) when $\mathbf{c}=0$, which is the Lagrangian description; and Equation (3-11b) when $\mathbf{c}=\mathbf{v}$ which is the Eulerian description. 


\subsection{Governing Equations in ALE Formulations}

\subsubsection{Conservation Equations}

Three conservation laws relevant to the problem of FSW are considered here:

- Conservation of mass, which states that the mass of any material body is constant, since no material flows through the boundaries of a material body.

- Conservation of momentum, which states that the material time derivative of the momentum equals the net force. It is equivalent to Newton's second law.

- Conservation of energy, which states that the rate of change of the total energy (the internal plus kinetic energies) in the body is equal to the work done by the external forces and rate of work provided by heat flux and heat sources

Conservation laws are usually expressed as partial differential equations (PDEs). Three PDEs of mass, momentum and energy are expressed by Equations (3-14a), (3-14b) and (3-14c), respectively.

Mass conservation:

$$
\frac{\partial \rho}{\partial t}=-\rho \frac{\partial v_{j}}{\partial x_{j}}
$$

Momentum conservation:

$$
\rho \frac{\partial v_{i}}{\partial t}=\frac{\partial \sigma_{j i}}{\partial x_{j}}+\rho b_{i}
$$

Energy conservation:

$$
\rho \frac{\partial w^{\mathrm{int}}}{\partial t}=D_{j i} \sigma_{j i}-\frac{\partial q_{i}}{\partial x_{i}}+\rho s
$$


where $\rho$ is the mass density; $\sigma_{j i}$ is the Cauchy stress; $b_{i}$ is the body force per unit volume; $\rho w^{\text {int }}$ is the internal energy per unit volume; $D_{j i}$ is the rate of deformation ; $q_{i}$ is the heat flux per unit area; and $\rho$ s is the heat source per unit volume.

To obtain the ALE differential form of the conservation equations for mass momentum and energy, we just replace the material time derivative in the equations above by the ALE form of material time derivative Equation (3-13). For example, applying Equation (3-13) to Equation (3-14a), i.e. replacing $\frac{\partial \rho}{\partial t}$ with $\left(\left.\frac{\partial \rho}{\partial t}\right|_{\chi}+\frac{\partial \rho}{\partial x_{j}} c_{j}\right)$ gets the ALE form of the mass conservation

$$
\left.\frac{\partial \rho}{\partial t}\right|_{\chi}+\frac{\partial \rho}{\partial x_{j}} c_{j}=-\rho \frac{\partial v_{j}}{x_{j}}
$$

Using the same rule, the ALE form of the momentum and energy conservations can also be obtained, as written in Equations (3-15b) and (3-15c), respectively.

$$
\begin{aligned}
& \rho\left(\left.\frac{\partial v_{i}}{\partial t}\right|_{\chi}+\frac{\partial v_{i}}{\partial x_{j}} c_{j}\right)=\frac{\partial \sigma_{j i}}{\partial x_{j}}+\rho b_{i} \\
& \rho\left(\left.\frac{\partial w^{\text {int }}}{\partial t}\right|_{\chi}+\frac{\partial w^{\text {int }}}{\partial x_{j}} c_{j}\right)=D_{j i} \sigma_{j i}-\frac{\partial q_{i}}{\partial x_{i}}+\rho s
\end{aligned}
$$

\subsubsection{Constitutive Equation}

The material behavior during FSW is modeled as hypoelastic-plastic. In this model, the additive decomposition of the rate of deformation into elastic and plastic parts is assumed:

$$
D_{i j}=D_{i j}^{e}+D_{i j}^{p}
$$


where

$$
D_{i j}=\frac{1}{2}\left(\frac{\partial v_{i}}{\partial x_{j}}+\frac{\partial v_{j}}{\partial x_{i}}\right)
$$

The constitutive equation is commonly expressed in terms of the objective rate of the Cauchy stress and the total rate of deformation

$$
\sigma_{i j}^{\nabla}=C_{i j k l}^{\sigma} D_{k l}
$$

where $\sigma_{i j}^{\nabla}$ is the objective rate of the Cauchy stress; $C_{i j k l}^{\sigma}$ is the elastoplastic tangent modulus

One popular objective rate is the Jaumann rate

$$
\sigma_{i j}^{\nabla J}=\frac{d \sigma_{i j}}{d t}-W_{i k} \sigma_{k j}-\sigma_{i k} W_{k j}^{T}
$$

where

$$
W_{i j}=\frac{1}{2}\left(\frac{\partial v_{i}}{\partial x_{j}}-\frac{\partial v_{j}}{\partial x_{i}}\right)
$$

Substituting Equation (3-19) into Equation (3-18) and rearranging obtains

$$
\frac{d \sigma_{i j}}{d t}=\underbrace{C_{i j k l}^{\sigma J} D_{k l}}_{\text {material }}+\underbrace{W_{i k} \sigma_{k j}+\sigma_{i k} W_{k j}^{T}}_{\text {rotation }}
$$

In the above, the material derivative of the Cauchy stress consists of two parts: the rate of change due to material response which is reflected in the objective rate, and the change of stress due to rotation, which corresponds to the last two terms.

To get the ALE form of the constitutive equation, applying Equation (3-13) to Equation (3-21) becomes

$$
\left.\frac{\partial \sigma_{i j}}{\partial t}\right|_{\chi}+\frac{\partial \sigma_{i j}}{\partial x_{j}} c_{j}=C_{i j k l}^{\sigma J} D_{k l}+W_{i k} \sigma_{k j}+\sigma_{i k} W_{k j}
$$


It is now that all the equations have been prepared to solve the problem of FSW by the use of ALE formulations. However, if the mechanical effects are uncoupled from thermal effect, the mass equation (3-15a), and the momentum equation (3-15b) can be solved independently from the energy equation (3-15c). The mass and momentum equations are solved with the constitutive equation (3-22) as well as the following boundary and initial conditions to find $\rho(\chi, t), \mathbf{u}(\chi, t), \hat{\mathbf{u}}(\chi, t), \sigma(\chi, t)$.

Mass $\left.\quad \frac{\partial \rho}{\partial t}\right|_{\chi}+\frac{\partial \rho}{\partial x_{j}} c_{j}=-\rho \frac{\partial v_{j}}{x_{j}}$

Momentum $\rho\left(\left.\frac{\partial v_{i}}{\partial t}\right|_{\chi}+\frac{\partial v_{i}}{\partial x_{j}} c_{j}\right)=\frac{\partial \sigma_{j i}}{\partial x_{j}}+\rho b_{i}$

Constitutive $\left.\frac{\partial \sigma_{i j}}{\partial t}\right|_{\chi}+\frac{\partial \sigma_{i j}}{\partial x_{j}} c_{j}=C_{i j k l}^{\sigma J} D_{k l}+W_{i k} \sigma_{k j}+\sigma_{i k} W_{k j}$

The boundary conditions are:

$$
n_{j}(\chi, t) \sigma_{j i}(\chi, t)=\bar{t}_{i}(\chi, t) \text { on } \Gamma_{t_{i}} \quad v_{i}(\chi, t)=\bar{v}_{i}(\chi, t) \text { on } \Gamma_{v_{i}}
$$

where $\bar{t}_{i}$ is the boundary tractions; $\bar{v}_{i}$ is the boundary velocities; $\Gamma_{t_{i}}, \Gamma_{v_{i}}$ is the traction and velocity boundary of the spatial domain, respectively.

The initial conditions are:

$$
\sigma(\mathbf{X}, 0)=\sigma_{0}(\mathbf{X}) \quad \mathbf{v}(\mathbf{X}, 0)=\mathbf{v}_{\mathbf{0}}(\mathbf{X})
$$

After obtaining the ALE form of the PDEs of the problem its weak form is derived in section 3.3.1. Then all dependent variables in the weak form are approximated as functions of the element coordinates in section 3.3.2 and the relevant finite element matrix equations are derived in section 3.3.3. 


\subsection{Finite Element Method}

\subsubsection{Weak Forms}

After defining the PDEs of mass, momentum and constitutive in ALE formulations, their weak forms need to be developed. The weak form is obtained by integrating the product of a test function with the equation over the current spatial domain.

\section{Weak Form of the Mass Equation}

The weak form of the mass equation is obtained by multiplying Equation (3-15a) with a test function $\delta \rho$ and integrating over the current spatial domain $\Omega$.

$$
\left.\int_{\Omega} \delta \rho \frac{\partial \rho}{\partial t}\right|_{\chi} d \Omega+\int_{\Omega} \delta \rho \frac{\partial \rho}{\partial x_{j}} c_{j} d \Omega+\int_{\Omega} \delta \rho \rho \frac{\partial v_{j}}{x_{j}} d \Omega=0
$$

\section{Weak Form of the Momentum Equation}

The weak form of the momentum equation is obtained by multiplying Equation (3-15b) with a test function $\delta v_{i}$

$$
\left.\int_{\Omega} \delta v_{i} \rho \frac{\partial v_{i}}{\partial t}\right|_{\chi} d \Omega+\int_{\Omega} \delta v_{i} \rho \frac{\partial v_{i}}{\partial x_{j}} c_{j} d \Omega=\int_{\Omega} \delta v_{i} \frac{\partial \sigma_{j i}}{\partial x_{j}} d \Omega+\int_{\Omega} \delta v_{i} \rho b_{i} d \Omega
$$

Integrating the first term in the right hand $\int_{\Omega} \delta v_{i} \frac{\partial \sigma_{j i}}{\partial x_{j}} d \Omega$ by parts yields the weak form of the momentum equation 


$$
\begin{aligned}
& \left.\int_{\Omega} \delta v_{i} \rho \frac{\partial v_{i}}{\partial t}\right|_{\chi} d \Omega+\int_{\Omega} \delta v_{i} \rho \frac{\partial v_{i}}{\partial x_{j}} c_{j} d \Omega \\
& =-\int_{\Omega} \frac{\partial\left(\delta v_{i}\right)}{\partial x_{j}} \sigma_{i j} d \Omega+\int_{\Omega} \delta v_{i} \rho b_{i} d \Omega+\int_{\Gamma} \delta v_{i} \bar{t}_{i} d \Gamma
\end{aligned}
$$

\section{Weak Form of the Constitutive Equation}

As for the mass and momentum equations, the weak form of the constitutive equation is obtained by multiplying Equation (3-22) by a test function $\delta \sigma_{i j}$

$$
\begin{aligned}
& \left.\int_{\Omega} \delta \sigma_{i j} \frac{\partial \sigma_{i j}}{\partial t}\right|_{\chi} d \Omega+\int_{\Omega} \delta \sigma_{i j} \frac{\partial \sigma_{i j}}{\partial x_{j}} c_{j} d \Omega \\
& =\int_{\Omega} \delta \sigma_{i j} C_{i j k l}^{\sigma J} D_{k l} d \Omega+\int_{\Omega} \delta \sigma_{i j}\left(W_{i k} \sigma_{k j}+\sigma_{i k} W_{k j}\right) d \Omega
\end{aligned}
$$

\subsubsection{Finite Element Approximations}

The velocity, density and stress in the weak form of the mass, momentum and constitutive equations will be approximated as functions of the element coordinates.

\section{Approximation of Velocity}

The reference (i.e. ALE) domain is subdivided into elements and for element $e$ the ALE coordinates are given by

$$
\chi\left(\xi^{e}\right)=\chi_{I} N_{I}\left(\xi^{e}\right)
$$

where $\xi^{e}$ are the element coordinates of element $e, I$ stands for the node I, $\chi_{I}$ is the ALE coordinate of node $I, N_{I}\left(\xi^{e}\right)$ are the shape functions for the velocity. 
Based on Equation (3-29), the spatial position of point $\chi$ given by Equation (3-2) is approximately computed by

$$
\mathbf{x}=\boldsymbol{\Phi}(\chi, t)=\mathbf{\Phi}\left(\chi\left(\xi^{e}\right), t\right)=\mathbf{x}_{I}(t) N_{I}\left(\xi^{e}\right)
$$

where $\mathbf{x}_{I}(t)$, are the motions of the nodes.

So the mesh velocity given by Equation (3-5b) is approximately by

$$
\hat{\mathbf{v}}=\frac{\partial \Phi(\chi, t)}{\partial t}=\frac{\partial \mathbf{x}_{I}(t)}{\partial t} N_{I}\left(\xi^{e}\right)=\hat{\mathbf{v}}_{I}(t) N_{I}\left(\xi^{e}\right)
$$

where $\hat{\mathbf{v}}_{I}(t)$ is the mesh velocity of node $I$.

The material velocity in Equation (3-4b) is interpolated as

$$
\mathbf{v}=\mathbf{v}_{I}(t) N_{I}\left(\xi^{e}\right)
$$

In similar way, the convective velocity $\mathbf{c}$ is interpolated as

$$
\mathbf{c}=\mathbf{c}_{I}(t) N_{I}\left(\xi^{e}\right)
$$

where $\mathbf{c}_{I}(t)$ is the convective velocity of node $I$

So, substituting Equations (3-31), (3-32) and (3-33) into Equation (3-9) yields

$$
\mathbf{c}=\mathbf{c}_{I}(t) N_{I}\left(\xi^{e}\right)=\left(\mathbf{v}_{I}(t)-\hat{\mathbf{v}}_{I}(t)\right) N_{I}\left(\xi^{e}\right)
$$

\section{Approximation of Density and Stress}

The density and stress are approximated by

$$
\begin{aligned}
& \rho\left(\xi^{e}, t\right)=\rho_{I}(t) N_{I}^{\rho}\left(\xi^{e}\right) \\
& \sigma\left(\xi^{e}, t\right)=\sigma_{I}(t) N_{I}^{\sigma}\left(\xi^{e}\right)
\end{aligned}
$$

where $N_{I}^{\rho}\left(\xi^{e}\right), N_{I}^{\sigma}\left(\xi^{e}\right)$ are sets of the shape functions for the density and stress, and $\rho_{I}(t), \sigma_{I}(t)$ are the mass density and the Cauchy stress of node $I$, respectively. 


\subsubsection{Finite Element Matrix Equations}

With all the equations obtained from the previous section, the material time derivative of velocity $\frac{d \mathbf{v}}{d t}$, density $\frac{d \rho}{d t}$ and stress $\frac{d \sigma}{d t}$, can be approximately calculated. Then they are substituted into the weak form equations to derive the finite element matrix equations for mass, momentum and stress as follows.

$$
\underline{\text { Mass }} \quad \mathbf{M}^{\rho} \frac{d \rho}{d t}+\mathbf{L}^{\rho} \boldsymbol{\rho}+\mathbf{K}^{\rho} \rho=0
$$

Where $\mathbf{M}^{\rho}, \mathbf{L}^{\rho}, \mathbf{K}^{\rho}$ are capacitance, transport and divergence matrices for mass density, respectively.

$$
\begin{aligned}
& \mathbf{M}^{\rho}=\left[M_{I J}^{\rho}\right]=\int_{\Omega} \bar{N}_{I}^{\rho} N_{J}^{\rho} d \Omega \quad \quad \mathbf{L}^{\rho}=\left[L_{I J}^{\rho}\right]=\int_{\Omega} \bar{N}_{I}^{\rho} c_{i} \frac{d N_{J}^{\rho}}{d x_{i}} d \Omega \\
& \mathbf{K}^{\rho}=\left[K_{I J}^{\rho}\right]=\int_{\Omega} \bar{N}_{I}^{\rho} \frac{\partial v_{i}}{\partial x_{i}} N_{J}^{\rho} d \Omega \quad \rho=\left[\rho_{J}\right] \quad \frac{d \rho}{d t}=\left[\frac{d \rho_{J}}{d t}\right]
\end{aligned}
$$

$\underline{\text { Momentum }} \quad \mathbf{M} \frac{d \mathbf{v}}{d t}+\mathbf{L v}+\mathbf{f}^{\text {int }}=\mathbf{f}^{\text {ext }}$

Where $\mathbf{M}$ and $\mathbf{L}$ are generalized mass and convective matrices, respectively, for velocity under a reference description; while $\mathbf{f}^{\text {int }}$ and $\mathbf{f}^{\text {ext }}$ are the internal and external force vectors respectively. It is noted that the mass matrix is not constant in time since the density and domain vary with time.

$$
\begin{aligned}
& \mathbf{M}=\mathbf{I}\left[M_{I J}\right]=\mathbf{I}\left(\int_{\Omega} \rho \bar{N}_{I} N_{J} d \Omega\right) \quad \mathbf{L}=\mathbf{I}\left[L_{I J}\right]=\mathbf{I}\left(\int_{\Omega} \rho \bar{N}_{I} c_{i} \frac{d N_{J}}{d x_{i}} d \Omega\right) \\
& \mathbf{f}^{\text {int }}=\left[f_{i I}^{\text {int }}\right]=\int_{\Omega} \frac{d \bar{N}_{I}}{d x_{j}} \sigma_{i j} d \Omega \quad \mathbf{f}^{\text {ext }}=\left[f_{i I}^{\text {ext }}\right]=\int_{\Omega} \rho \bar{N}_{I} b_{i} d \Omega+\int_{\Gamma_{t_{i}}} \bar{N}_{I} \bar{t}_{i} d \Gamma
\end{aligned}
$$




$$
\mathbf{v}=\left[v_{J}\right] \quad \frac{d \mathbf{v}}{d t}=\left[\frac{d v_{J}}{d t}\right]
$$

$\underline{\text { Constitutive }} \quad \mathbf{M}^{\sigma} \frac{d \sigma}{d t}+\mathbf{L}^{\sigma} \sigma=\mathbf{z}$

Where $\mathbf{M}^{\sigma}$ and $\mathbf{L}^{\sigma}$ are the generalized mass and convective matrices for stress, respectively. $\mathbf{z}$ is the generalized stress vector

$$
\begin{gathered}
\mathbf{M}^{\sigma}=\mathbf{I}\left[M_{I J}^{\sigma}\right]=\mathbf{I}\left(\int_{\Omega} \bar{N}_{I}^{\sigma} N_{J}^{\sigma} d \mathbf{\Omega}\right) \quad \mathbf{L}^{\sigma}=\mathbf{I}\left[L_{I J}^{\sigma}\right]=\mathbf{I}\left(\int_{\Omega} \bar{N}_{I}^{\sigma} c_{i} \frac{d N_{J}^{\sigma}}{d x_{i}} d \Omega\right) \\
\mathbf{z}=\left[z_{I}\right]=\int_{\Omega} \bar{N}_{I}^{\sigma} C_{i j k l}^{\sigma J} D_{k l} d \Omega+\int_{\Omega} \bar{N}_{I}^{\sigma}\left(W_{i k} \sigma_{k j}+\sigma_{i k} W_{k j}\right) d \Omega \quad \sigma=\left[\sigma_{J}\right]=\left\lfloor\sigma_{i j J}\right\rfloor
\end{gathered}
$$

Equations (3-37), (3-38) and (3-39) are the ordinary differential equations in time. They are called semi-discrete since they have been discretized in space but not time. 


\subsection{Solution Procedure}

Equations (3-15a), (3-15b) and (3-22) contain convective terms that account for the relative motion between the mesh and material, causing numerical solutions to these equations are much more difficult than Lagrangian equations (where the relative velocity is zero). Generally, there are two ways to implement ALE equations, namely full coupled and operator split as reviewed in the chapter 2. In this research, the operator split approach is applied to solving those equations in two steps: a Lagrangian step and an advection step. In the Lagrangian step, the mesh moves with the material and the mesh velocity is equal to the material velocity, so a standard Lagrangian problem is solved. Sequentially, an advection step is performed and takes into consideration the difference between the material and mesh velocities. In this step, first the distorted mesh is smoothed, and then the solution is mapped from the distorted mesh to the smoothed mesh.

\subsubsection{Operator Split}

To show the operator split approach how to work in ALE formulations, Equations (3-15a), (3-15b) and (3-22) are written as a general form (3-40) since they have an identical structure.

$$
\left.\frac{\partial \phi}{\partial t}\right|_{\chi}+\frac{\partial \phi}{\partial \mathbf{x}} \cdot \mathbf{c}=f
$$

where $\phi$ is a field variable which represents density, velocity and stress; c is the convective velocity; $f$ is the source term. 
Based on operator split, Equation (3-40) is broken into two equations (3-41) and $(3-42)$

$$
\begin{aligned}
& \left.\frac{\partial \phi}{\partial t}\right|_{\chi}=f, \quad \mathbf{c}=0 \\
& \left.\frac{\partial \phi}{\partial t}\right|_{\chi}+\frac{\partial \phi}{\partial \mathbf{x}} \cdot \mathbf{c}=0, \quad f=0
\end{aligned}
$$

\subsubsection{Lagrangian step}

The solution to Equation (3-41) is obtained by the use of explicit time integration scheme. The explicit time integration scheme will be discussed in Chapter 4.

$$
\phi_{n+1}^{L}=\phi_{n}+\left.\frac{\partial \phi_{n}}{\partial t}\right|_{\mathbf{x}} \Delta t \text { with }\left.\quad \frac{\partial \phi_{n}}{\partial t}\right|_{\mathbf{x}}=f
$$

By the use of Equation (3-43) and the finite element matrix equations (3-37), (338) and (3-39), the Lagrangian solutions of density, velocity and stress are obtained as

$$
\begin{array}{ll}
\rho_{n+1}^{L}=\rho_{n}+\left.\frac{\partial \rho_{n}}{\partial t}\right|_{\mathbf{x}} \Delta t \text { with } & \left.\frac{\partial \rho_{n}}{\partial t}\right|_{\mathbf{x}}=-\left(\mathbf{M}_{n}^{\rho}\right)^{-1} \mathbf{K}_{n}^{\rho} \boldsymbol{\rho}_{n}^{L} \\
\mathbf{v}_{n+1}^{L}=\mathbf{v}_{n}+\left.\frac{\partial \mathbf{v}_{n}}{\partial t}\right|_{\mathbf{x}} \Delta t \text { with } & \left.\frac{\partial \mathbf{v}_{n}}{\partial t}\right|_{\mathbf{x}}=\mathbf{M}_{n}^{-1}\left(\mathbf{f}_{n}^{\text {ext }}-\mathbf{f}_{n}^{\text {int }}\right) \\
\sigma_{n+1}^{L}=\sigma_{n}+\left.\frac{\partial \sigma_{n}}{\partial t}\right|_{\mathbf{x}} \Delta t \text { with } & \left.\frac{\partial \sigma_{n}}{\partial t}\right|_{\mathbf{x}}=-\left(\mathbf{M}_{n}^{\sigma}\right)^{-\mathbf{1}} \mathbf{z}_{n}
\end{array}
$$




\subsubsection{Advection step}

In this step, the distorted Lagrangian mesh is smoothed first. As a result, the mesh velocity is not equal to the material velocity and a convective velocity $\mathbf{c}$ is produced. Then the Lagrangian solution is mapped onto the new mesh. The final solution is given by

$$
\phi_{n+1}=\phi_{n+1}^{L}+\left.\frac{\partial \phi_{n+1}^{L}}{\partial t}\right|_{\chi} \Delta t
$$

where $\left.\frac{\partial \phi_{n+1}^{L}}{\partial t}\right|_{\chi}$ is determined by Equation (3-42).

\subsubsection{Mesh Smoothing}

After the Lagrangian step, the mesh may be highly distorted and need to be adjusted. Two steps are taken for smoothing such mesh: 1) deciding which nodes to move, 2) where to move the nodes. It is noted that the connectivity of elements is not changed in an ALE calculation. That means the boundary nodes should remain on the boundary while the interior nodes are moved in order to reduce element deformations.

\section{Deciding Which Nodes to Move}

Before the mesh is smoothed, a decision has to be made on which nodes to move first. Two criteria are applied to selecting which nodes to move, namely shear and volumetric element distortion. Shear distortion is quantified by calculating the angle $(\theta)$ formed by the element sides surrounding a node (see Fig.3-3). Then, the calculated angle is compared to the user specified minimum angle. When the angle is below the specified 
one, the node is flagged for being moved. The other approach, volumetric distortion is detected by calculating the volume $(V)$ of the elements connected to a node (see Fig.3-3) and taking the ratio of the smallest element volume to the largest element volume. The ratio falls below the user specified level, the node is flagged for being moved.

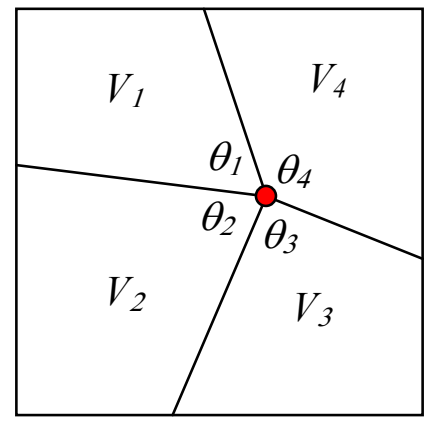

Figure 3-3 Evaluating the Angle ( $\theta)$ and Volume (V) around a Node (Red Point)

\section{Moving Nodes}

The widely used method for moving the nodes is called equipotential relaxation stencil. This method tends to equalize element volumes as well as the angles formed by element sides at each node.

This stencil is derived from a finite difference representation of the Laplace equation $\nabla^{2} f=0$. In three dimensions $\mathrm{x}, \mathrm{y}, \mathrm{z}$, we define curvilinear coordinates $\xi, \eta, \zeta$ which satisfy Laplace equation

$$
\begin{aligned}
& \nabla^{2} \xi=0 \\
& \nabla^{2} \eta=0 \\
& \nabla^{2} \zeta=0
\end{aligned}
$$

The inverted form of Equations (3-48) is given by

$$
\alpha_{1} \vec{r}_{\xi \xi}+\alpha_{2} \vec{r}_{\eta \eta}+\alpha_{3} \vec{r}_{\zeta \zeta}+2 \beta_{1} \vec{r}_{\xi \eta}+2 \beta_{2} \vec{r}_{\eta \zeta}+2 \beta_{3} \vec{r}_{\zeta \xi}=0
$$


where $\vec{r}=x \hat{i}+y \hat{j}+z \hat{k}$, and coefficients $\alpha_{1}, \alpha_{2}, \alpha_{3}, \beta_{1}, \beta_{2}, \beta_{3}$ are dependent on $\vec{r}_{\xi}, \vec{r}_{\eta}, \vec{r}_{\zeta}$, A variable subscript indicates differentiation with respect to that variable.

The difference of Equation (3-49) is taken in a cube in the rectangular $\xi, \eta, \zeta$ space with unit spacing between the coordinate surfaces, using subscript $i$ to represent the $\xi$ direction, $\mathrm{j}$ the $\eta$ direction, and $\mathrm{k}$ the $\zeta$ direction, as shown in Fig.3-4.

Using central differencing, the finite difference approximations are obtained for the coordinate derivatives $\vec{r}_{\xi}, \vec{r}_{\eta}, \vec{r}_{\zeta}, \vec{r}_{\xi \xi}, \vec{r}_{\eta \eta}, \vec{r}_{\zeta \zeta}, \vec{r}_{\xi \eta}, \vec{r}_{\eta \zeta}, \vec{r}_{\zeta \xi}$ and then they are substituted into Equation (3-49) to solve the coordinates of the central point as a weighted mean of its 18 nearest neighbors. The weight coefficients are given in Table 3-1.

$$
x=\frac{\sum_{m} \omega_{m} x_{m}}{\sum_{m} \omega_{m}}, \quad y=\frac{\sum_{m} \omega_{m} y_{m}}{\sum_{m} \omega_{m}}, \quad z=\frac{\sum_{m} \omega_{m} z_{m}}{\sum_{m} \omega_{m}}
$$

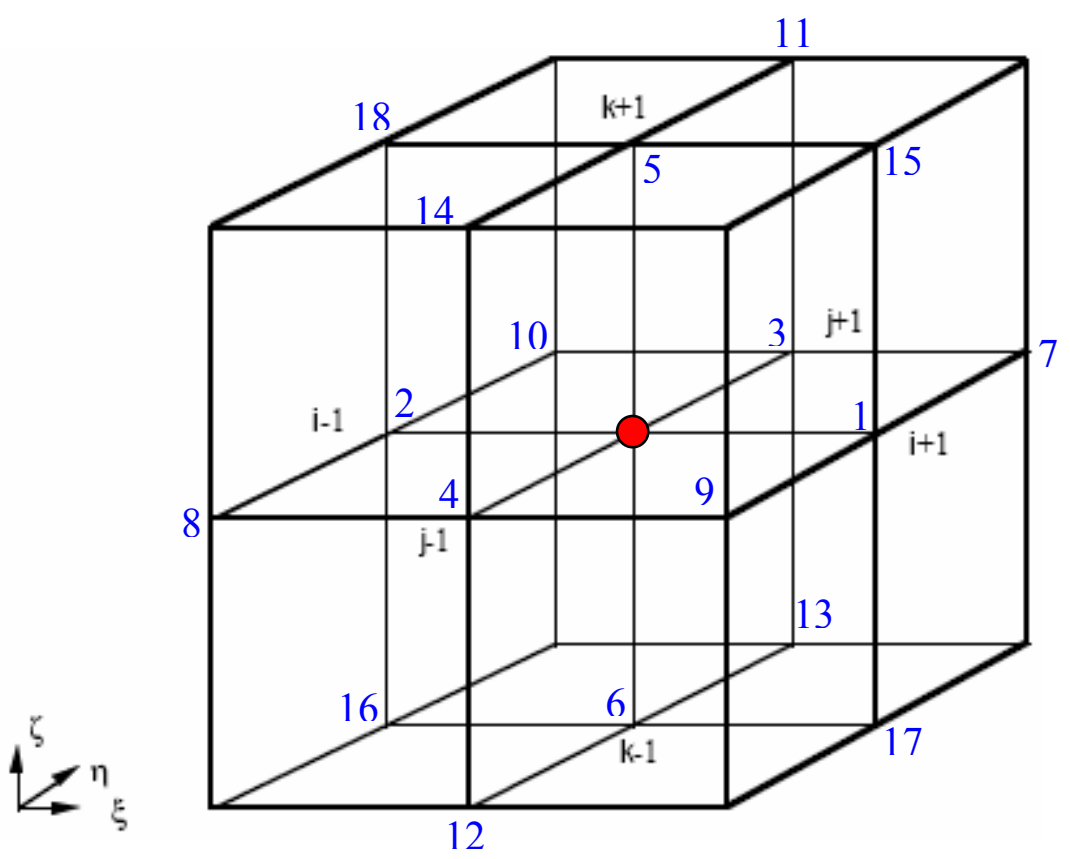

Figure 3-4 The Numbering of the Points around the Central Point (Red) for the Equipotential Relaxation Stencil (Hallquist [26]) 
Table 3-1 3D Rezoning Weight Coefficients (Hallquist [26])

\begin{tabular}{c|ccccccccc}
\hline $\mathrm{m}$ & 1 & 2 & 3 & 4 & 5 & 6 & 7 & 8 & 9 \\
$\omega_{\mathrm{m}}$ & $\alpha_{1}$ & $\alpha_{1}$ & $\alpha_{2}$ & $\alpha_{2}$ & $\alpha_{3}$ & $\alpha_{3}$ & $\frac{\beta_{1}}{2}$ & $\frac{\beta_{1}}{2}$ & $-\frac{\beta_{1}}{2}$ \\
\hline \hline $\mathrm{m}$ & 10 & 11 & 12 & 13 & 14 & 15 & 16 & 17 & 18 \\
$\omega_{\mathrm{m}}$ & $-\frac{\beta_{1}}{2}$ & $\frac{\beta_{2}}{2}$ & $\frac{\beta_{2}}{2}$ & $-\frac{\beta_{2}}{2}$ & $-\frac{\beta_{2}}{2}$ & $\frac{\beta_{3}}{2}$ & $\frac{\beta_{3}}{2}$ & $-\frac{\beta_{3}}{2}$ & $-\frac{\beta_{3}}{2}$ \\
\hline
\end{tabular}

\section{Application to the ALE Formulation}

As a result of the mesh smoothing, the mesh nodes have the new spatial position $\mathbf{x}_{n+1}$. The new mesh velocity is then obtained by $\hat{\mathbf{v}}_{n+1}=\left(\mathbf{x}_{n+1}-\mathbf{x}_{n}\right) / \Delta t$. The mesh and material velocities are not equal any more. Thus the convective velocity $\mathbf{c}_{n+1}$ is produced as given in Equation (3-9). This convective velocity will be used in the next step.

$$
\mathbf{c}_{n+1}=\mathbf{v}_{n+1}^{L}-\hat{\mathbf{v}}_{n+1}
$$

where $\mathbf{v}_{n+1}^{L}$ is the material velocity from the Lagrangian step; $\hat{\mathbf{v}}_{n+1}$ is the mesh velocity from the mesh smoothing. 


\subsubsection{Remap of Solution Variables}

After the distorted mesh is smoothed, remap step is taken in which the solution variables calculated during the Lagrangian step must be remapped from the distorted mesh to the new mesh which is generated by the mesh smoothing. The convective effect is considered in this step. Based on Equations (3-42) and (3-47), the final solutions of density $\rho_{n+1}$, velocity $\mathbf{v}_{\mathbf{n}+\mathbf{1}}$ and stress $\sigma_{n+1}$ is calculated from the solutions of the Lagrangian step $\rho_{n+1}^{L}, \mathbf{v}_{n+1}^{L}$ and $\sigma_{n+1}^{L}$ given in Equations (3-44), (3-45) and (3-46), respectively.

The algorithms for integrating Equation (3-42) are called advection algorithms. These algorithms are required to be a spatially almost second-order accurate, stable, conservative and monotonic. Conservativity requires the integral of all the solution variables over the material domain should remain unchanged by the remap. And monotonicity requires that range of the solution variables does not increase during the remap step. Two such algorithms namely Donor Cell and Van Leer MUSCL are implemented to integrate Equation (3-42). Both algorithms are based on Godunov method and assume that the advected solution variables are centered within the element. But the Donor Cell algorithm is a first-order accurate while the Van Leer MUSCL algorithm is a two-order accurate. It should be noted these two algorithms are used to advect the density and stress which are centered in the elements while they must be modified to advect the node-centered velocity. Next, the Donor cell and Van Leer MUSCL algorithms are discussed. 


\section{The Donor Cell Algorithm}

The donor cell algorithm is a first order Godunov method and assumes that the distribution of $\phi_{n+1}^{L}$ is piecewise constant over an element after the Lagrangian step.

First, Equation (3-42) is transformed to the following equation using the definition of $\mathbf{Y}=\phi_{n+1}^{L} \mathbf{c}_{n+1}$

$$
\left.\frac{\partial \phi_{n+1}^{L}}{\partial t}\right|_{\chi}+\frac{\partial \mathbf{Y}}{\partial \mathbf{x}}-\frac{\partial \mathbf{c}_{n+1}}{\partial \mathbf{x}} \phi_{n+1}^{L}=0
$$

since $\frac{\partial \mathbf{Y}}{\partial \mathbf{x}}=\frac{\partial\left(\phi_{n+1}^{L} \mathbf{c}_{n+1}\right)}{\partial \mathbf{x}}=\frac{\partial \phi_{n+1}^{L}}{\partial \mathbf{x}} \cdot \mathbf{c}_{n+1}+\frac{\partial \mathbf{c}_{n+1}}{\partial \mathbf{x}} \phi_{n+1}^{L}$

The weak form of Equation (3-52) is obtained by multiplying Equation (3-52) by a test function $\delta \phi_{n+1}^{L}$. Here, the equation to the spatial domain $\Omega^{e}$ of one element $e$ is applied

$$
\left.\int_{\Omega^{e}} \delta \phi_{n+1}^{L} \frac{\partial \phi_{n+1}^{L}}{\partial t}\right|_{\chi} d \Omega+\int_{\Omega^{e}} \delta \phi_{n+1}^{L} \frac{\partial \mathbf{Y}}{\partial \mathbf{x}} d \Omega-\int_{\Omega^{e}} \delta \phi_{n+1}^{L} \frac{\partial \mathbf{c}_{n+1}}{\partial \mathbf{x}} \phi_{n+1}^{L} d \Omega=0
$$

Since $\delta \phi_{n+1}^{L}$ is arbitrary and $\phi_{n+1}^{L}$ is assumed to be constant over an element which implies also $\frac{\partial \phi_{n+1}^{L}}{\partial t}$ is constant, thus

$$
\frac{\partial \phi_{n+1}^{L}}{\partial t} \int_{\Omega^{e}} d \boldsymbol{\Omega}+\int_{\Omega^{e}} \frac{\partial \mathbf{Y}}{\partial \mathbf{x}} d \boldsymbol{\Omega}-\phi_{n+1}^{L} \int_{\Omega^{e}} \frac{\partial \mathbf{c}_{n+1}}{\partial \mathbf{x}} d \boldsymbol{\Omega}=0
$$

Applying Gauss's theorem to the second and third term yields

$$
\begin{aligned}
& \int_{\Omega^{e}} \frac{\partial \mathbf{Y}}{\partial \mathbf{x}} d \boldsymbol{\Omega}=\int_{\Gamma^{e}}\left(\mathbf{n}^{e} \cdot \mathbf{Y}\right) d \Gamma \\
& \int_{\Omega^{e}} \frac{\partial \mathbf{c}_{n+1}}{\partial \mathbf{x}} d \boldsymbol{\Omega}=\int_{\Gamma^{e}}\left(\mathbf{n}^{e} \cdot \mathbf{c}_{n+1}\right) d \Gamma
\end{aligned}
$$


where $\Gamma^{e}$ is the boundary of $\Omega^{e}$ and $\mathbf{n}^{e}$ is the outward normal to the boundary of element $e$

Substituting Equation (3-55) into (3-54) and with $\mathbf{Y}=\phi_{n+1}^{L} \mathbf{c}_{n+1}$ and then rearranging produces the following equation

$$
\frac{\partial \phi_{n+1}^{L}}{\partial t} \int_{\Omega^{e}} d \Omega=-\int_{\Gamma^{e}}\left(\mathbf{n}^{e} \cdot\left(\phi_{n+1}^{L} \cdot \mathbf{c}_{n+1}\right)\right) d \Gamma+\phi_{n+1}^{L} \int_{\Gamma^{e}}\left(\mathbf{n}^{e} \cdot \mathbf{c}_{n+1}\right) d \Gamma
$$

By splitting the boundary integrals in Equation (3-56) into its element sides and by the use of the obvious result $\int_{\Omega^{e}} d \Omega=V$ the following results are obtained

$$
\frac{\partial \phi_{n+1}^{L}}{\partial t}=-\frac{1}{V} \sum_{s=1}^{N_{s}}\left[\left(\int_{\Gamma^{e}} \mathbf{n}^{e} \cdot \mathbf{c}_{n+1} d \Gamma\right)\left(\phi_{s, n+1}^{L}-\phi_{n+1}^{L}\right)\right]
$$

where $\phi_{s, n+1}^{L}$ is $\phi_{n+1}^{L}$ along the side $s$ of the element under consideration, $N_{s}$ is the total number of sides of the element and $V$ is the volume of the element.

Now we introduce the flux of convective velocity

$$
f_{s}=\int_{\Gamma^{e}} \mathbf{n}^{e} \cdot \mathbf{c}_{n+1} d \Gamma
$$

Since $\phi_{n+1}^{L}$ is constant inside the element and $\phi_{s, n+1}^{L}$ is constant along each side $s$ of the element. Now the total flux along one side can be introduced as

$$
F_{s}=f_{s} \phi_{s, n+1}^{L}
$$

To calculate $\phi_{s, n+1}^{L}$, both $\phi_{n+1}^{L}$ in the element and $\phi_{n+1}^{C L}$ in the element contiguous to the present one across the side $s$ are needed. $\phi_{n+1}^{C L}$ is illustrated in Fig.3-5. Then $\phi_{s, n+1}^{L}$ can be calculated as

$$
\phi_{s, n+1}^{L}=\frac{1}{2}\left[\left(1+\operatorname{sign}\left(f_{s}\right)\right) \cdot \phi_{n+1}^{L}+\left(1-\operatorname{sign}\left(f_{s}\right)\right) \cdot \phi_{n+1}^{C L}\right]
$$


Substituting Equations (3-60) and (3-58) into Equation (3-57) yields the generalized time integration equation for each element

$$
\frac{\partial \phi_{n+1}^{L}}{\partial t}=-\frac{1}{2 V} \sum_{s=1}^{N_{s}}\left[f_{s}\left(1-\operatorname{sign}\left(f_{s}\right)\right)\left(\phi_{n+1}^{C L}-\phi_{n+1}^{L}\right)\right]
$$

The above equation then can be used to calculate the final solution to Equation (3-40) for the update of function $\phi$.

By the use of Equations (3-43) and (3-61), the final solution to Equation (3-40) based on the first order Godunov method is

$$
\phi_{n+1}=\phi_{n+1}^{L}-\frac{1}{2 V} \sum_{s=1}^{N_{s}}\left[f_{s}\left(1+\operatorname{sign}\left(f_{s}\right)\right)\left(\phi_{n+1}^{C L}-\phi_{n+1}^{L}\right)\right]
$$

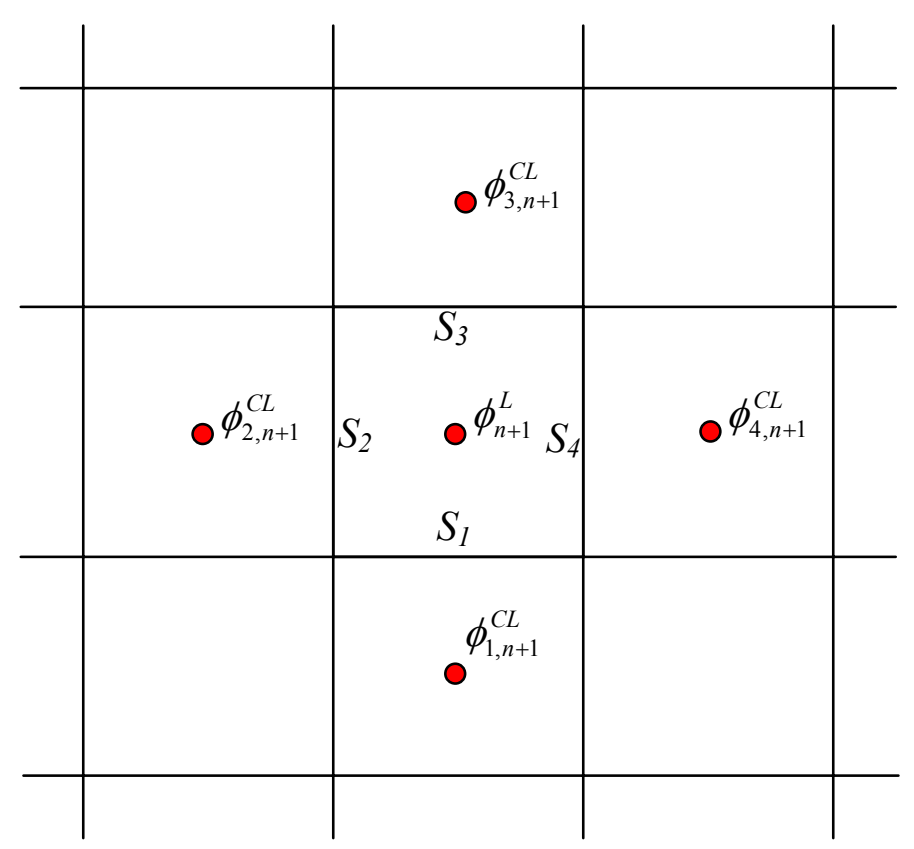

Figure 3-5 Illustration of the Different $\phi_{n+1}^{L}$ 's (Linder [37]) 


\section{The Van Leer MUSCL Algorithm}

The Van Leer MUSCL (Monotone Upwind Schemes for Conservation Laws) algorithm is a higher order Godunov method. It was developed to obtain a second order accurate result. The algorithm replaces the piecewise constant distribution over the element with an assumed linear distribution, $\phi_{n+1}^{L}(\mathbf{x})$ that is subject to an element level conservation constraint. The value of $\phi_{n+1}^{L}$ at the element centroid is regarded in this context as the average value of $\phi_{n+1}^{L}(\mathbf{x})$ over the element

$$
\phi_{n+1}^{L}=\frac{1}{\Omega^{e}} \int_{\Omega^{e}} \phi_{n+1}^{L}(\mathbf{x}) d \Omega
$$

Due to the assumed linear distribution $\phi_{n+1}^{L}(\mathbf{x})$, some parts of the solution might exceed the extreme values at the adjacent elements. Monotonicity constraint is imposed on $\phi_{n+1}^{L}(\mathbf{x})$ to determine the range $\left[\phi_{n+1}^{L}{ }^{\min }, \phi_{n+1}^{L}{ }^{\max }\right]$.

\section{Advection of Node-centered Variables}

The velocity is stored at the nodes, as opposed to element-centered variables. Momentum is advected instead of the velocity to ensure that momentum is conserved. The element-centered advection algorithm must be modified to advect the node-centered momentum. For advection momentum, a new mesh that is staggered with respect to original mesh is defined so that the original nodes become the centroids of the new elements. Then the advection algorithms discussed above are applied to this new mesh. Another approach is to construct an auxiliary set of element-centered variables from the momentum, advect them, and then reconstruct the new velocities from the auxiliary variables. 


\section{CHAPTER 4 FSW SIMULATION}

\subsection{Introduction}

In this chapter, the material flow during FSW is simulated using ALE formulations. For the purpose of comparison, the process is also simulated with Lagrangian formulations. It will be shown that the problem of FSW is difficult to handle with Lagrangian formulations. However it does not present any difficulty in ALE formulations.

The general-purpose finite element code LS-DYNA is used to carry out the simulations. LS-DYNA was developed by Lawrence Livermore Software Technology Corporation. It is applied to analyzing the large deformation dynamic response of inelastic solids and structures. The main solution methodology is based on explicit time integration. There are some aspects that make LS-DYNA appropriate to simulate the FSW process. ALE finite element formulations have been implemented into LS-DYNA, which allow large deformation of the workpiece material. With this method, large strains do not cause problematic element distortions. A contact-impact algorithm permits frictional contact between the workpieces and the welding tool. By a specialization of this algorithm, the interfaces between the shoulder and pin can be rigidly tied to admit variable zoning without the need of mesh transition regions. The spatial discretization of the workpieces and the welding tool is achieved by the use of eight-node solid elements with one-point integration and rigid bodies. One-point integration is advantageous due to savings on computer time and robustness in cases of large deformations. Moreover, more than twenty element formulations are available for eight-node solid element. Among 
them, 1 point ALE multi-material element formulation and the constant stress element formulation are applied to the workpieces and the welding tool, respectively. In addition, LS-DYNA currently contains approximately one-hundred constitutive models and ten equations-of-state to cover a wide range of material behavior.

\subsection{Explicit Time Integration and Stability}

Explicit time integration is the numerical method used to solve the time derivatives in the semi-discrete equations (3-37), (3-38) and (3-39). The term "explicit" refers to the fact that the state at the end of the time step is based solely on the state at the beginning of the time step. By the use of this method, the solutions to the above equations are given as follows.

Lagrangian step:

$$
\begin{array}{ll}
\rho_{n+1}^{L}=\rho_{n}+\left.\frac{\partial \rho_{n}}{\partial t}\right|_{\mathbf{X}} \Delta t \text { with } & \left.\frac{\partial \rho_{n}}{\partial t}\right|_{\mathbf{x}}=-\left(\mathbf{M}_{n}^{\rho}\right)^{-\mathbf{1}} \mathbf{K}_{n}^{\rho} \boldsymbol{\rho}_{n}^{L} \\
\mathbf{v}_{n+1}^{L}=\mathbf{v}_{n}+\left.\frac{\partial \mathbf{v}_{n}}{\partial t}\right|_{\mathbf{x}} \Delta t \text { with } & \left.\frac{\partial \mathbf{v}_{n}}{\partial t}\right|_{\mathbf{x}}=\mathbf{M}_{n}^{-1}\left(\mathbf{f}_{n}^{\text {ext }}-\mathbf{f}_{n}^{\text {int }}\right) \\
\sigma_{n+1}^{L}=\sigma_{n}+\left.\frac{\partial \sigma_{n}}{\partial t}\right|_{\mathbf{x}} \Delta t \text { with } & \left.\frac{\partial \sigma_{n}}{\partial t}\right|_{\mathbf{x}}=-\left(\mathbf{M}_{n}^{\sigma}\right)^{-\mathbf{1}} \mathbf{z}_{\mathbf{n}}
\end{array}
$$

Advection step:

$$
\begin{aligned}
& \rho_{n+1}=\rho_{n+1}^{L}+\left.\frac{\partial \rho_{n+1}^{L}}{\partial t}\right|_{\chi} \Delta t \\
& \mathbf{v}_{n+1}=\mathbf{v}_{n+1}^{L}+\left.\frac{\partial \mathbf{v}_{n+1}^{L}}{\partial t}\right|_{\chi} \Delta t \\
& \sigma_{n+1}=\sigma_{n+1}^{L}+\left.\frac{\partial \sigma_{n+1}^{L}}{\partial t}\right|_{\chi} \Delta t
\end{aligned}
$$


Explicit method is computational fast but is conditionally stable. The time step size $\Delta t$ must be less than a critical value $\Delta t_{c r i t}$ otherwise computational errors will grow resulting in a bad solution. A time step size during the solution is determined by

$$
\Delta t=\alpha \cdot \Delta t_{c r i t}
$$

where $\alpha$ is a reduction factor, for stability reason, which is typically set to a value of 0.90 (default in LS-DYNA) or some smaller value; $\Delta t_{\text {crit }}$ is the length of time it takes a signal traveling at the speed of sound in the material to transverse the distance between the node points. $\Delta t_{c r i t}$ for this problem can be calculated by

$$
\begin{aligned}
& \Delta t_{c r i t}=\frac{2}{\omega_{\max }} \leq \min _{e, I} \frac{2}{\omega_{I}^{e}}=\min _{e} \frac{L_{e}}{c_{e}} \\
& L_{e}=\frac{v_{e}}{A_{e \max }}
\end{aligned}
$$

where $\omega_{\max }$ is the largest natural circular frequency, $c_{e}$ is the sound speed in element $e$, $L_{e}$ is a characteristic length of element $e, v_{e}$ is the element volume, $A_{e \max }$ is the area of the largest side.

\subsection{Problem Definition}

In this work, FSW simulations are performed on the butt friction stir weld of the 6061-T6 aluminum alloy workpieces. The welding tool (i.e. the pin and shoulder) is made of steel. The problem is defined in Fig.4-1. Only a limited part of the workpieces is included. The two workpieces with a semicircular section have a diameter of 3.0 in and a thickness of $0.125 \mathrm{in}$. The pin is modeled as a rigid cylinder with a diameter of $0.2552 \mathrm{in}$ and a thickness of $0.12 \mathrm{in}$. The shoulder is also modeled as a rigid cylinder of diameter 
0.7656 in and thickness $0.05 \mathrm{in}$. The pin and shoulder are rigidly constrained together with a rotational velocity of $94.2 \mathrm{rad} / \mathrm{sec}(900 \mathrm{rpm})$ and move relative to the workpieces at a velocity of $0.2 \mathrm{in} / \mathrm{sec}$. Note that the welding tool moves from down to up along the joint line and rotates in the counterclockwise direction in the model. The forging force of the welding tool on the workpieces is given by a displacement of 0.005 in along the depth of the weld.

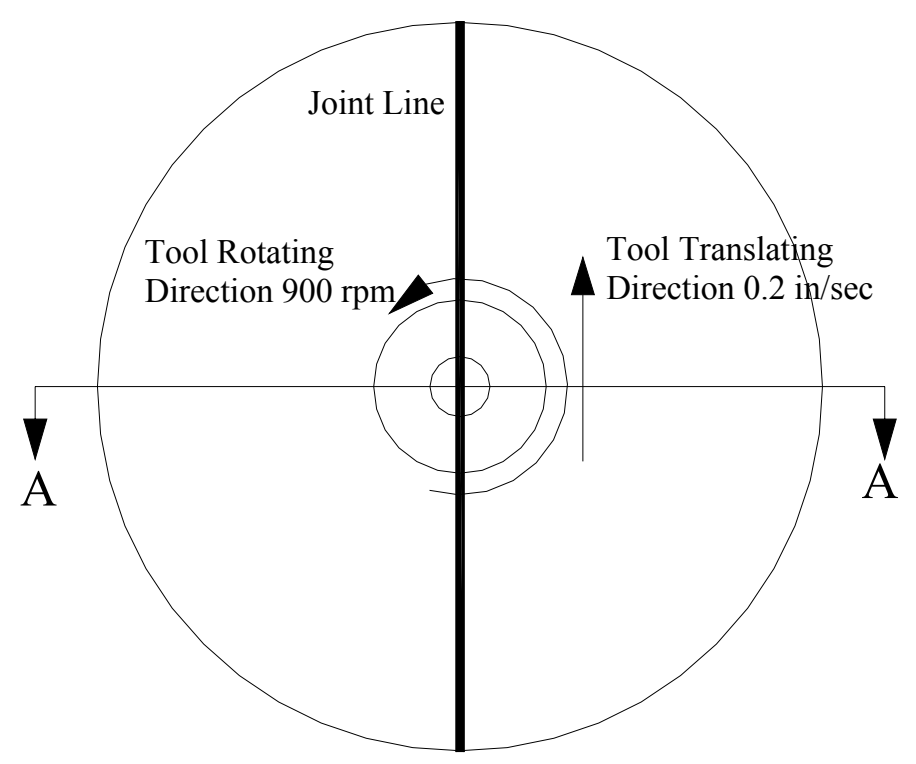

\section{A-A Section}

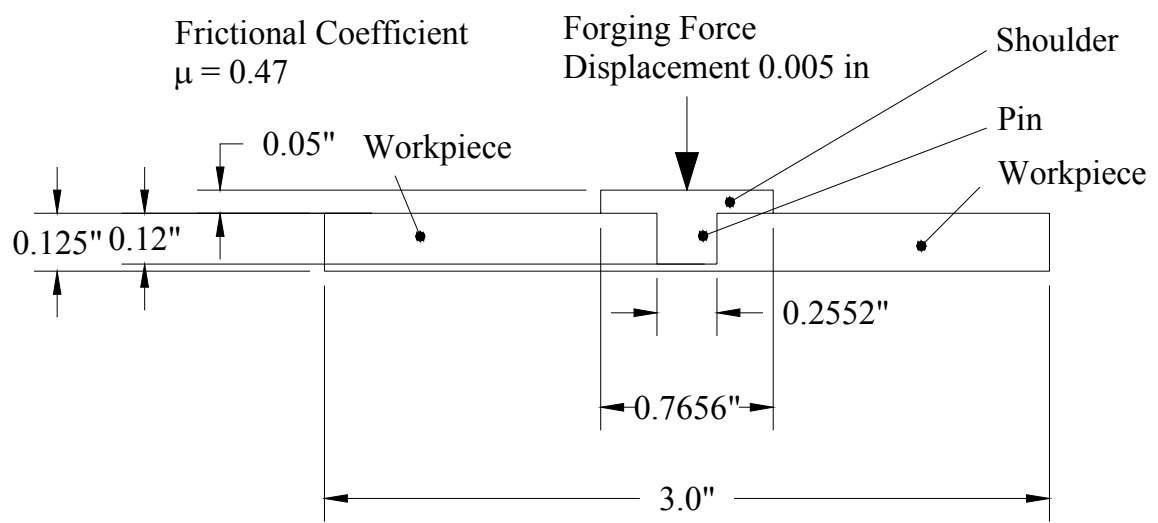

Figure 4-1 Problem Definition 


\subsection{Assumptions}

The simulation procedure employed in this research is focused on determining the deformation and material flow during FSW, so the following assumptions are made in the models:

- The workpiece material is assumed elastic-plastic.

- The strain-rate and temperature effects on the material properties are ignored in the analysis. A fully coupled thermomechanical simulation is not impractical at this time due to the current PC-based computer power.

- The pin and shoulder are modeled as rigid cylinders since they are made of a material of heat treated steel 4140 much stiffer than the workpiece material of aluminum alloy 6061-T6.

- The interface between the workpieces and tool is frictional contact with a frictional coefficient of $\mu$.

- The pin and shoulder are rigidly constrained together.

- To facilitate a more rapid execution of the finite element simulations, tool rotational and relative tool-workpiece translational velocities are both increased 100 times actual values in the analysis. Since the workpiece material model is of the rate independent type, the change in velocities will not have a significant effect on the simulation results. 


\subsection{Finite element discretization}

Eight-node hexahedron solid elements with one integration point plus hourglassing control are used to discretize the two workpieces, the pin and shoulder. One-point integration solid elements are advantageous due to substantial savings on computer time and robustness in cases of large deformations compared to fully-integrated solid elements. However, the biggest disadvantage to one-point integration solid elements is that they are prone to zero energy modes which are also called hourglassing modes (see Flanagan et al. [17], Hallquist [26], and LS-DYNA User's Manual [40]). These modes are oscillatory in nature and tend to have periods that are much shorter than those of the structural response (i.e. they result in mathematical states that are not physically possible). They typically have no stiffness and give a zigzag appearance to a mesh known as hourglass deformations. The occurrence of hourglass deformations in an analysis can invalidate the results and should always be minimized. One way of resisting undesirable hourglassing is with a viscous damping or small elastic stiffness capable of stopping the formation of the anomalous modes but having a negligible effect on the stable global modes. As a general guideline to determine whether hourglassing effects have significantly degraded the results, the hourglassing energy should not exceed $10 \%$ of the internal energy.

Fig.4-2 shows the finite element discretization of the two workpieces, the pin and shoulder. In the Figure, the blue and red shades represent the two workpieces. The yellow and green shades are the shoulder and pin, respectively. 3600 solid elements discretize two workpieces and there are 570 and 340 solid elements in the pin and shoulder, respectively. Totally, 6642 element nodes are used in the entire model. Fig.4-2(top) 
shows the entire model (the pin is beneath the shoulder). Fig.4-2(middle) shows the pin, shoulder and one workpiece. The other workpiece is not shown up in order to display the pin. Fig.4-2(bottom) shows the detail of the zone around the welding tool.

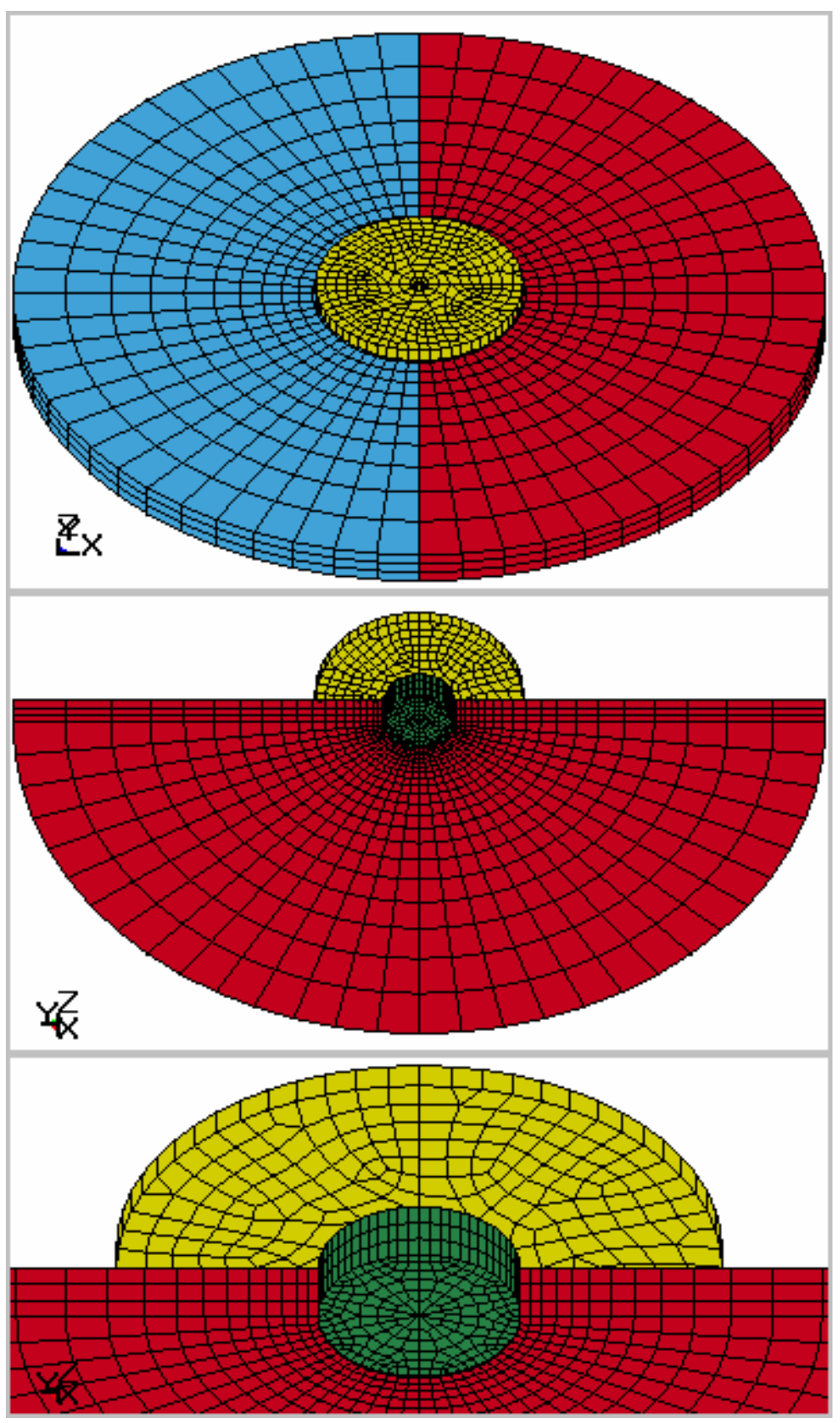

Figure 4-2 Finite Element Discretization of the Two Workpieces (Blue and Red), the Pin (Green) and Shoulder (Yellow) 


\subsection{Material Model}

The chosen materials for the two workpieces, the pin and shoulder are given in Table 4-1. Material \#1 is assigned to the two workpieces, which are made of Al 6061-T6. True stress-strain curves of $\mathrm{Al}$ 6061-T6 at various temperatures are shown in Fig.4-3 (Deng et al. [10]). True stress-strain curve shows a linear range that constitutes elastic deformation at a temperature lower than $500^{\circ} \mathrm{F}$. But, the true stress and true stain seem to have a power law relationship as temperature increases. In this work, the two workpieces are considered ductile materials characterized with elasticity, plasticity and a kinematic hardening effect, but the temperature effect is not considered. Fig.4-4(a) shows the bilinear true stress-strain curve for the elastic-plastic model with kinematic hardening. Fig.4-4(b) shows its special case: elastic-perfectly plastic, which is applied to the current models. In these figures, the horizontal axis is true strain and the vertical axis is true stress. $\sigma_{y}$ is yield stress; $E$ is elastic modulus and $E_{t}$ is tangent modulus.

Material \#2 is assigned to the pin and shoulder, which are made of steel. The pin and shoulder are assumed rigid. 


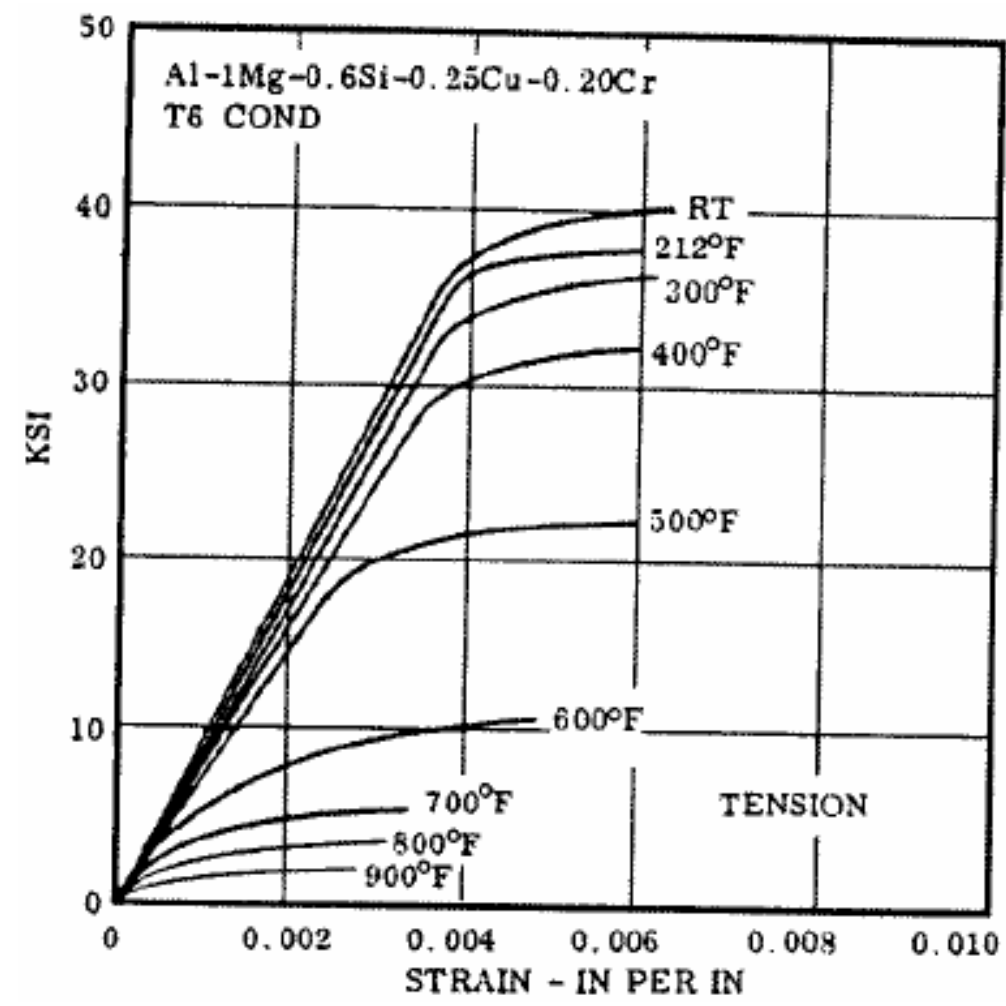

Figure 4-3 True Stress-Strain Curves of Al 6061-T6 at Various Temperatures (Deng et al. [10])

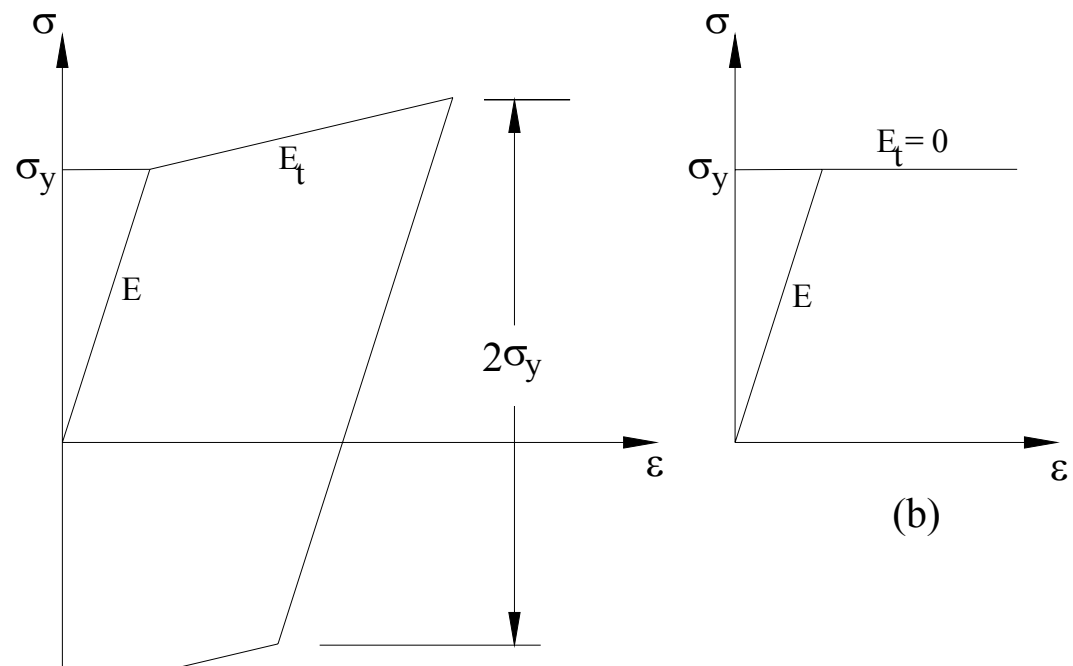

(a)

Figure 4-4 (a) Elastic-plastic Behavior with Kinematic Hardening ( $E_{t}$ is the Slope of the Bilinear True Stress-strain Curve); (b) Elastic-perfectly Plastic Model 


\subsection{Contact Definitions}

The contacts among the workpieces, the pin and shoulder need to be defined before the model is run in LS-DYNA. In order to define the contacts, the two workpieces, the pin and shoulder are assigned to different part No., which are listed in Table 4-2. The contact along the interfaces between the workpieces and welding tool (i.e. pin and shoulder) is assumed as frictional contact with a frictional coefficient of 0.47 (Hallquist [26]), while the pin and shoulder are rigidly tied.

Frictional contact in LS-DYNA is based on a Coulomb formulation. Let $\mathbf{F}_{\text {trial }}$ be the trial force, $\mathbf{F}_{\text {norm }}$ the normal force, $\mathbf{K}$ the interface stiffness, $\mu$ the frictional coefficient, and $\mathbf{F}^{n}$ the frictional force at time $n$. The frictional algorithm presented here uses the equivalent of an elastic-plastic spring and includes the following steps:

1. Compute the yield force $\mathbf{F}_{y}$ :

$$
\mathbf{F}_{y}=\mu\left|\mathbf{F}_{n o r m}\right|
$$

The frictional coefficient $\mu$ used for contact is determined from the static frictional coefficient $\mu_{s}$, the dynamic coefficient $\mu_{d}$, and the exponential decay coefficient $c_{d}$. The frictional coefficient is assumed to be dependent on the relative velocity $\mathbf{v}_{\text {rel }}$ of the surface in contact

$$
\mu=\mu_{d}+\left(\mu_{s}-\mu_{d}\right) e^{-c_{d}\left|\mathbf{v}_{r e l}\right|}
$$

2. Compute the incremental movement of the slave node $\Delta e$

3. Update the interface force to a trial value

$$
\mathbf{F}_{\text {trial }}=\mathbf{F}^{n}-\mathbf{K} \Delta e
$$

4. Check the yield condition 


$$
\begin{array}{llll}
\mathbf{F}^{n+1}=\mathbf{F}_{\text {trial }} & \text { if } & & \left|\mathbf{F}_{\text {trial }}\right| \leq \mathbf{F}_{y} \\
\mathbf{F}^{n+1}=\mathbf{F}_{y} & \text { if } & \left|\mathbf{F}_{\text {trial }}\right|>\mathbf{F}_{y}
\end{array}
$$

The interface shear stress that develops as a result of Coulomb friction can be very large and in some cases may exceed the ability of the material to carry such a stress. Therefore another limit is allowed to be placed on the value of the tangential force. The coefficient for viscous friction $\kappa$ can be used to limit the frictional force to a maximum. A limiting force is computed:

$$
\begin{aligned}
\mathbf{F}_{\text {lim }} & =\kappa \cdot A_{\text {cont }} \\
\mathbf{F}^{n+1} & =\min \left(\mathbf{F}_{\text {coulomb }}^{n+1}, \mathbf{F}_{\text {lim }}\right)
\end{aligned}
$$

where $A_{\text {cont }}$ is the area of the segment contacted by the node in contact. The suggested value for $\kappa$ is to use the yield stress in shear.

$$
\kappa=\frac{\sigma_{0}}{\sqrt{3}}
$$

where $\sigma_{0}$ is the yield stress of the contacted material

In order to avoid undesirable oscillation in contact, a contact damping perpendicular to the contacting surfaces is applied. The contact damping coefficient is calculated as follows

$$
\xi=\frac{c_{v d}}{100} \cdot \xi_{c r i t}
$$

where $c_{v d}$ is the viscous damping coefficient in terms of a percentage(\%) of the critical damping $\xi_{\text {crit }}$ which is determined in the following fashion by LS-DYNA:

$$
\xi_{\text {crit }}=2 m \omega
$$


$\begin{aligned} m & =\min \left(m_{\text {slave, }} m_{\text {master }}\right) \\ \text { where } \quad & w=\sqrt{\mathbf{K} \cdot \frac{m_{\text {slave }}+m_{\text {master }}}{m_{\text {slave }} \cdot m_{\text {master }}}}\end{aligned}$

The pin and shoulder are rigidly tied. Tied contact actually "glues" the slave surfaces to the master surfaces and permits the sudden transitions in zoning. This feature can often decrease the amount of effort required to generate meshes since it reduces the need to match nodes across interfaces of merged parts. The effect of tied contact is that the master surfaces can deform and the slave nodes are forced to follow that deformation.

Penalty method is applied to handling the frictional contact and tie contact along the workpiece/ pin, workpiece/shoulder and pin/shoulder interfaces in this study. Interfaces are defined in three dimensions by listing in arbitrary order all triangular and quadrilateral segments that compromise each side of the interface. One side of the interface is designated as the slave side, and the other is designated as the mater side. Nodes lying in those surfaces are referred as slave and master nodes, respectively. The penalty method consists of placing normal interface springs between all penetrating nodes and the contact surface. The method is found to excite little if any mesh hourglassing. This lack of noise is undoubtedly attributable to the symmetry of the approach. Momentum is exactly conserved without the necessity of imposing impact and release conditions. Furthermore, no special treatment of intersecting interfaces is required, greatly simplified the implementation. The interface stiffness is chosen to be approximately the same order of magnitude as the stiffness of the interface element normal to the interface. Consequently the computed time step size is unaffected by the existence of the interfaces. However, if interface pressures become large, unacceptable 
penetration may occur. By scaling up the stiffness and scaling down the time step size, such problems may still be solved using the penalty method.

\subsection{Loading and Boundary Conditions}

Loading procedure is that (1) the forging force load is applied as a ramp in a very short time to the welding tool, and then this value is kept until termination time. Meanwhile, (2) rotational and translational velocities are applied to the welding tool.

Since the far sides on the workpieces are not affected by the tool/workpiece interface, all the nodes on the circular edge of the workpieces are fixed along $\mathrm{x}$ (transverse), y (longitudinal), z (depth-wise) directions. The bottom of the workpieces is

fixed along $\mathrm{z}$ direction because the two workpieces are supported by a rigidly backing plate during FSW. 
Table 4-1 Material Information

\begin{tabular}{|c|c|c|c|c|c|c|}
\hline \multirow{2}{*}{$\begin{array}{c}\text { Material } \\
\text { No. }\end{array}$} & \multirow{2}{*}{ Material } & \multirow{2}{*}{$\begin{array}{l}\text { Material } \\
\text { Model }\end{array}$} & \multicolumn{4}{|c|}{ Mechanical Properties of Material } \\
\hline & & & $\begin{array}{c}\text { Mass Density } \\
\left(\left(\mathrm{lb}^{*} \mathrm{~s}^{\wedge} 2\right) / \mathrm{in}^{\wedge}\right. \\
4) \\
\end{array}$ & $\begin{array}{c}\text { Young's } \\
\text { Modulus } E \\
\text { (psi) } \\
\end{array}$ & $\begin{array}{l}\text { Poisson } \\
\text { Ratio }\end{array}$ & $\begin{array}{c}\text { Yield Stress } \\
\sigma_{y}(\mathrm{psi})\end{array}$ \\
\hline$\# 1$ & $\begin{array}{l}\text { Workpieces } \\
\text { (A1 6061-T6) }\end{array}$ & $\begin{array}{l}\text { Elastic- } \\
\text { perfectly } \\
\text { Plasticity }\end{array}$ & 0.00025 & $1.0 \mathrm{e} 7$ & 0.33 & 39900 \\
\hline$\# 2$ & $\begin{array}{l}\text { Pin and } \\
\text { Shoulder } \\
\text { (Steel) }\end{array}$ & Rigid & 0.00073 & $3.0 \mathrm{e} 7$ & 0.28 & ---- \\
\hline
\end{tabular}

Table 4-2 Contact Definition

\begin{tabular}{c|c|c|c}
\hline Part No. & Part Name & Material No. & Contact Definition \\
\hline 1 & Half workpiece & $\# 1$ & Frictional contact with part 3, 4 $(\mu=0.47)$ \\
\hline 2 & Half workpiece & $\# 1$ & Frictional contact with part 3, 4 $(\mu=0.47)$ \\
\hline 3 & Pin & $\# 2$ & $\begin{array}{c}\text { Frictional contact with part } 1,2 ; \text { rigidly tied } \\
\text { with part 4 }\end{array}$ \\
\hline 4 & Shoulder & $\# 2$ & $\begin{array}{c}\text { Frictional contact with part } 1,2 ; \text { rigidly tied } \\
\text { with part 3 }\end{array}$ \\
\hline
\end{tabular}




\subsection{Simulation Results}

Before the Lagrangian and ALE computation results are presented, a convention is needed to distinguish between the two sides of a friction stir weld since it is asymmetric along the joint line. First, it is assumed that the relative motion between the tool and the workpieces is due to a moving tool and stationary workpieces. Then, one side of a friction stir weld is defined as "advancing side" where the rotational motion of the tool and its translational motion are in the same direction. The other side, where the tool rotational motion is in the opposite direction to its translational motion, is referred to as "retreating side". Those definitions are indicated in Fig.4-5.

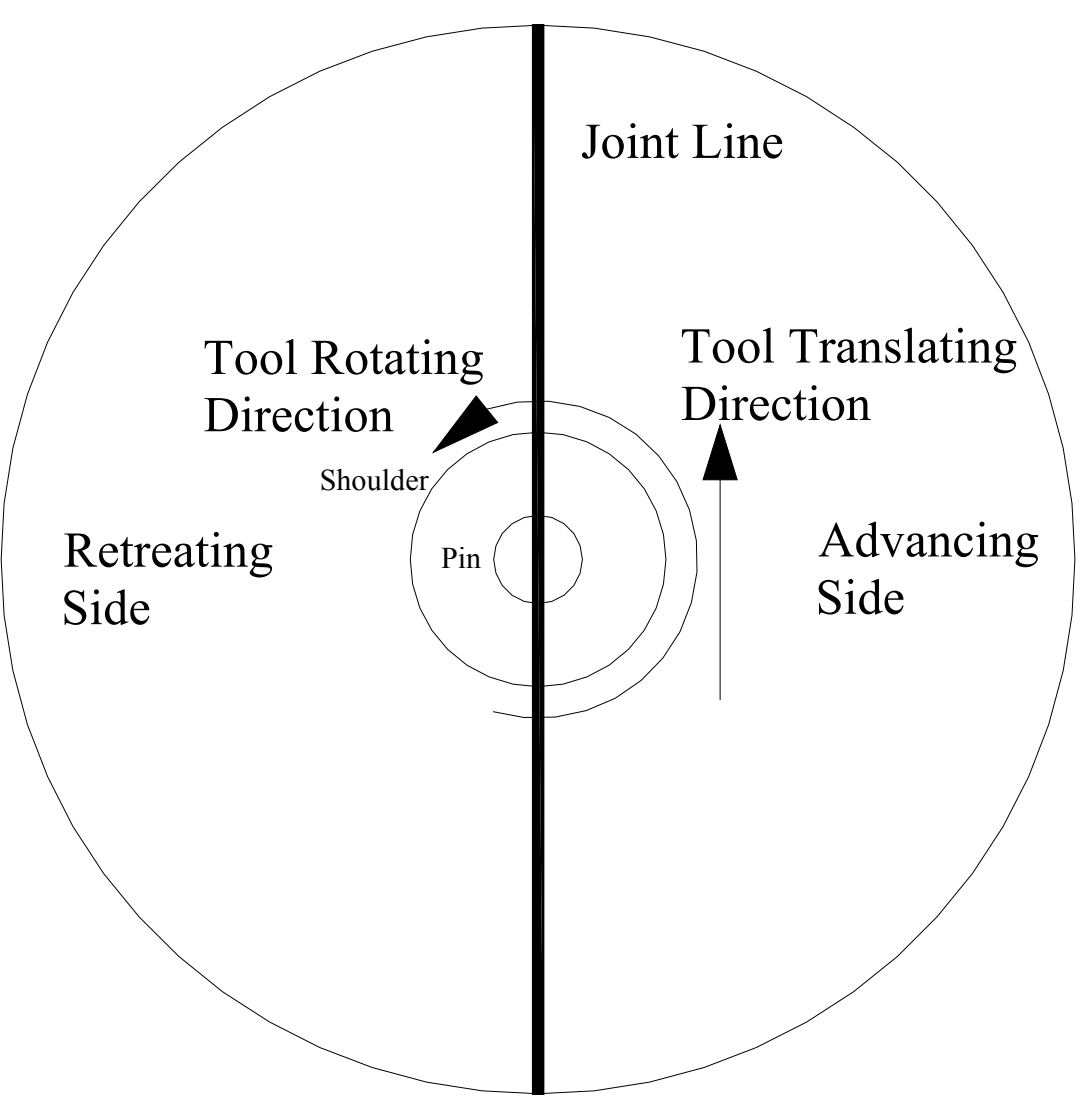

Figure 4-5 Process Terminology 


\subsubsection{Results Based on Lagrangian Formulations}

In the Lagrangian computation, the element nodes are attached to the material points and the elements deform with the material as discussed before. Therefore, the elements can become severely distorted where the large plastic strains appear, which can be observed in the FSW process. The approximation accuracy of the elements then deteriorates. Furthermore, the computations are aborted or severe local inaccuracies are caused.

The plastic strain contours in the workpiece material at $t=0.00005 \mathrm{~s}, 0.0001 \mathrm{~s}$, $0.00015 s$, and $0.0002 s$ are plotted, as shown in Fig.4-6. Fig.4-6 (left) shows the top view of the entire model, and Fig.4-6 (right) is the side view of one workpiece plus the pin and shoulder, in which the detail of the plastic strains in the region around the pin and shoulder is shown. The pin and shoulder are displayed in the green and yellow feature line modes, respectively. In addition, the tool translating and rotating directions, the advancing and retreating sides of the friction stir weld are indicated in the first image.

From the plastic strain contours, it can be observed the large stains of the workpiece material occur at the locations where the workpiece material is in line with the edge of the shoulder. Due to the characteristic of Lagrangian formulations i.e. the Lagrangian elements deform with the material throughout the computation, it can be seen that the distorted elements of the workpieces appear at the same location as the deformed material. Furthermore, it is noticed that as the workpiece material keeps moving, the Lagrangian elements of the workpieces which are in line with the edge of the shoulder get more and more distorted. Because of the large deformations of the finite elements the Lagrangian computation breaks off at about $t=0.0002 \mathrm{~s}$. 

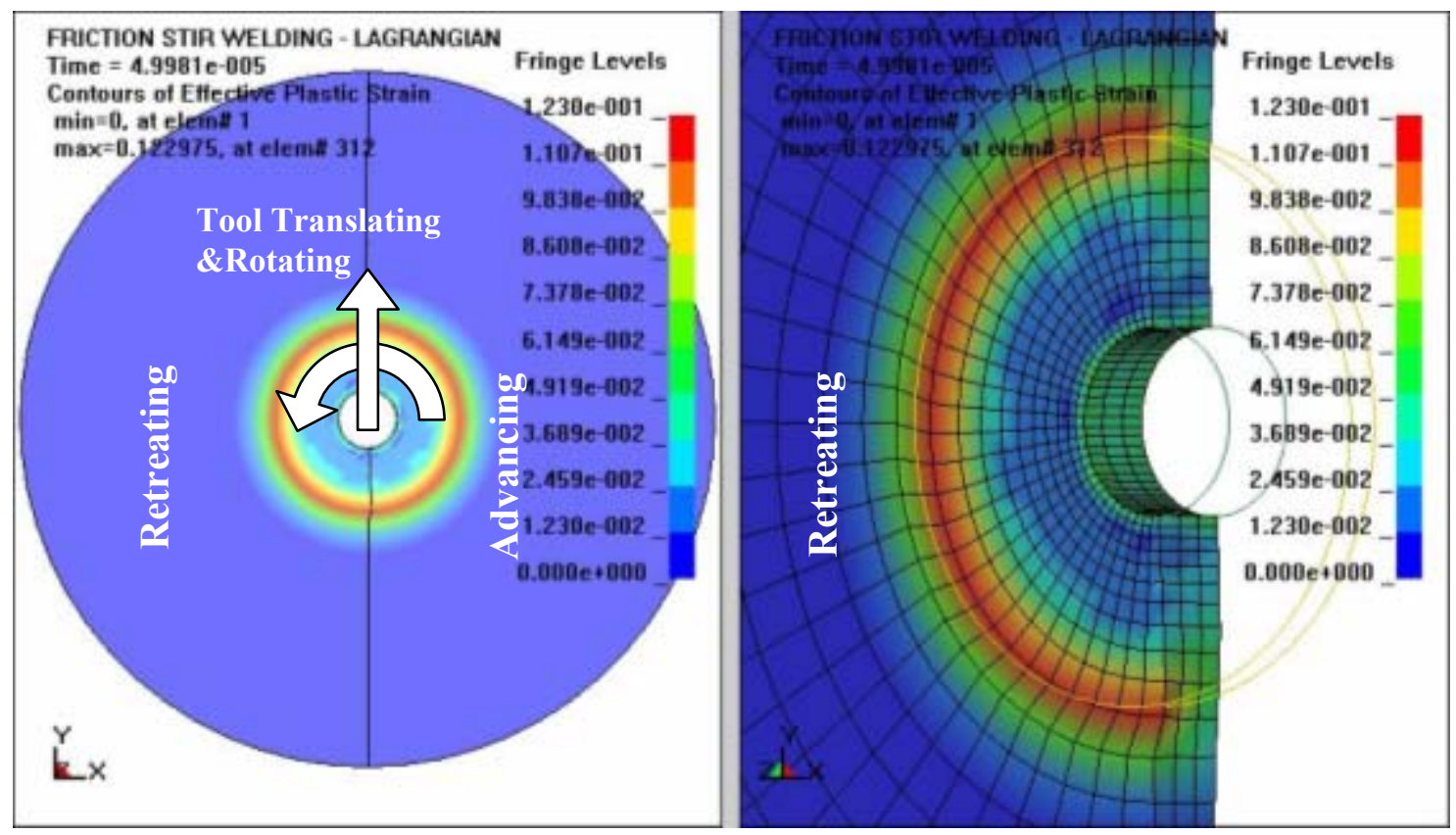

\section{$t=0.00005 \mathrm{~s}$}
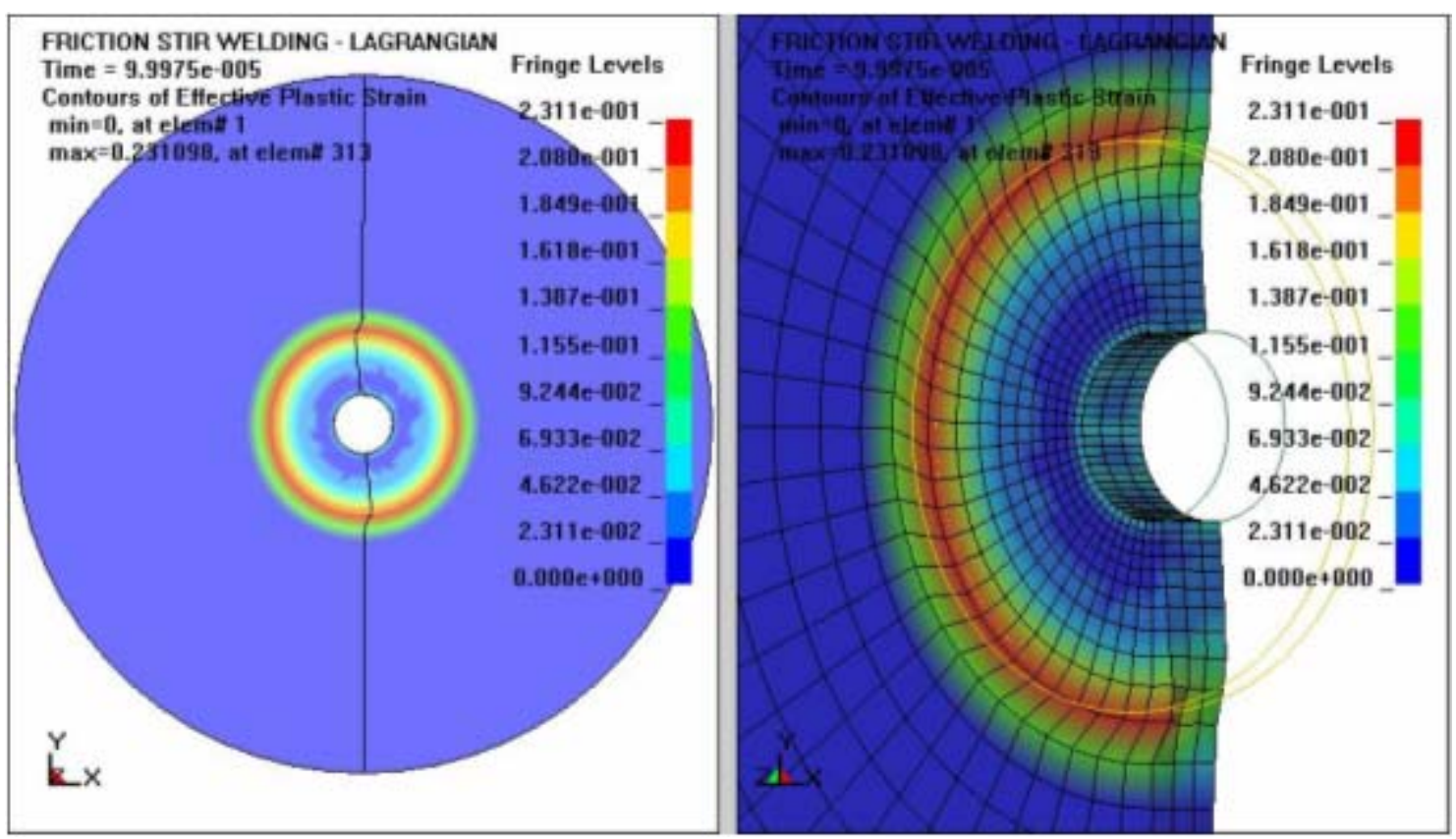

$$
t=0.0001 s
$$



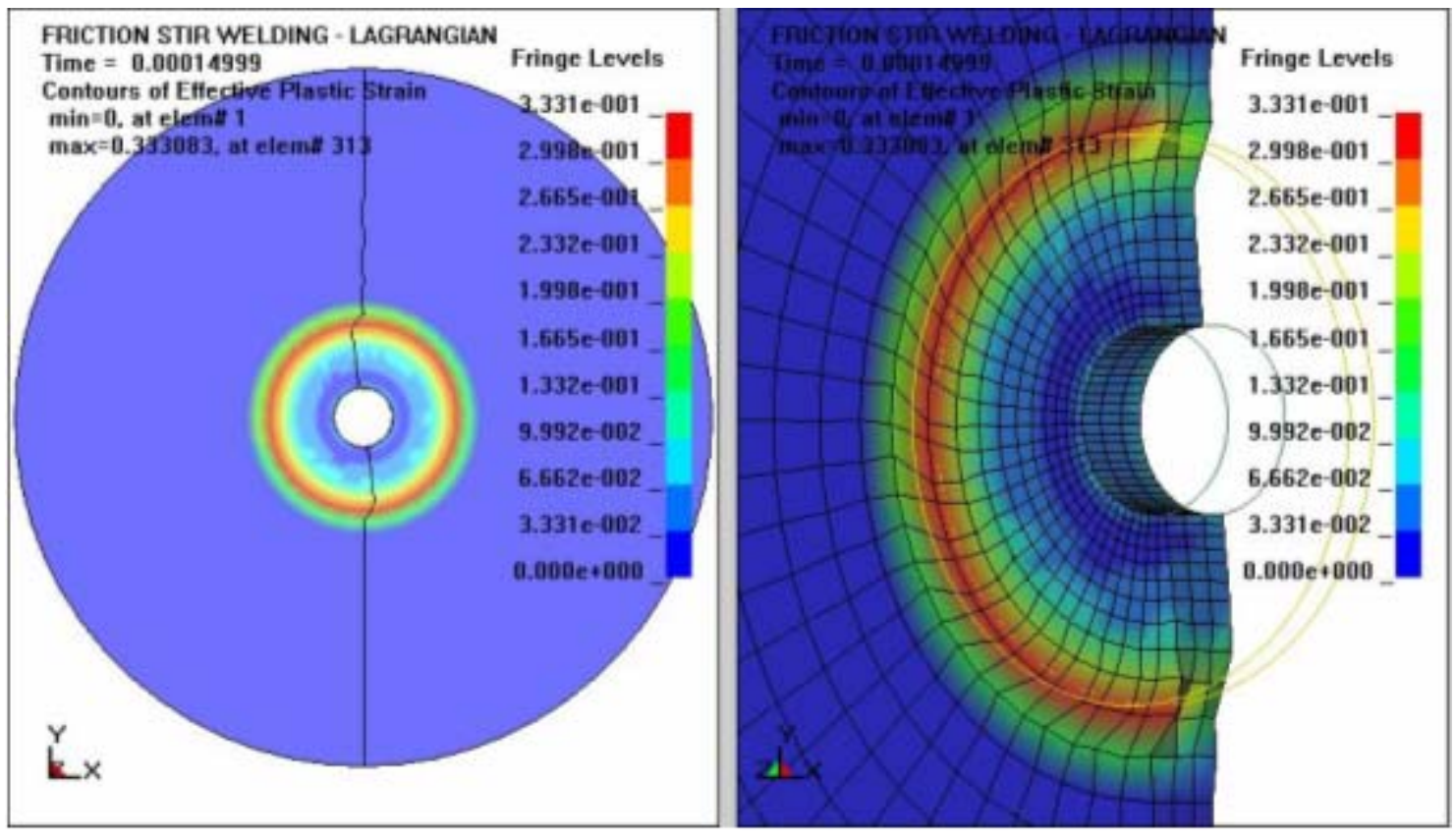

$$
t=0.00015 s
$$
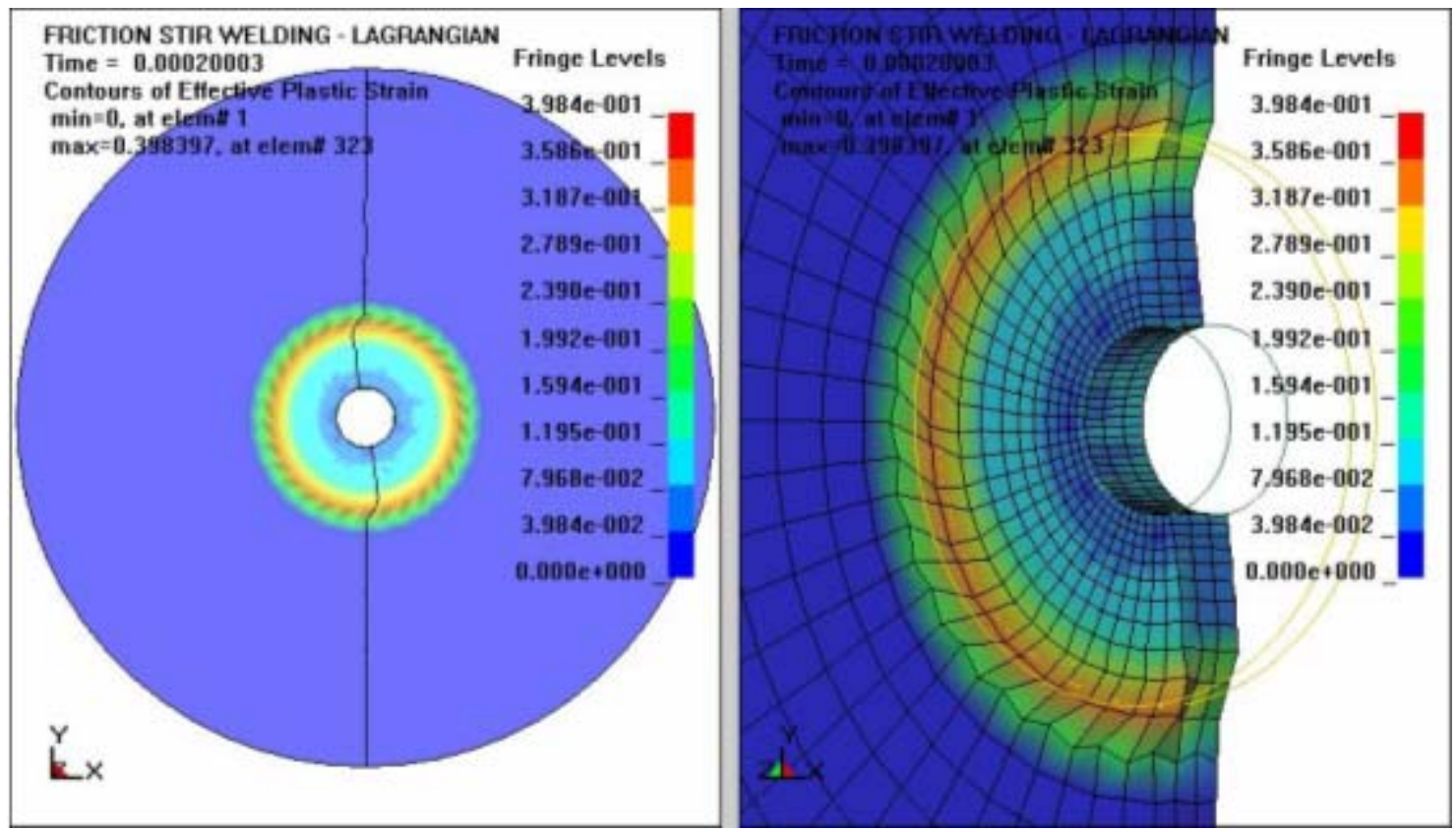

$$
t=0.0002 s
$$

Figure 4-6 Plastic Strains in the Workpieces at Different Time Instances 


\subsubsection{Results Based on ALE Formulations}

According to the computation results based on Lagrangian formulations, it is concluded that Lagrangian formulations are totally inappropriate for such a large deformation problem of FSW. Therefore, ALE formulations are chosen to apply to the simulations of the FSW process. In ALE formulations, as the name suggests, ALE descriptions are arbitrary combinations of the Lagrangian and Eulerian descriptions. The word arbitrary here refers to the fact that the combination is specified by the user through the selection of a mesh motion. Therefore, the ALE mesh does not tangle under intense shear. Of course, a judicious choice of the mesh motion is required if severe mesh distortions are to be eliminated. In this section, the ALE performance on the FSW process is demonstrated.

The effective plastic strain contours for the workpieces at $t=0.025 \mathrm{~s}, t=0.05 \mathrm{~s}, t$ $=0.75 \mathrm{~s}, t=0.1 \mathrm{~s}$ are plotted in Fig.4-7. The tool translating and rotating directions and the definitions of the advancing and retreating sides are indicated in the first image. It is shown that the plastically deforming region is confined to the region immediately surrounding the pin and under the shoulder. The volume outside this plastic region is the elastic region. In addition, the plastic zone in front of the pin is larger than that in the behind of the pin because the contact surfaces between the welding tool and workpieces in front of the pin are larger than the opposite side as the welding tool moves upward along the joint line. A closer inspection on the retreating side shows the plastic strains at the pin/workpiece interface along the circumference of the pin increase from up to down, causing the material in front of the pin to move toward the behind of the pin from the advancing side. 


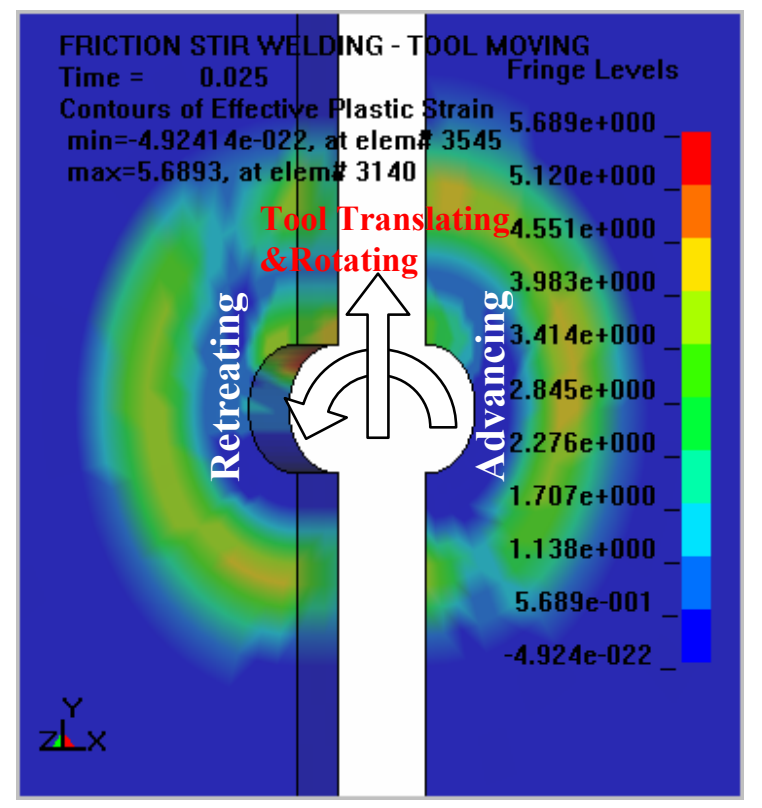

$$
t=0.025 s
$$

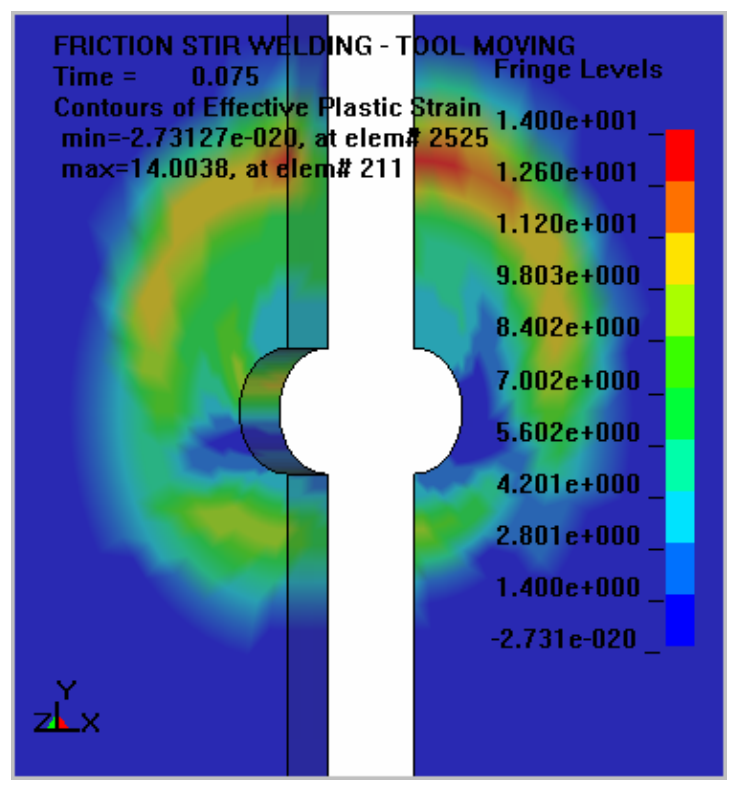

$t=0.075 s$

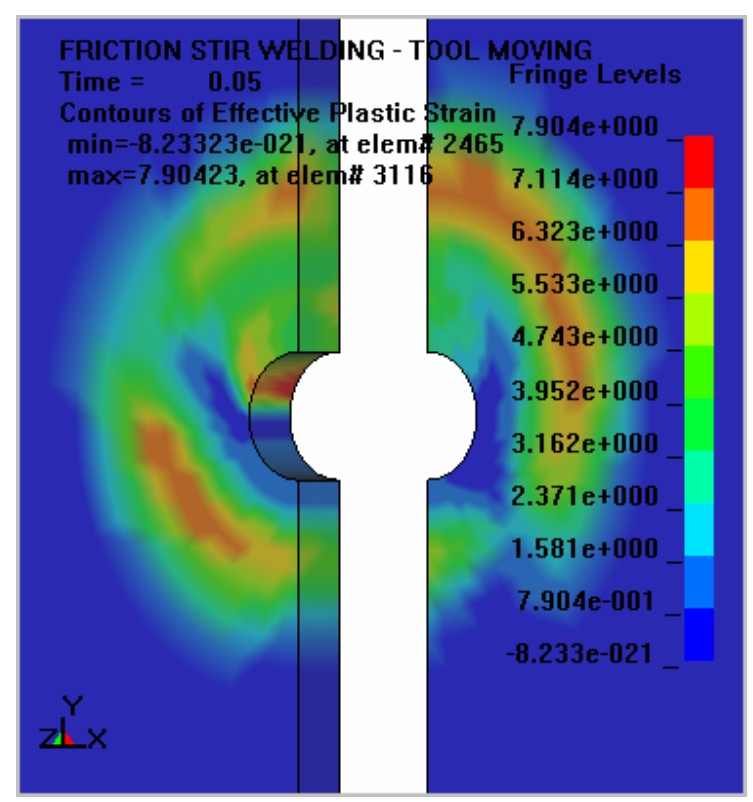

$$
t=0.05 s
$$

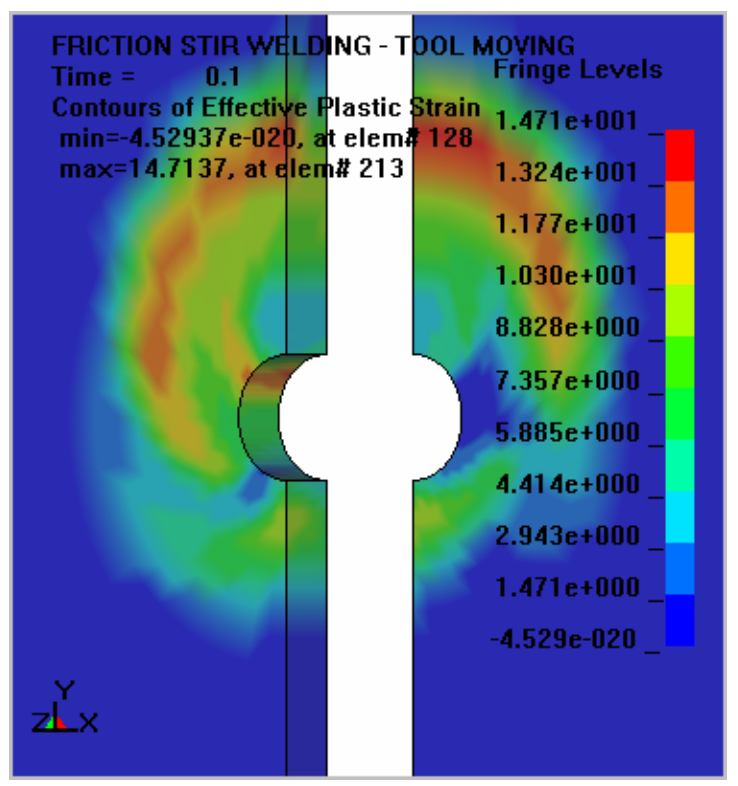

$t=0.1 s$

Figure 4-7 Effective Plastic Strain Contours of the Workpieces at Different Time Instances 
The contours of history variable \#2 for the workpieces at $t=0.025 \mathrm{~s}, 0.050 \mathrm{~s}$, $0.075 s$ and $0.1 s$ are presented in Fig.4-8. The advancing side is on the right and the retreating side is on the left, as shown in the first volume fraction contour. The tool translating and rotating directions are also indicated in the first image. History variable \#2 is defined as the volume fraction of the workpieces. The volume fraction values are usually 0 to 1 which means that full volume fraction for the right half workpiece is 1 and volume fraction for the left half workpiece is 0 . Values between 0 and 1 are partial volume. Fig.4-8 (right) shows the spatial ALE mesh corresponding to each volume fraction contour at the different time instances. The region around the pin and shoulder (green and yellow feature lines) is shown in detail.

The volume fraction contours show that the material flow in not symmetric about the joint line. The flow patterns on the advancing and retreating sides of the friction stir weld are different. By the rotation of the welding tool, the material in the advancing side is transported around the pin to the retreating side in the rotational direction of the tool, and then move to the behind of the pin. The material in the retreating side is also transported around the pin to the advancing side in the rotational direction of the tool, and then move forward on the advancing side along the translational direction of the tool. Although the material around the shoulder and pin deforms dramatically, the finite elements are still well distributed throughout the ALE computation, as can be seen in Fig.4-8 (right), due to the fact ALE formulations smooth out the large mesh distortions.

Additionally, it is noticed that a hole in the middle of the workpieces is left behind the pin as the welding tool proceeds along the joint line. But in reality that hole should be filled with the workpiece materials and only a hole exists at the place where the pin is. To 
resolve this problem, a mesh motion scheme will be developed for the ALE modeling of the FSW process. In the next chapter, the simulations of the FSW process with such a scheme will be discussed.
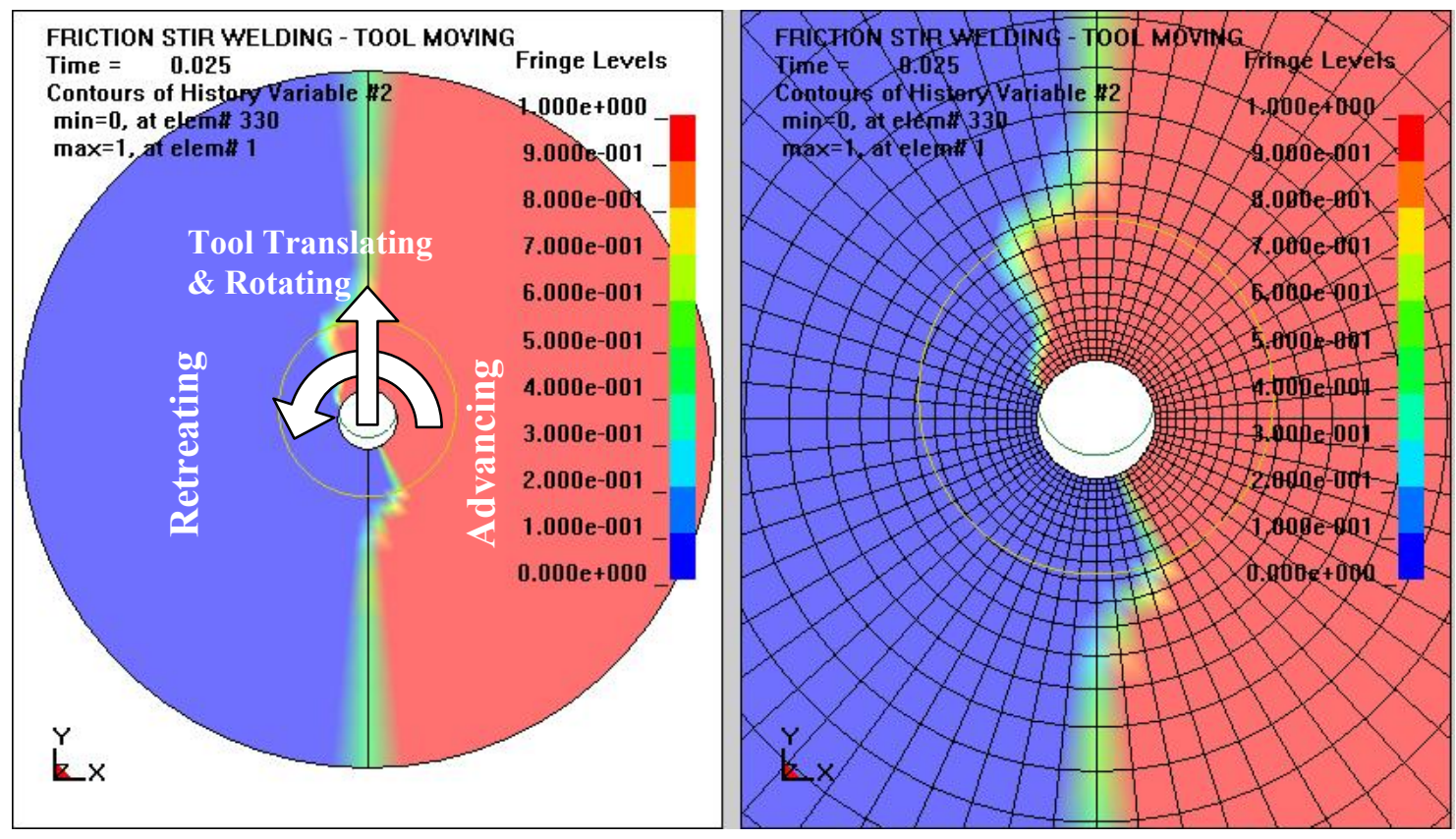

$$
t=0.025 \mathrm{~s}
$$
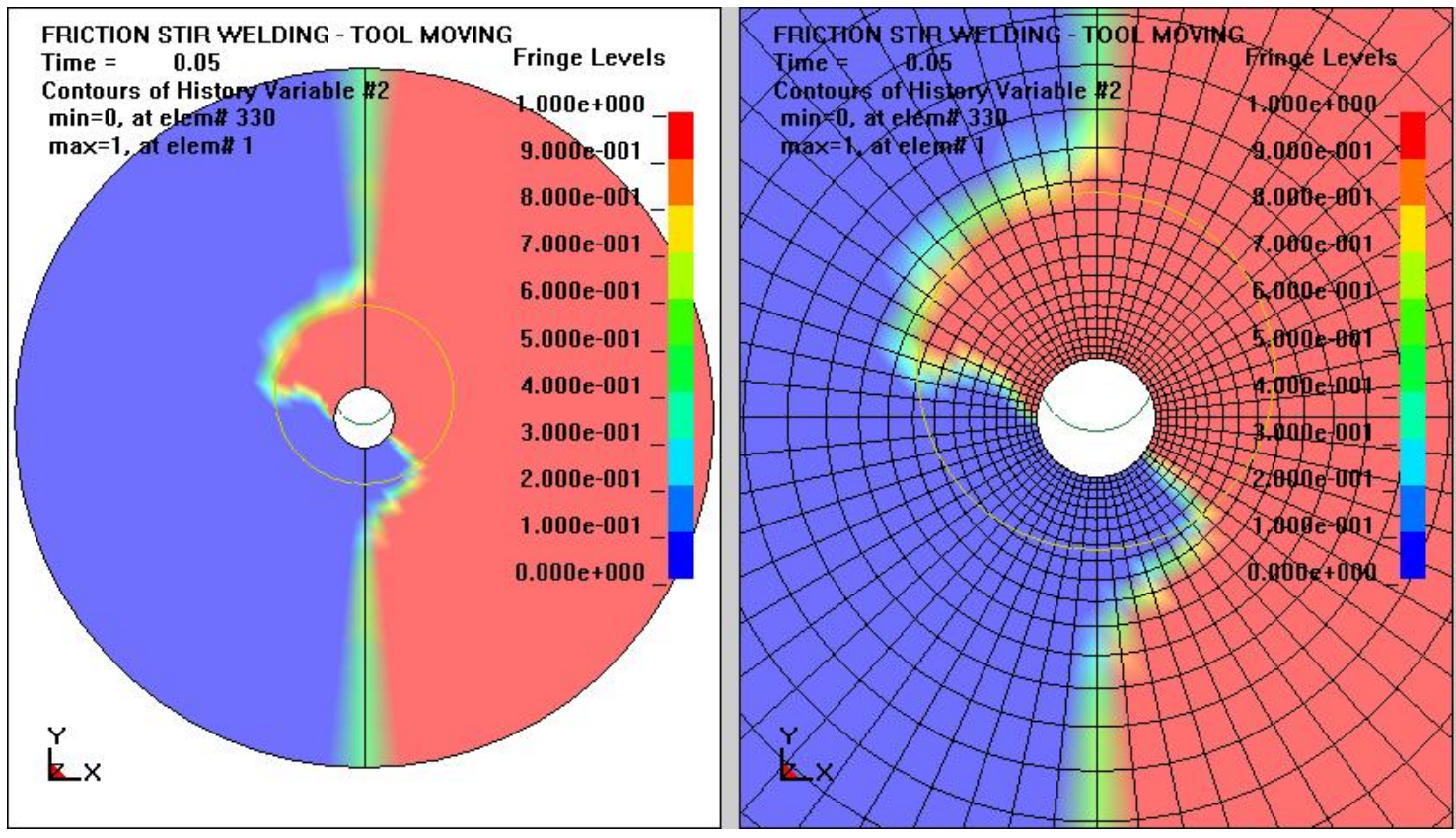

$t=0.050 s$ 

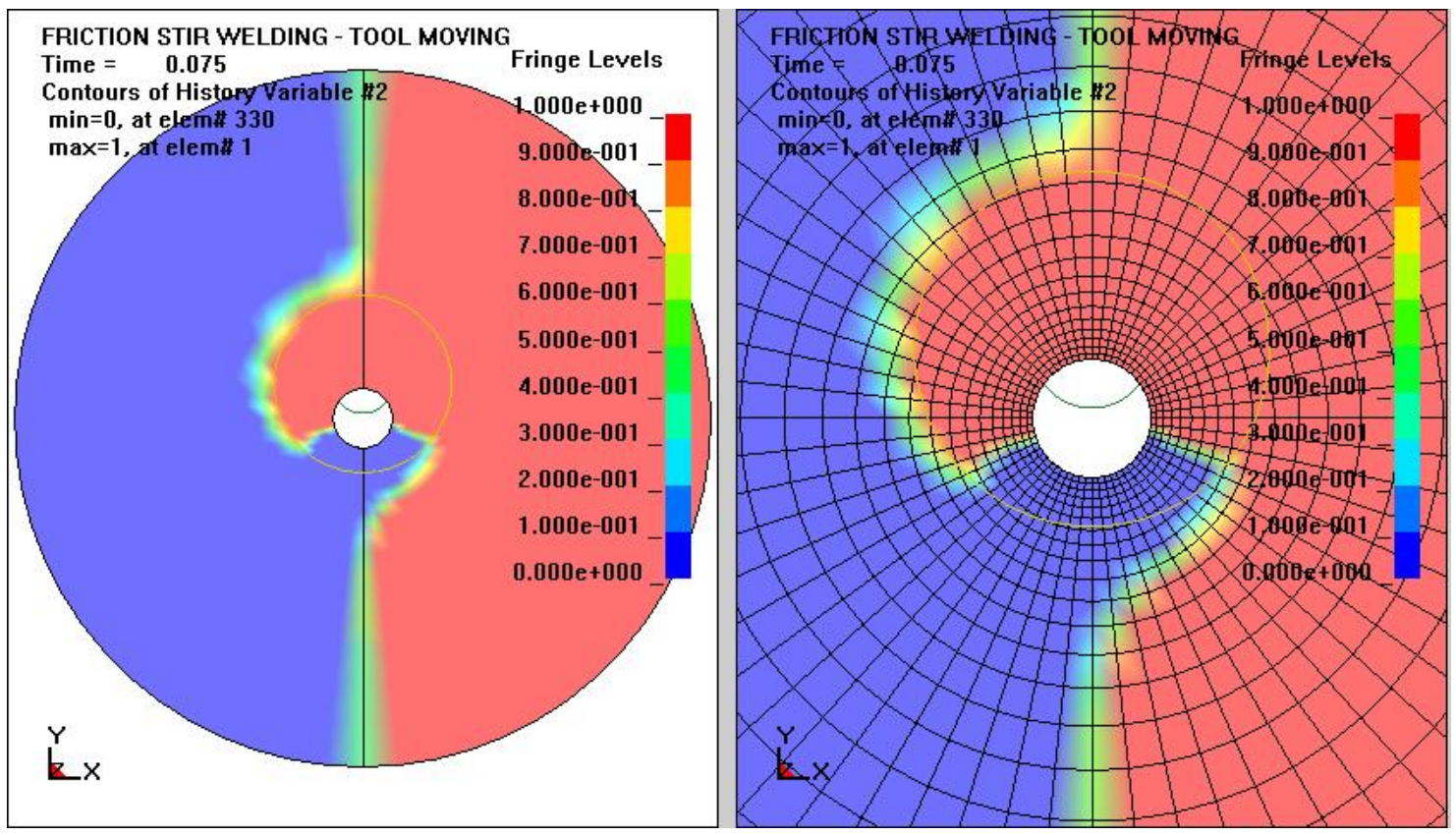

$$
t=0.075 s
$$
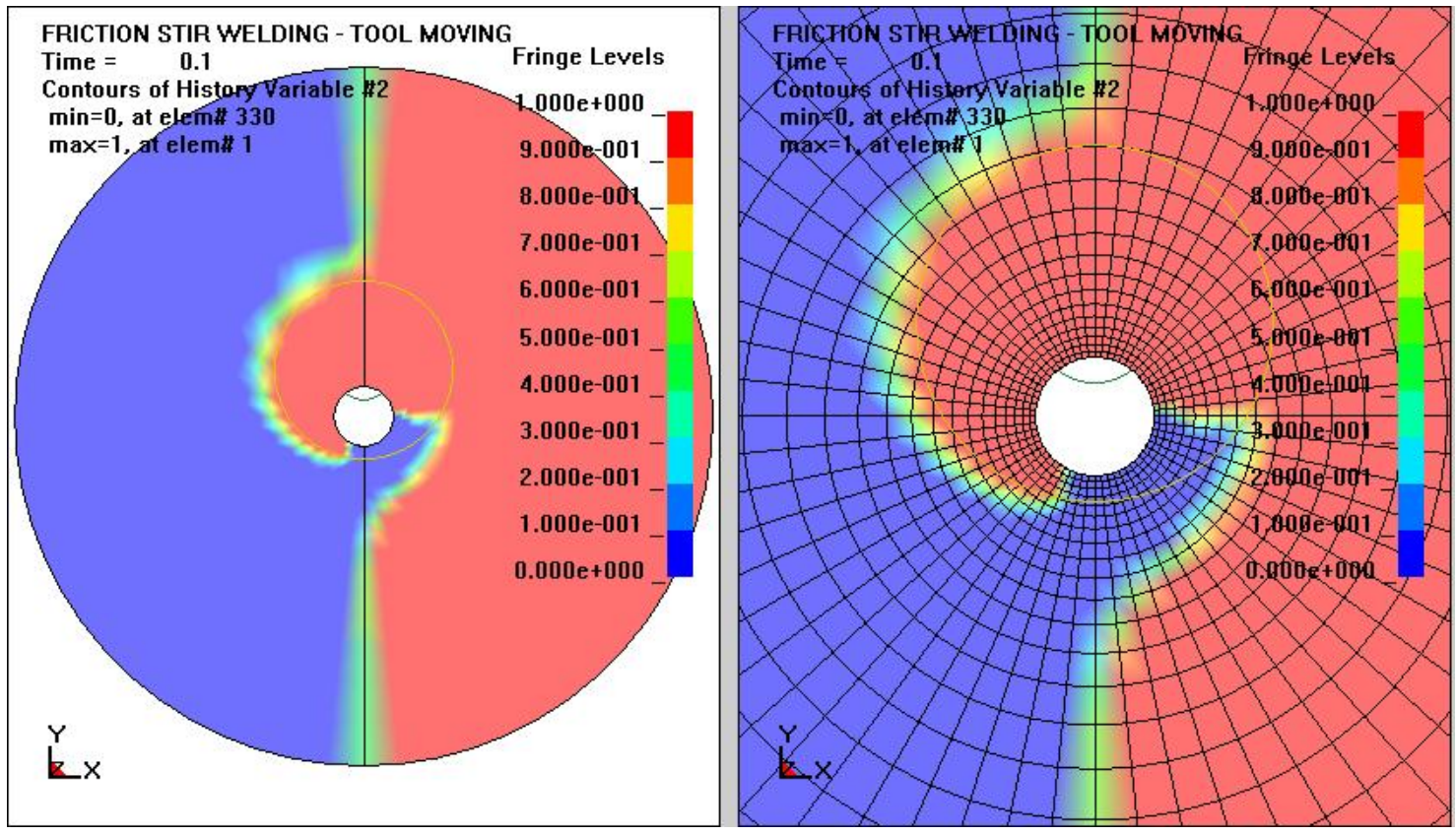

$$
t=0.1 \mathrm{~s}
$$

Figure 4-8 Volume Fraction Contours and Spatial Mesh of the Workpieces at Different Time Instances 
Since the one-point integration elements are used for the simulations, it is always important to determine whether hourglassing effects have significantly degraded the results. The internal energy and hourglass energy over time for two workpieces (designated as "Matsum Data") are plotted in Fig.4-9. The red curve with letter A and green curve with letter B represent the internal energy for part one and two (i.e. advancing side and retreating side), respectively. And the dark and light blue curves with letter $\mathrm{C}$ and $\mathrm{D}$ represent the hourglass energy for each part, respectively. From the figure, it is shown that the internal energy (curve A) inside part one (i.e. advancing side) increase more slowly than that inside part two (i.e. retreating side) that indicates the material plastic deformation in the advancing side is smaller than the retreating side. In addition, by checking the ratio of hourglass energy over internal energy, it is less than $10 \%$ for each part that means the hourglass mode doesn't cause bad effects on the simulation results.

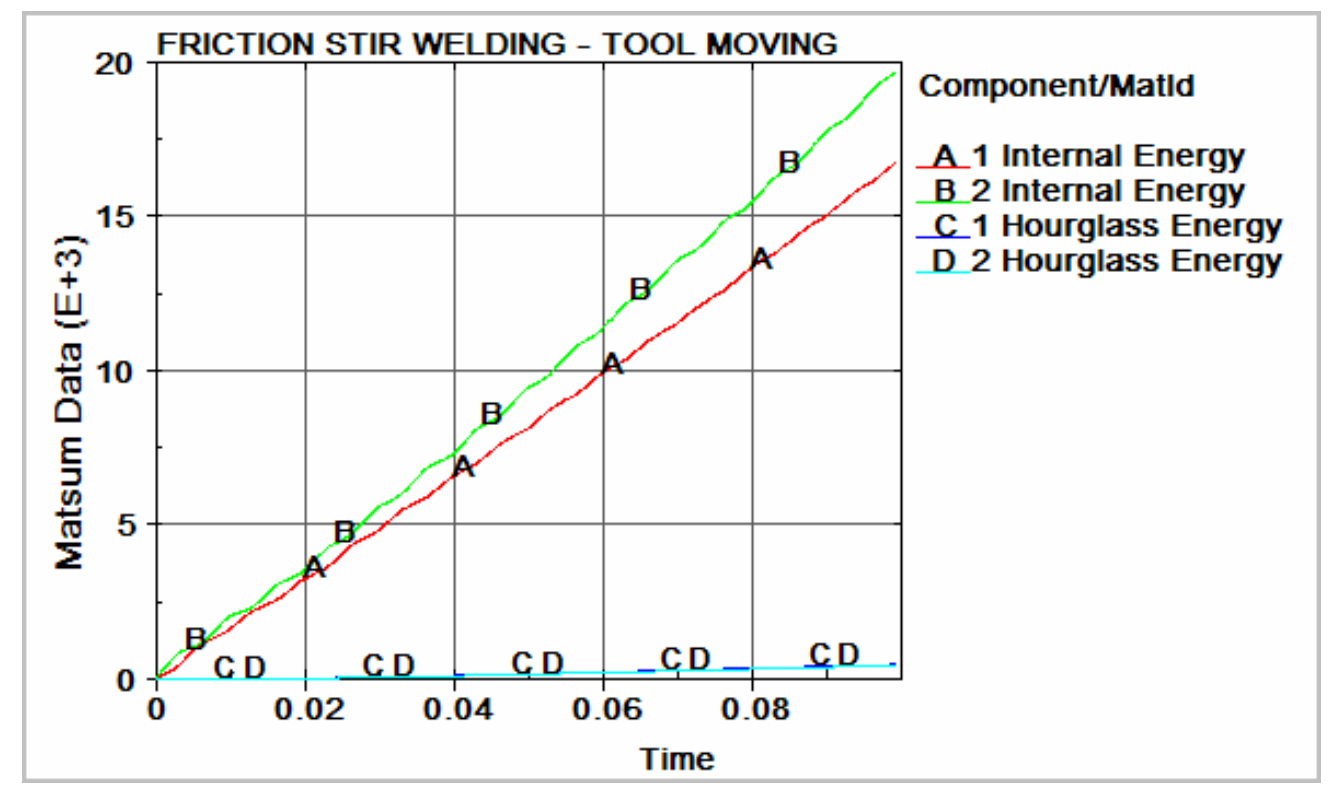

Figure 4-9 Internal and Hourglass Energies vs. Time for the Two Workpieces 


\section{CHAPTER 5 MOVING MESH FOR THE ALE MODELING OF FSW}

\subsection{Moving Mesh}

In the finite element simulations of FSW performed in the previous chapter, the pin and shoulder rotate and translate along the joint line relative to the workpieces, as shown in Fig.5-1. In the figure, the middle region within the blue lines is for the modeling work. The black crosses represent the workpiece material particles. Finite element simulation results show that an unexpected hole indicated with the red circle in Fig.51(b), is left behind the pin instead of right on the location of the pin as the welding tool travels a distance of $d$ along the joint line. In the actual FSW process, this hole should be filled by the workpiece material as the welding tool proceeds.

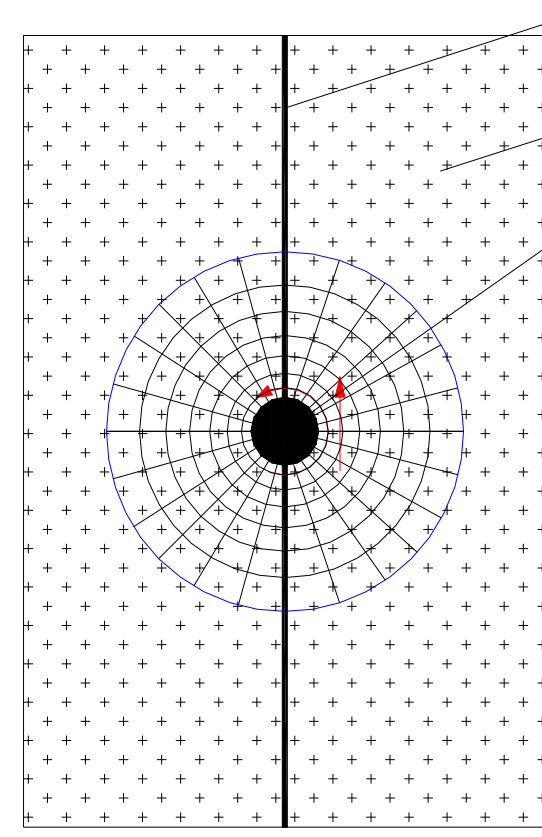

(a)
Joint line

Workpiece

Rotating and translating pin (Shoulder is not Shown)

+ Workpiece Material Particles

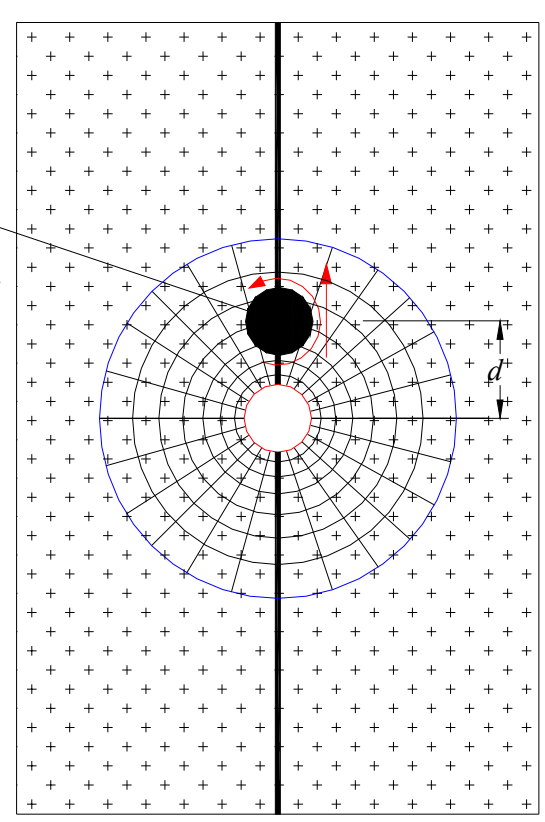

(b)

Figure 5-1 FSW Simulation with a Rotating and Translating Pin: (a) Initial State; (b) Steady State 
The above issue can be resolved by applying a method of moving mesh to the ALE simulations of FSW performed in the previous chapter, and thus it is possible to simulate FSW as a continuous process. The method is illustrated in Fig.5-2. In the figure, the middle region within the blue lines is of interest for the modeling work. ALE formulations are applied to this region. In addition, a shrink region may be put on its top and an expansion region is located at its bottom. These two regions are of Lagrangian nature. The red crosses represent the workpiece material particles in the middle region and the black crosses represent the workpiece material particles in the top and bottom regions.

Let the workpieces move along the joint line in a translational velocity relative to the welding tool, and let the welding tool rotate at a fix position throughout the computation. As the workpiece material moves a distance of $d$ along the joint line, the top region has to contract and the bottom region has to expand, but the finite element mesh in the middle region is still fixed in space while the material (right crosses) flows through this region. Fig.5-2 (b) shows the steady state of the finite element model after the workpiece moves downward a distance of $d$ along the joint line. Therefore, the above method can be used to simulate the FSW process in a continuous manner. 


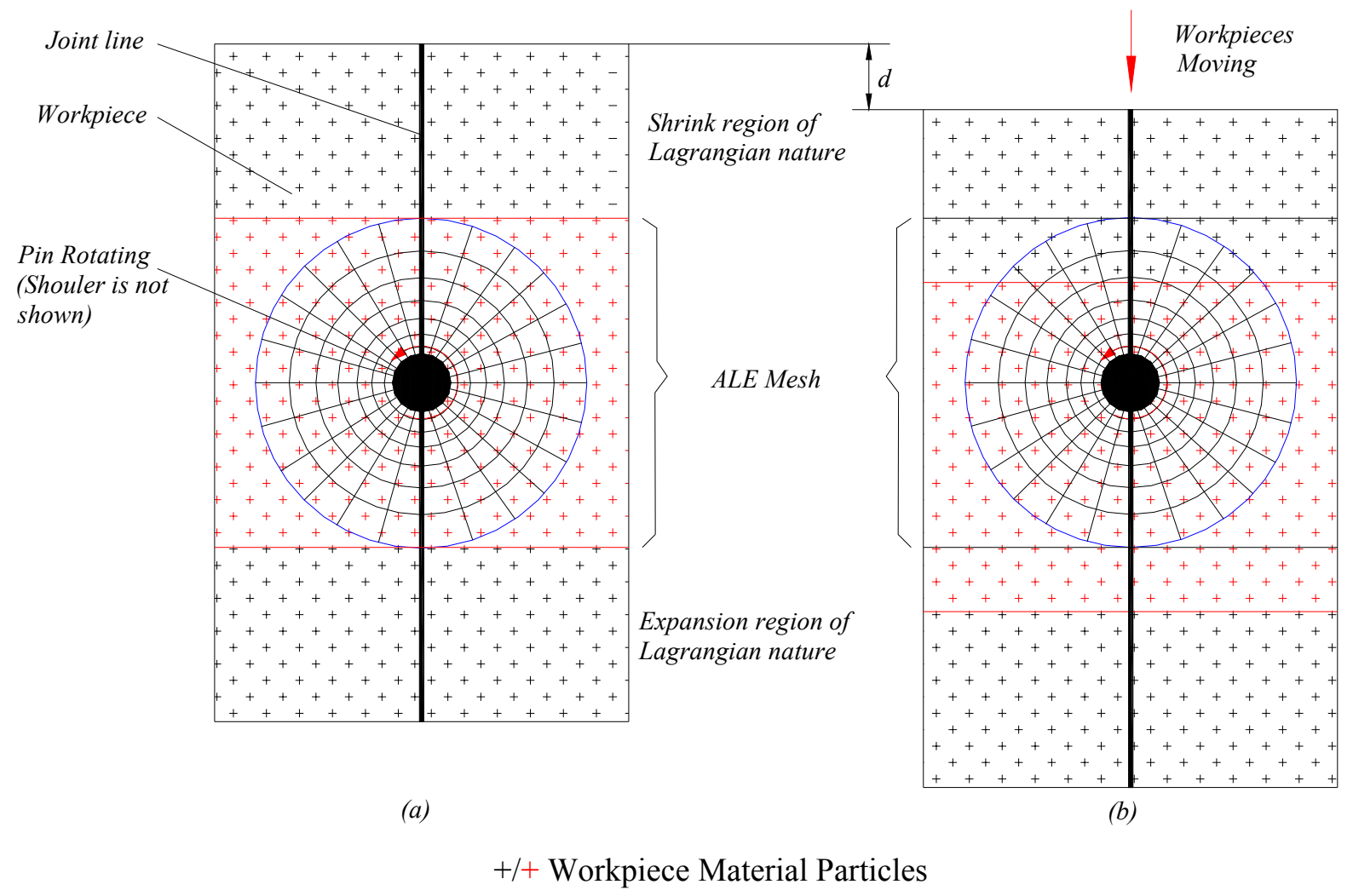

Figure 5-2 FSW Simulation Using the Moving Mesh Method: (a) Initial State; (b) Steady State

In nature, the mesh motion in the middle region shown in Fig.5-2 involves two steps in the context of ALE formulations: Lagrangian step and advection step. Fig.5-3 illustrates the mesh motion in those two steps. For simplicity, one element is taken out from the middle region in Fig.5-2 as an example. In the figure, the rectangle with the solid lines is the original element with node $i, j, k, 1$. During the Lagrangian step, the element nodes move with material deformation. The rectangle with the red dashed lines shows the deformed element in such a step. Total deformation displacement $\delta$ of each node consists of two parts: $\delta y$ and $\delta r$ in the Lagrangian step. Workpiece translation produces a displacement of $\delta y$ and tool rotation yields a displacement of $\delta r$. Following 
the Lagrangian step, the advection step is performed. The distorted element caused in the Lagrangian step is moved back to its original location during the step. Then the Lagrangian solutions are mapped onto the new mesh.

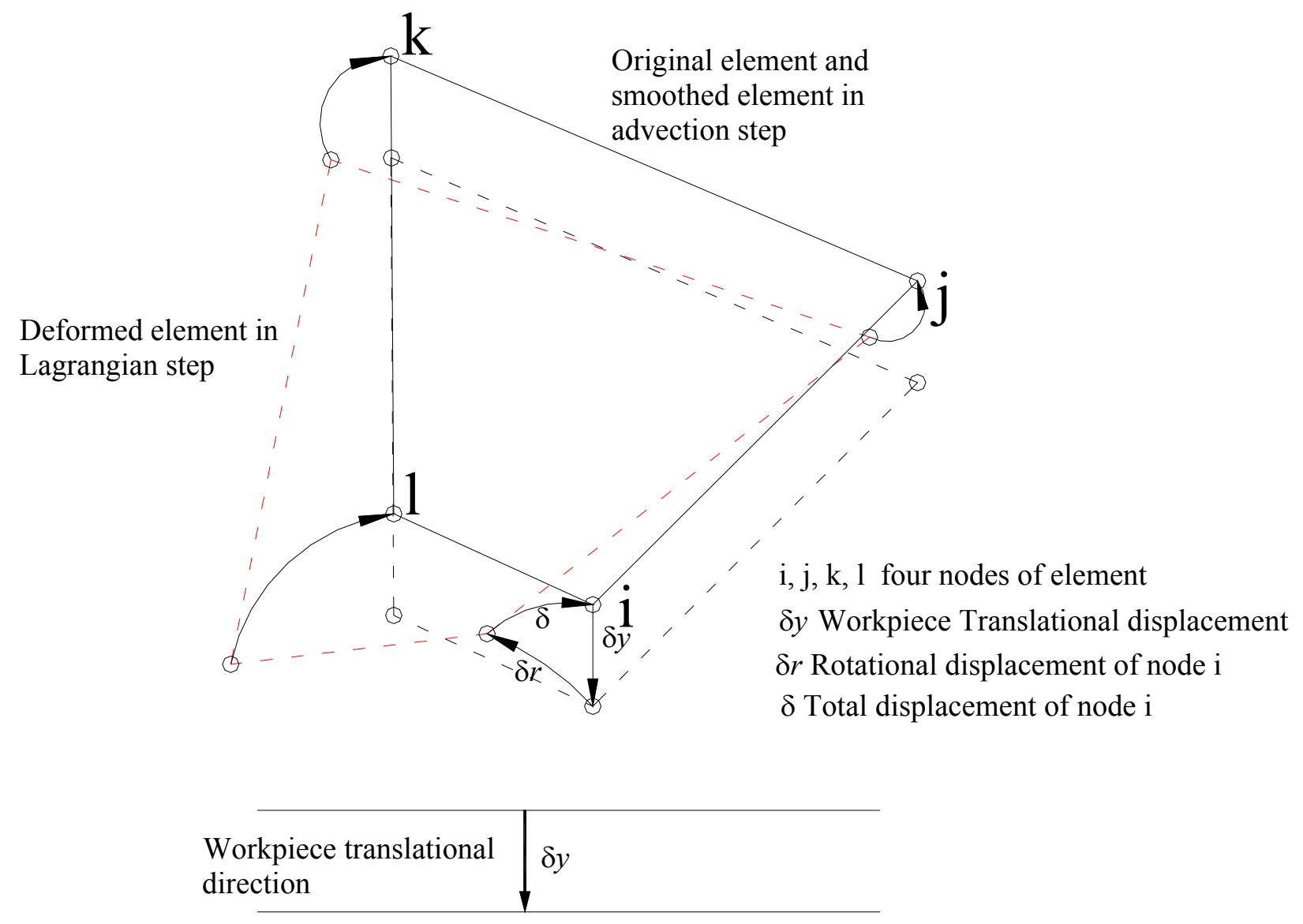

Figure 5-3 Mesh Motion of the Workpieces in the Moving Mesh Method 


\subsection{Applications of Moving Mesh to the ALE Modeling of FSW}

Four cases documenting the material flow in the friction stir butt weld of aluminum alloys are presented using the moving mesh method. As the first case, the FSW process modeled in the previous chapter with a modification of the loading conditions is simulated. This case is to illustrate the performance of the moving mesh method. Then the method is respectively applied to the FSW process characterized by the power law isotropic plastic and the elastic-perfectly plastic with kinematic hardening material models to show the effects of different material models on the material flow patterns. The third case, the FSW process for joining dissimilar aluminum alloys, is simulated using the same method to show the capability of the finite element model for the FSW of the dissimilar aluminum alloys. The last case, friction effects on the material flow pattern in the friction stir weld of the same aluminum alloy, is studied using the moving mesh method again.

\subsubsection{Case One - The Material Flow in the Same Aluminum Alloy Weld}

\subsubsection{Problem Definition}

The FSW process is simulated by a modification of the modeling performed in the previous chapter using the moving mesh method. The same geometries and materials are taken, and even the same translational and rotational velocities are prescribed. But in this problem, the welding tool rotates at a fixed position and the workpieces move with a translational velocity relative to the welding tool along the joint line.

The problem is defined in Fig.5-4. The pin and shoulder rotate with a rotational velocity in a fixed position and the boundaries of the workpieces is given a velocity equal 
to the translational velocity. It is noted that the welding tool still moves from down to up along the joint line relative to the workpiece material and also rotates in a counterclockwise direction. The advancing and retreating sides are indicated in the Fig.54 and their definitions are same as the previous chapter. The geometries, all reference values, and the chosen material properties are given in Tables 5-1 and 5-2, respectively. Again, the workpiece material is treated as an elastic-perfectly plastic with kinematic hardening model, and the pin and shoulder are assumed rigid.

The contact definitions are the same as the previous modeling. The boundary conditions are modified as that the degrees of freedom in the $\mathrm{x}$ (transverse) and $\mathrm{z}$ (depthwise) directions instead of the three directions are restricted along the circular edge of the workpieces. Again, the bottom of the workpiece is fixed along the $\mathrm{z}$ direction. 


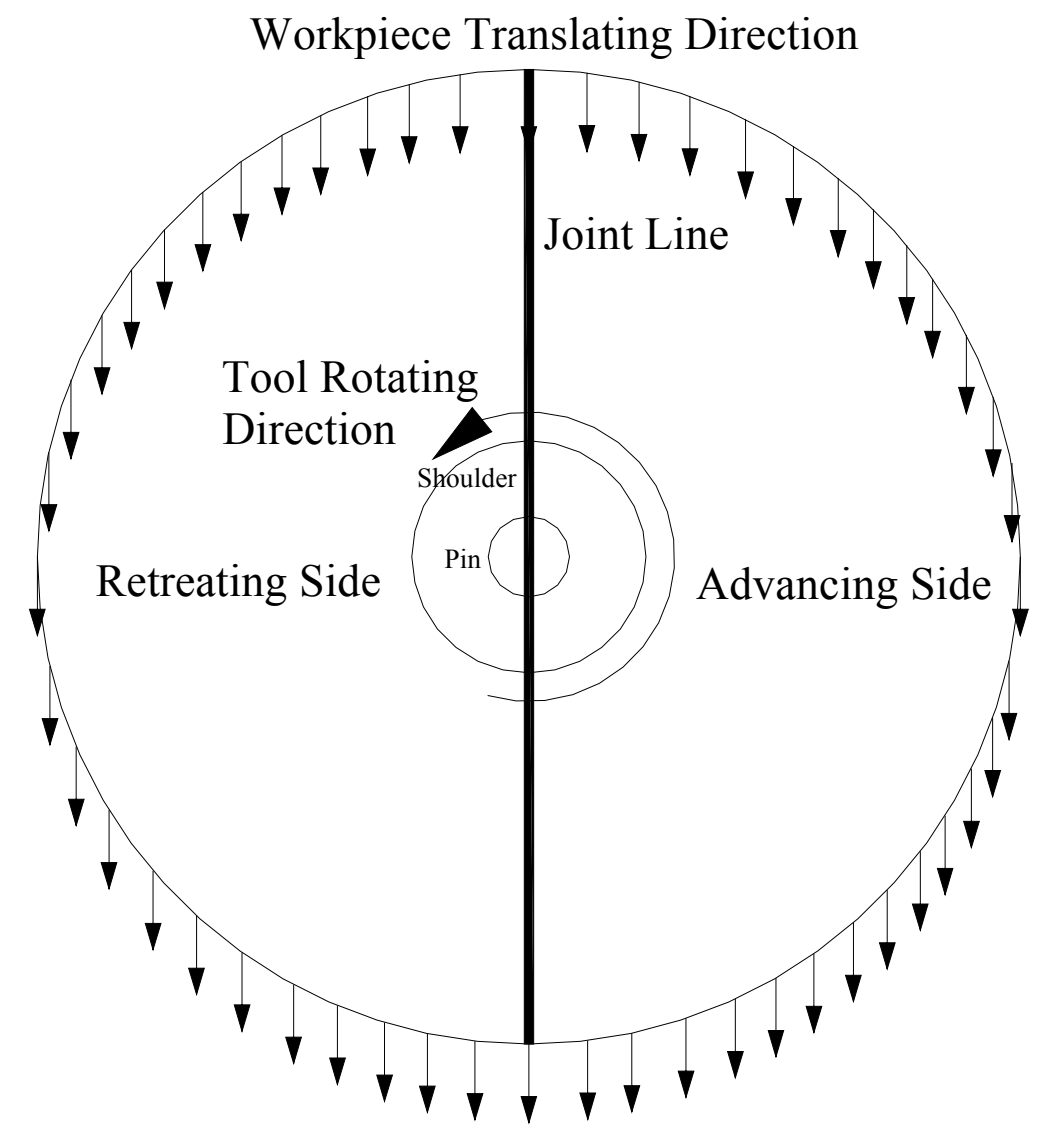

Figure 5-4 Problem Definition

Table 5-1 Geometries and Reference Values

\begin{tabular}{c|c|c|c}
\hline & Workpieces & Pin & Shoulder \\
\hline \hline Diameter (in) & 3.0 & 0.2552 & 0.7656 \\
\hline Thickness (in) & 0.125 & 0.12 & 0.05 \\
\hline Translational Velocity & \multicolumn{3}{|c}{$0.2 \mathrm{in} / \mathrm{sec}$} \\
\hline Rotational Velocity & \multicolumn{3}{|c}{$94.2 \mathrm{rad} / \mathrm{sec}(900 \mathrm{rpm})$} \\
\hline Frictional Coefficient & \multicolumn{3}{|c}{0.47} \\
\hline Forging & \multicolumn{3}{|c}{$0.005 \mathrm{in}$} \\
"Force"(Displacement) &
\end{tabular}


Table 5-2 Mechanical Properties of Material

\begin{tabular}{c|c|c|c|c}
\hline Material & $\begin{array}{c}\text { Mass Density } \\
\left(\left(\mathrm{b}^{*} \mathrm{~s}^{\wedge} 2\right) / \mathrm{in}^{\wedge} 4\right)\end{array}$ & $\begin{array}{c}\text { Young's } \\
\text { Modulus } E \\
(\mathrm{psi})\end{array}$ & $\begin{array}{c}\text { Poisson } \\
\text { Ratio }\end{array}$ & $\begin{array}{c}\text { Yield Stress } \\
\sigma_{y}(\mathrm{psi})\end{array}$ \\
\hline $\begin{array}{c}\text { Workpieces } \\
(\text { Al 6061-T6) }\end{array}$ & 0.00025 & $1.0 \mathrm{e} 7$ & 0.33 & 39900 \\
\hline $\begin{array}{c}\text { Pin and shoulder } \\
\text { (Steel) }\end{array}$ & 0.00073 & $3.0 \mathrm{e} 7$ & 0.28 & ---- \\
\hline
\end{tabular}

\subsubsection{Simulation Results}

\section{Volume Fraction Functions}

In Fig.5-5, the volume fraction contours for the workpieces at $t=0.05 \mathrm{~s}, 0.1 \mathrm{~s}$, $0.15 s, 0.2 s, 0.25 s, 0.3 s, 0.35 s, 0.4 s, 0.45 s, 0.5 s$ are presented. In the figure, the red and blue shades represent the two workpieces. The advancing side is on the right and the retreating side is on the left. The pin and shoulder are displayed in the green and yellow feature lines, respectively. The material moving direction and the tool rotating direction are indicated in the first image.

Based on the volume fraction contours, first, it is shown that the hole in the middle of the workpieces remains right at the location of the pin as the process proceeds due to the fact that the method of ALE formulations with moving mesh used in the model. Thus, the issue discussed in the beginning of this chapter is resolved in this model.

Second, the contours show how the workpiece material moves around the pin. By the rotation of the welding tool, the material in the advancing side moves around the pin to the retreating side in the rotational direction of the tool and then moves toward the 
behind of the pin. After the material arrives at the behind of the pin, part of material is transported further along the workpiece moving direction and part of material moves back to the advancing side. The material in the retreating side is also transported around the pin to the advancing side in the rotational direction of the tool, then moves against the workpiece moving direction on the advancing side, and then goes back to the retreating side. The material around the pin follows such a motion procedure and finally a friction stir weld is formed and left behind the welding tool. It is apparent that the material flow is not symmetric about the joint line. The flow patterns on the advancing side and retreating sides are different.

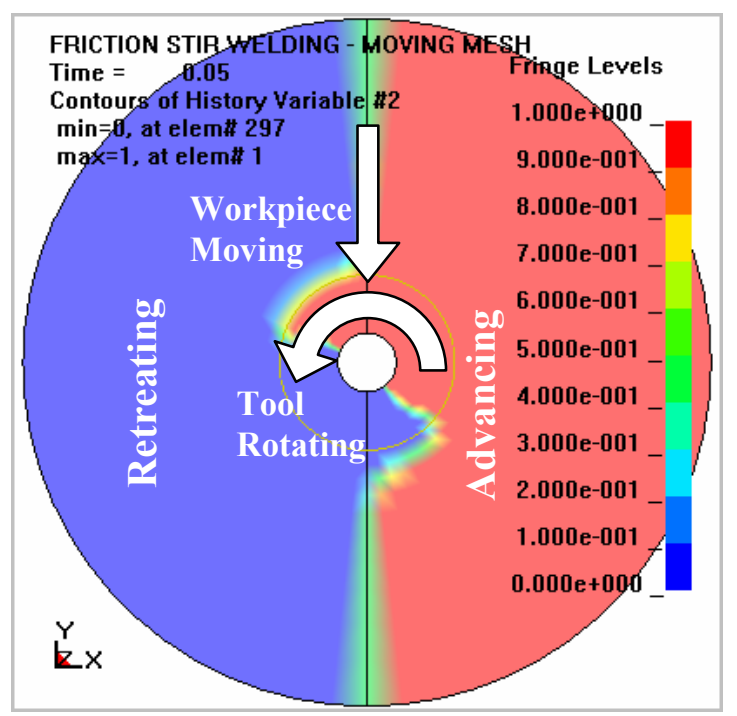

$t=0.05 s$

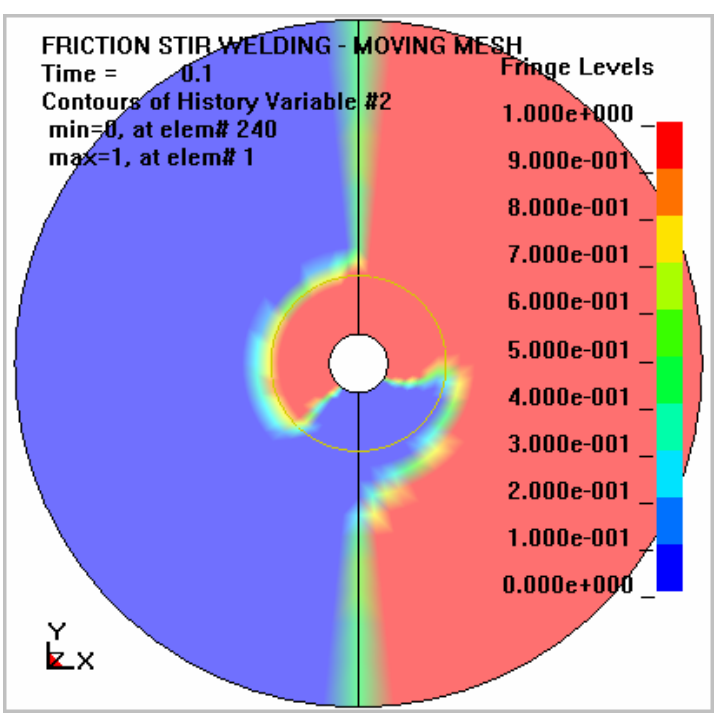

$t=0.1 \mathrm{~s}$ 


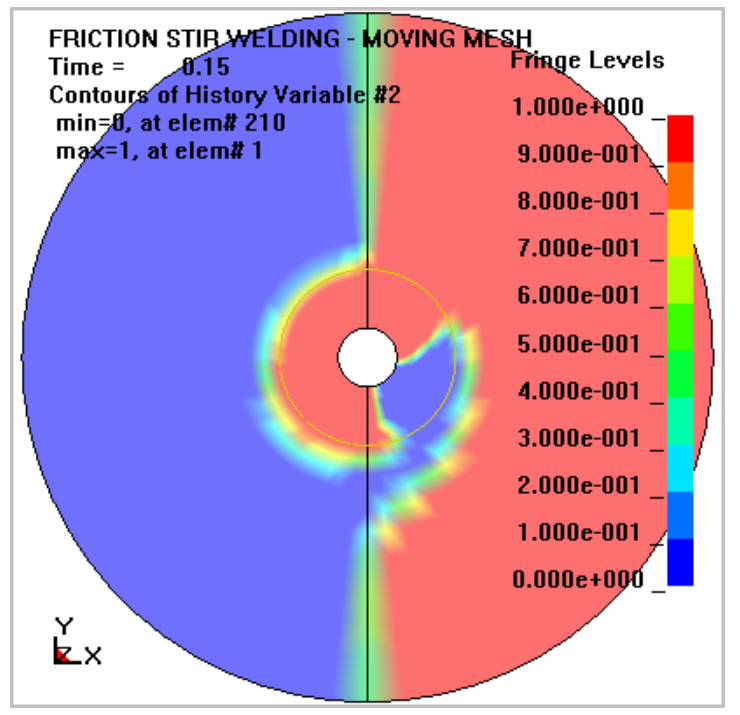

$$
t=0.15 \mathrm{~s}
$$

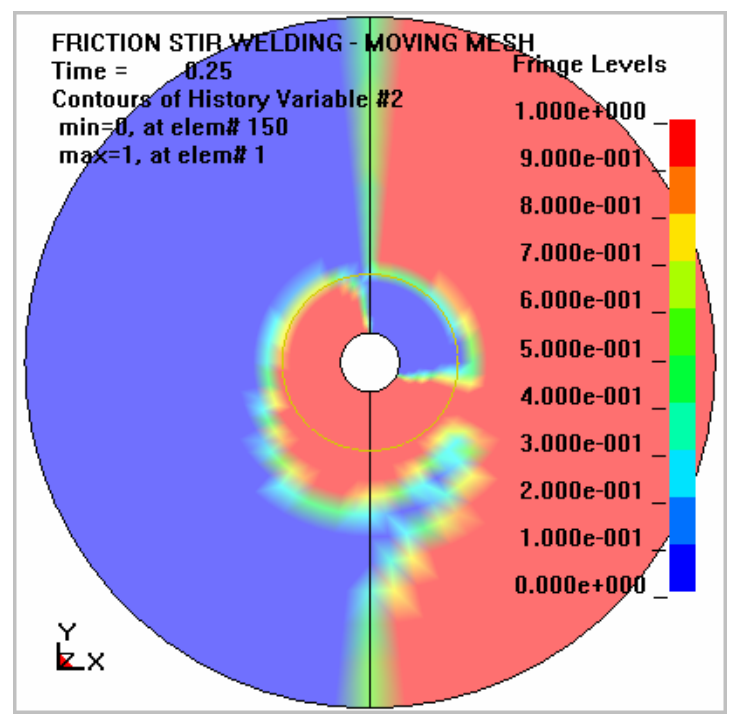

$t=0.25 s$

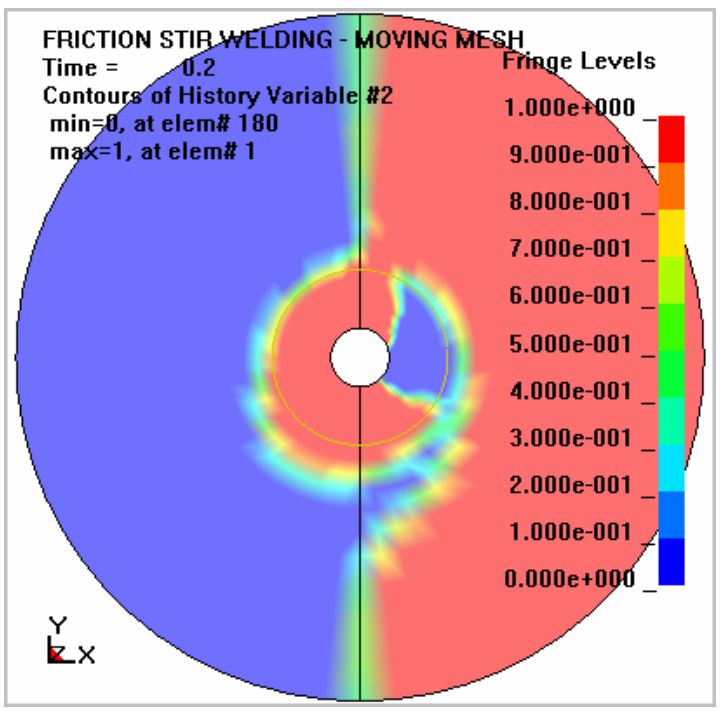

$$
t=0.2 \mathrm{~s}
$$

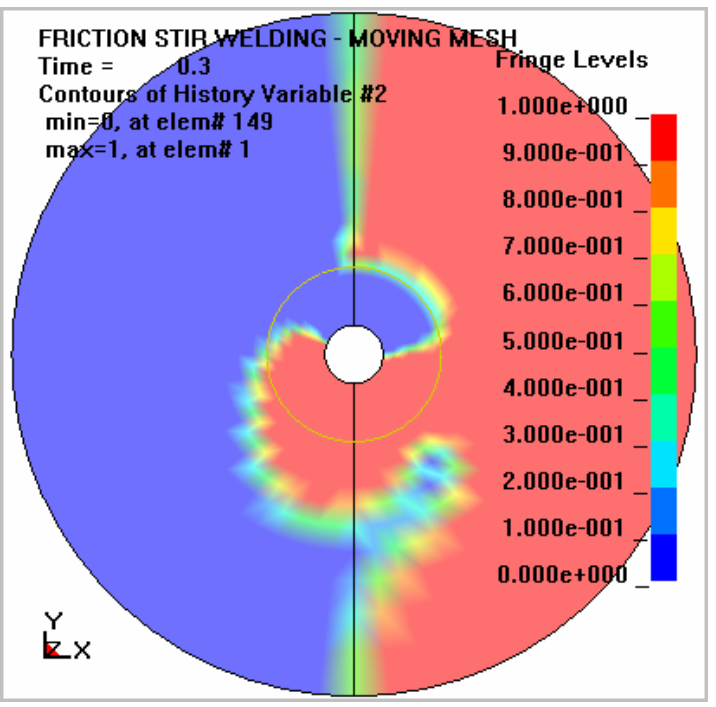

$$
t=0.3 s
$$




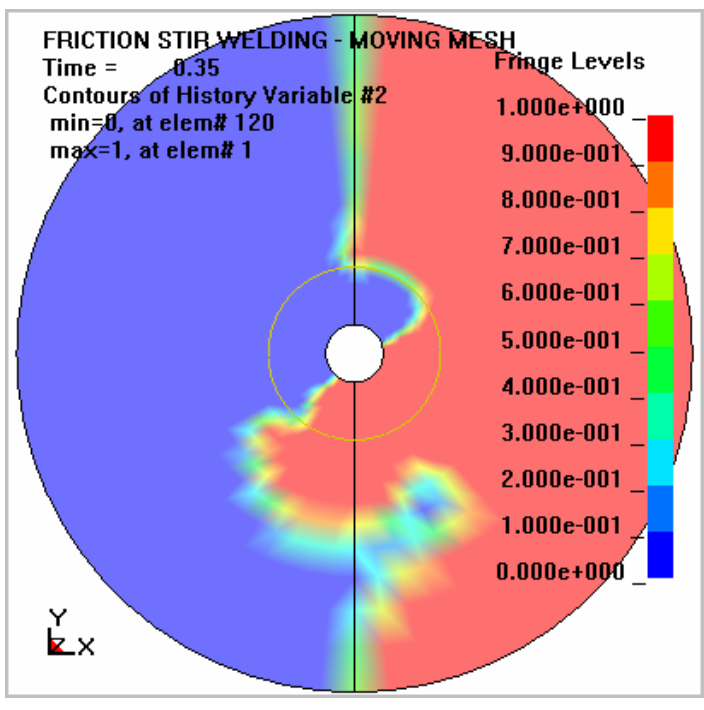

$$
t=0.35 \mathrm{~s}
$$

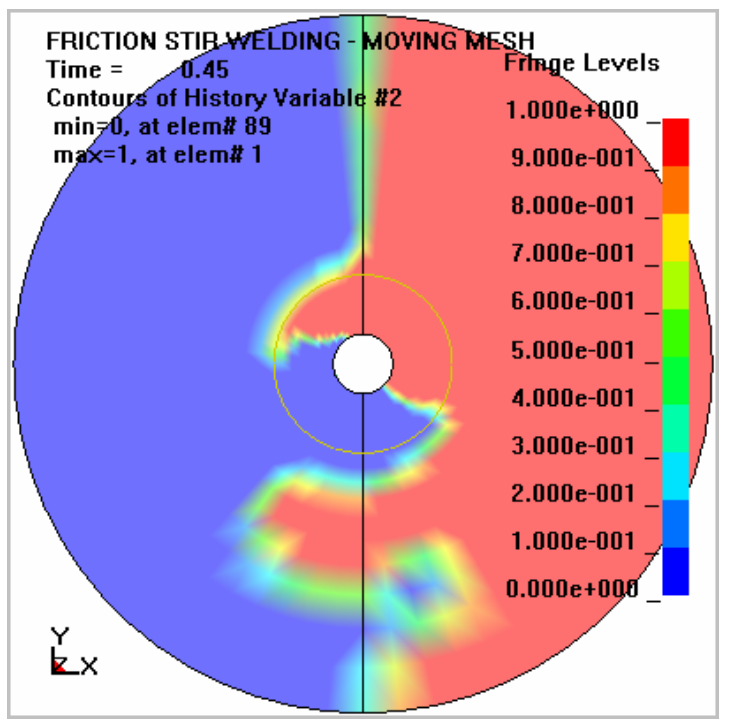

$$
t=0.45 s
$$

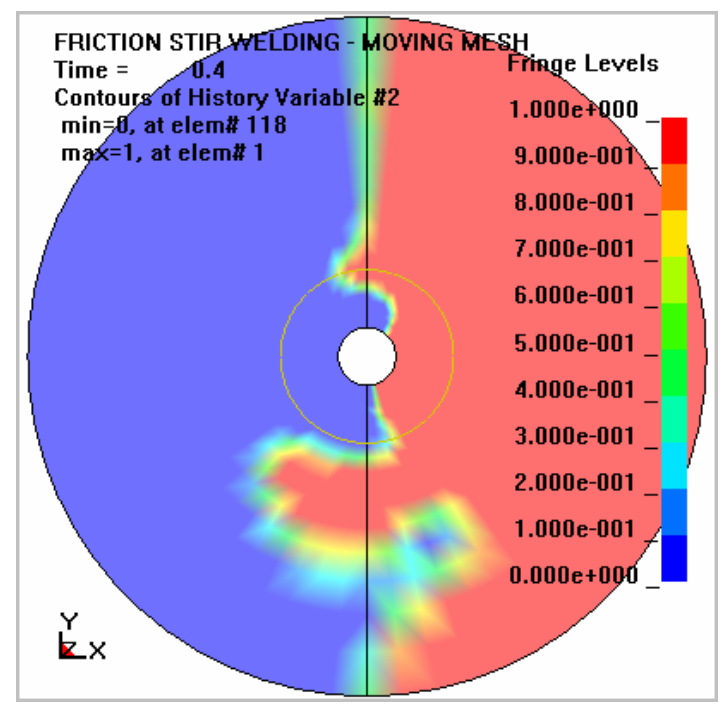

$$
t=0.4 s
$$

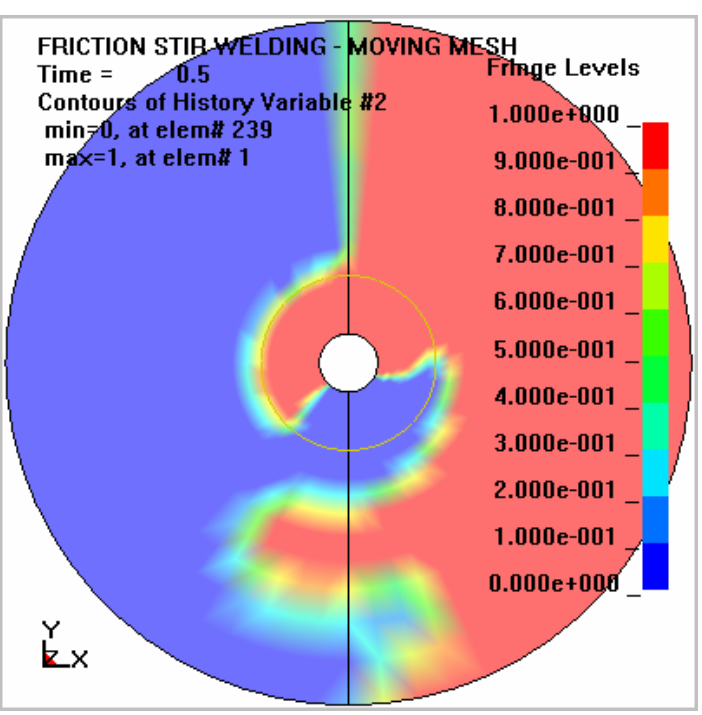

$$
t=0.5 s
$$

Figure 5-5 Volume Fraction Contours for the Workpieces at Different Time Instances 


\section{Tracer Tracking Method}

An alternative approach to visualize the motion of material during the FSW process is to define tracers to track the history of the material points starting from any initial location in the workpieces as they flow around the tool. The history includes positions, velocities and stresses of the material points that the tracers follow. In this model, eighty-eight tracers are defined, which are initially located at the 45 deg., 135 deg., 225 deg., 315 deg., 0 deg., 90 deg., 180 deg. and 270 deg. radial lines, at the top surface of the workpieces $(\mathrm{Z}$ coordinates $=0)$, within the shoulder diameter where the material flow pattern is of interest. But only the motions of the tracers at the 45 deg., 135 deg., 225 deg. and 315 deg. lines are discussed in detail in this chapter. The motions of other tracers on the weld line and the line perpendicular to the weld line will be discussed in Chapter 6.

The initial positions for those tracers at the 45 deg., 135 deg., 225 deg. and 315 deg. radial lines are indicated in Fig.5-6. In the figure, the green shades represent the pin and the yellow feature line is the shoulder and the defined tracers are within the shoulder diameter. The initial coordinates for those tracers are listed in Table 5-3. The planar and vertical (depth-wise) motions of tracer T1-T11 (on the $45 \mathrm{deg}$. line), T12-T22 (on the 315 deg. line), T23-T33 (on the 135 deg. line) and T34-T44 (on the 225 deg. line) will be discussed respectively. 


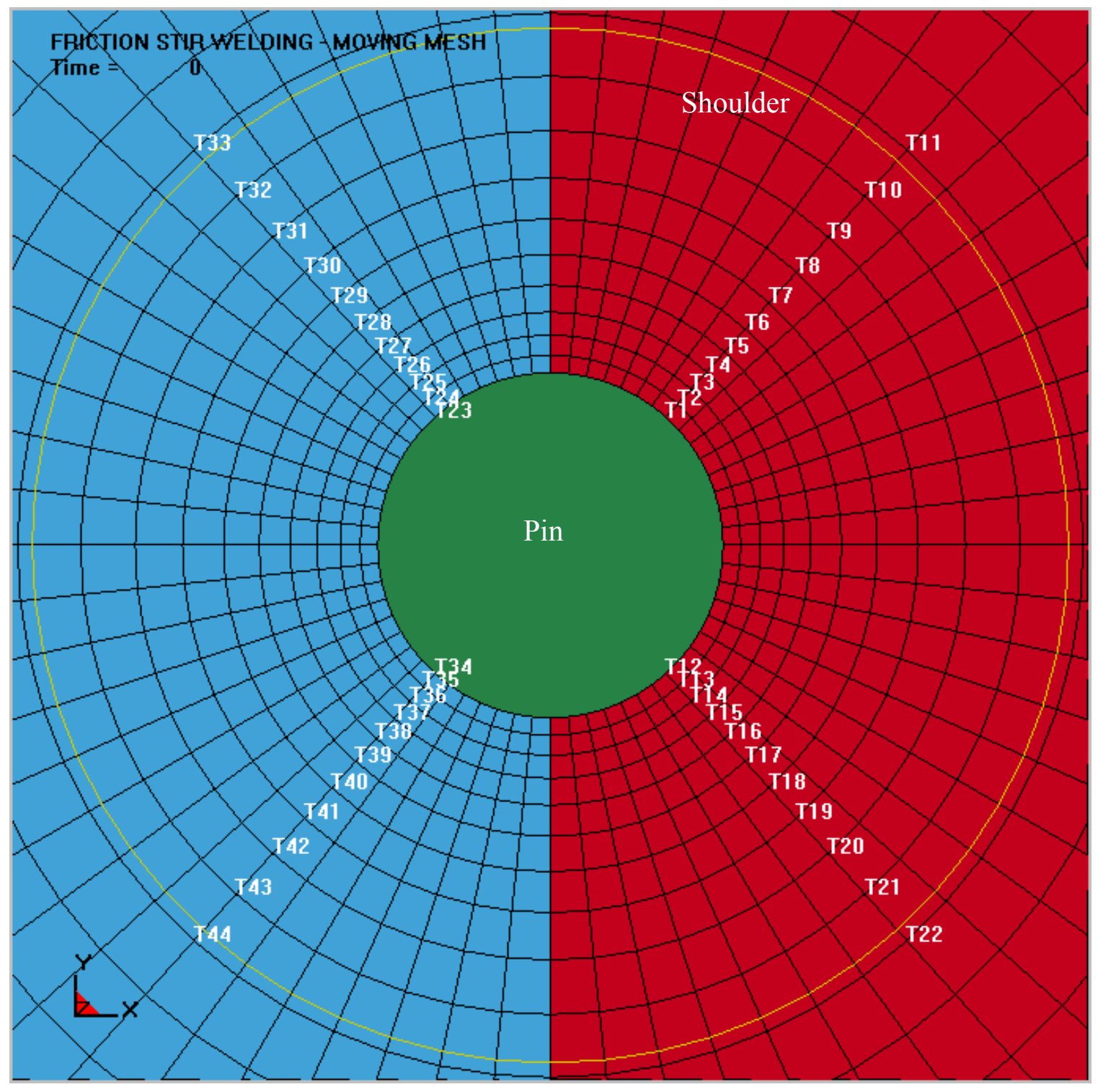

Txx is a Tracer

Figure 5-6 Forty-four Tracers at the Top Surface of the Workpieces within the Shoulder Diameter are Defined in the Model 
Table 5-3 Initial Coordinates of the Tracers

\begin{tabular}{|c|c|c|c|}
\hline Tracers & $\mathbf{X}$ & $\mathbf{Y}$ & $\mathbf{Z}$ \\
\hline T 1 & 0.08538 & 0.09483 & 0.0 \\
\hline T 2 & 0.09401 & 0.1044 & 0.0 \\
\hline T 3 & 0.1040 & 0.1155 & 0.0 \\
\hline T 4 & 0.1154 & 0.1282 & 0.0 \\
\hline T 5 & 0.1287 & 0.1429 & 0.0 \\
\hline T 6 & 0.1439 & 0.1599 & 0.0 \\
\hline T 7 & 0.1615 & 0.1794 & 0.0 \\
\hline T 8 & 0.1818 & 0.2020 & 0.0 \\
\hline T 9 & 0.2053 & 0.2280 & 0.0 \\
\hline T 10 & 0.2323 & 0.2579 & 0.0 \\
\hline T 11 & 0.2654 & 0.2925 & 0.0 \\
\hline T 12 & 0.08538 & -0.09483 & 0.0 \\
\hline T 13 & 0.09401 & -0.1044 & 0.0 \\
\hline T 14 & 0.1040 & -0.1155 & 0.0 \\
\hline T 15 & 0.1154 & -0.1282 & 0.0 \\
\hline T 16 & 0.1287 & -0.1429 & 0.0 \\
\hline T 17 & 0.1439 & -0.1599 & 0.0 \\
\hline T 18 & 0.1615 & -0.1794 & 0.0 \\
\hline T 19 & 0.1818 & -0.2020 & 0.0 \\
\hline T 20 & 0.2053 & -0.2280 & 0.0 \\
\hline T 21 & 0.2323 & -0.2579 & 0.0 \\
\hline T 22 & 0.2654 & -0.2925 & 0.0 \\
\hline T 23 & -0.08538 & 0.09483 & 0.0 \\
\hline T 24 & -0.09401 & 0.1044 & 0.0 \\
\hline T 25 & -0.1040 & 0.1155 & 0.0 \\
\hline T 26 & -0.1154 & 0.1282 & 0.0 \\
\hline T 27 & -0.1287 & 0.1429 & 0.0 \\
\hline T 28 & -0.1439 & 0.1599 & 0.0 \\
\hline T 29 & -0.1615 & 0.1794 & 0.0 \\
\hline T 30 & -0.1818 & 0.2020 & 0.0 \\
\hline T 31 & -0.2053 & 0.2280 & 0.0 \\
\hline T 32 & -0.2323 & 0.2579 & 0.0 \\
\hline T 33 & -0.2654 & 0.2925 & 0.0 \\
\hline T 34 & -0.08538 & -0.09483 & 0.0 \\
\hline T 35 & -0.09401 & -0.1044 & 0.0 \\
\hline T 36 & -0.1040 & -0.1155 & 0.0 \\
\hline T 37 & -0.1154 & -0.1282 & 0.0 \\
\hline T 38 & -0.1287 & -0.1429 & 0.0 \\
\hline T 39 & -0.1439 & -0.1599 & 0.0 \\
\hline T 40 & -0.1615 & -0.1794 & 0.0 \\
\hline T 41 & -0.1818 & -0.2020 & 0.0 \\
\hline T 42 & -0.2053 & -0.2280 & 0.0 \\
\hline T 43 & -0.2323 & -0.2579 & 0.0 \\
\hline T 44 & -0.2654 & -0.2925 & 0.0 \\
\hline
\end{tabular}


The initial positions of tracer T1-T11 in the model are shown in Fig.5-7(a) and their planar and vertical (depth-wise) positions at $t=0.5 \mathrm{~s}$ are shown in Fig.5-7(b) and (c), respectively. In the figure, the blue and red shades are the two workpieces, the green shades are the pin, and the yellow shades and yellow feature line both represent the shoulder. The material moving and tool rotating directions are shown in Fig.5-7(a).

In Fig.5-7(b), it is shown that the material particles initially located at the advancing side move around the pin in the rotational direction of the tool when they make contact with the welding tool. After the FSW process proceeds $0.5 \mathrm{~s}$, material particle T4, T6, $\mathrm{T} 7$ and $\mathrm{T} 8$ deposit on the retreating side and $\mathrm{T} 1, \mathrm{~T} 2, \mathrm{~T} 3, \mathrm{~T} 5, \mathrm{~T} 9, \mathrm{~T} 10, \mathrm{~T} 11$ remain on the advancing side but their positions are different from the original. The figure also shows, during the FSW process, some material particles T1, T2, T4, T6, T9, T10 are carried more than once around the rotating pin.

In Fig.5-7(c), it is shown that most material particles except T2, T4 and T11 move downward toward the bottom of the weld from the original position.

To help understand the planar and vertical (depth-wise) motions of those material particles shown in Fig.5-7(b) and (c), X\&Y, Z coordinates for tracer T1-T11 over time are plotted in Figs.5-8 and 5-9, respectively. In this and following figures, the positive X values mean that the material particles are on the advancing side, while the negative $X$ values mean that the material particles are on the retreating side. Positive $\mathrm{Y}$ values mean that the material particles are on the upper portion (in the XY plane) while the negative $\mathrm{Y}$ values mean that the material particles are on the lower portion. The positive $Z$ values mean that the material particles move upward from the top surface of the workpieces, 
while the negative $\mathrm{Z}$ values mean that the material particles move downward from the top surface of the workpieces.

Fig.5-8(a)-(k) show the $\mathrm{X}$ and $\mathrm{Y}$ coordinates of T1-T11 over time, respectively. For the purpose of clear observation, only the values in a segment of $0 s-0.1 s$ are plotted for each tracer particle. Actually, the process is already stable after $0.1 \mathrm{~s}$. In Fig.(a), (b), (d), (f), (i), (j), it is shown that, during FSW, material particle T1, T2, T4, T6, T9, T10 rotate with the welding tool after they get on the pin or shoulder, as can be seen in Fig.57(b). Other particles including T3, T5, T7, T8 and T11 stop moving after they arrive at some location.

Fig.5-9 shows the Z coordinates over time. Particle A1-K11 represent tracer T1T11, respectively. All the curves show that the $Z$ coordinate starts with zero, then decreases to some value, then goes up to a constant value, this constant value is negative in the most curves but is positive for curve T2, T4 and T11, as shown in Fig.5-7(c). This phenomenon can be explained as follows: at the beginning of welding, the material is pushed down by the shoulder and pin. Since FSW is a constant- volume process and the shoulder, the pin and undeformed base material restrict the flow path, when the material is transported closer to the bottom of the weld, the material transport is restricted by the backing plate, it is then forced upward. But this up motion cannot last for a long time since the welding tool acts on the workpieces, and finally they do not go down and up and stay a fix position. In addition, it is noted that the up motion is strongest in a narrow region surrounding the pin and shoulder. That is the reason why T2, T4 and T11 go upward from the top surface of the workpieces. 


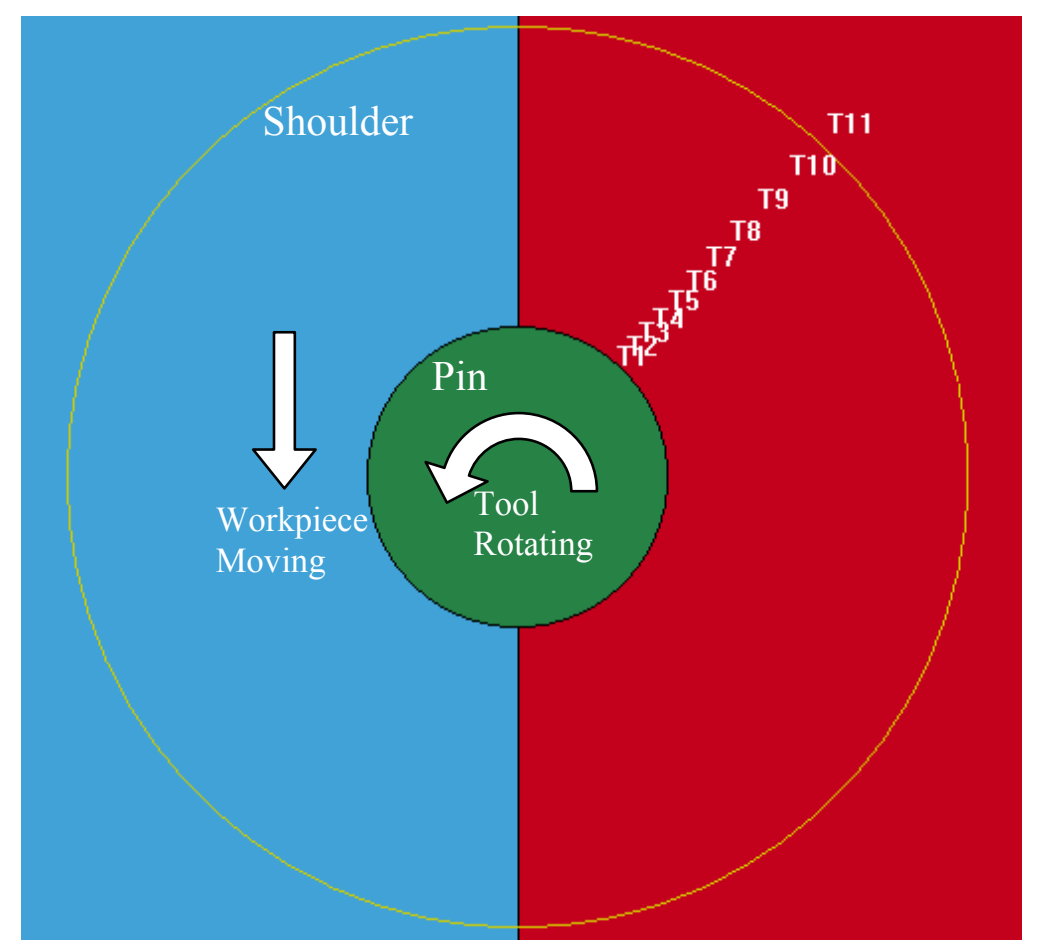

(a) Initial Positions

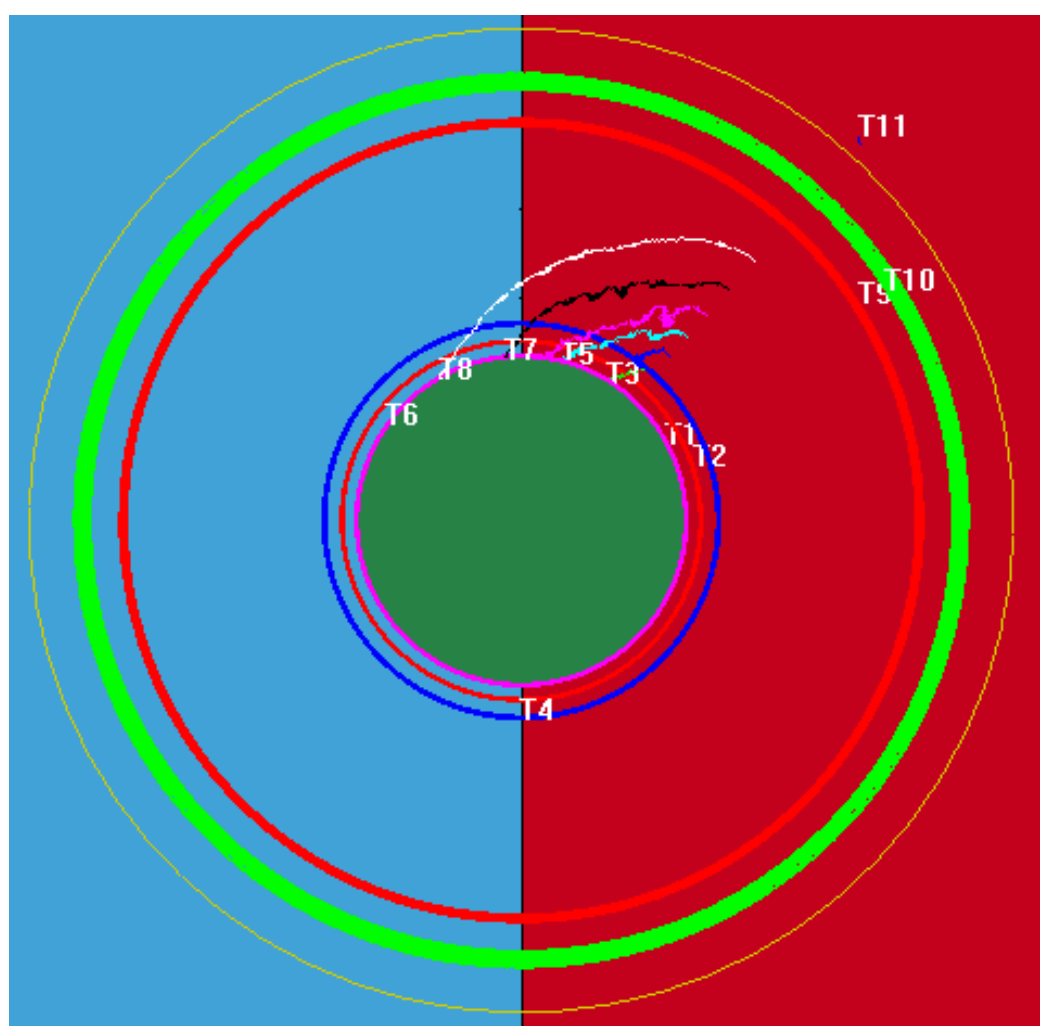

(b) Planar Positions at $t=0.5 \mathrm{~s}$ 


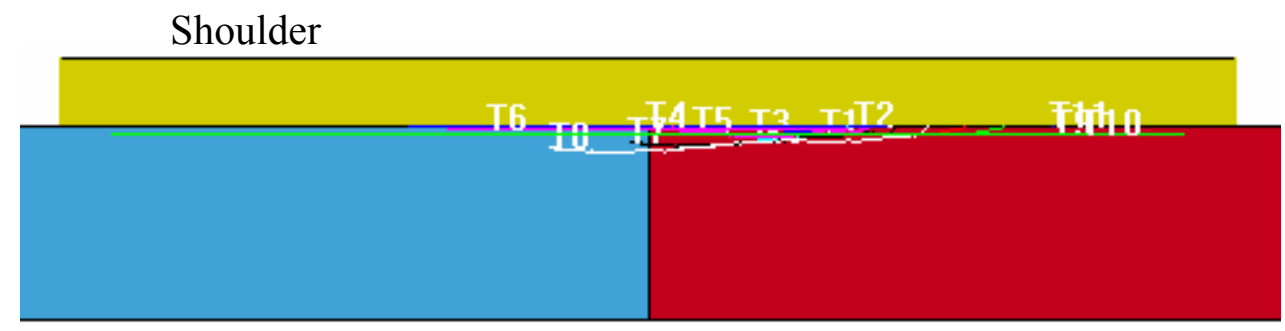

(c)Vertical (Depth-wise) Positions at $t=0.5 \mathrm{~s}$

Figure 5-7 Planar and Vertical (Depth-wise) Motions of Tracer T1-T11 in the Model

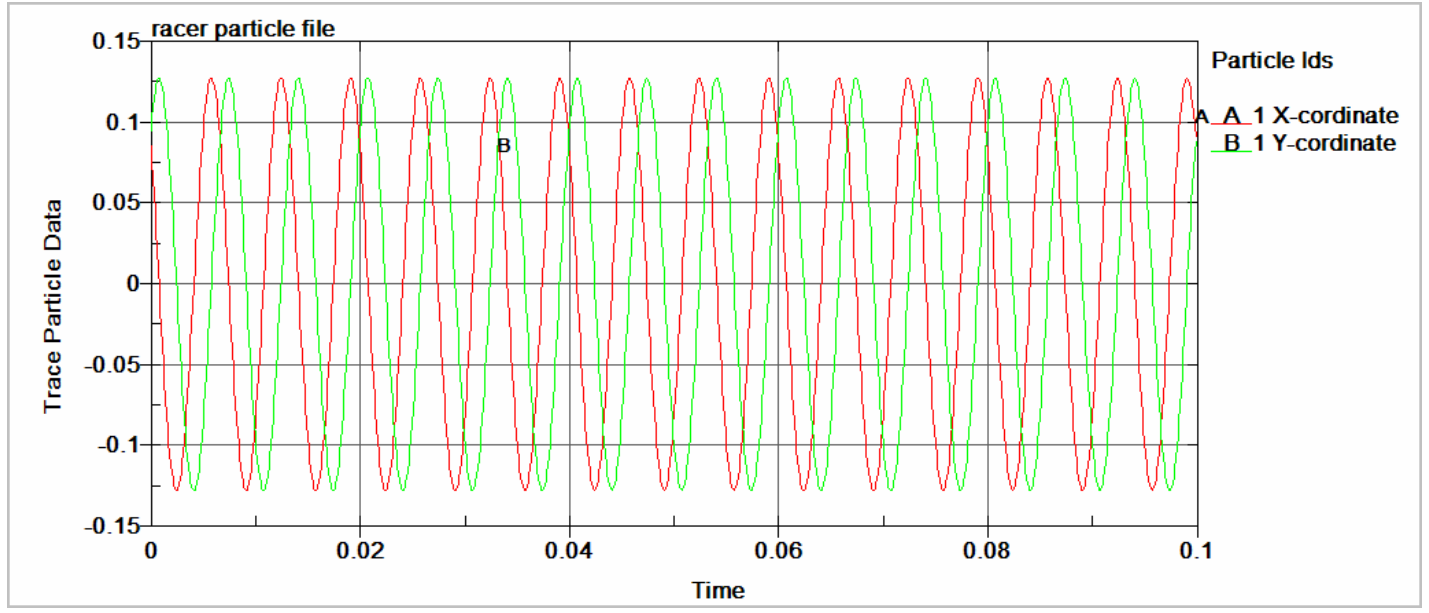

(a) T1

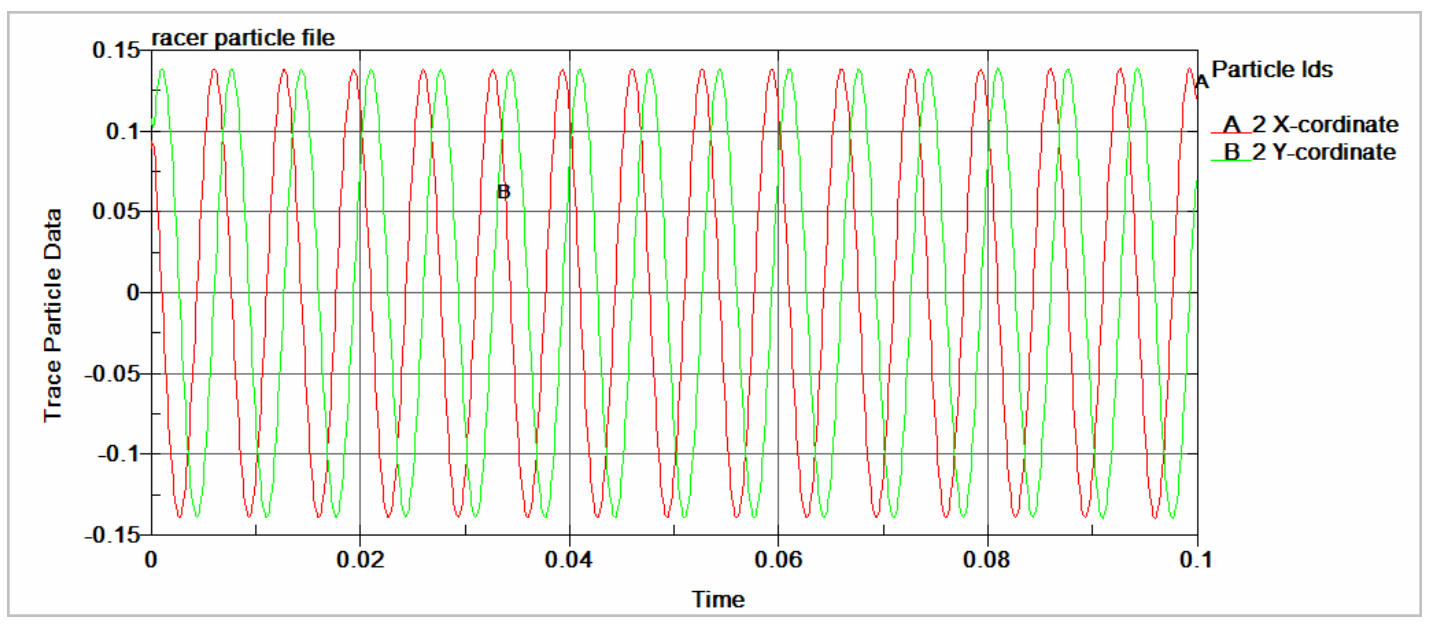

(b) $\mathrm{T} 2$ 


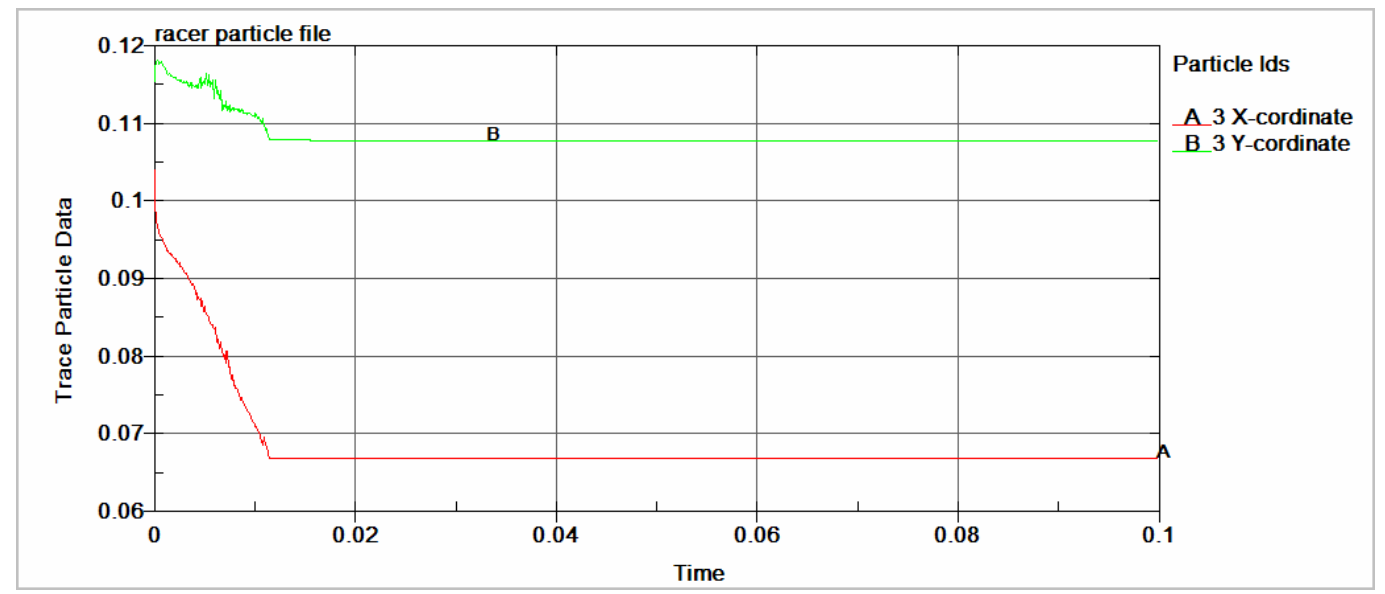

(c) $\mathrm{T} 3$

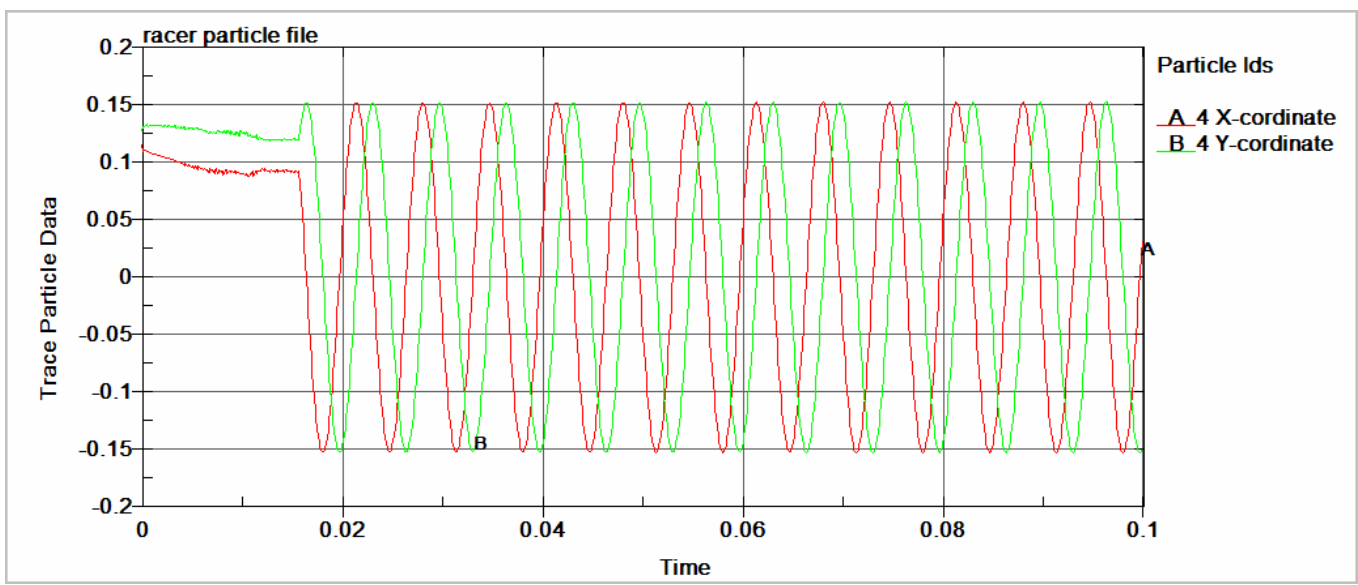

(d) $\mathrm{T} 4$

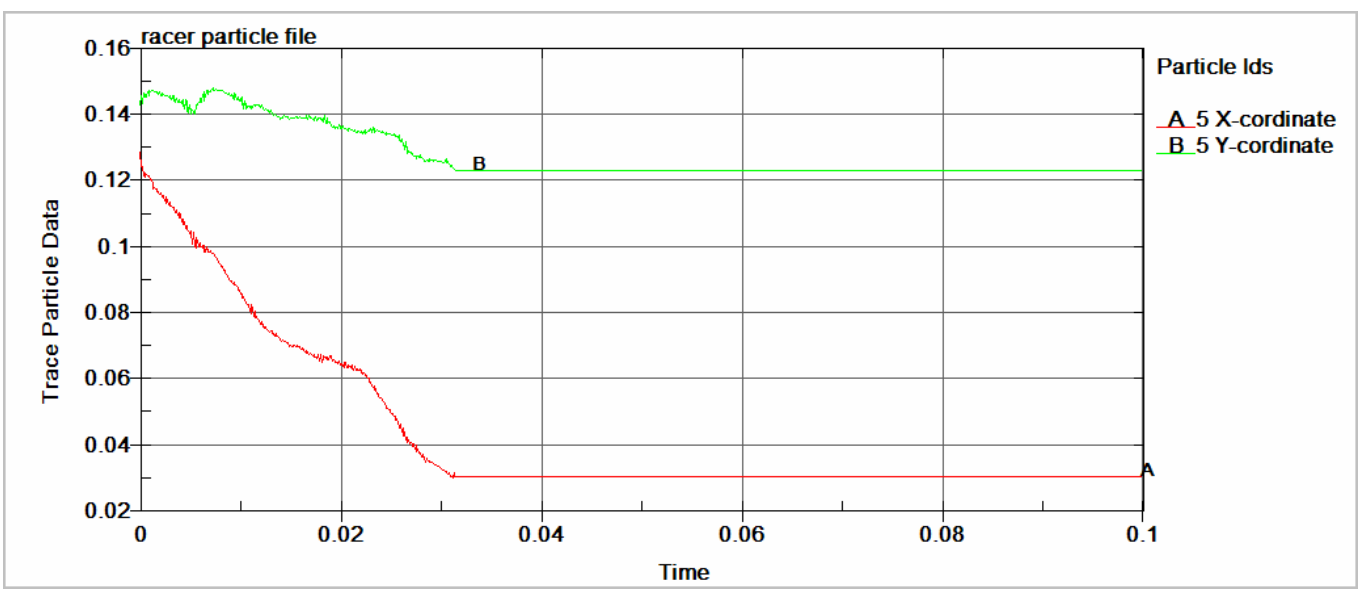

(e) $\mathrm{T} 5$ 


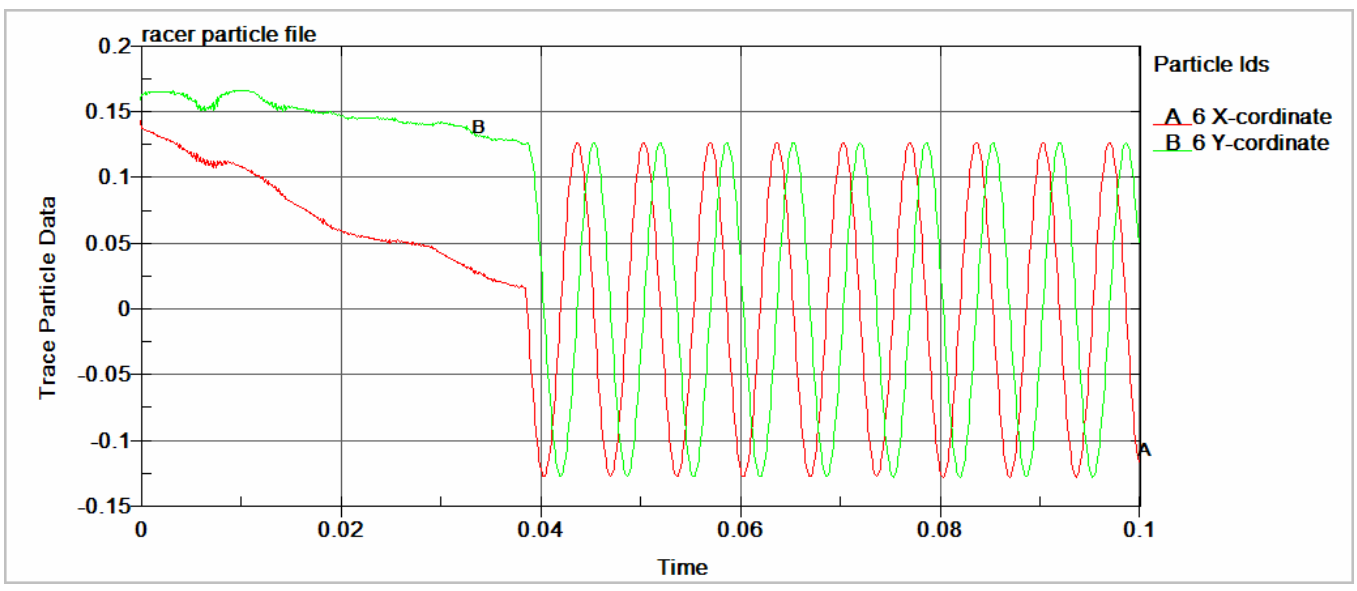

(f) $\mathrm{T} 6$

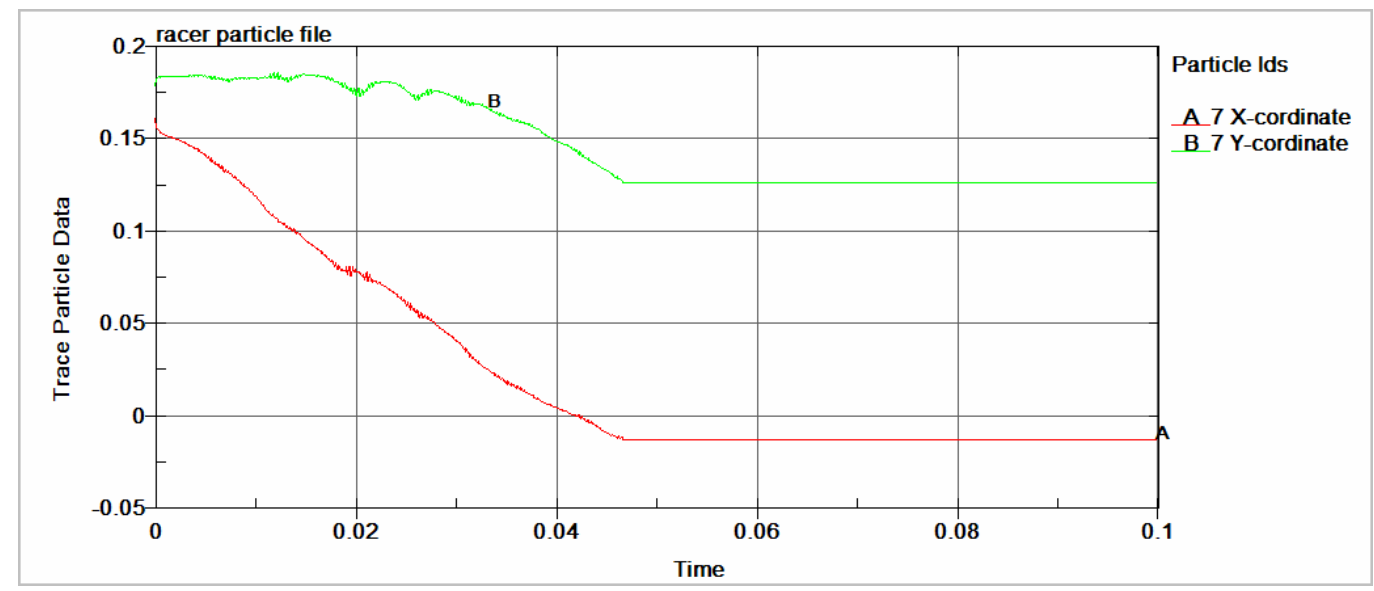

(g) $\mathrm{T} 7$

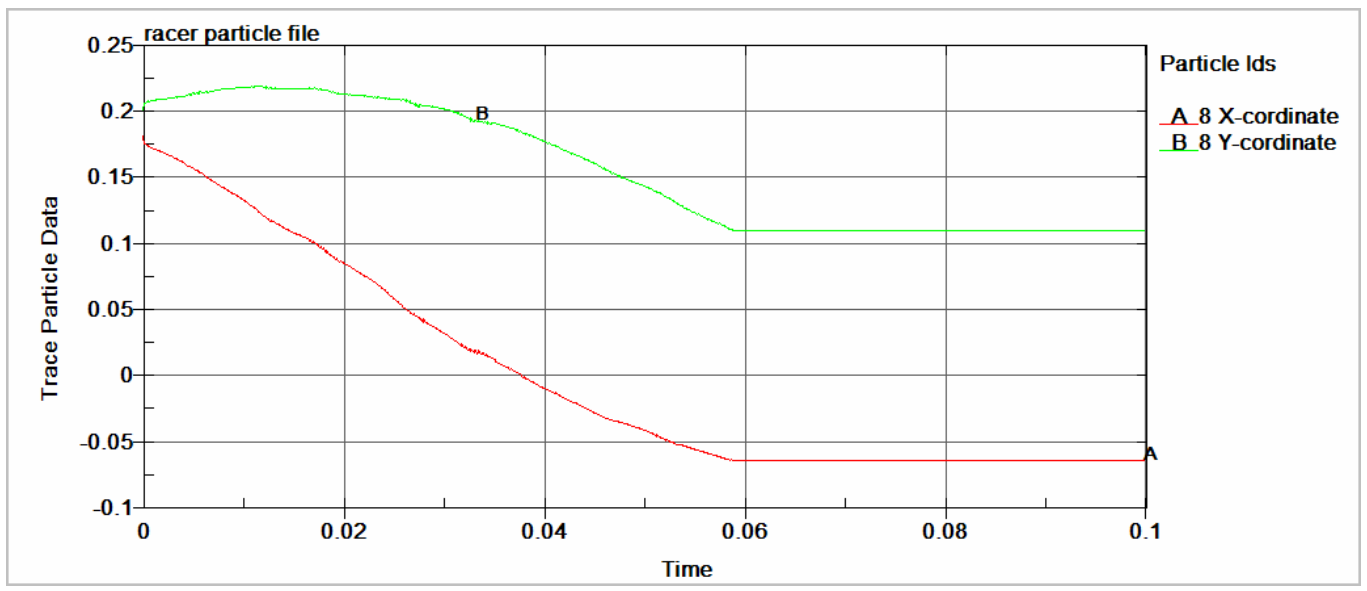

(h) $\mathrm{T} 8$ 


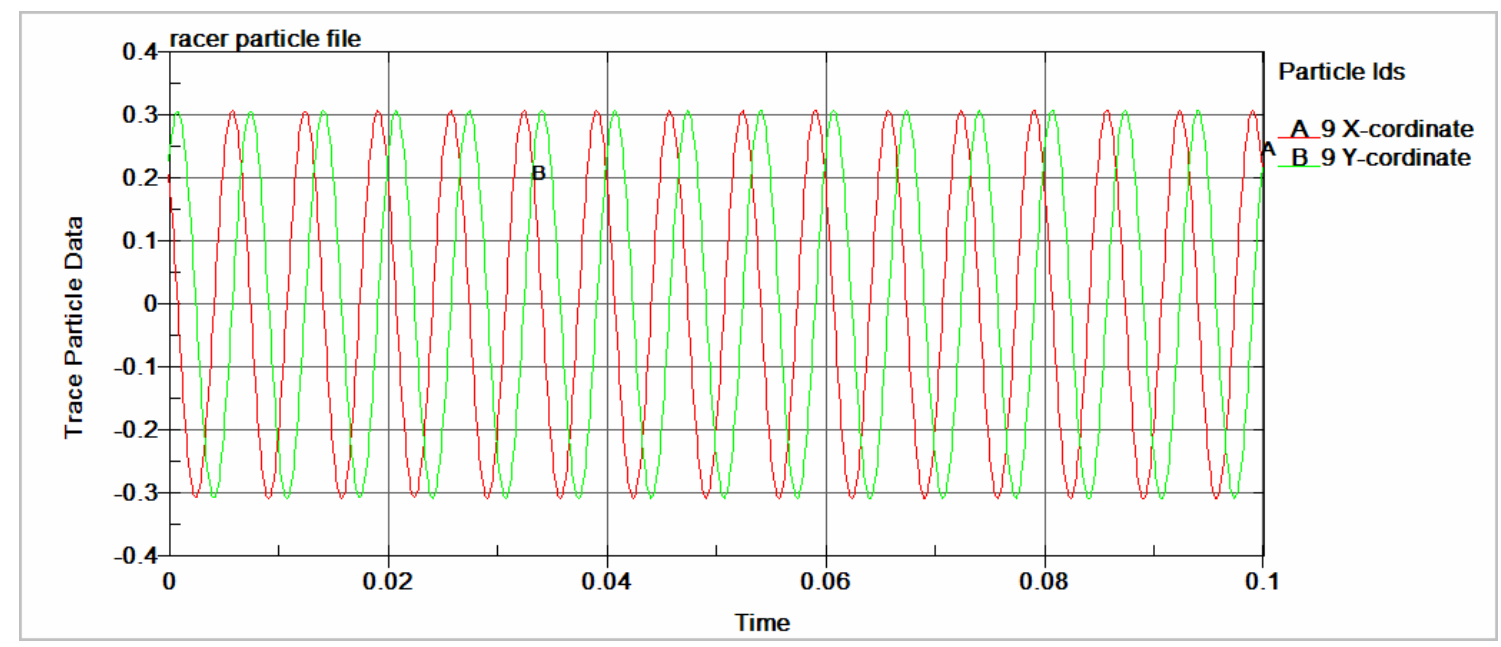

(i) $\mathrm{T} 9$

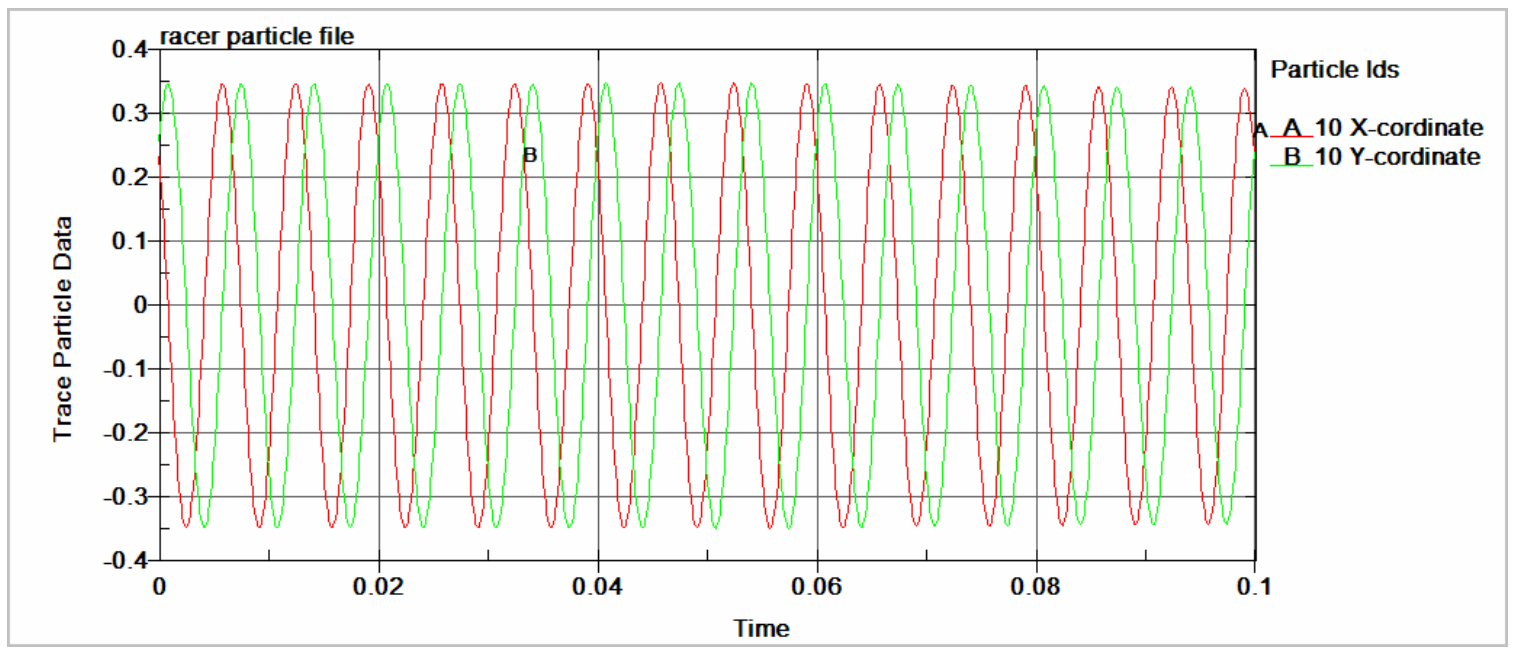

(j) $\mathrm{T} 10$ 


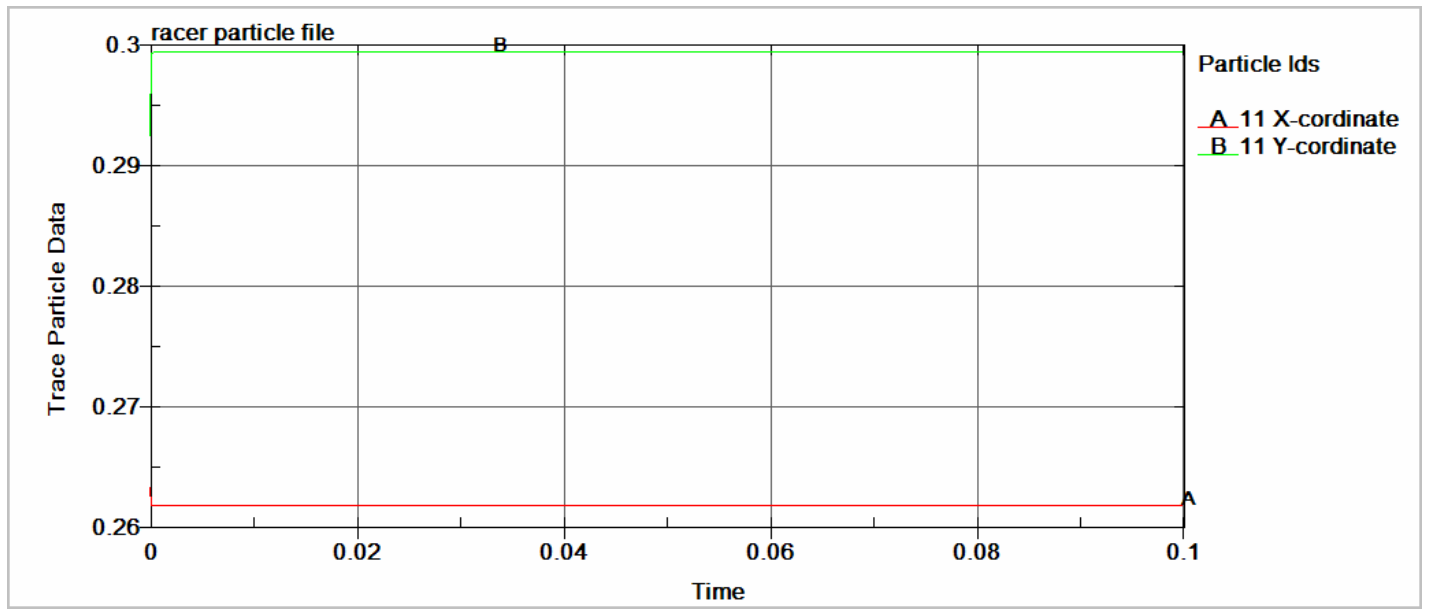

(k) T11

Figure 5-8 X and Y Coordinates vs. Time (a Segment of 0 s-0.1 s) for Tracer T1-T11

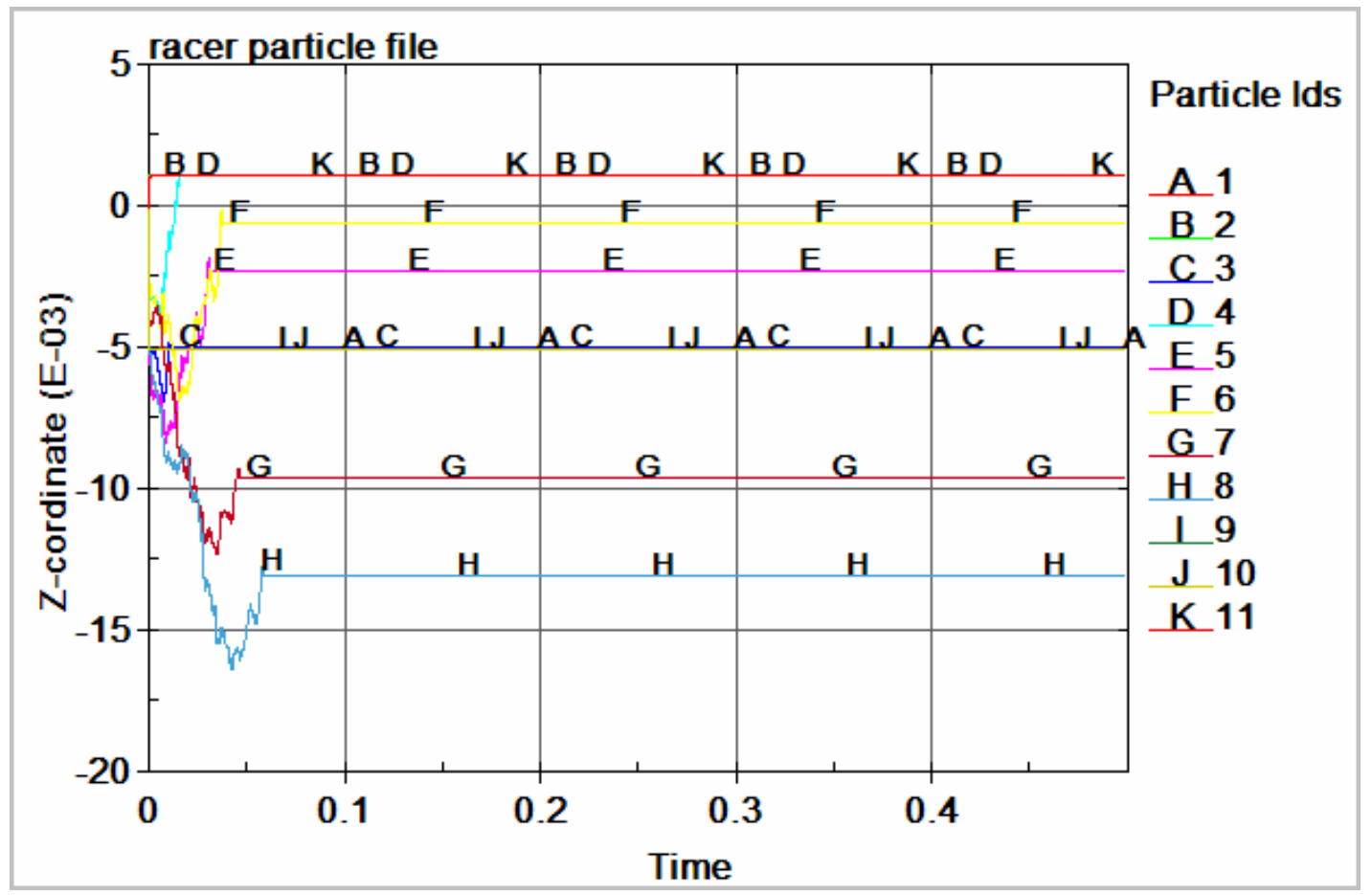

Figure 5-9 Z Coordinates vs. Time for Tracer T1-T11 
The same method is used to characterize the material flow patterns of tracer T12T22. The initial positions of tracer T12-T22 in the model are shown in Fig.5-10(a), and their planar and vertical (depth-wise) positions at $t=0.5 \mathrm{~s}$ are plotted in Fig.5-10(b) and (c), respectively. Z coordinates for those tracers over time are plotted in Fig.5-11. Particle A12-K22 represent tracer T12-T22, respectively.

In Fig.5-10(b), it is also shown that, at the beginning of FSW, the material particles originally located at the advancing side move around the pin in the rotational direction of the tool. After the FSW process precedes $0.5 \mathrm{~s}$, particle T13 and T14 move to the retreating side and other particles are still on the advancing side. During FSW, particle T15, T20, T22 stop moving when they arrive at some place and other particle keep rotating with the welding tool because they get on the pin or shoulder at some time.

In Fig.5-11, again, the curves show that the $\mathrm{Z}$ coordinate starts with zero and decreases to some value, and then it goes up to a constant value. At $t=0.5 \mathrm{~s}$, all the material particles except B13, C14, and K22 move downward toward the bottom of the weld, as can be seen in Fig.5-10(c), and the farther material particle away from the pin is, the further it goes toward the weld bottom. The reasons for the flow behaviors of particle $\mathrm{B} 13, \mathrm{C} 14$ and $\mathrm{K} 22$ refer to $\mathrm{T} 2, \mathrm{~T} 4$, and $\mathrm{T} 11$. 


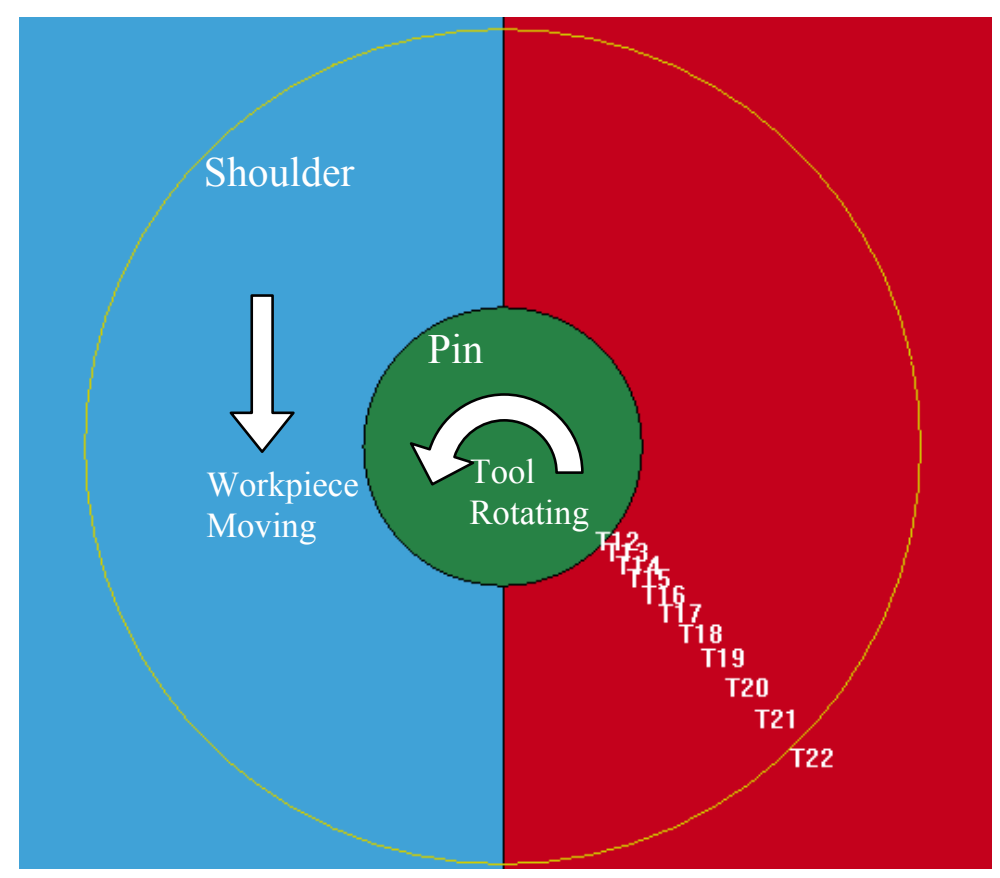

(a) Initial Positions

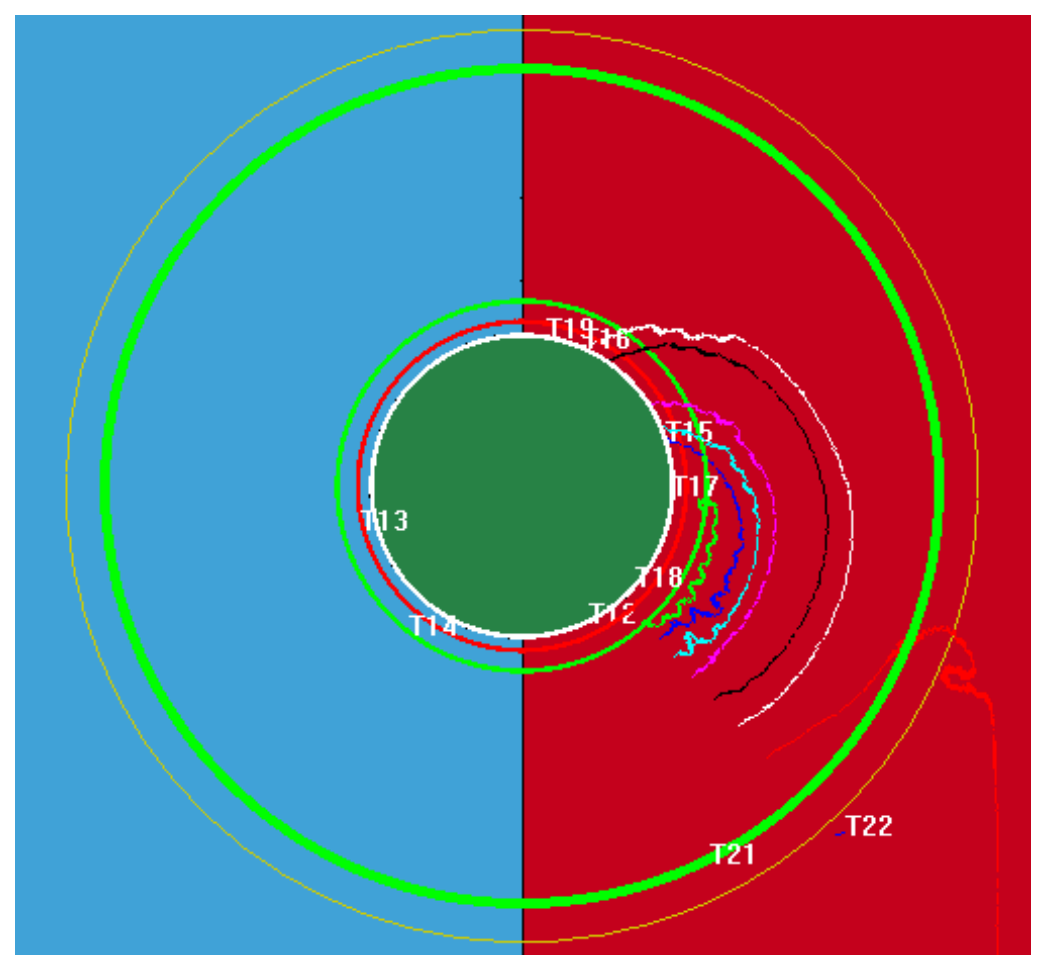

(b) Planar Positions at $t=0.5 \mathrm{~s}$ 


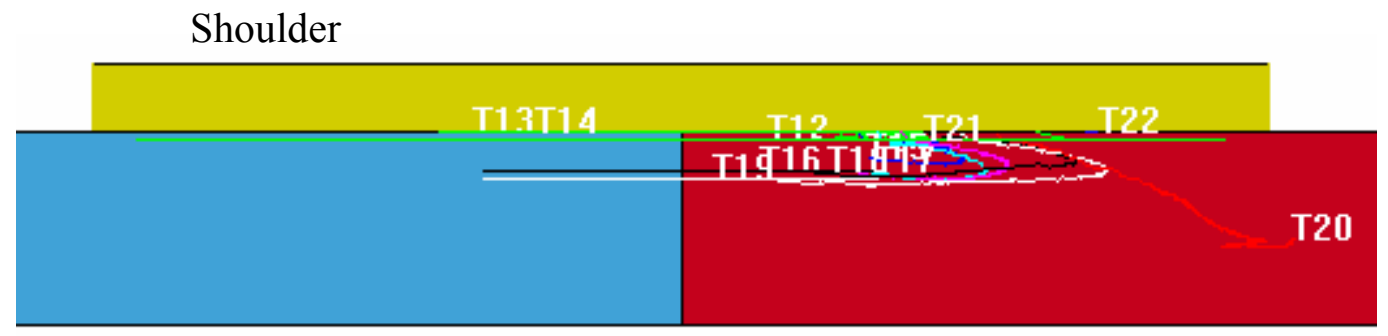

(c) Vertical (Depth-wise) Positions at $t=0.5 \mathrm{~s}$

Figure 5-10 Planar and Vertical (Depth-wise) Motions of Tracer T12-T22 in the Model

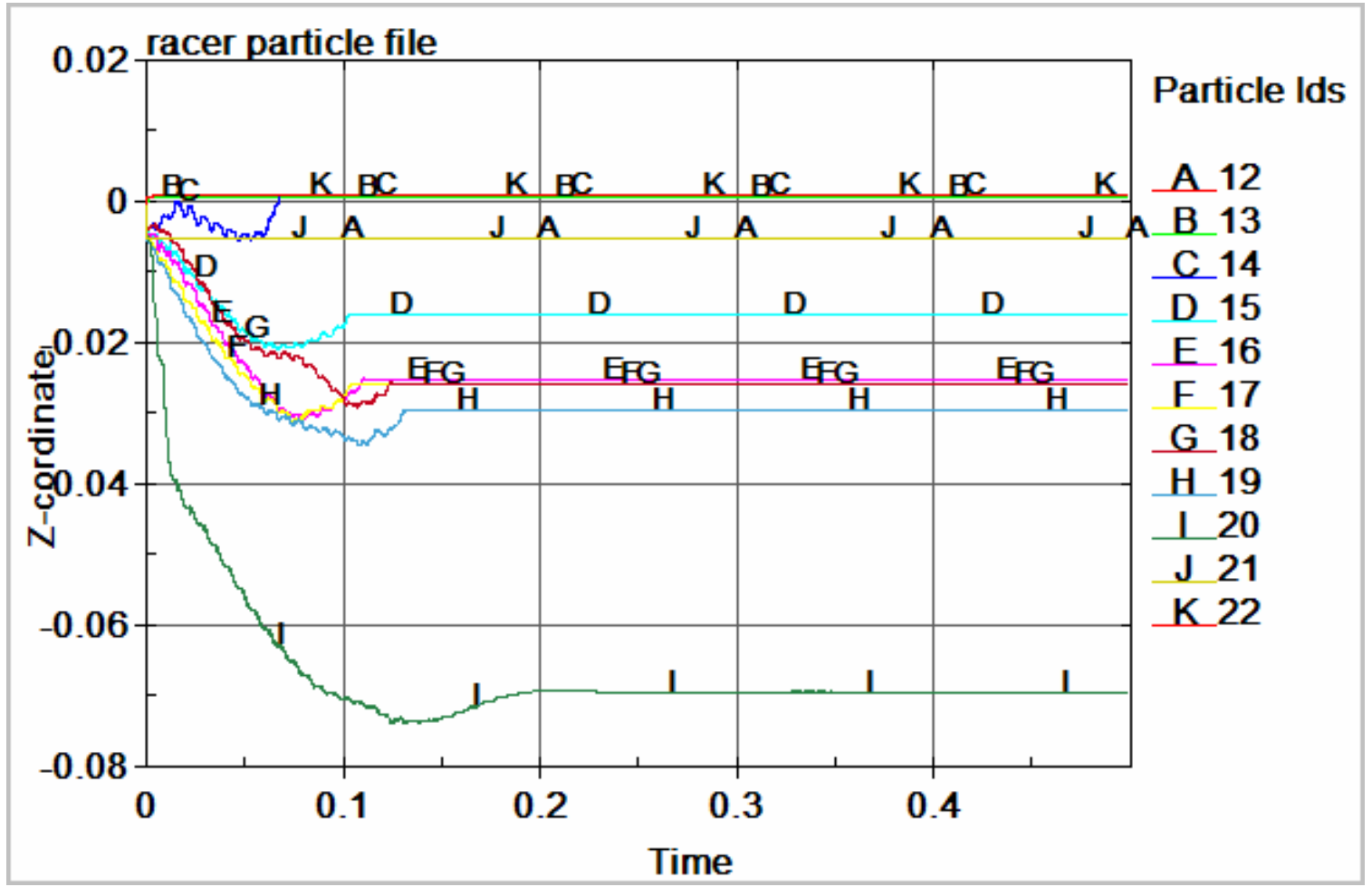

Figure 5-11 Z Coordinates vs. Time for Tracer T12-T22 
Again, the same method is applied to analyzing the material flow patterns of Tracer T23-33. The initial positions of tracer T23-T33 in the model are shown in Fig.512(a) and their planar and vertical positions at $t=0.5 s$ are plotted in Fig.5-12 (b) and (c), respectively. Z coordinates for those tracers over time are plotted in Fig.5-13. Particle A23-K33 represent tracer T23-T33, respectively.

In Fig.5-12(b), it is shown that, in the beginning of FSW, the material particles initially located at the retreating side move around the pin to the advancing side in the rotational direction of the tool. After $t=0.5 s$, particle T23, T28, T30, and T31 move to the advancing side and particle T24, T25, T26, T27, T29, T32 and T33 are still on the retreating side. During FSW, particle T23, T25, T30, T31, T33 stop moving when they arrive at some place while others keep rotating with the welding tool since they get on the welding tool.

In Fig.5-13, it is shown that at $t=0.5 \mathrm{~s}$, all the material particles move downward toward the bottom of the weld except material particle B24, D26, E27 and K33, as can be seen in Fig.5-12(c). The reasons for the flow behavior of B24, D26, E27 and K33 refer to $\mathrm{T} 2, \mathrm{~T} 4$, and $\mathrm{T} 11$. 


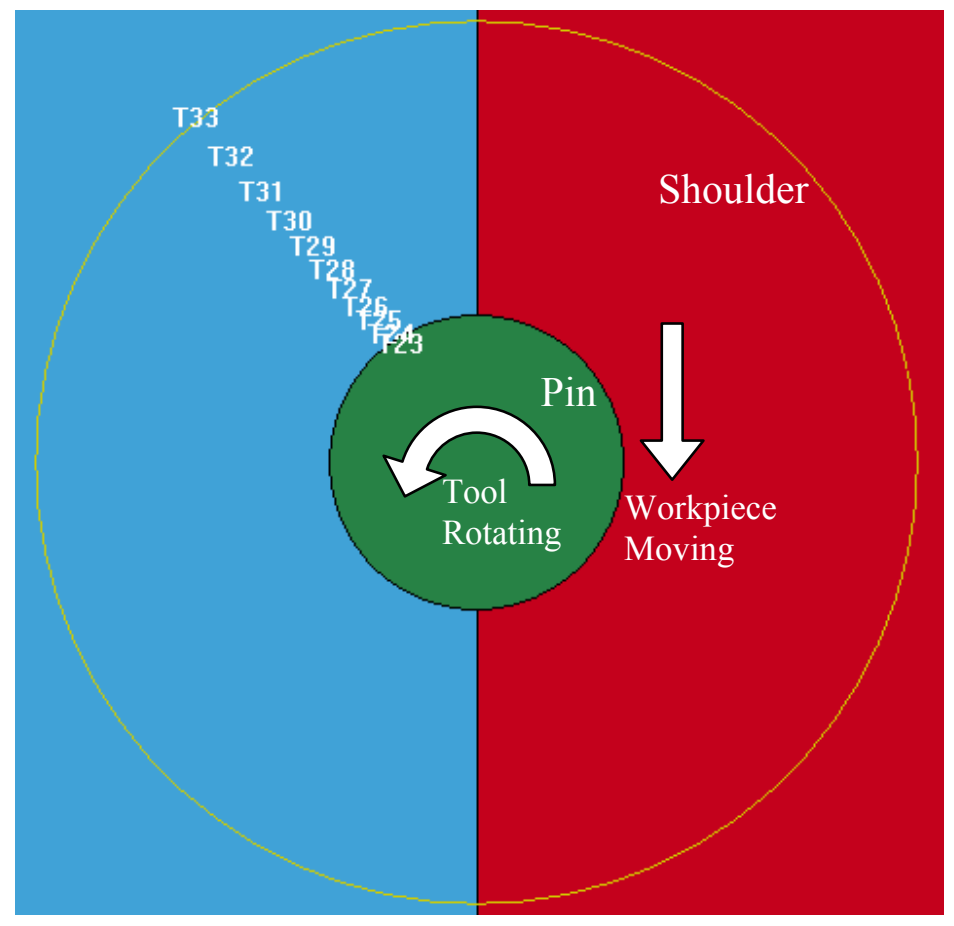

(a) Initial Positions

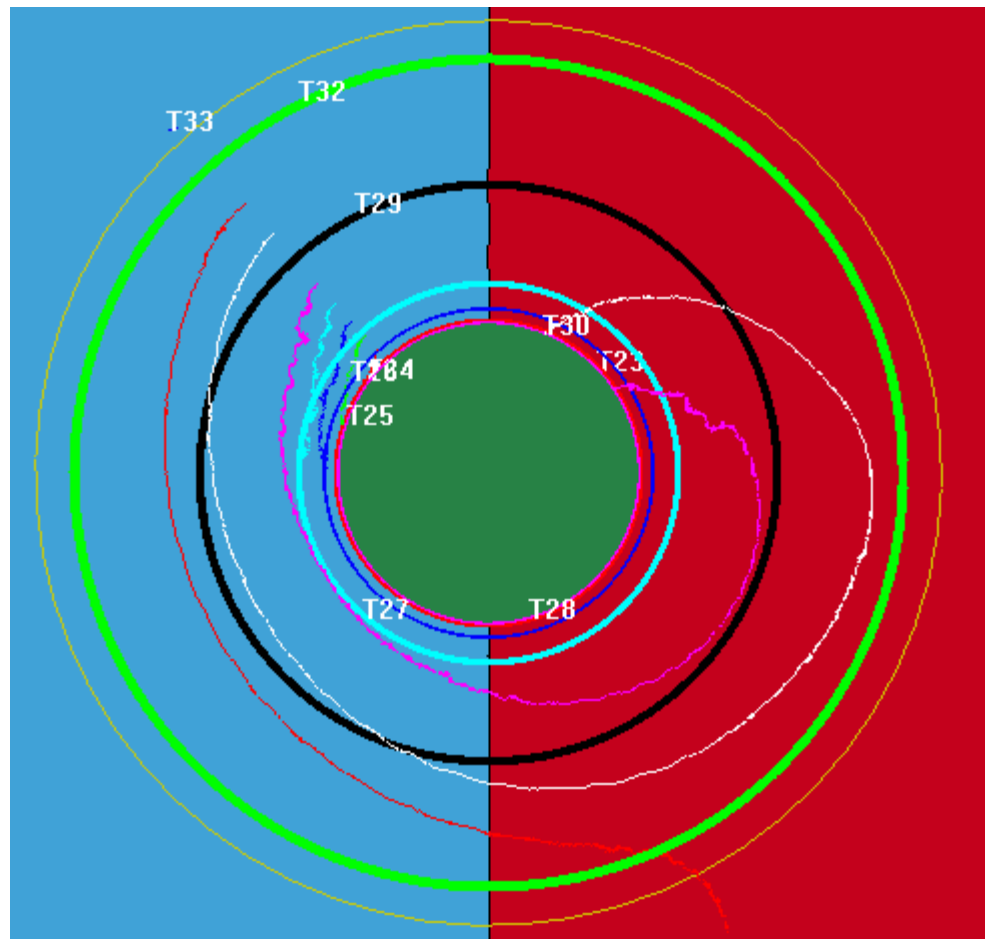

(b) Planar Positions at $t=0.5 \mathrm{~s}$ 


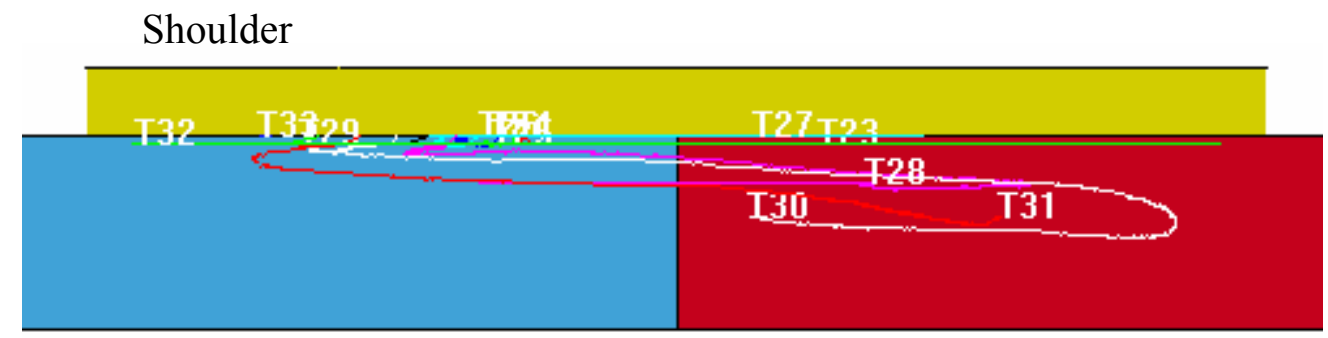

(c) Vertical (Depth-wise) Positions at $t=0.5 \mathrm{~s}$

Figure 5-12 Planar and Vertical (Depth-wise) Motions of Tracer T23-T33 in the Model

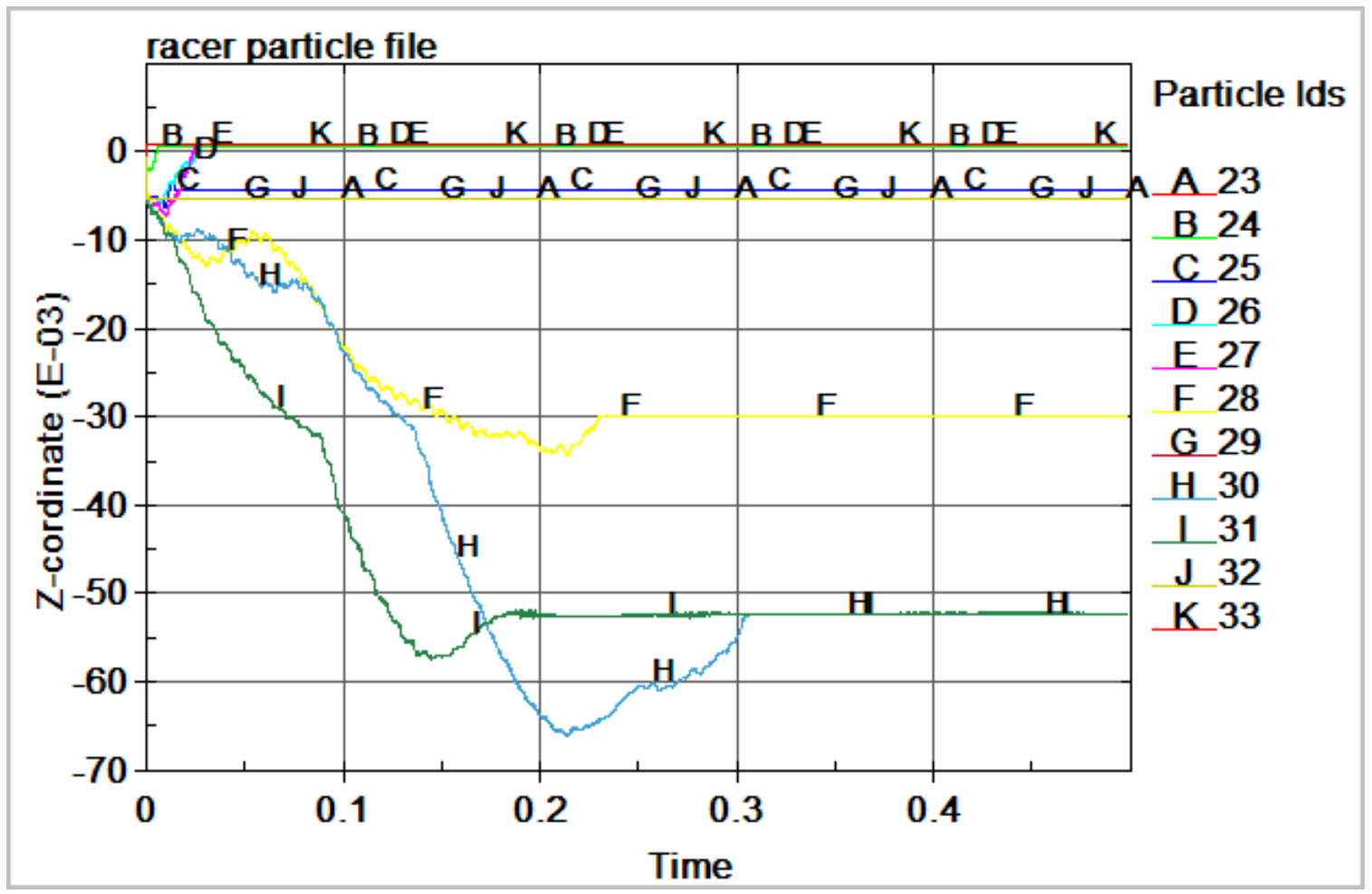

Figure 5-13 Z Coordinates vs. Time for Tracer T23-T33 
Once again, the material flows of T34-44 are analyzed using the same method above. The initial positions of tracer T34-T44 in the model are shown in Fig.5-14(a) and their planar and vertical positions at $t=0.5 \mathrm{~s}$ are plotted in Fig.5-14(b) and (c), respectively. $\mathrm{Z}$ coordinates for those tracers over time are plotted in Fig.5-15. Particle A34-K44 represent tracer T34-T44, respectively.

In Fig.5-14(b), again, it is shown that at the beginning of FSW, the material particles initially located at the retreating side are pushed around the pin to the advancing side in the rotational direction of the tool. After $t=0.5 \mathrm{~s}$, particle T39, T40, T41 go to the advancing side and others remains on the retreating side. During FSW, particle T40, T41, T44 stop moving after they get to a place while others keeps rotating with the welding tool as they get on the pin or the shoulder.

In Fig.5-15, it is shown that at $t=0.5 \mathrm{~s}$, all the material particles move downward toward the bottom of the weld except material particle B35 and K44, as can be seen in Fig.5-14(c). The reasons for B35 and K44 refer to T2, T4 and T11. 


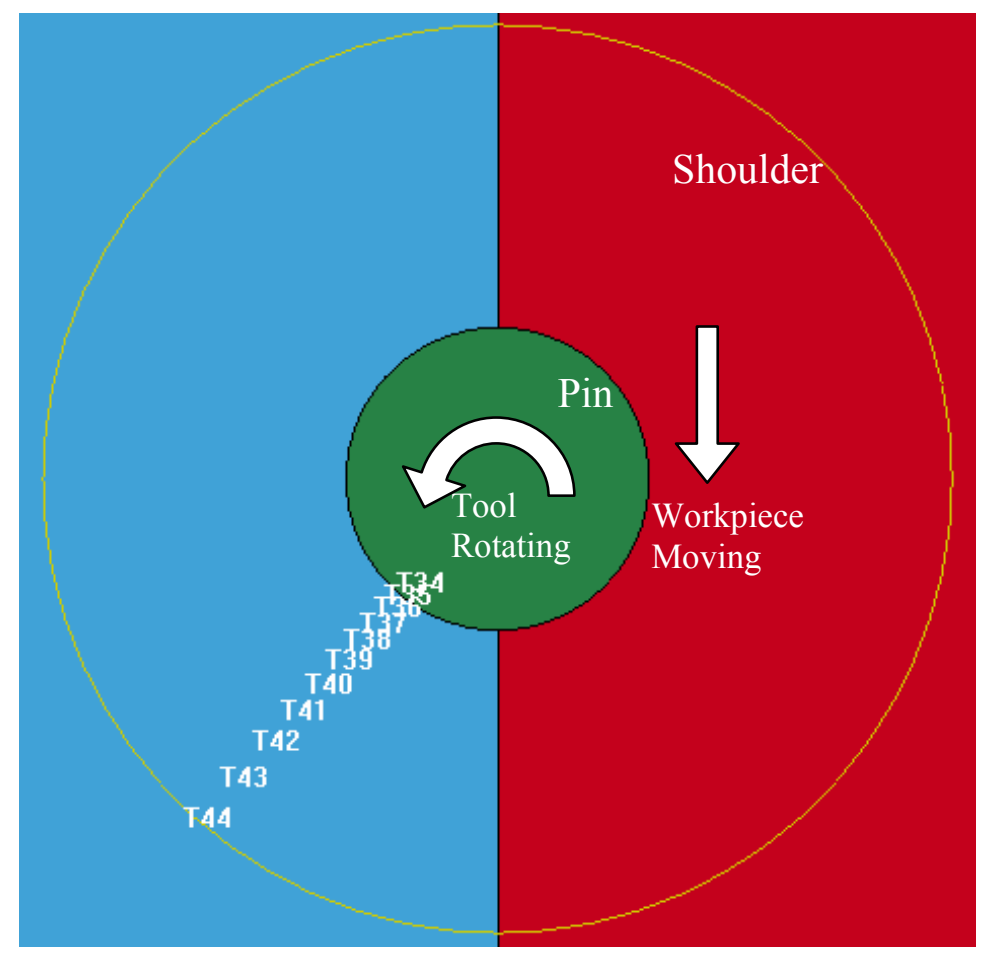

(a) Initial Positions

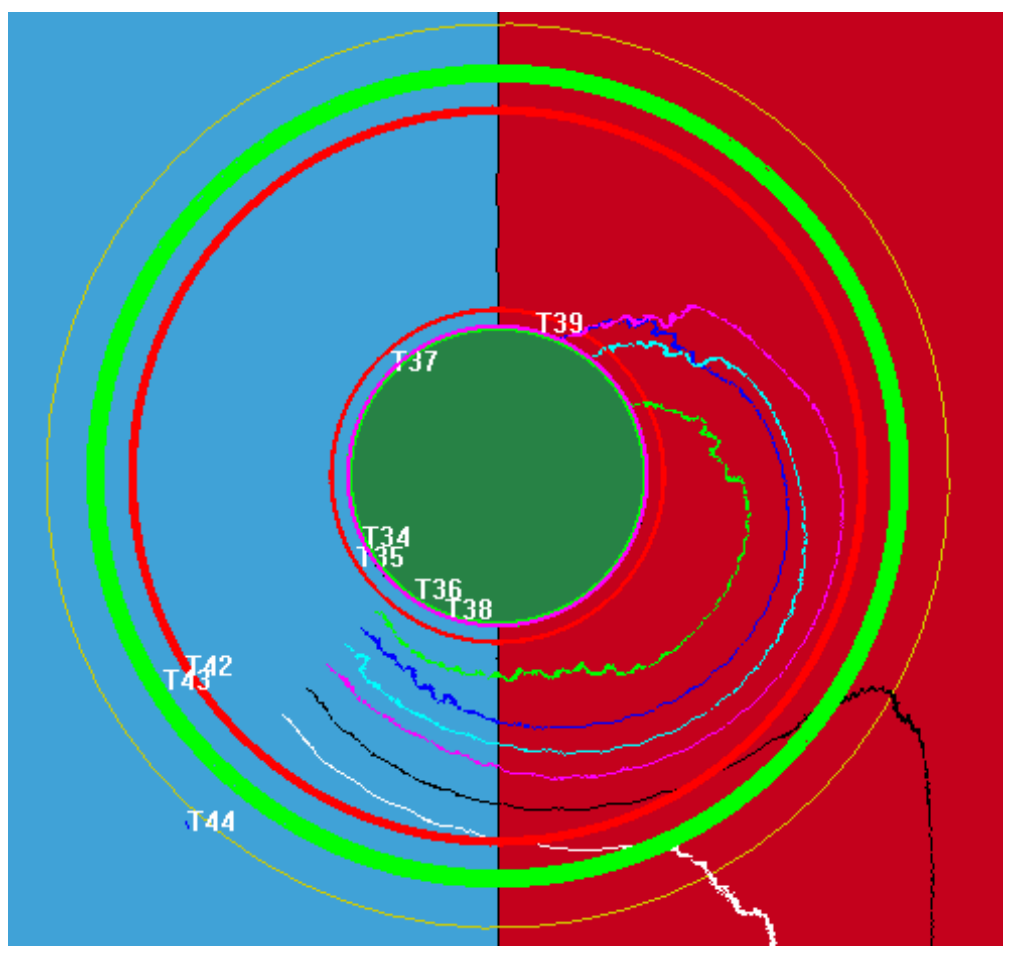

(b) Planar Positions at $t=0.5 \mathrm{~s}$ 


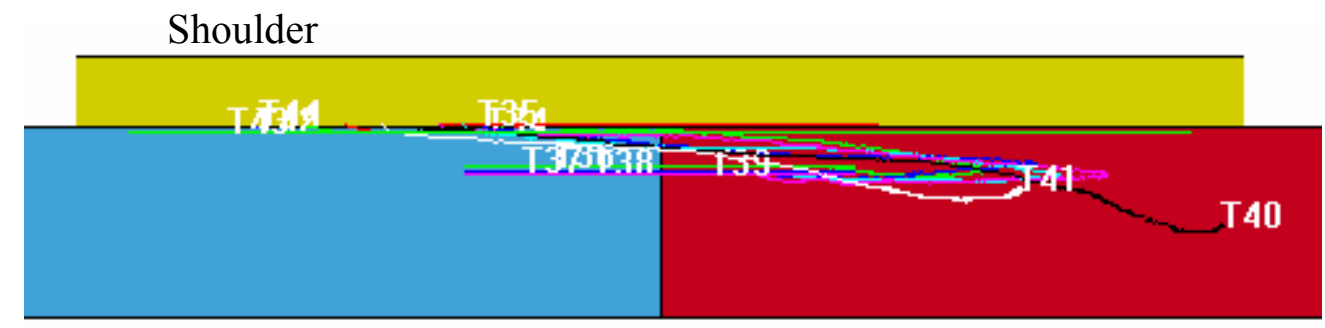

(c) Vertical Positions at $t=0.5 \mathrm{~s}$

Figure 5-14 Planar and Vertical (Depth-wise) Motions of Tracer T34-T44 in the Model

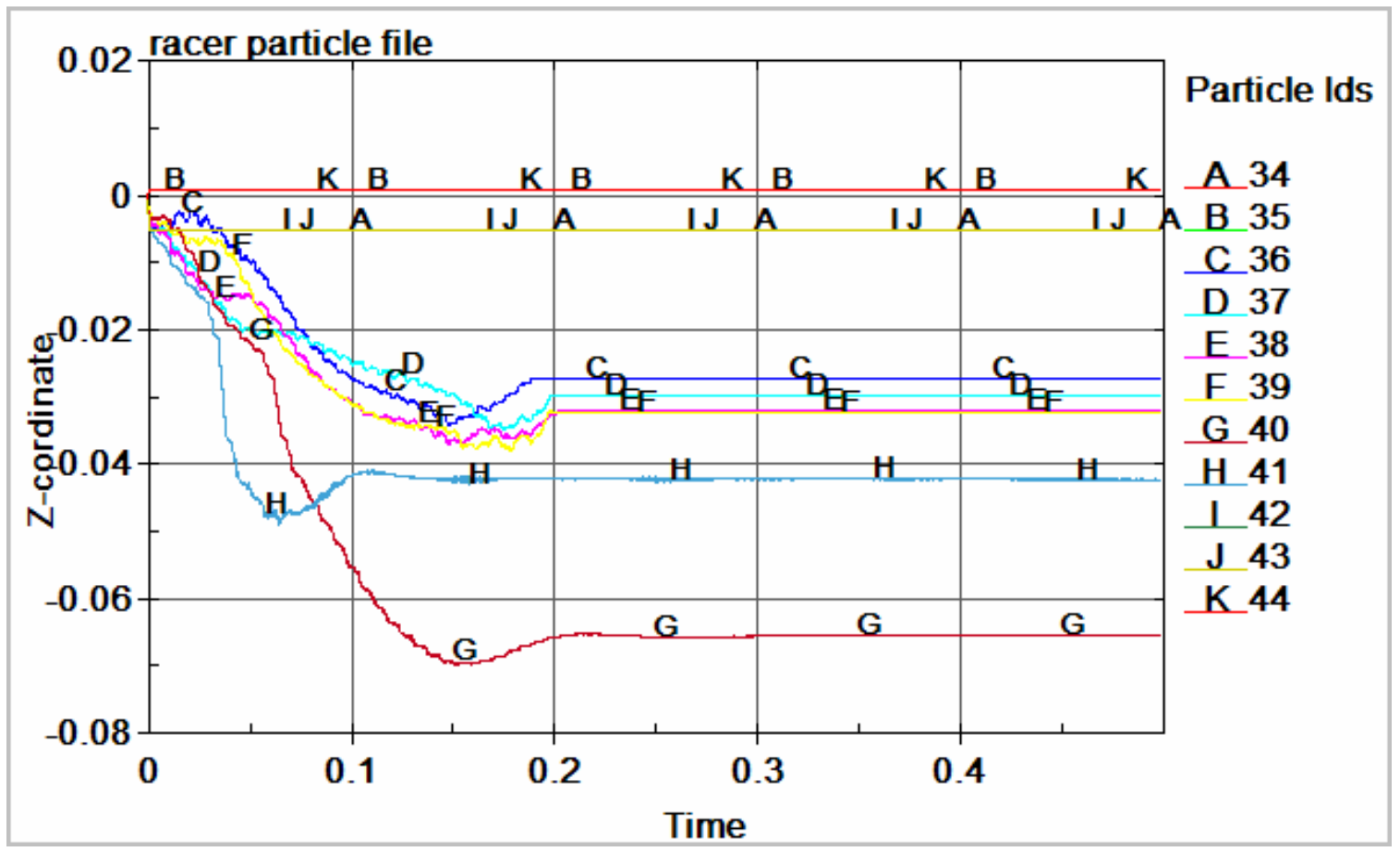

Figure 5-15 Z Coordinates vs. Time for Tracer T34-T44 


\subsubsection{Comparison of Model Flow Patterns with Experimental Data}

Because of the lack of experimental data, quantitative validation of simulation results for material flow characteristics in FSW is currently not possible. But a qualitative comparison can be made between the material flow patterns predicted by the finite element models with the patterns from the published test conducted by Seidel et al. [5253]. In the test, the material flow in friction stir welds was visualized using a marker insert technique, as discussed in chapter 2. The workpieces were made of AA2195-T8 and had a thickness of $8.1 \mathrm{~mm}$. The pin was threaded and had a diameter of $9.9 \mathrm{~mm}$, a length of $7.9 \mathrm{~mm}$. The shoulder had a diameter of $25.4 \mathrm{~mm}$. The tool advance per rotation (welding speed/rotational speed) was 0.61 . The markers used in the test were AA5454H32 sheets and were placed on the advancing and retreating side, respectively, at three different heights: top, middle and bottom, as shown in Fig.5-16.

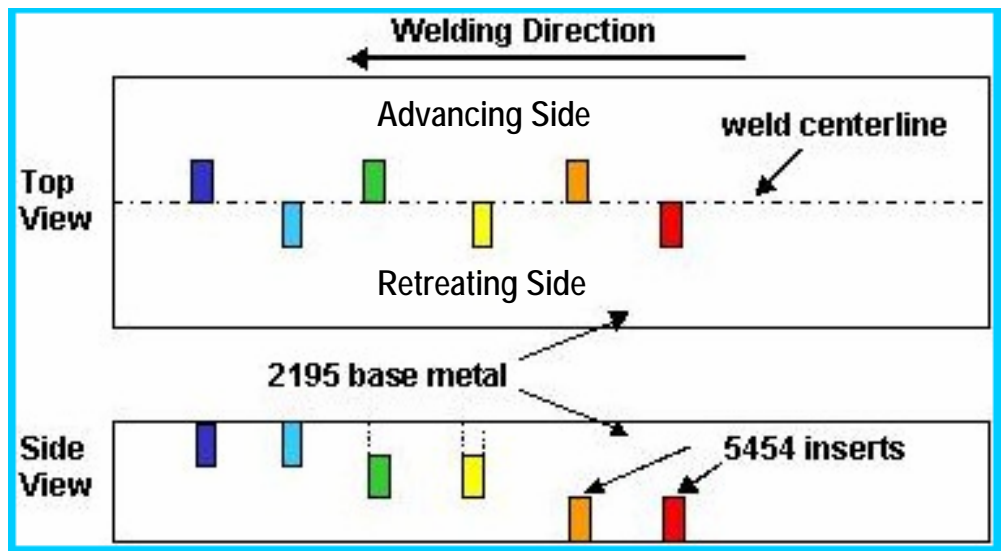

Figure 5-16 Schematic Drawing of the Marker Insert Placement (Seidel [53]) 
Fig.5-18 shows the final positions of the markers at the top of the weld after the welding tool pass through. Actually, this figure was formed by combining the advancing and retreating side markers at the top of weld into one image. In the figure, the black shades are the base material and the white shades are the markers. The retreating side is on the left side of the figure and the advancing side is on the right. It is seen that the material on the retreating side are transported along with the rotating tool to the advancing side. It is also shown that the material on the retreating side tends to move close to the shoulder edge along the workpiece moving direction. While the material on the advancing side moves closer to the pin against the workpiece moving direction.

For the purpose of comparison, the material flow pattern predicted by the current model is plotted in Fig.5-17. Fig.5-17(a) show the initial positions of the tracer particles which are originally aligned along the straight line perpendicular to the weld line, at the top surface of the workpieces. Fig.5-17(b) shows their positions at $t=0.5 \mathrm{~s}$. It is also shown that the material particles move from the retreating side to the advancing side by the rotation of the welding tool. The particles on the retreating side move in a larger circumferential manner while the particles on the advancing side move toward the pin. These flow patterns are consistent with the results from the experimental observations in Fig.18.

Fig.5-19 shows the vertical motions of the markers inside the workpiece material (depth-wise). The images were generated by projecting the marker positions onto a vertical plane in the welding direction. The left and right image show the marker positions before and after welding, respectively. The dashed lines denote the pin diameter. As can be seen, the tracing markers are pushed downwards by the welding tool. 
Then these markers are moved upwards since the material flow path is restricted in $\mathrm{x}-\mathrm{y}-\mathrm{z}-$ direction by the undeformed base material, the shoulder and pin, and the backing plate. The upward motion is strong around the pin. These vertical flow patterns also support the simulation results shown in section 5.2.1.2

It is noted that in the comparison above, different workpiece material, different geometries, and different tool advance per rotation are used in the finite element model and the published test. The reason why aluminum alloy 6061-T6 is chosen for the current model is because its detail material properties are available in the literature, which are important for successfully modeling of FSW. However so far no one published FSW test with the same material has the marker position measurements, a quantitative validation of the present numerical simulation results cannot be performed. But, if the marker positions shown in Figs.5-18 and 5-19 representing a common material pattern in FSW is accepted, a qualitative comparison of the simulation results with the test data can then be made. Actually, the comparison made above has been shown that the finite element model is qualitatively supported by the test. 


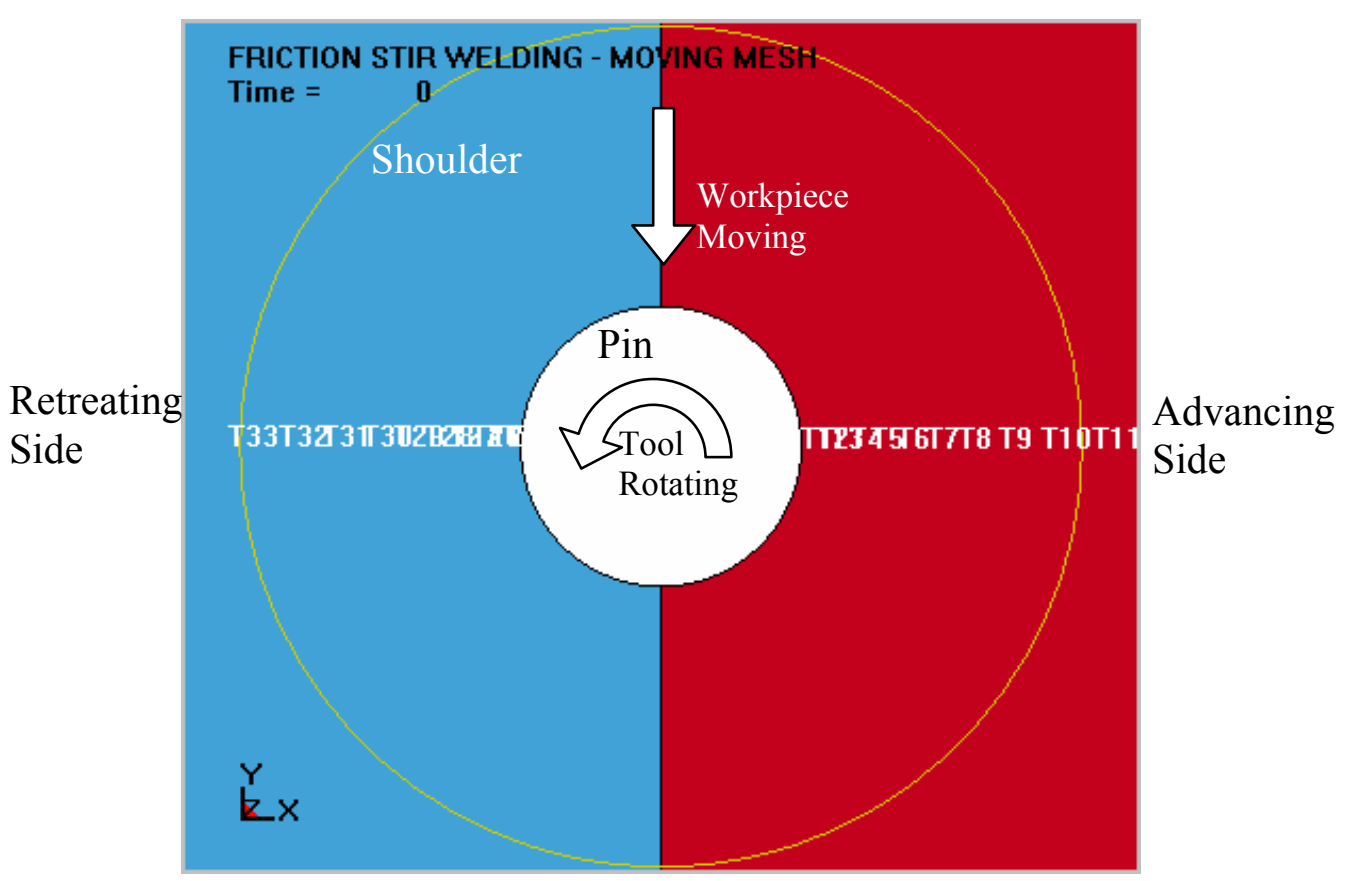

(a) Initial Positions of Tracer Particles

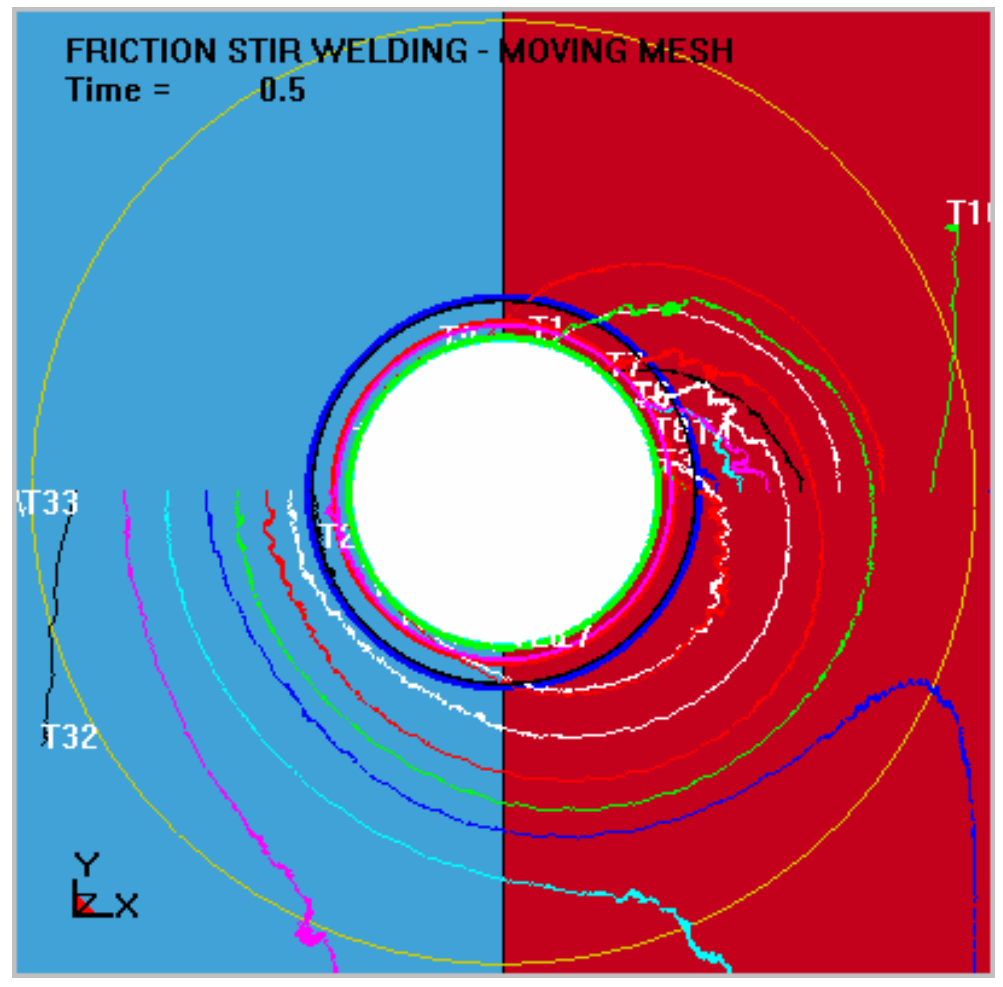

(b) Tracer Particle Positions at $t=0.5 \mathrm{~s}$

Figure 5-17 Finite Element Predictions of the Material Flow Pattern on the Top of Surface 


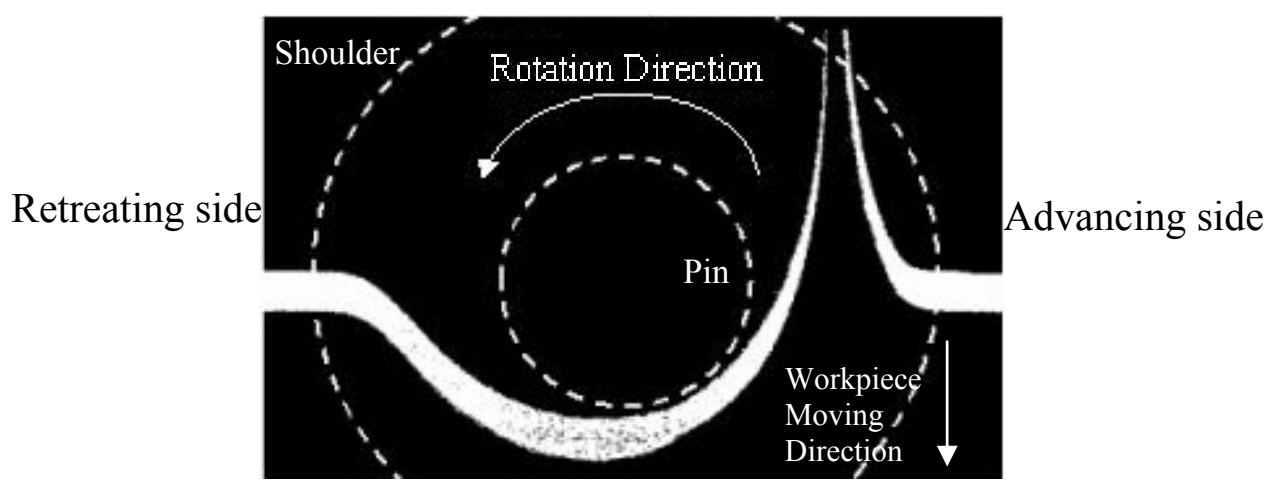

Figure 5-18 Material Motion on the Top Surface of the Weld in FSW Test (Seidel [53])

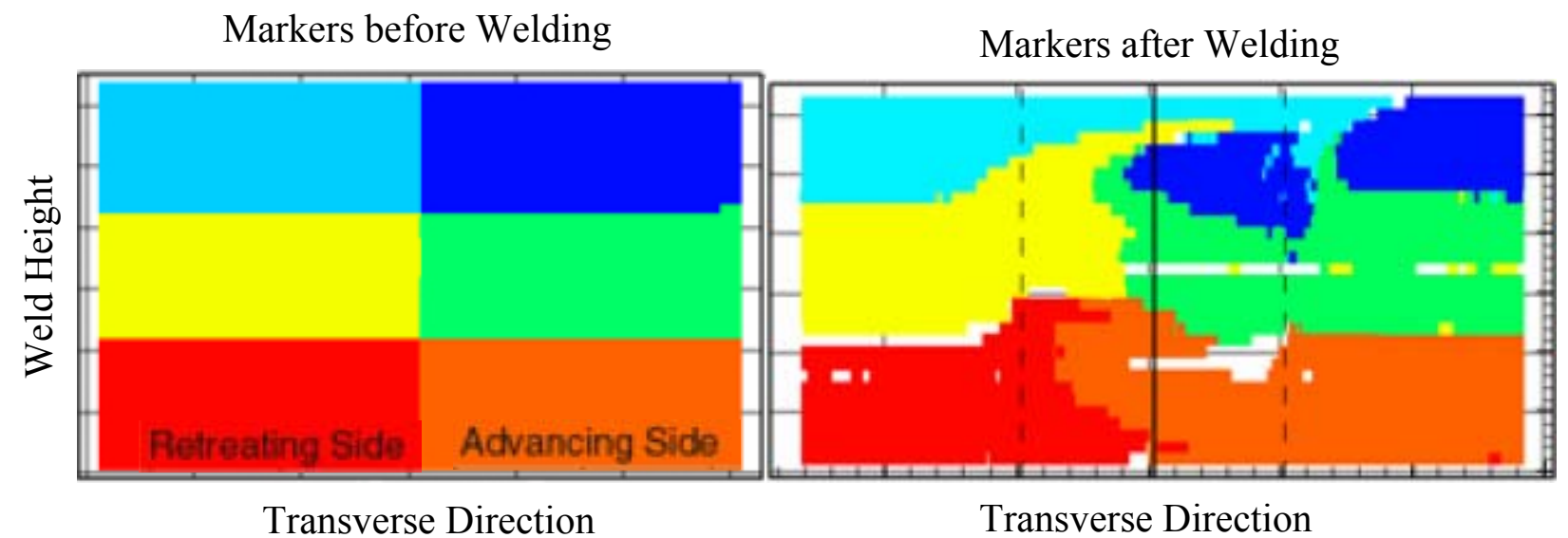

Figure 5-19 Vertical Material Motion (Depth-wise) in FSW Test (Seidel [53]) 


\subsubsection{Summary}

In this case, the material flow in the friction stir butt weld of the same aluminum alloy is characterized based on the volume fraction function and the tracer particle technique. By the use of the tracers in the model, it is directly observed how a material particle moves around the welding tool as the process proceeds.

The simulation results show that the material flow is not symmetric about the joint line. The flow patterns on the advancing side and retreating sides are different.

By the rotation of the welding tool, the material in the advancing side moves around the pin to the retreating side in the rotational direction of the tool. And the material in the retreating side is also transported around the pin to the advancing side in the rotational direction of the tool. During FSW, some material particles are carried more than once around the rotating pin. In addition, the forging action combined the stir action of the tool produces a secondary, down-and-up motion of material under the welding tool during FSW.

The simulation predictions of the planar and vertical (depth-wise) motions of the workpiece material are consistent with the published experimental results. 


\subsubsection{Case Two - The Material Flow in Two Material Models}

\subsubsection{Problem Definition}

In this case, the material behavior of the workpieces is modeled as power law isotropic plasticity, whereas everything else remains same as the first case. The geometries of the workpieces, the pin and shoulder and all the reference values are listed in Table 5-4.

The power law isotropic plastic material model has been implemented into LSDYNA code. It provides elastoplastic behavior with isotropic hardening. The yield stress is a function of plastic strain and obeys the equation:

$$
\sigma_{y}=k \varepsilon^{n}=k\left(\varepsilon_{y p}+\bar{\varepsilon}^{p}\right)^{n}
$$

where $\varepsilon_{y p}$ is the elastic strain to yield and $\bar{\varepsilon}^{p}$ is the effective plastic strain (logarithmic)

A parameter, SIGY, in the input governs how the strain to yield is identified. If SIGY is set to zero, the strain to yield is found by solving for the intersection of the linearly elastic loading equation with the strain hardening equation

$$
\begin{gathered}
\sigma=E \varepsilon \\
\sigma=k \varepsilon^{n}
\end{gathered}
$$

which gives the elastic strain at yield as

$$
\varepsilon_{y p}=\left(\frac{E}{k}\right)^{\frac{1}{n-1}}
$$

If SIGY yield is non-zero and greater than 0.02 then

$$
\varepsilon_{y p}=\left(\frac{\sigma_{y}}{k}\right)^{\frac{1}{n}}
$$


Strain rate is accounted for using the Cowper and Symonds model which scales the yield stress with the factor $1+\left(\frac{\dot{\varepsilon}}{C}\right)^{\frac{1}{P}}$, where $\dot{\varepsilon}$ is the strain rate. A fully viscoplastic formulation is optional with this model which incorporates the Cowper and Symonds formulation within the yield surface. An additional cost is incurred but the improvement in results can be dramatic.

In this case, the material parameters required for the workpieces are mass density, Young's modulus, Poisson's ratio, strength coefficient and hardening exponent. The values for those parameters are listed in Table 5-5. Again, the pin and shoulder are assumed as rigid bodies.

Table 5-4 Geometries and Reference Values

\begin{tabular}{c|c|c|c}
\hline & Workpieces & Pin & Shoulder \\
\hline \hline Diameter (in) & 3.0 & 0.2552 & 0.7656 \\
\hline Thickness (in) & 0.125 & 0.12 & 0.05 \\
\hline Translational Velocity & \multicolumn{3}{|c}{$0.2 \mathrm{in} / \mathrm{sec}$} \\
\hline Rotational Velocity & \multicolumn{3}{|c}{$94.2 \mathrm{rad} / \mathrm{sec}(900 \mathrm{rpm})$} \\
\hline Frictional Coefficient & \multicolumn{3}{|c}{0.47} \\
\hline Forging & \multicolumn{3}{|c}{$0.005 \mathrm{in}$} \\
"Force"(Displacement) & \multicolumn{3}{|c}{}
\end{tabular}

Table 5-5 Material Parameters

\begin{tabular}{c|c|c|c|c|c}
\hline Material & $\begin{array}{c}\text { Mass Density } \\
\left(\left(\mathrm{l}^{*} \mathrm{~s}^{\wedge} \text { 2)/in^4) }\right.\right.\end{array}$ & $\begin{array}{c}\text { Young's } \\
\text { Modulus } E \\
(\mathrm{psi})\end{array}$ & $\begin{array}{c}\text { Poisson's } \\
\text { Ratio }\end{array}$ & $\begin{array}{c}\text { Strength } \\
\text { coefficient } \\
(\mathrm{psi})\end{array}$ & $\begin{array}{c}\text { Hardening } \\
\text { Exponent } \\
\mathrm{n}\end{array}$ \\
\hline $\begin{array}{c}\text { Workpieces } \\
(\mathrm{Al} \text { 6061-T6) }\end{array}$ & 0.00025 & $1.0 \mathrm{e} 7$ & 0.33 & 60100 & 0.0742 \\
\hline $\begin{array}{c}\text { Pin and } \\
\text { shoulder } \\
\text { (Steel) }\end{array}$ & 0.00073 & $3.0 \mathrm{e} 7$ & 0.28 & --- & --- \\
\hline
\end{tabular}




\subsubsection{Simulation Results}

The volume fraction contours for the workpieces with the power law isotropic plastic model at $t=0.2 \mathrm{~s}$ are plotted in Fig.5-20 (a). The left image shows the material flow in the retreating side of the friction stir weld while the right image shows the material flow in the advancing side. In Fig.5-20, a comparison of the volume fraction contours at $t=0.2 \mathrm{~s}$ is also made between the power law isotropic plastic model (see Fig.5-18(a)) and the elastic-perfectly plastic with kinematic hardening model (see Fig.518(b)). It can be seen that the material flow patterns in the retreating and advancing sides are different in these two kinds of material models. The material flow is smaller in the power law isotropic plastic model than the elastic-perfectly plastic with kinematic hardening model.

The plastic strains in the two workpieces with two different material models are quantitatively compared by plotting the histories of maximum of effective plastic strain, as shown in Fig.5-21. The red curve with letter A represents the maxima of the effective plastic strain in material 1 (advancing side) and the green curve with letter B corresponding to material 2 (retreating side). From the figure, before $t=0.15 \mathrm{~s}$, the maximum of the effective plastic strain increases with time for both models, but the maximum of the effective plastic strain is smaller in the power law isotropic plastic model than the elastic-perfectly plastic with kinematic hardening model at same time instance. After $t=0.15 \mathrm{~s}$, the maximum of the effective plastic strain in the power plastic model continues to increase with time. However, the elastic-perfectly plastic with kinematic hardening model shows different pattern: the maximum of the effective plastic 
strain in material 1 starts to decrease then increases, while the maximum of the effective plastic strain in material 2 is almost constant.

This case shows the material flow patterns are different in both material models. It is also shown that the different material models can be easily incorporated into the finite element models to investigate their effects on the material flow in the friction stir weld. With the increase of the number of the materials that can be welded by the FSW technique, modeling of the FSW process will take its place as a major development tool alongside the experimental work, reducing the amount of time and resources required for full experimental trials. 

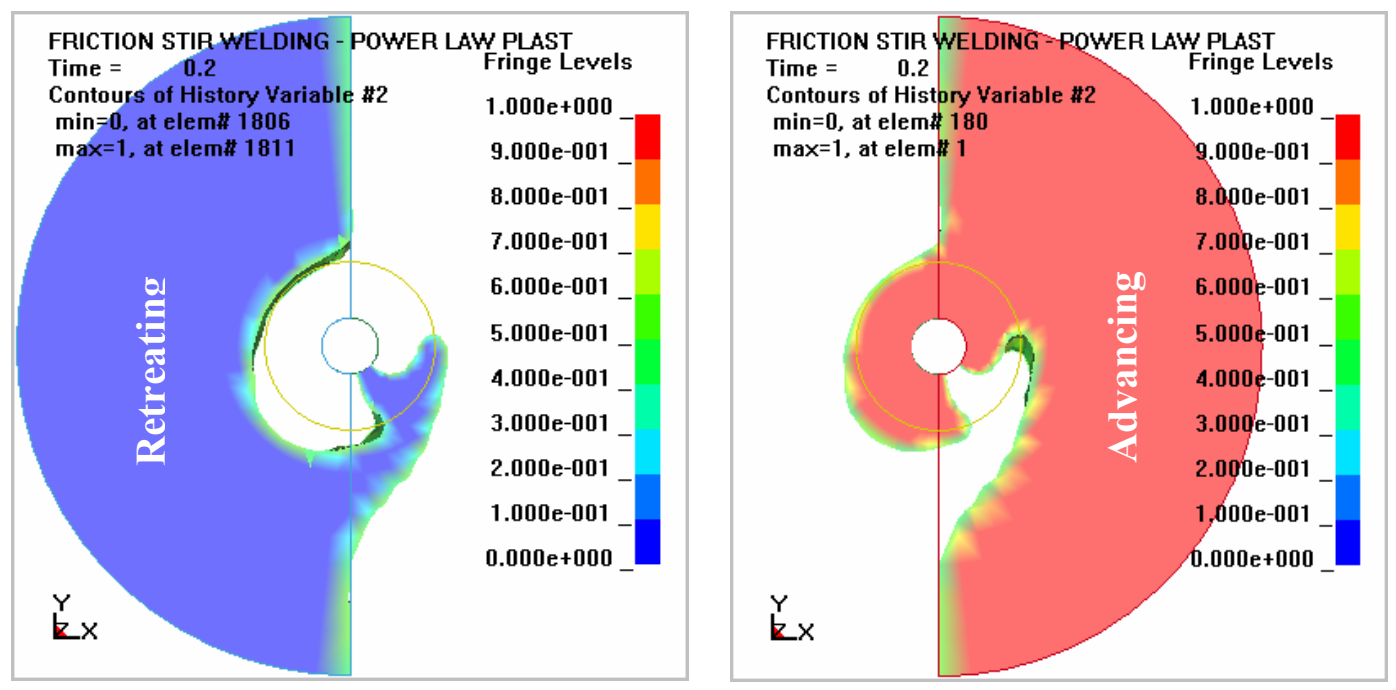

(a) The Power Law Isotropic Plastic Model
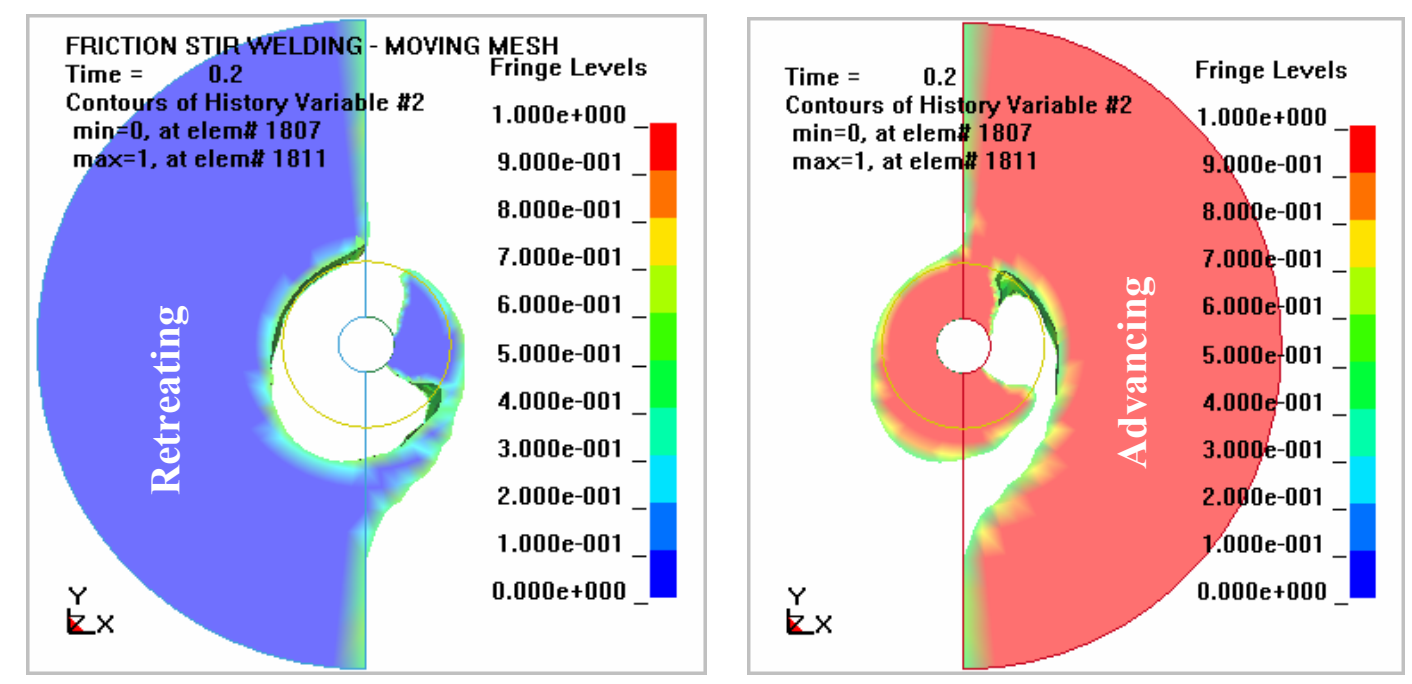

(b) The Elastic-perfectly Plastic with Kinematic Hardening Model

Figure 5-20 Comparison of Volume Fraction Contours between Two Different Material Models at $t=0.2 \mathrm{~s}$ 


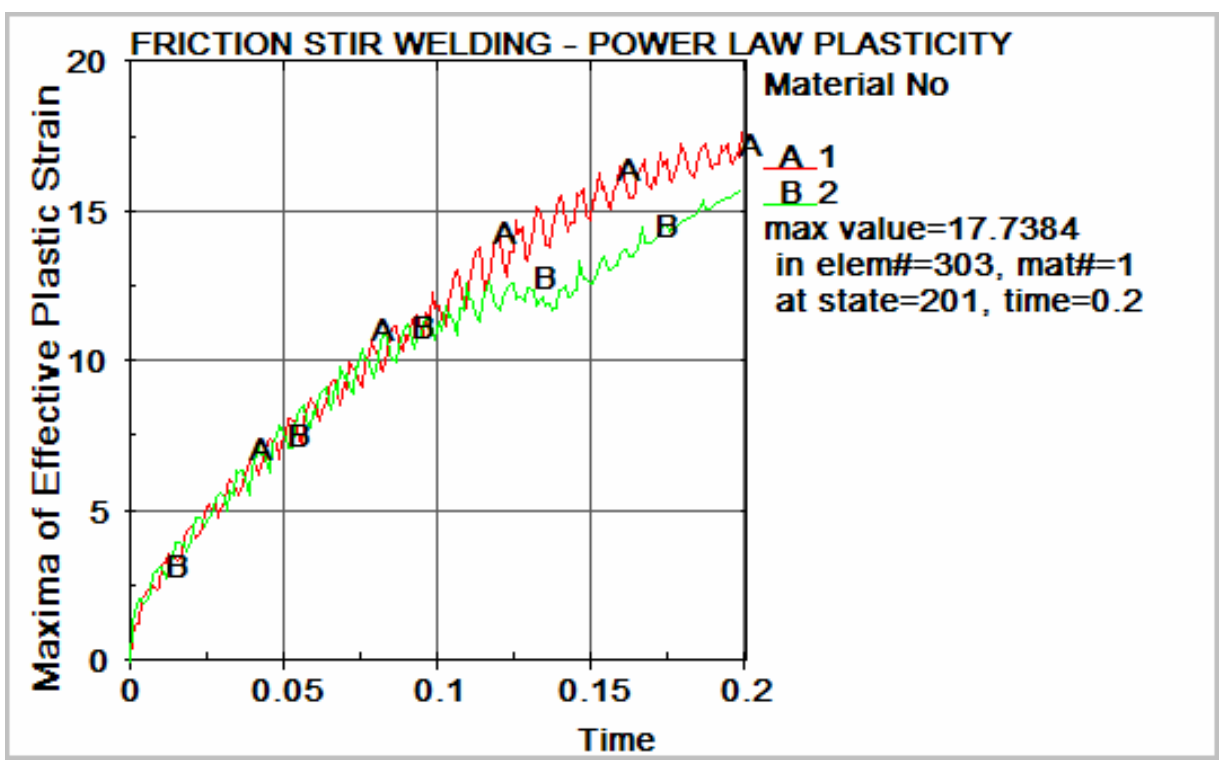

(a) The Power Law Isotropic Plastic Model

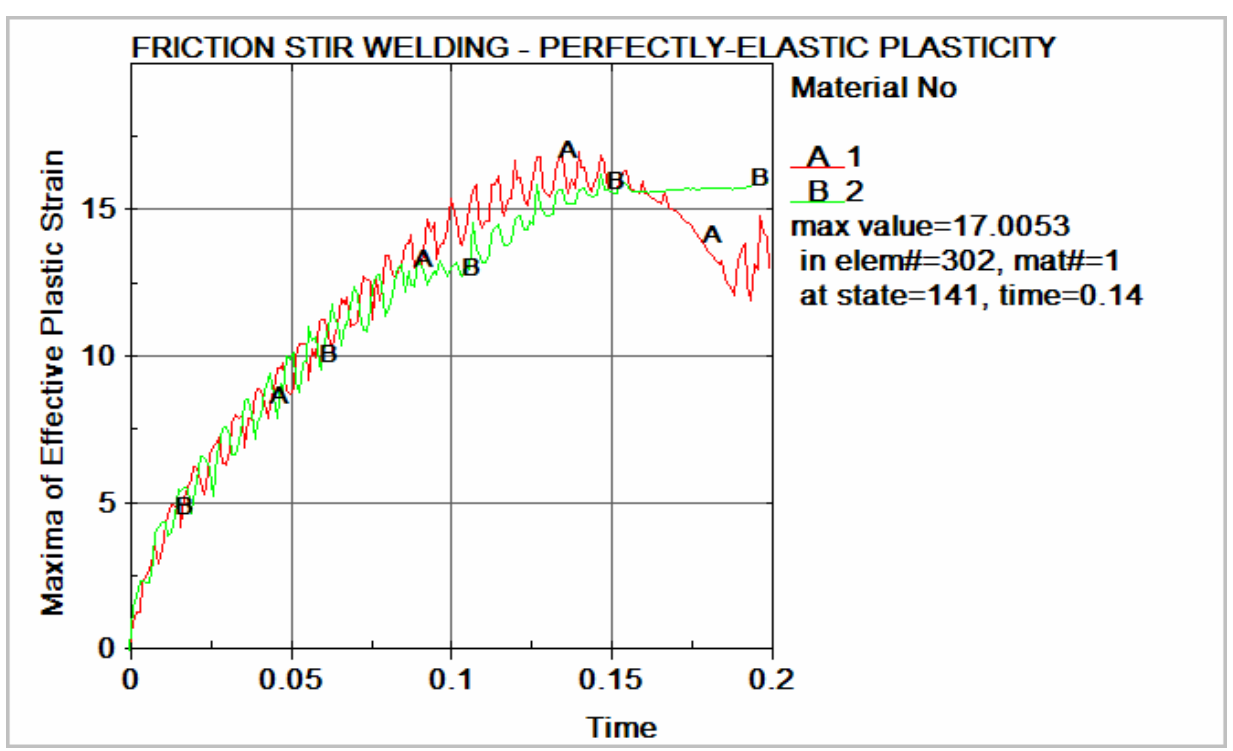

(b) The Elastic-Perfectly Plastic with Kinematic Hardening Model

Figure 5-21 Comparison of the Maximum Effective Plastic Strain between Two Different Material Models throughout the Computation 


\subsubsection{Case Three - The Material flow in the Dissimilar Aluminum Alloy Weld}

The material flow behavior in the dissimilar friction stir welds is quite complex. The FSW of a wide variety of the both the same and dissimilar aluminum alloys to one another has been shown to involve dynamic recrystallization as the mechanism to accommodate the superplastic deformation that facilitates the bond. Complex, fluid -like flow patterns often arise as a result of irregular intercalation lamellae formed by the flow of one recrystallized regime within or over another (Ouyang et al. [44] and Ying et al. [62]). Normally, during FSW, each one of two workpieces with different properties is placed at the specified (advancing or retreating) side of a friction stir weld and interchanging their positions causes a bad weld.

This case simulates the FSW of Al 6061-T6 alloy to Al 2024-T6 alloy using the moving mesh method. The material flow behavior in the dissimilar weld is of interest.

\subsubsection{Problem Definition}

The problem in the first case serves as a base for the simulation of the FSW of Al 6061-T6 alloy to Al 2024-T6 alloy. Problem definition is the same as the first case, as can be seen in Fig.5-4. The same geometries, material models, interface contact, loading and boundary conditions are taken except one workpiece is changed to Al 2024-T6 alloy. The geometries, all the reference values and material properties are listed in Tables 5-6 and 57. Again, the workpieces are treated as elastic-perfectly plasticity with kinematic hardening and the pin and shoulder are assumed rigid. 
Table 5-6 Geometries and Reference Values

\begin{tabular}{c|c|c|c}
\hline & Workpieces & Pin & Shoulder \\
\hline Diameter (in) & 3.0 & 0.2552 & 0.7656 \\
\hline Thickness (in) & 0.125 & 0.12 & 0.05 \\
\hline Translational Velocity & \multicolumn{3}{|c}{$0.2 \mathrm{in} / \mathrm{sec}$} \\
\hline Rotational Velocity & \multicolumn{3}{|c}{$94.2 \mathrm{rad} / \mathrm{sec}(900 \mathrm{rpm})$} \\
\hline Frictional Coefficient & \multicolumn{3}{|c}{0.47} \\
\hline Forging & \multicolumn{3}{|c}{$0.005 \mathrm{in}$} \\
"Force"(Displacement) & \multicolumn{3}{|c}{}
\end{tabular}

Table 5-7 Material Parameters

\begin{tabular}{c|c|c|c|c}
\hline Material & $\begin{array}{c}\text { Mass Density } \\
\left(\left(\mathrm{lb}^{*} \mathrm{~s}^{\wedge} 2\right) / \mathrm{in}^{\wedge} 4\right)\end{array}$ & $\begin{array}{c}\text { Young's } \\
\text { Modulus } E \\
(\mathrm{psi})\end{array}$ & $\begin{array}{c}\text { Poisson } \\
\text { Ratio }\end{array}$ & $\begin{array}{c}\text { Yield Stress } \\
\sigma_{y}(\mathrm{psi})\end{array}$ \\
\hline $\begin{array}{c}\text { Workpiece } \\
(\mathrm{Al} \text { 6061-T6) }\end{array}$ & 0.00025 & $1.0 \mathrm{e} 7$ & 0.33 & 39900 \\
\hline $\begin{array}{c}\text { Workpiece } \\
(\text { Al 2024-T6) }\end{array}$ & 0.00026 & $1.05 \mathrm{e} 7$ & 0.33 & 50000 \\
\hline $\begin{array}{c}\text { Pin and Shoulder } \\
\text { (Steel) }\end{array}$ & 0.00073 & $3.0 \mathrm{e} 7$ & 0.28 & --- \\
\hline
\end{tabular}

\subsubsection{Simulation Results}

The contours of history variable \#1 at $t=0 \mathrm{~s}, 0.04 \mathrm{~s}, 0.06 \mathrm{~s}, 0.08 \mathrm{~s}, 0.12 \mathrm{~s}, 0.16 \mathrm{~s}$, $0.2 s$ for the workpieces are shown in Figs.5-22(a)-(f), respectively. History variable \#1 is defined as the mass density of material. Fig.5-22(a) shows the initial mass density contour for the workpiece material. The red shades represent the mass density of the Al 2024-T6 alloy while the blue shades represent the mass density of the Al 6061-T6 alloy.

Based on the color changing in the workpiece material around the pin, the variation in the mass density of the workpiece material can be observed in Fig.5-22(b)-(f) 
as the process proceeds. Meanwhile, the mass density variation reflects the material flow pattern in the dissimilar friction stir weld. It is shown that the material flow exhibit distinctly asymmetric characteristics at both sides of the dissimilar weld. In addition, it is observed that the mass density in the lower portion is larger than that in the upper portion of the workpieces since the material moves from up to down, more mass is accumulated at the lower portion of the workpieces. But the mass for the entire model is still conservative.

In this case, it has been shown that the model has a capability to simulate the FSW process for joining the dissimilar aluminum alloys.

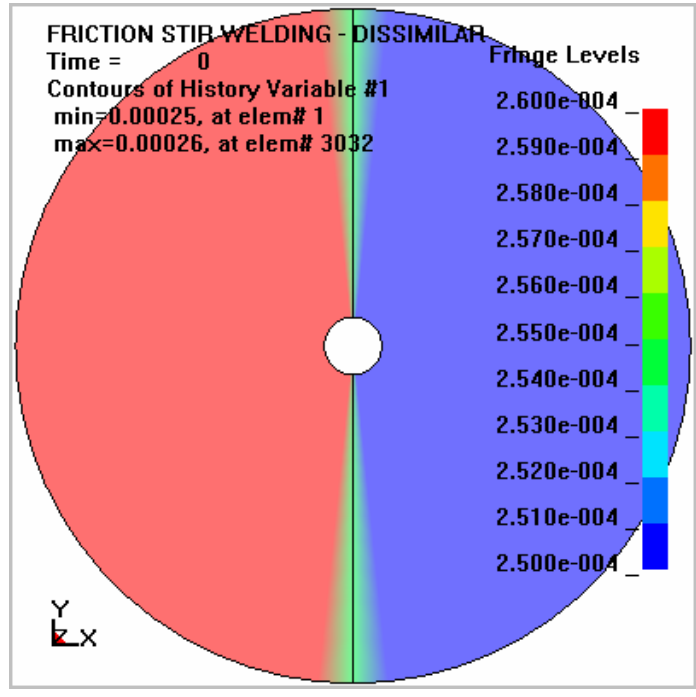

(a) $t=0 \mathrm{~s}$

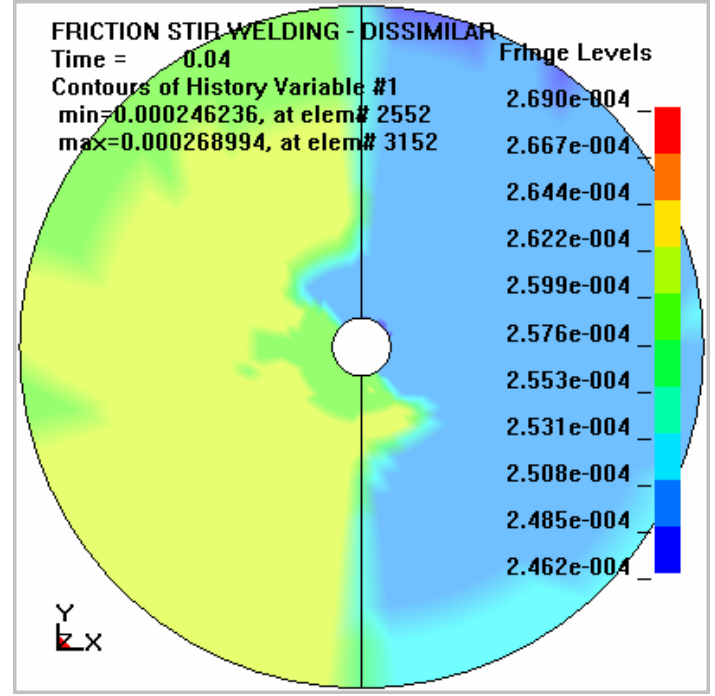

(b) $t=0.04 \mathrm{~s}$ 


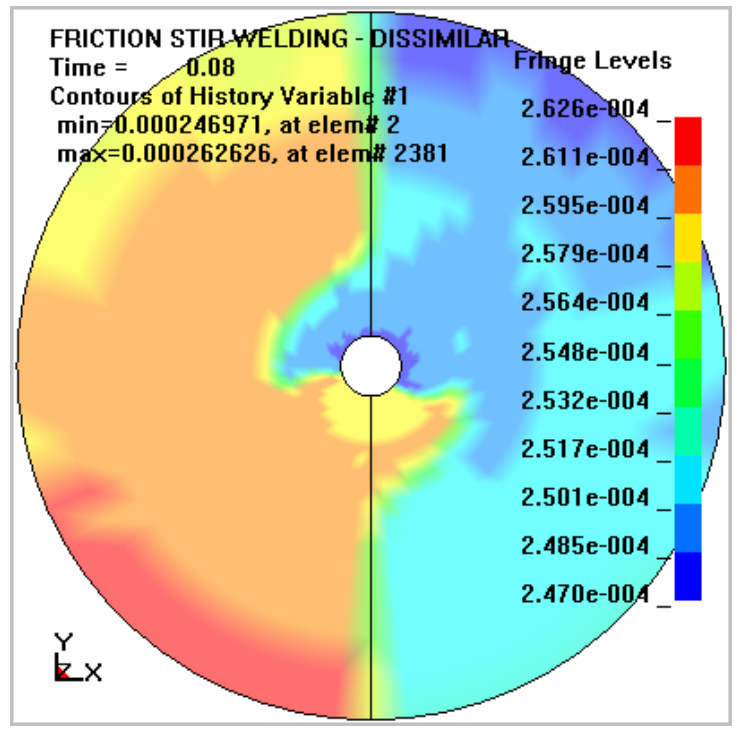

(c) $t=0.08 \mathrm{~s}$

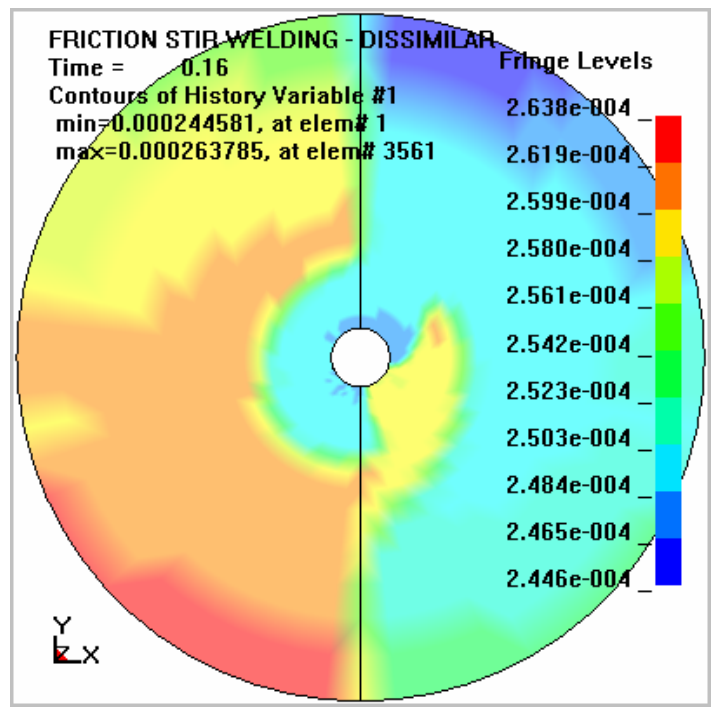

(e) $t=0.16 \mathrm{~s}$

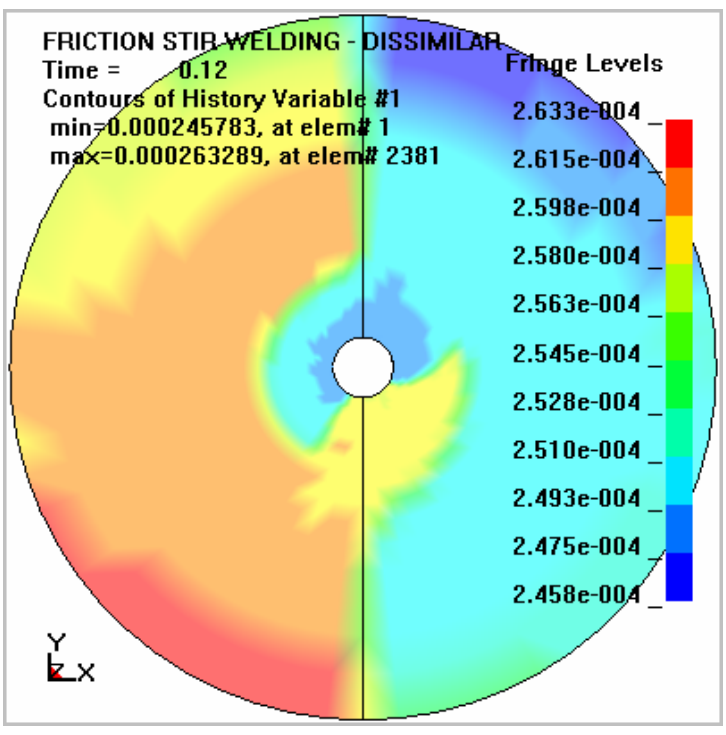

(d) $t=0.12 \mathrm{~s}$

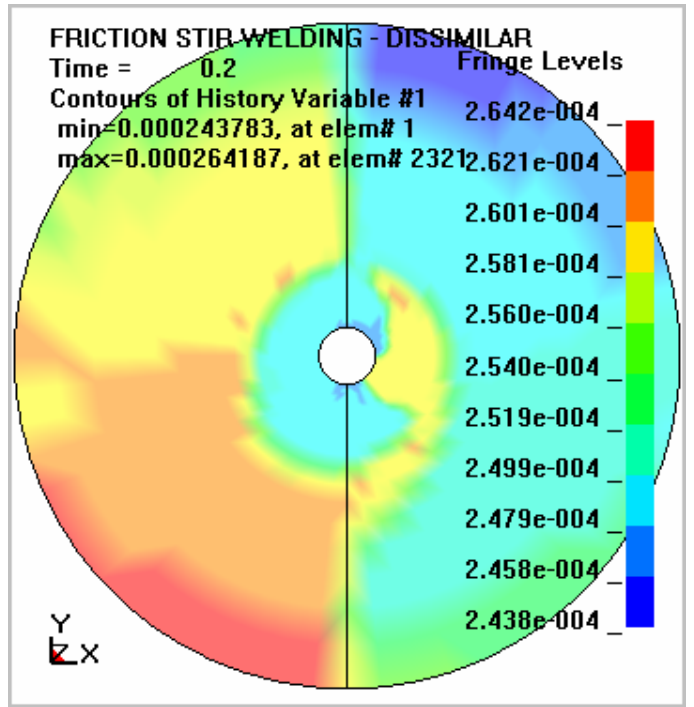

(f) $t=0.2 \mathrm{~s}$

Figure 5-22 Mass Density Contours for the Workpieces at Different Time Instances 


\subsubsection{Case Four - Friction Effect on the Material Flow during FSW 5.2.4.1 The Basic theory of Frictional Contact}

In FSW, the workpieces being joined are subjected to relative motion and pressure so that friction is developed at the region around the welding tool. The basic theory of frictional contact in the FSW process was described in the dissertation of Thorn [59].

Friction is a force that opposes motion in a particular direction. Friction occurs when the surface of one material slides across the surface of another material and some other force is pressing the surfaces together. Since the surfaces in contact with each other are rarely smooth: small projections called asperities are present on the contact surface, reducing the contact area. These asperities, in becoming trapped against each other, provide the resistance to movement which is called friction. If a transverse force is now applied to one of these contact surfaces, then for small values of this force, no movement is observed: this resisting force is called static friction. Above a critical value, however, the asperities fail under the shear stresses present, either by fracture or plastic deformation allowing the surfaces to move. However, the asperities still provide some resistance to movement as they interact, which is known as sliding friction, In sliding friction, the frictional force is normally taken to be proportional to the normal force, although this is only an empiricism due to the complex nature of the contact between the surfaces.

The work done in sliding friction, moving the asperities over and through each other generates heat at the interface. This is concentrated on the small true contact area and the local temperature increases, which if the heat generated is high enough then it can cause significant material softening. As the sliding speed increases, the interface 
temperature rises, but the softening of the material eventually limits this rise by reducing the work done by sliding and moderating the heat generation, The interplay between the temperature, the material hardness and the work done by friction is therefore a selfregulating feature of such frictional contacts.

Increasing the normal force while maintaining the sliding between the two materials causes the asperities to wear and flatten. This in turn increases the true contact area between the surfaces and the amount of heat generation increases. If sliding occurs repeatedly across the same region of surface, the true contact area increases significantly, which, in the limit can completely flatten the asperities and true contact occurs across the whole surface. The temperature now is very high, close to the melting point, and interface region is now extremely soft. This limiting case is called sticking friction and the hightemperature region at the interface is fully plasticized: its behavior in this area is analogous to material undergoing an extrusion process. The mechanism of heat generation is now shearing of this plasticized layer, and is still dependent on the strength of the material. As the strength of the material reduces almost to zero at the melting temperature, this temperature provides an upper limit which is the bulk temperature present in the plasticized region.

\subsubsection{Friction Effect on Material Flow}

In the FSW process, the interface between the workpieces and welding tool experiences frictional contact. Based on the Coulomb friction law, the friction stress is equal to the product of the frictional coefficient, $\mu$, and the contact pressure. In this case, 
the material flow behaviors during FSW are modeled under the different frictional coefficients and forging force displacements.

The problem definition is the same as that in Fig.5-4. The geometries, all reference values, and the chosen material properties are given in Tables 5-8 and 5-9, respectively. Again, the workpiece material is treated as elastic-perfectly plasticity with kinematic hardening, and the pin and shoulder are assumed rigid. Three models are developed with three kinds of combinations of the frictional coefficient and forging force displacement i.e. $\mu=0.47$, forging force displacement $=0.005$ in; $\mu=0.61$, forging force displacement $=0.005$ in; $\mu=0.47$, forging force displacement $=0.001$ in.

Table 5-8 Geometries and Reference Values

\begin{tabular}{c|c|c|c}
\hline & Workpieces & Pin & Shoulder \\
\hline \hline Diameter (in) & 3.0 & 0.2552 & 0.7656 \\
\hline Thickness (in) & 0.125 & 0.12 & 0.05 \\
\hline Translational Velocity & \multicolumn{3}{|c}{$0.2 \mathrm{in} / \mathrm{sec}$} \\
\hline Rotational Velocity & \multicolumn{3}{|c}{$94.2 \mathrm{rad} / \mathrm{sec}(900 \mathrm{rpm})$} \\
\hline Frictional Coefficient & \multicolumn{3}{|c}{$0.47 / 0.61$} \\
\hline Forging & \multicolumn{3}{|c}{$0.005 \mathrm{in} / 0.001 \mathrm{in}$} \\
"Force"(Displacement) & \multicolumn{2}{|c}{}
\end{tabular}

Table 5-9 Mechanical Properties of Material

\begin{tabular}{c|c|c|c|c}
\hline Material & $\begin{array}{c}\text { Mass Density } \\
\left(\left(\mathrm{b}^{*} \mathrm{~s}^{\wedge} 2\right) / \mathrm{in}^{\wedge} 4\right)\end{array}$ & $\begin{array}{c}\text { Young's } \\
\text { Modulus } E \\
(\mathrm{psi})\end{array}$ & $\begin{array}{c}\text { Poisson } \\
\text { Ratio }\end{array}$ & $\begin{array}{c}\text { Yield Stress } \\
\sigma_{y}(\mathrm{psi})\end{array}$ \\
\hline $\begin{array}{c}\text { Workpieces } \\
(\mathrm{Al} \text { 6061-T6) }\end{array}$ & 0.00025 & $1.0 \mathrm{e} 7$ & 0.33 & 39900 \\
\hline $\begin{array}{c}\text { Pin and shoulder } \\
\text { (Steel) }\end{array}$ & 0.00073 & $3.0 \mathrm{e} 7$ & 0.28 & ---- \\
\hline
\end{tabular}


In Fig.5-23, a comparison of the volume fraction contours between the different frictional coefficients and forging force displacements at $t=0.2 \mathrm{~s}$ is presented. Fig.523(a), (b) and (c) shows the models with $\mu=0.47$, forging force displacement $=0.005$ in; $\mu=0.61$, forging force displacement $=0.005$ in and $\mu=0.47$, forging force displacement $=0.001$ in, respectively.

The differences are observed in the advancing and retreating sides for three models mentioned above. The material flow in the model with $\mu=0.61$ is larger than that in the model with $\mu=0.47$ and the material flow in the model with forging force displacement $=0.005$ in is greater than that in the model with forging force displacement $=0.001 \mathrm{in}$. That is because larger frictional coefficient and forging force displacement yields larger friction which produces larger strains. From the simulation results, it is shown that increasing friction results in the increase of plastic strain and increases the mobility of material in the weld. Friction plays an important role in determining the material flow pattern during FSW.
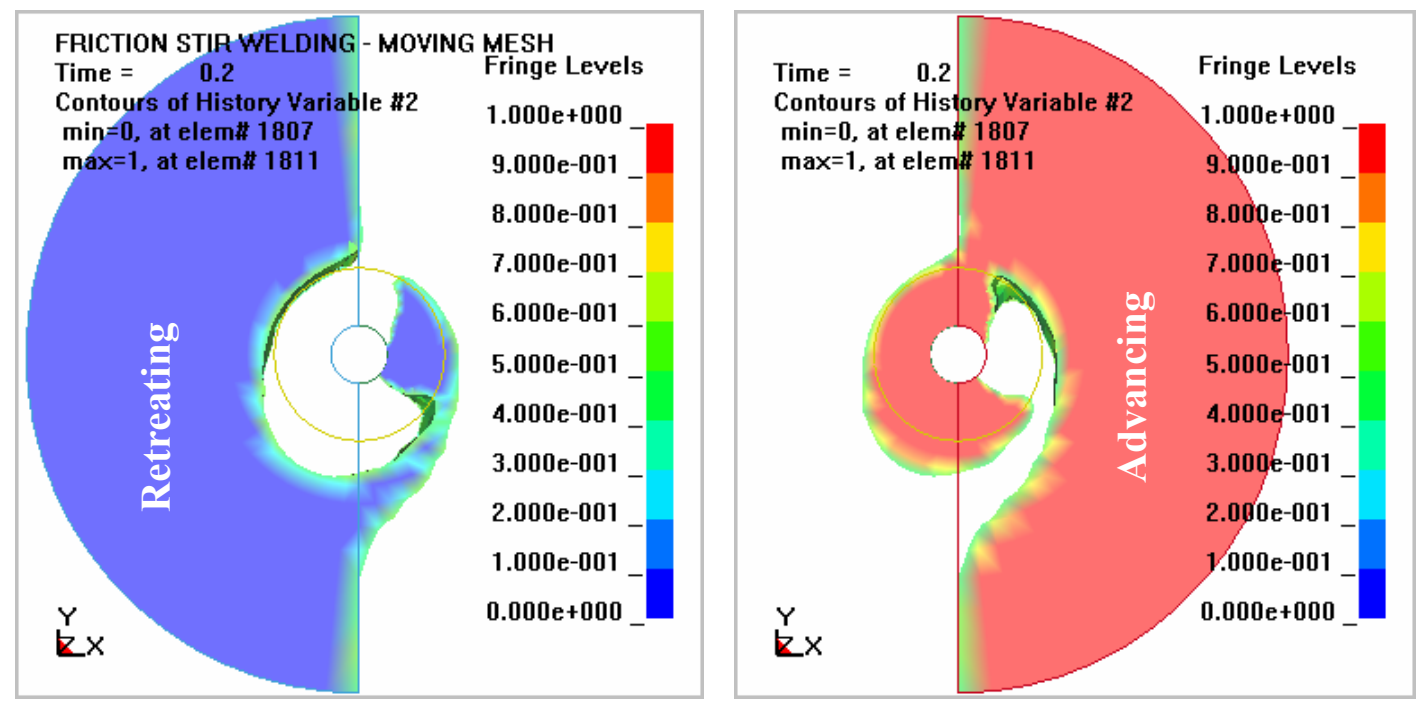

(a) $\mu=0.47$, Forging Force Displacement $=0.005$ in 

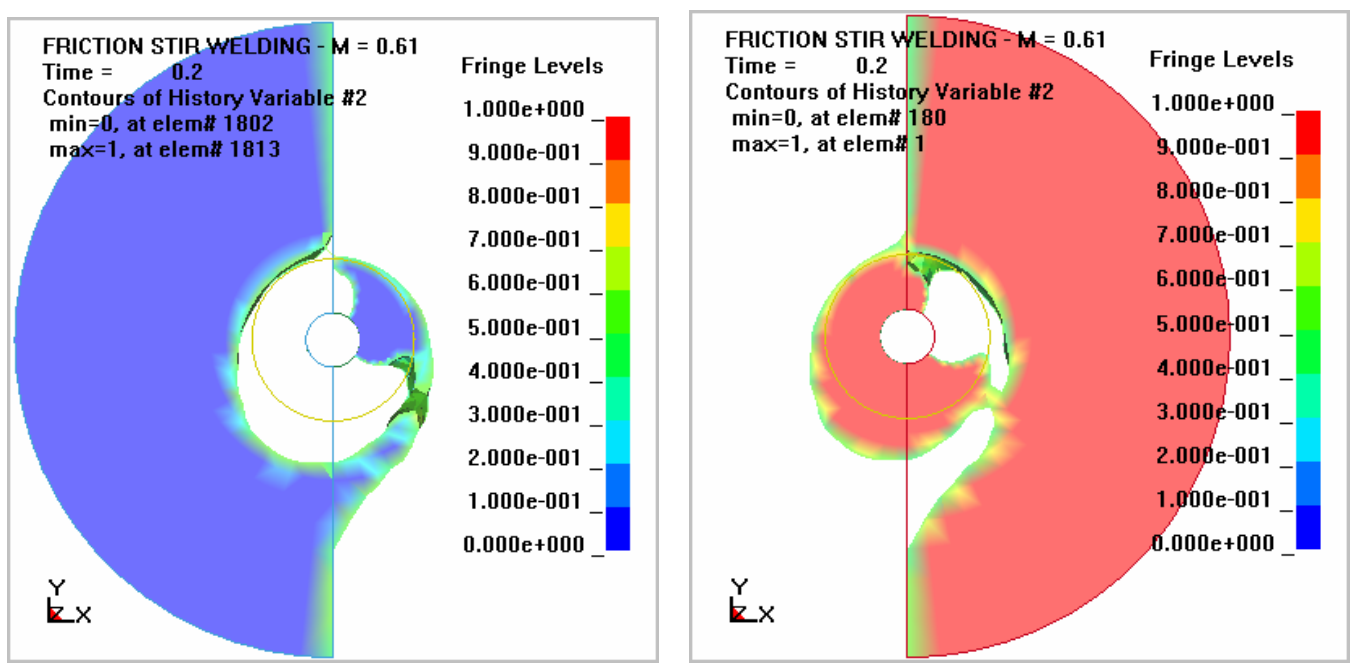

(b) $\mu=0.61$, Forging Force Displacement $=0.005$ in
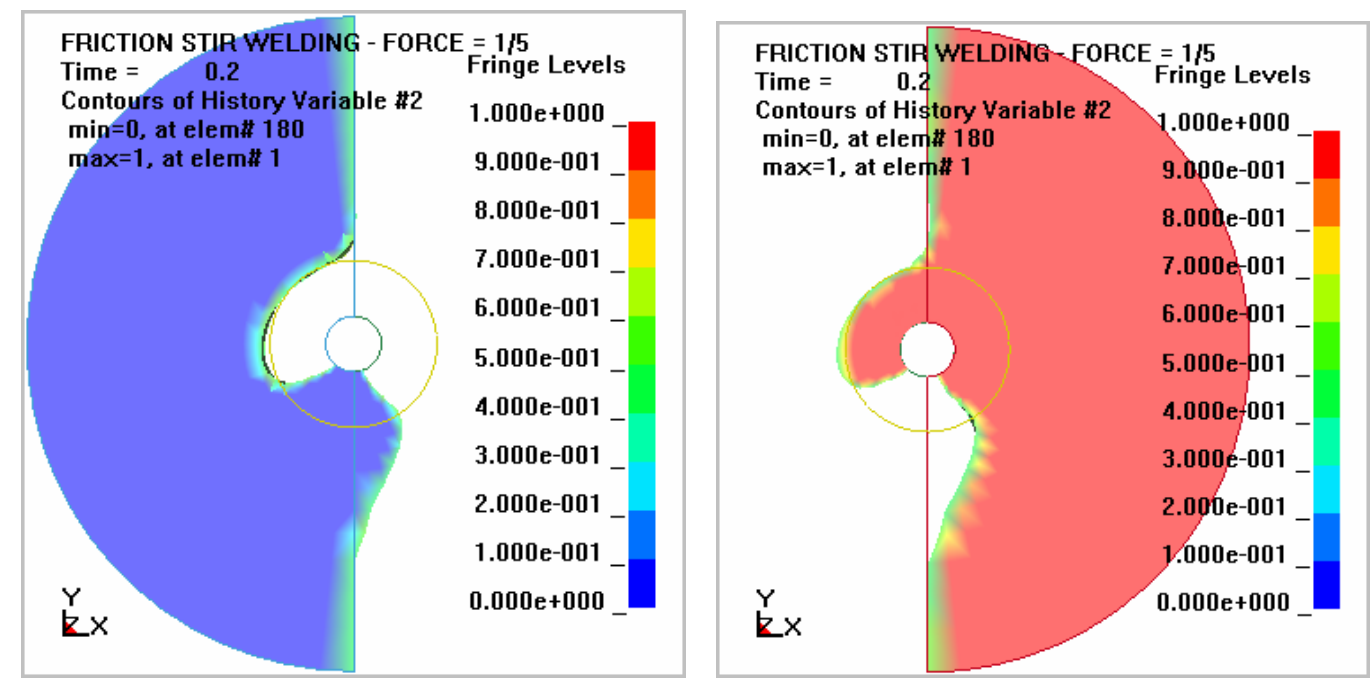

(c) $\mu=0.47$, Forging Force Displacement $=0.001$ in

Figure 5-23 Volume Fraction Contours for the Workpieces with Different Frictional Coefficients and Forging Force Displacement at $t=0.2 \mathrm{~s}$ 
In the simulations shown above, the frictional coefficient is simplified as a constant. Actually, the frictional coefficient is not constant; it is just too complicated to find the exact value. The reason for this is that the frictional coefficient depends on many factors such as asperities, sliding speed, temperature, load and area of contact, and the type of material, etc. Asperities play a major role in determining the frictional coefficient between materials. The frictional coefficient depends on the asperities of the surfaces in contact. The pressure on an asperity is greater than the normal force, that it may deform the contact area plastically. Therefore, frictional force arises from sliding objects breaking and creating bonds created by asperities. Sliding speed also has an important influence on the frictional coefficient. Frictional coefficient is extremely great when the sliding speed is too small and it drops as the speed increases.

Therefore, to increase the friction between the workpieces and welding tool in the FSW process, several ways could be taken: using profiled welding tool instead of cylindrical welding tool for the increase of the asperities on the contact surfaces; decreasing the relative velocity for the increase of the frictional coefficient; increasing the forging force for the increase of the normal force. 


\subsection{Summary}

Four finite element simulations of the material flow in the friction stir butt weld of the same and dissimilar alloys were performed using the moving mesh method based on ALE formulations.

In the first case, the material flow in the friction stir butt weld of the same aluminum alloy was modeled. The volume fraction method and tracer particle technique were used to track the material motion during FSW. By the use of those methods, the material flow around the welding tool was characterized. The simulation predictions of the material flow pattern were found to be consistent with the published experimental results.

In the second case, the material flow patterns in the two material models, namely, power law isotropic plasticity and elastic-perfectly kinematic plasticity were studied. The simulations showed that the material flow patterns were different in both material models. It was also shown that different material behavior could be easily taken into account in the finite element models.

In the third case, the material flow in the friction stir butt weld of the dissimilar aluminum alloys was modeled. The results demonstrated the capability and potential of the finite element models to simulate the FSW of dissimilar aluminum alloys.

The last case investigated friction effect on the material flow pattern in the friction stir weld of the same aluminum alloy. The results showed that friction played an important role in determining the material flow pattern during FSW. 
Through the four case studies above, it is concluded that the moving mesh method based on ALE formulations offers the most efficient modeling approach for FSW. 


\section{CHAPTER 6 DISCUSSION OF RESULTS}

Based on the simulation results, it is shown that the material flow during FSW is not symmetric about the joint line. The material flow patterns on the advancing and retreating sides are different. The stirring of the material occurs at the top surface of the weld where the rotating tool spreads the material with the shoulder around the pin in the rotational direction of the tool. During FSW, some material particles are carried more than once around the rotating pin and some particles move towards the center (around the pin) and downward (depth-wise) of the workpieces. By following several tracer particles used in the model, different motion patterns are produced for the advancing and retreating sides respectively, as are described next.

The motions of the material particles initially at the top surface of the advancing side indicates that most of those particles tend to move toward the center (around the pin) and downwards (depth-wise) in a spiral path. The closer the particles are to the center, the faster they reach the center (pin) and drag down a bit (depth-wise) by the rotating tool as they rotate. Once the particles reach the center, they tend to rotate faster (with the tool) several times, and in some instances they brake away from the center continuing their rotational motions but at a slower rate (no longer stick to the pin). If the particle separates from the pin on the advancing side (for example tracer particle 9 in Fig.6-1), it tends to move back up a bit (depth-wise) and then it turns back towards the center (pin) and again downward (depth-wise) until it reaches the center at a lower depth. These trends are illustrated in the position coordinate history of Fig.6-2 below. 


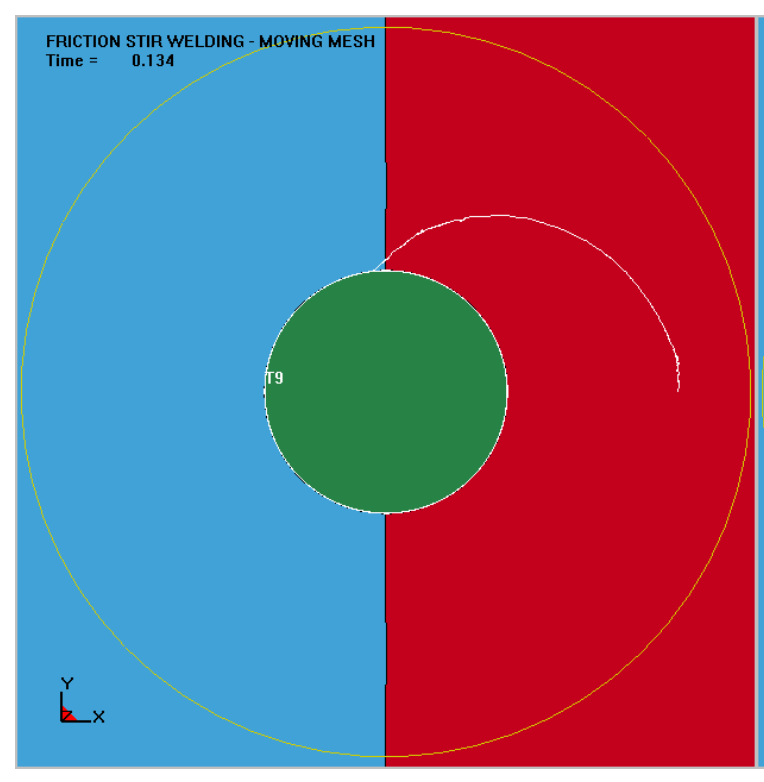

(a)

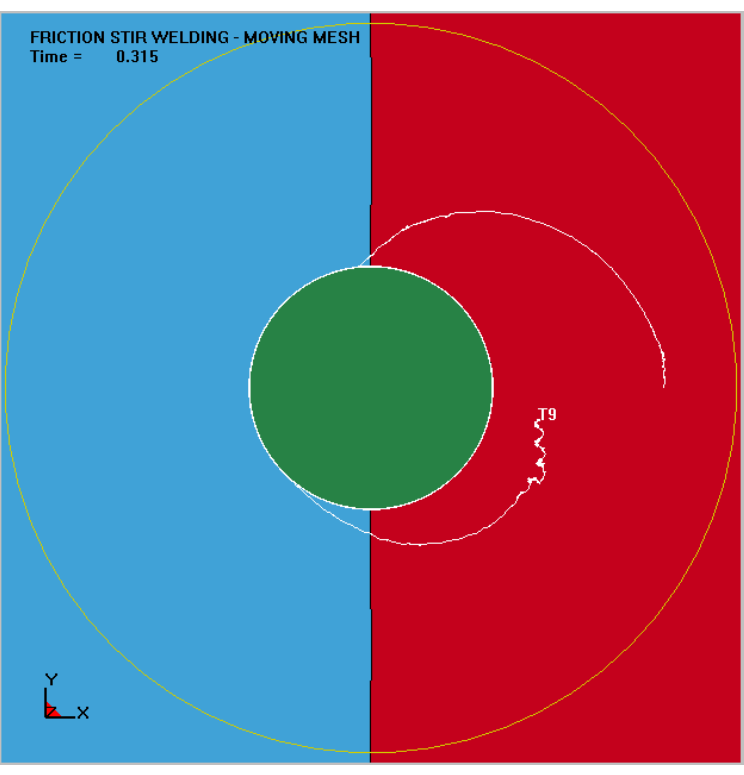

(b)

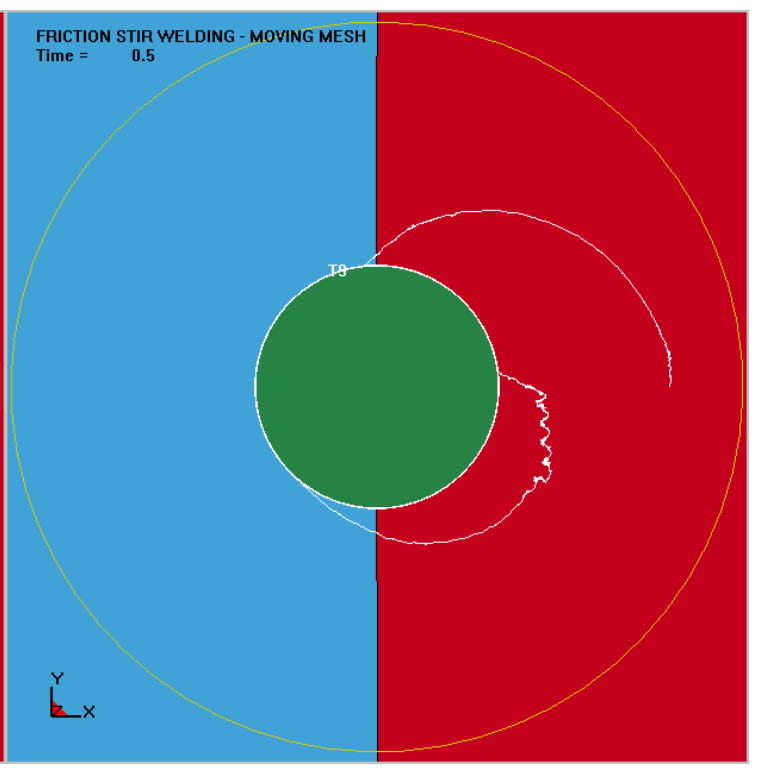

(c)

Figure 6-1 Particle 9 as a Tracing Point, Moving into the Whirlpool then Sticking to the Pin (a); away from the Pin (b); and Spiraling back to the Pin at a Greater Depth (c) 


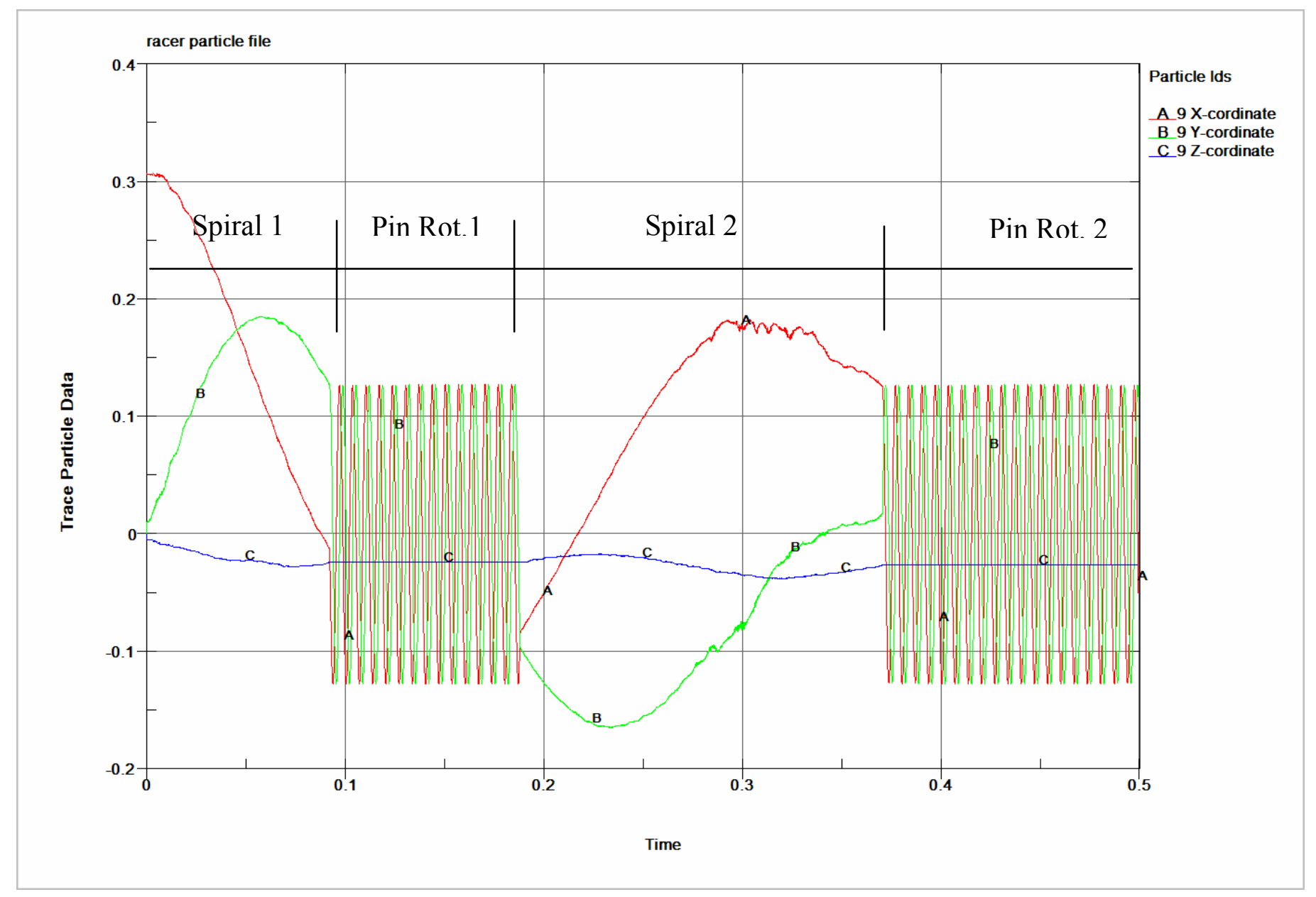

Figure 6-2 Particle 9 as a Tracing Point Coordinates, Moving into the Whirlpool (Spiral1), Sticking to the Pin (Pin Rot. 1), away from the Pin (Spiral 2) and back to the Pin (Pin Rot.2) at a Greater Depth 
The material particles in the retreating side tend to move a bit more circumferentially, with more a tendency toward the outer shoulder circle, and once they reach the outer circle they stay out of the whirlpool. The particles that are closer to the outer circle tend to "fall out" of the whirlpool faster and those particles closer to the center tend to reach the advancing side, falling in the pattern described above. These trends are illustrated in Fig.6-3 and Fig.6-4 below. 


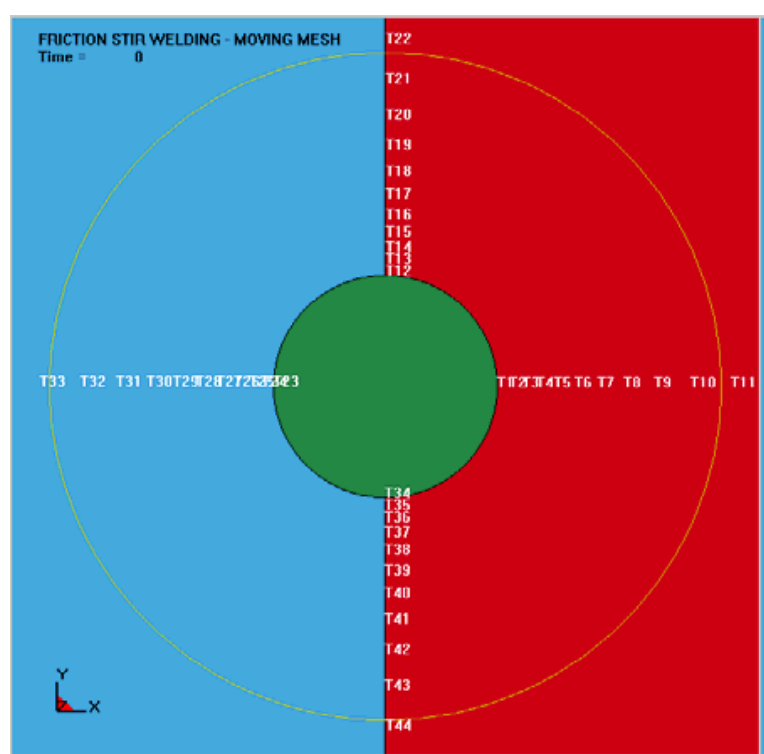

(a) Initial Position

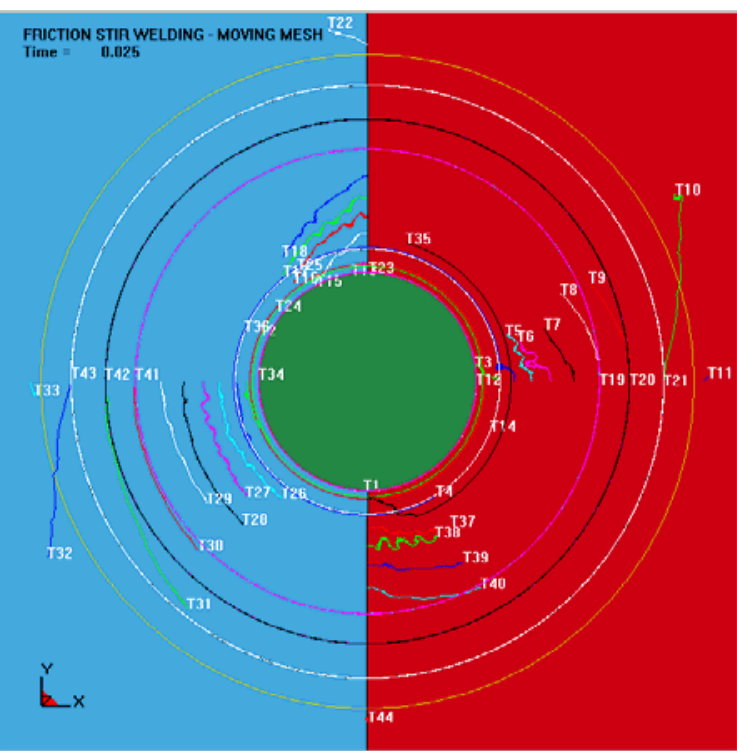

(b) Initial Motion

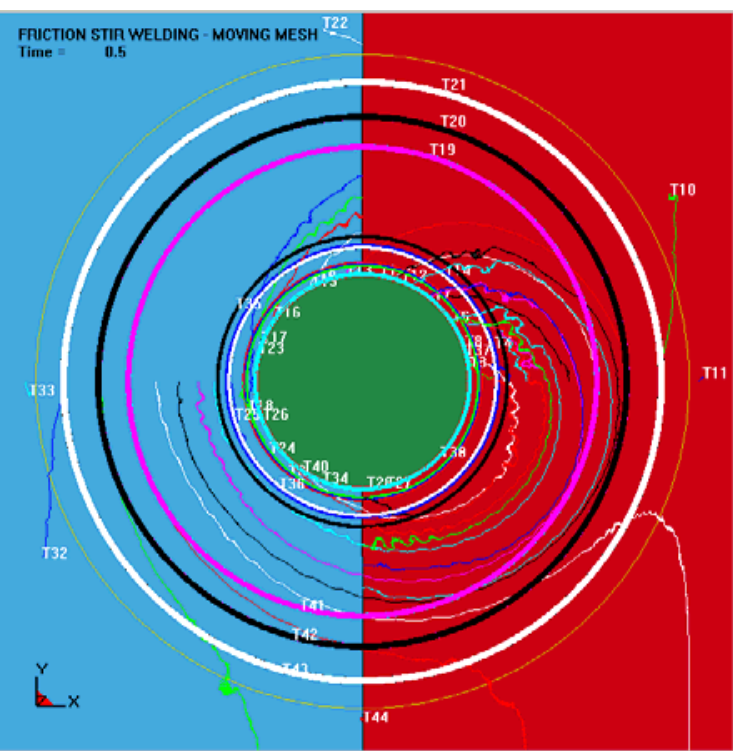

(c) Final Position and Path

Figure 6-3 Sequence of Trace Particle Motions at Beginning, Middle and End of the Simulation 


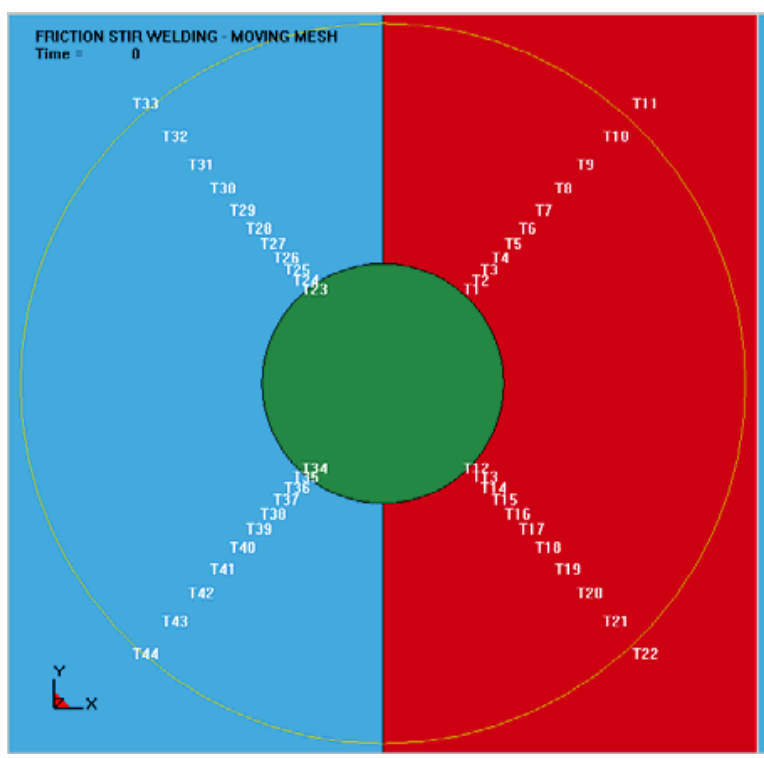

(a) Initial Position

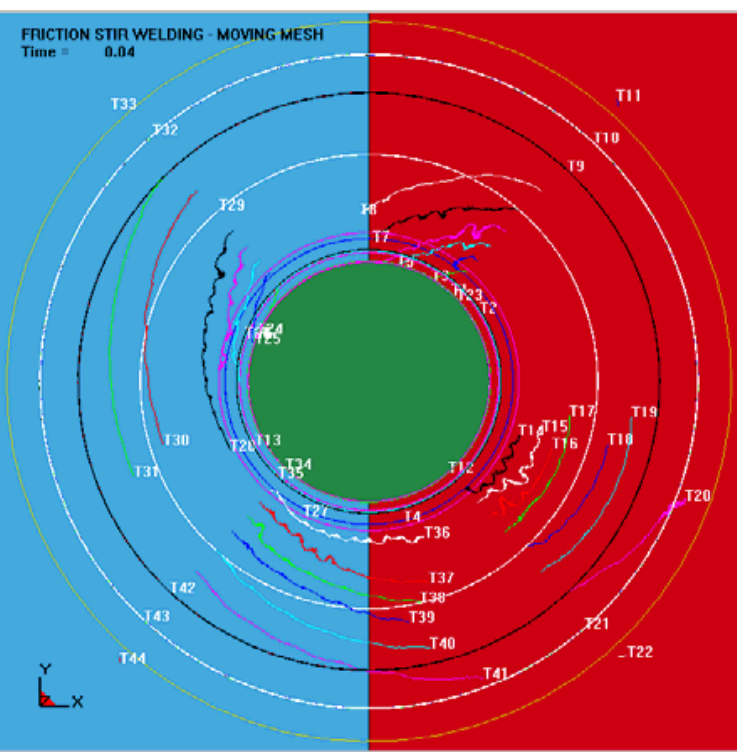

(b) Initial Motion

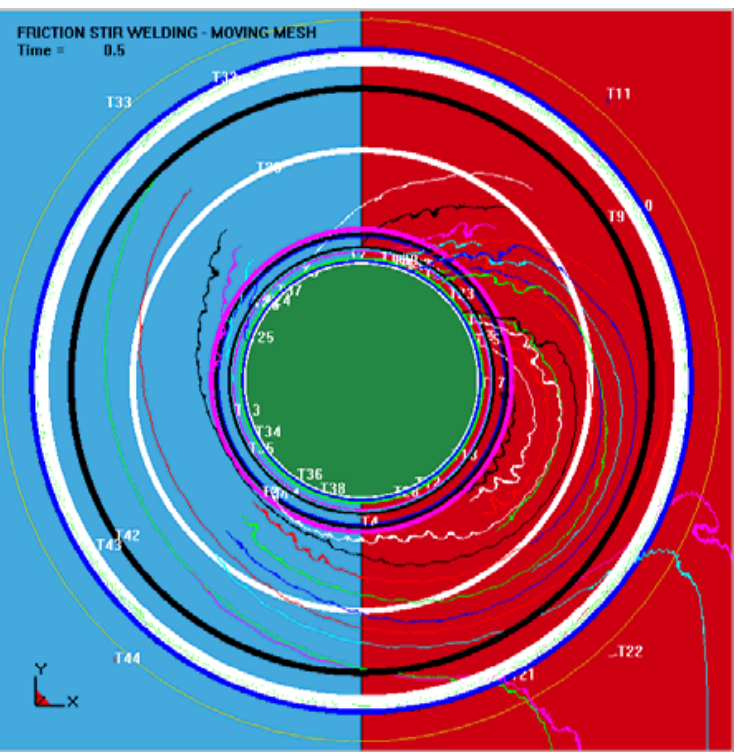

(c) Final Position and Path

Figure 6-4 Sequence of Trace Particle Motions at Beginning, Middle and End of the Simulation 
Overall, there seems to be an intermediate circle (between the pin and shoulder) under which the particles undergo greater motion excursions than throughout the rest of the whirlpool volume. In Fig.6-1 above, particle 9 located at 70\% radial distance between the pin and shoulder radius on the advancing side undergoes the largest excursion of all particles initially located at a $0 \mathrm{deg}$. line (radial line perpendicular to the weld line). These motion trends are consistent with the reported experimental evidence. The closest example is the experimental work conducted by Seidel et al. [52-53], in which a transverse marker band on the top surface of the weld is plotted after the welding tool passes through. Some particles of the advancing side clearly undergo greater motion than others, and those particles are initially at a radius between the pin and shoulder radius, as can be seen in Fig.6-5. This phenomenon can be further observed in the vertical motions of the markers throughout the weld height shown in Fig.6-6. The dark blue marker on the advancing side at the top of the weld has the larger motion between the pin and shoulder radius after welding. Additionally, in Fig.6-5, some particles on the retreating side tend to move more in a circumferential manner and stay behind without reaching the center of the pin. These observations are consistent with the motions described in this simulation. 


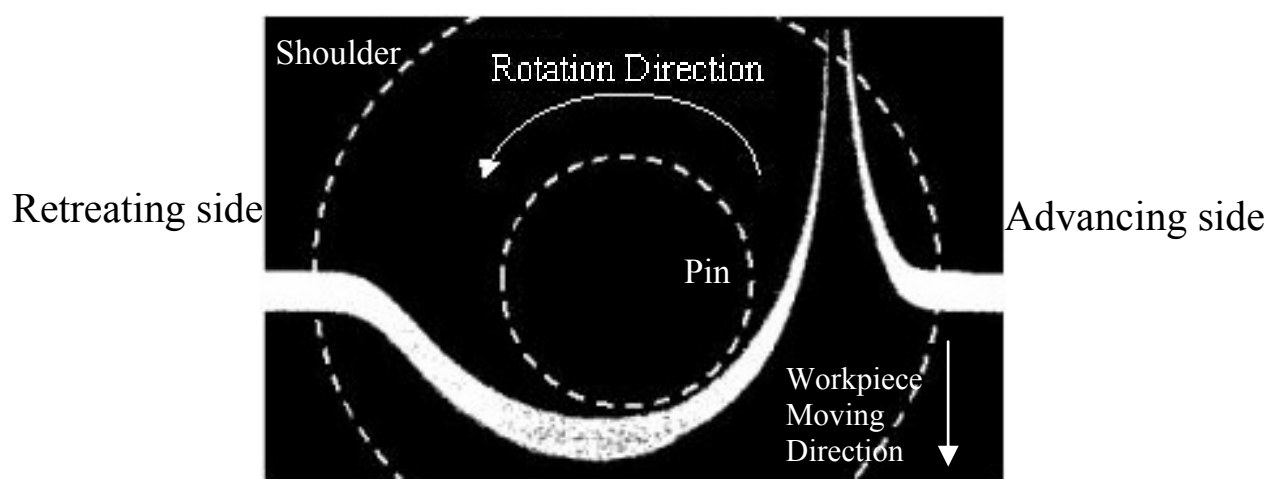

Figure 6-5 Experimental Tracing of Material Motion on the Top of the Workpieces by Seidel et al. [53]

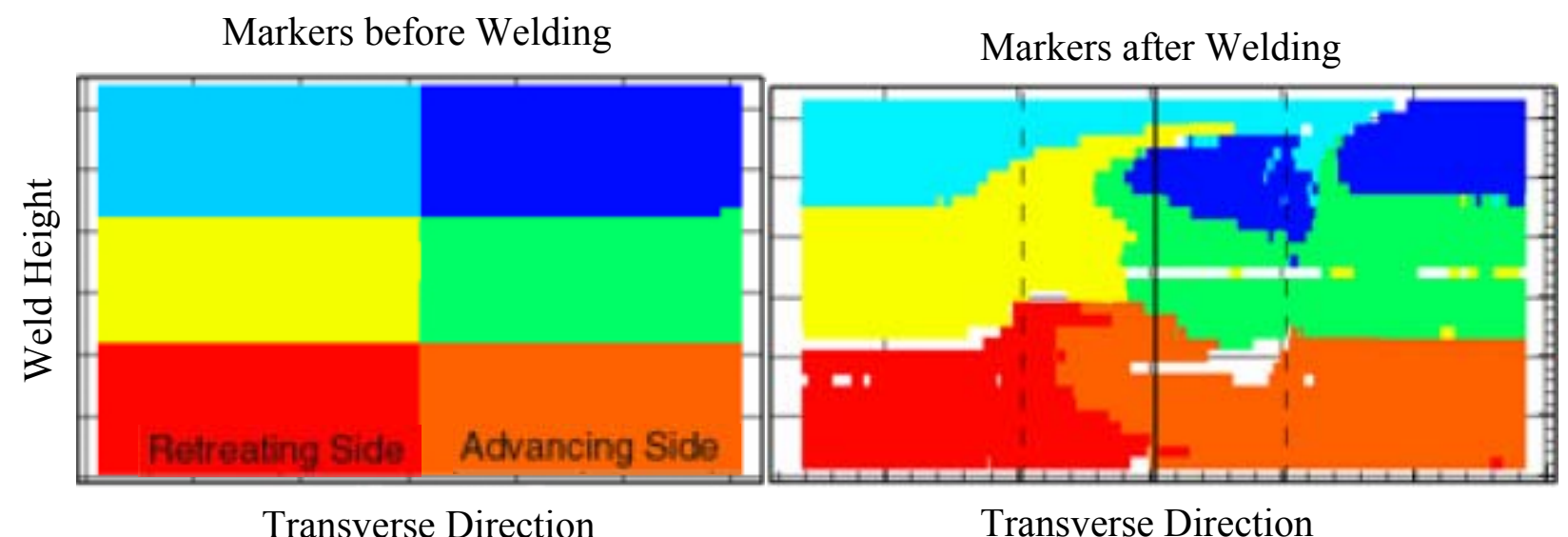

Figure 6-6 Experimental Tracing of Material Motion inside the Workpiece Material (Depth-wise) by Seidel et al. [53] 
A qualitative comparison of the material flow patterns between the finite element predictions with some published experimental data has been made. The simulated motion patterns described above are consistent with the results from the experimental observations in which a marker band on the top surface is used as a "tracer". The tracer band produces a motion pattern for the particles along a transverse line (perpendicular to the welding path). The "deformed" shape of the band records the material motions and two patterns can be identified for the advancing and retreating side respectively. The advancing side shows an intermediate radius in which the particles seem to undergo the greatest displacement (consistent with the numerical simulation). The band seems to thinout as it moves in the tangential direction and then disappear from the surface indicating that those particles actually move into the surface of the workpieces (also consistent with the numerical simulation). Once the particles are under the surface they can no longer be viewed. On the retreating side, the particles seem to perform a circumferential motion and end up spread behind the tool path (also consistent with the numerical simulation). In addition, the observed vertical (depth-wise) motion patterns in the experimental test show that the tracing markers moves downward under the action of the tool forging force first, and then they are forced upward by the backing plate. This vertical flow pattern is also consistent with the simulated results.

It should be stated that in the numerical simulation, some particles close to the outer circle (shoulder) of the tool perform excursions that are inaccurate and not supported by the experimental results, but that may be attributed to a rather large elements in the neighborhood of the shoulder circle. This is just a limitation having to do more with the mesh refinement than the approach and also related to limitations on 
computer processing capacity (as the simulation was conducted on a very basic desktop computer).

The case studies also show that the different material models yield somewhat different flow patterns and different material properties can be easily incorporated into the finite element models developed. It is also shown that friction has an important effect on the material flow pattern in the FSW process. However, the frictional coefficient used is only applied between the rigid tool and workpieces. This uniformly frictional coefficient is at best an oversimplification since the true coefficient is in all likelihood a distributed varying parameter over the contacting surface between the tool and workpiece. In addition, the internal frictional coefficient would play a very important role in the material flow pattern, but was not included in the models in its more realistic form as a distributed friction tensor due to the lack of experimental data to support it. But experimental data on the distributed properties of the internal friction in the material are beyond the scope of this dissertation. 


\section{CHAPTER 7 CONCLUSIONS}

\subsection{Summary}

This dissertation focuses on the finite element simulation of the material flow during Friction Stir Welding (FSW) process and the following aims have been pursued:

1. To develop a mesh motion scheme for simulating the large deformations of the workpieces in the FSW process as an alternate process to adaptive meshing schemes.

2. To assess the material flow behavior of the rigid-elastoplastic problem of FSW using the mesh moving approach in order to establish motion pattern characteristics and overall approach effectiveness.

The most challenging issue in modeling FSW is the problem of the finite element mesh motion as the workpiece material flows around the welding tool. The Lagrangian computations of the FSW process showed that the mesh deformed severely at the locations where the high deformations of material appeared. The approximation accuracy of the elements then deteriorated and rapidly became unstable, and consequently, the calculations were aborted. The difficulty associated with the Lagrangian approach was resolved by the use of Arbitrary Lagrangian-Eulerian (ALE) formulations. In the ALE approach, two sets of coordinate systems are defined: one attached to a material point and moving with the material as it deforms. The other coordinate system is for a corresponding computational point, which moves independently according to a userdefined mesh motion. The selected mesh motion is parallel to the translational motion of the tool. Therefore, the ALE mesh does not get tangled under intense shear. ALE 
formulations were presented in detail in chapter 3. The operator split technique was used to solve the governing equations. With this technique, several Lagrangian steps are followed by an advection step. In the advection step, the distorted mesh at the end of a Lagrangian step is moved back to its original shape, while the strain/displacement solution is remapped to the updated mesh.

An important consideration in applying the ALE approach is the advection method, which determines the motion of the nodes of the finite element mesh in every step of the analysis such that the resulting mesh retains an optimal shape and condition. Specifically in the moving mesh approach, the mesh of the workpieces remains unchanged while the tool moves longitudinally on the workpieces, in such a way that the need for adaptive meshing to fill the hole (in the back) and to open the hole (in the front) along the weld line is completely unnecessary. This feature makes the moving mesh concept an effective and computationally efficient method for the simulation of the FSW process.

As a first example case, the material flow in the friction stir butt weld (using the same aluminum alloy for both workpieces) was modeled to show the performance of the moving mesh method. Then this method was applied to an investigation into the effects of the different material models on the material flow. The material flow patterns in the elastic-perfectly plastic with kinematic hardening model and the power law isotropic plastic model were compared. Furthermore, the material flow in the friction stir butt weld of the dissimilar aluminum alloys was investigated using the same method. Finally, the effect of friction on the material flow was studied using the same method again. 


\subsection{Contribution to the State of the Art in FSW Simulation}

FSW involves a very large plastic deformation process similar to a fluid in a whirlpool but a material does not behave like a fluid but more as a plastic material under an extrusion whirlpool. The process while successfully implemented in industry has proved elusive for comprehensive experimental and numerical descriptions due to the very nature of the process. Yet, from the perspective of numerical simulation, very few models and procedures have been successfully applied to describing the mechanics of this process. In this dissertation, an approach has been developed, implemented and applied, under some simplified assumptions, which allow a visualization of material motion in the whirlpool of the FSW process. While the various numerical methods have existed for a while, the contribution (albeit modest) in this dissertation resides in the effective utilization of the ALE into the concept of "moving mesh", which allows the material motion to be traced while the elastoplastic state of the material is determined throughout the volume of the workpieces, and specifically through out the whirlpool volume. In order to enable further applications and study of the FSW process, the "moving mesh" approach has been summarized in the form of a flowchart, which describes the procedure and is shown in Fig.7-1 below. This chart illustrates the overall steps followed and later on implemented for execution with an explicit finite element code (namely LS-DYNA), but could be followed with other explicit finite element codes.

This approach can be used as a tool to produce material motion trends in FSW processes for welds with same material for the workpieces, or different materials properties and for various process geometries and parameters. Furthermore, other 
important physical aspects of the process can be addressed through simulation such as internal friction characterization needed to gain a better understanding of the process.

\subsection{Conclusions}

Based on the material flow trends observed, it can be concluded that:

1. The material motion characteristics for the advancing and retreating sides of a weld have been respectively made. The characteristics of the motion were illustrated by means of tracing particles attached to the material. While limited experimental evidence is available the trends observed are reasonable consistent with some experimental results, which partially show the motions of some markers inserted into a weld.

2. On the advancing side (considering a ccw rotation and a forward longitudinal motion of the tool), most particles above the 0 deg. line tend to spiral toward the pin and into the thickness of the plates. Particles below the 0 de. Line, tend to rotate in a circumferential manner and if they are closer to the shoulder radius they tend to "fall out" of the whirlpool and stay behind the weld. Otherwise they continue to spiral toward the pin.

3. On the retreating side particles tend to move more on a circumferential way, following the tool rotation (ccw) and they also tend to move towards the shoulder circumference prior to "falling-off" the whirlpool.

4. The material on the surface is dragged circumferentially by friction by the tool and there seems to be a radial position at approximately $70 \%$ distance between 
from the pin to the shoulder, and slightly above the 0 deg. line, where the particles experience the greatest excursions.

5. Along the depth of the weld, the particles are pushed downward by the welding tool and then go upward due to the reaction of the backing plate.

6. From the simulations performed, it is also evident that the frictional coefficient between the tool and the workpieces has an impact on the overall motion of the particles in the whirlpool of the weld, but the use of a constant frictional coefficient is at best a simplification which is beyond the scope of this dissertation, but identified as an important process response parameter.

7. The material elastoplastic behavior has a significant impact on the overall behavior of the material flow. Only two models were used in this dissertation and the patterns described above are limited to the assumptions of the two material models used; (a) the elastic-perfectly plastic material with kinematic hardening and (b) the power law isotropic plastic material. A more realistic approach would be the use of temperature dependant elastoplastic material properties.

It is finally concluded that due to the characteristics of the FSW process, the moving mesh method based on ALE formulations offers an efficient modeling approach. Case studies have demonstrated the capabilities and potential of this method in simulating the FSW process. The approach developed can be used to evaluate materials and tool designs and study the welding parameters for optimizing the FSW process of different alloys. 


\subsection{Future Directions}

Some promising FSW simulations have been performed and the moving mesh method based on ALE formulations seems to be numerically robust. However, the simulations presented in this work involve a constitutive model which is independent of thermal and strain rate effects. Future direction should include more realistic material models. Secondly, the model should drop the simplification of assuming that the pin is a cylinder. Future models should include the thread of the pin. Thirdly, as we have known, friction has a great effect on the martial flow in FSW. In the current models, only external friction is considered and the frictional coefficient is simplified as a constant. Actually, due to the shear deformation of material, the relative movement exists between two elements and then an internal friction produces at the moving layers relative to each other. In addition, the internal frictional coefficient is different at every point within the material body and relates to the magnitude and the direction of material deformation. In the future models, the internal friction of the material body itself should be taken into account and the internal frictional coefficient at every point may be treated as a scalar or a tensor if it is related to the material strain tensor. 
- Geometry: define nodes and elements

- Materials: define 4 parts for the two workpieces, the pin and shoulder and specify element formulation, constitutive data, hourglass control for one point integration

- Constraints: apply constrains among the workpieces, the pin and shoulder.

- Boundary Conditions \& Loading: apply restraints to the workpieces and welding tool, prescribe load of the tool on the workpieces, and define load curves

- Control: reset default global parameters for ALE computation, energy dissipation option, hourglass type, time step size and termination time

- Database: control the output of ACCII time history and binary plot files

Lagrangian step: determine the Lagrangian solution

Replace $n$ by $n+1$ and go to step 2
Step 1 Initialization. Set $n=0$, input initial conditions

Step 2 Time stepping loop, $t \in\left[0, t_{\max }\right]$

Step 3 Compute the mesh velocity

Step 4 Compute the mesh displacement and spatial coordinates

Step 5 Compute the stress and mass density

Mesh smoothing: determine the mesh velocity by a smoothing algorithm to obtain the convective velocity

Step 6 Compute the final position of the nodes due to a mesh smoothing procedure.

Step 7 Compute the mesh velocity Step 8 Compute the convective velocity

Solution mapping: determine the final solutions

Step 9 Compute the stress, mass density from their Lagrangian solutions to their final ones.

Step 10 Compute the velocity from its Lagrangian solution to its final solution

Lagrangian step

Step 11 Compute the internal force vector

Step 12 Compute nodal acceleration

No

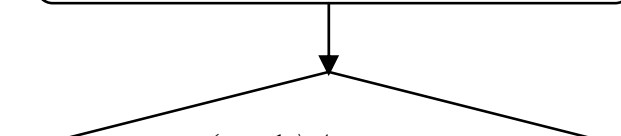

$$
\text { If }(n+1) \Delta t>t_{\max }
$$

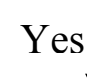

Stop

Figure 7-1 Procedure Flowchart of the ALE Moving Mesh Approach for FSW Simulation 


\section{REFERENCE}

[1] Askari A., S. Silling, B. London, M. Mahoney, "Modeling and Analysis of Friction Stir Welding Process", Friction Stir Welding and Processing, TMS 2001, Indianapolis, Indiana, USA, November 4-8, 2001, pp. 43-54

[2] Belytschko T., W.K. Liu, B. Moran, Nonlinear Finite Elements for Continua and Structures, 2000

[3] Bendzsak G.J., T.H. North, C.B. Smith, “An Experimentally Validated 3D Model for Friction Stir Welding", $2^{\text {nd }}$ International Symposium on FSW, Gothenburg, Sweden, 2000

[4] Benson D.J., "An Efficient, Accurate, Simple ALE Method for Nonlinear Finite Element Programs", Computer Methods in Applied Mechanics and Engineering, 1989, 72(3), pp. 305-350

[5] Chao Y.J., X. Qi, W. Tang, "Heat Transfer in Friction Stir Welding - Experimental and Numerical Studies", Journal of Manufacturing Science and Engineering, 2003, 125, pp.138-145

[6] Chao Y.J. X. Qi, "Thermal and Thermo-Mechanical Modeling of Friction Stir Welding of Aluminum Alloy 6061-T6", Journal of Materials Processing \& Manufacturing Science, 1998, 7, pp.215-233

[7] Chen C.M., R. Kovacevic, "Finite Element Modeling of Friction Stir Welding Thermal and Thermomechanical Analysis", International Journal of Machine Tools \& Manufacture, 2003, 43, pp.1319-1326 
[8] Colegrove P., H. Shercliff, "2-Dimensional CFD Modeling of Flow Round Profiled FSW Tooling", Friction Stir Welding and Processing I, TMS 2003, San Diego, California, USA, March 2-6 2003, pp.13-22

[9] Colligan K., "Material Flow Behavior during Friction Stir Welding of Aluminum", Welding Journal Research Supplement, 1999, pp.229s-237s

[10] Deng X., S. Xu, “ Two Dimensional Finite Element Simulation of Material Flow in the Friction Stir Welding Process", Journal of Manufacturing Processes, 2004, 6(2), pp.125-133

[11] Dickerson T., H.R. Shercliff, H. Schmidt, "A Weld Marker Technique for Flow Visualization in Friction Stir Welding", 4th International Symposium on Friction Stir Welding, Park City, Utah, U.S.A, May 14-16 2003

[12] Dickerson T., Q-Y. Shi, H.R. Shercliff, "Heat Flow into Friction Stir Welding Tools", $4^{\text {th }}$ International Symposium on Friction Stir Welding, Park City, Utah, 14-16 May 2003

[13] Donea J., “Arbitrary Lagrangian-Eulerian Finite Element Methods”, Computational Methods for Transient analysis, Edited by T. Belytschko et al. , 1983, pp.473-516

[14] Donea J. A. Huerta, J.-Ph. Ponthot, A. Rodríguez-Ferran, “Arbitrary LagrangianEulerian Methods", Encyclopedia of Computational Mechanics, 2004, Edited by Erwin Stein et al, 1

[15] Dong P., F. Lu, J.K. Hong, Z. Cao, “Coupled Thermomechanical Analysis of Friction Stir Welding Process Using Simplified models", Science and Technology of Welding and Joining, 2001, 6(5), pp. 281-287 
[16] Fernandez G.J., L.E. Murr, "Characterization of Tool Wear and Weld Optimization in the Friction-stir Welding of Cast Aluminum 359+20\% SiC Metal-matrix Composite", Materials Characterization, 2004, 52 (1), pp. 65-75

[17] Flanagan D.P., T. Belytchko, “A Uniform Strain Hexahedron and Quadrilateral with Orthogonal Hourglass Control", International Journal for Numerical Methods in Engineering, 1981,17, pp. 679-706

[18] Gadala M.S., J. Wang, "ALE formulation and its application in solid mechanics", Computer Methods in Applied Mechanics and Engineering, 1998, 167, pp.33-55

[19] Gadala M.S., M.R. Movahhedy, J. Wang, "On the Mesh Motion for ALE Modeling of Metal Forming Processes", Finite Elements in Analysis and Design, 2002, 38(5), pp. $435-459$

[20] Ghosh S., "Arbitrary Lagrangian-Eulerian Finite Element Analysis of Large Deformation in Contacting Bodies", International Journal for Numerical Methods in Engineering , 1992, 33(9), pp.1891-1925

[21] Ghosh S., N. Kikuchi, “An Arbitrary Lagrangian-Eulerian Finite Element Method for Large Deformation Analysis of Elastic-viscoplastic Solids", Computer Methods in Applied Mechanics and Engineering, 1991, 86(2), pp.127-188

[22] Goetz R.L., K.V. Jata, "Modeling Friction Stir Welding of Titanium and Aluminum Alloys", Friction Stir Welding and Processing, TMS 2001, Indianapolis, Indiana, USA, November 4-8, 2001, pp. 35-42

[23] Gould J.E., Z. Feng, "Heat Flow Model for Friction Stir Welding of Aluminum Alloys", Journal of Material Processing and Manufacturing Science, 1998, 7, pp.185-194 
[24] Guerra M., J.C. McClure, L.E. Murr, A.C. Nunes, "Metal Flow During Friction Stir Welding", Friction Stir Welding and Processing, TMS 2001, Indianapolis, Indiana, USA, November 4-8, 2001, pp. 25-34

[25] Haber R.B., B.H. Hariandja, "Computational Strategies for Nonlinear and Fracture Mechanics Problems. An Eulerian-Lagrangian Finite Element Approach to Large Deformation Frictional Contact", Computers and Structures, 1985, 20(1-3), pp.193201

[26] Hallquist J.O., LS-DYNA Theoretical Manual, Livermore Software Technology Corporation, 1998

[27] http://www.twi.co.uk

[28] http://www.msm.cam.ac.uk/phase-trans/2003/FSW/aaa.html

[29] http://www.hitachi-rail.com/rail_now/seminor/fsw/fsw2_1.html

[30] A. Huerta, F. Casadei, "New ALE Applications in Nonlinear Fast-Transient Solid Dynamics", Engineering Computations, 1994, 11(4), pp. 317-345

[31] Huétink J., P.T. Vreede, van der Lugt J., "Progress in Mixed Eulerian-Lagrangian Finite Element Simulation of Forming Processes", International Journal for Numerical Methods in Engineering, 1990, 30(8), pp.1441-1457

[32] Johnson R., P, Threadgill, "Friction Stir Welding of Magnesium Alloys", Magnesium Technology, TMS 2003, San Diego, CA, USA, 2-6 Mar. 2003, pp. 147-152

[33] Kimapong K., T. Watanabe, "Friction Stir Welding of Aluminum Alloy to Steel", Welding Journal, 2004, pp. 277S-282S 
[34] Koh H.M., H.S. Lee, R.B. Haber, "Dynamic Crack Propagation Analysis Using Eulerian-Lagrangian Kinematic Descriptions”, Computational Mechanics, 1988, 3, pp.141-155

[35] Li N., T-Y. Pan, R.P. Cooper, D.Q. Houston, Z. Feng, M.L. Santella, "Friction stir Welding of Magnesium AM60 Alloy”, Magnesium Technology, TMS 2004, Charlotte, NC, USA, 14-18 Mar. 2004, pp. 19-23

[36] Lienert T.J., W.L. Stellwag JR, B.B. Grimmett, R.W. Warke, "Friction Stir Welding Studies on Mild Steel”, Welding Journal Research Supplement, 2003, pp.1S-9S

[37] Linder C.,"An Arbitrary Lagrangian-Eulerian Finite Element Formulation for Dynamics and Finite Strain Plasticity Models”, a Master Thesis, 2003

[38] Liu W.K., H. Chang, J.-S. Chen, T. Belytschko, "Arbitrary Lagrangian-Eulerian Petrov-Galerkin Finite Elements for Non-linear Continua", Computer Methods in Applied Mechanics and Engineering, 1988, 68(3), pp. 259-310

[39] London B., M. Mahoney, W. Bingel, M. Calabrese, R.H. Bossi, D. Waldron, "Material Flow in Friction Stir Welding Monitored with Al-SiC and Al-W Composites Markers", Friction Stir Welding and Processing II, TMS 2003, San Diego, California, USA, March 2-6 2003, pp. 3-12

[40] LS-DYNA User's Manual, Livermore Software Technology Corporation, 42003

[41] Movahhedy M., M.S. Gadala, Y. Altintas, "Simulation of Elastic-Plastic Crack Propagation Using Arbitrary Lagrangian-Eulerian Finite Element Method", Proceedings of the $12^{\text {th }}$ ASCE Engineering Mechanics Conference, La Jolla, CA, 1998, pp.110-113 
[42] Movahhedy M., M.S. Gadala, Y. Altintas, "Simulation of the Orthogonal Metal Cutting Process Using an Arbitrary Lagrangian-Eulerian Finite Element Method”, Journal of Materials Processing Technology, 2000, 103, pp. 267-275

[43] Olovsson L., L. Nilsson, K. Simonsson, "An ALE formulation for the Solution of Two-dimensional Metal Cutting Problems”, Computers and Structures, 1999, 72, pp. 497-507

[44] Ouyang J.H., R. Kovacevic, "Material Flow and Microstructure in the Friction Stir Butt Welds of the Same and Dissimilar Aluminum Alloys", Journal of Materials Engineering and Performance, 2002, 11(1), pp. 51-63

[45] Prado R.A., L.E. Murr, A.R. Rivera, D.J. Shindo, K.F. Soto, G.J. Fernandez, J.C. McClure, "Tool Wear and Shape Optimization in the Friction-stir Welding of Aluminum Metal-matrix Composites”, Friction Stir Welding and Processing II, TMS 2003, San Diego, California, USA, 2-6 Mar. 2003, pp.23-32

[46] Prado R.A., L.E. Murr, K.F. Soto, J.C. McClure, "Self-optimization in Tool Wear for Friction-stir Welding of $\mathrm{Al} 6061+20 \% \quad \mathrm{Al}_{2} \mathrm{O}_{3}$ MMC", Materials Science and Engineering: A, 2003, 349(1), pp. 156-165

[47] Reynolds A.P., “Visualization of Material Flow in Autogenous Friction Stir Welds”, Science and Technology of Welding and Joining, 2000, 5(2), pp.120-124

[48] Reynolds A.P., W. Tang, M. Posada, J. Deloach, "Friction Stir Welding of DH36 Steel”, Science and Technology of Welding \& Joining, 2003, 8(6), pp. 455-460

[49] Rodríguez-Ferran A., A. Prez-Foguet, A. Huerta, “Arbitrary Lagrangian-Eulerian (ALE) Formulation for Hyperelastoplasticity", International Journal for Numerical Methods in Engineering, 2002, 53(8), pp. 1831-1851 
[50] Schmidt H., J. Hattel, J. Wert, "An Analytical Model for the Heat Generation in Friction Stir Welding", Modeling and Simulation in Materials Science and Engineering, 2004, 12, pp. 143-157

[51] Schmidt H., J. Hattel, “ A Local Model for the Thermomechanical Conditions in Friction Stir Welding", Modeling and Simulation in Materials Science and Engineering, 2005, 13, pp.77-93

[52] Seidel T.U., A.P. Reynolds, "Visualization of the Material Flow in AA2195 FrictionStir Welds Using a Marker Insert Technique”, Metallurgical and Materials Transactions A, 2001, 32A, pp. 2879-2884

[53] Seidel T.U., A.P. Reynolds, "Material Flow in Friction Stir Welding: Experiment and Fluid Mechanics based Process Model", http: // www. me. sc. edu/ Research/ cmmnde/Friction/ papers/ proc/ mflow.htm

[54] Somasekharan A.C., L.E. Murr, "Microstructures in Friction-stir Welded Dissimilar Magnesium Alloys and Magnesium Alloys to 6061-T6 Aluminum Alloy”, Materials Characterization, 2004, 52(1), pp. 49-64

[55] Song M., R. Kovacevic, "Thermal Modeling of Friction Stir Welding in a Moving Coordinate System and its Validation", International Journal of Machine Tools \& Manufacture, 2003, 43, pp.605-615

[56] Storjohann D., S.S. Babu, S.A. David, Phil Sklad, "Friction Stir Welding of Aluminum Metal Matrix Composites", http: //www. ornl. gov/ webworks/ cppr/ y2001/pres/ 114598.pdf 
[57] Thomas W.M., E.D. Nicholas, J.C. Needham, M.G. Murch, P. Templesmith, and C.J. Daws, "Friction Stir Butt Welding”, GB Patent Application No. 9125978.8, Dec. 1991, U. S. Patent No. 5460317, Oct. 1995

[58] Thomas W.M., P.L. Threadgill, E.D. Nicholas, "Feasibility of Friction Stir Welding Steel”, Science and Technology of Welding and Joining, 1999, 4 (6), pp. 365-372

[59] Thorn G.J., "Mathematical and Computational Modeling of Friction Stir Welding", Ph.D. Dissertation, Department of Applied Mathematics and Theoretical Physics, University of Cambridge, 2004

[60] Ulysse P., "Three-dimensional Modeling of the Friction Stir Welding Process", International Journal of Machine Tools \& Manufacture, 2002, 42, pp.1549-1557

[61] Xu S., X. Deng, A.P. Reynolds, T.U. Seidel, "Finite Element Simulation of Material Flow in Friction Stir Welding", Science and Technology of Welding and Joining, 2001, 6(3), pp.191-193

[62] Ying L., E.A. Trillo, L.E. Murr, "Friction Stir Welding of Aluminum Alloy 2024 to Silver”, Journal of Materials Science Letters, 2000, 19(12), pp.1047-1051 


\section{APPENDIX}

\section{A NOMENCLATURE}

Here is a list of symbols used in the work:

Symbol Definition

Material acceleration

$\hat{\mathbf{a}}$

Mesh acceleration

$A_{\text {cont }} \quad$ Area of the segment contacted by the node in contact

$b_{i} \quad$ Body force per unit volume

$c_{d} \quad$ Exponential decay coefficient

$c_{j} \quad$ Convective velocity

$\mathbf{c}_{I}(t) \quad$ Convective velocity of node $I$

$c_{v d} \quad$ Viscous damping coefficient

$C_{i j k l}^{\sigma} \quad$ Elastoplastic tangent modulus

$D_{i j} \quad$ Rate of deformation

$\mathbf{f}^{\text {ext }} \quad$ External force vector

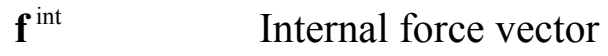

$f_{s} \quad$ Flux of convective velocity

$\mathbf{F}_{\lim } \quad$ Limiting force

$\mathbf{F}_{\text {norm }} \quad$ Normal force

$\mathbf{F}^{n} \quad$ Frictional force at time $n$ 


\begin{tabular}{|c|c|}
\hline $\mathbf{F}_{\text {trial }}$ & Trial force \\
\hline $\mathbf{F}_{y}$ & Yield force \\
\hline$I$ & Nodal number \\
\hline$k$ & Strength coefficient \\
\hline $\mathbf{K}$ & Interface stiffness \\
\hline $\mathbf{K}^{\rho}$ & Divergence matrix for mass density \\
\hline $\mathbf{L}$ & Generalized convective matrix for velocity \\
\hline $\mathbf{L}^{\rho}$ & Transport matrix for mass density \\
\hline $\mathbf{L}^{\sigma}$ & Generalized convective matrix for stress \\
\hline $\mathbf{M}$ & Generalized mass matrix for velocity \\
\hline $\mathbf{M}^{\rho}$ & Capacitance matrix for mass density \\
\hline $\mathbf{M}^{\sigma}$ & Generalized mass matrix for stress \\
\hline$n$ & Hardening exponent \\
\hline $\mathbf{n}^{e}$ & Outward normal to the boundary of element $e$ \\
\hline$n_{j}$ & Outward normal to boundary $\Gamma_{t_{i}}$ \\
\hline$N_{I}$ & Trial shape functions for velocity \\
\hline $\bar{N}_{I}$ & Test shape functions for velocity \\
\hline$N_{I}^{\rho}$ & Trial shape functions for density \\
\hline $\bar{N}_{I}^{\rho}$ & Test shape functions for density \\
\hline$N_{I}^{\sigma}$ & Trial shape functions for the Cauchy stress \\
\hline $\bar{N}_{I}^{\sigma}$ & Test shape functions for the Cauchy stress \\
\hline$N_{s}$ & Total number of sides of element $e$ \\
\hline
\end{tabular}




\begin{tabular}{|c|c|}
\hline$q_{i}$ & Heat flux per unit area \\
\hline$S R C$ & Strain rate parameter \\
\hline$S R P$ & Strain rate parameter \\
\hline $\bar{t}_{i}$ & Boundary tractions \\
\hline $\mathbf{u}$ & Material displacement \\
\hline$\hat{\mathbf{u}}$ & Mesh displacement \\
\hline $\mathbf{v}$ & Material velocity \\
\hline $\mathbf{v}_{I}(t)$ & Material velocity of node $I$ \\
\hline $\mathbf{v}_{r e l}$ & Relative velocity of the surface in contact \\
\hline$\hat{\mathbf{v}}$ & Mesh velocity \\
\hline $\bar{v}_{i}$ & Boundary velocities \\
\hline$\hat{\mathbf{v}}_{I}(t)$ & Mesh velocity of node $I$ \\
\hline $\mathbf{w}$ & Material velocity in the ALE domain \\
\hline $\mathbf{x}$ & Spatial coordinates \\
\hline $\mathbf{x}_{I}(t)$ & Motion of node $I$ \\
\hline $\mathbf{X}$ & Material coordinates \\
\hline $\mathbf{z}$ & Generalized stress vector \\
\hline$\chi$ & ALE coordinates \\
\hline$\chi_{I}$ & ALE coordinates of node $I$ \\
\hline$\delta v_{i}$ & Test function for velocity \\
\hline$\delta \rho$ & Test function for mass density \\
\hline$\delta \sigma_{i j}$ & Test function for stress \\
\hline
\end{tabular}




\begin{tabular}{ll}
$\varepsilon_{y p}$ & Elastic strain to yield \\
$\bar{\varepsilon}^{p}$ & Effective plastic strain (logarithmic) \\
$\phi$ & Field variable which represents density, velocity and stress \\
$\phi_{n+1}$ & Final solution \\
$\phi_{n+1}^{C L}$ & Field variable in the element contiguous to element $e$ \\
$\phi_{n+1}^{L}$ & Solution from the Lagrangian step \\
$\phi_{s, n+1}^{L}$ & Field variable along the side $s$ of element $e$ \\
$\kappa$ & Coefficient of viscous friction \\
$\mu$ & Frictional coefficient \\
$\mu_{s}$ & Static frictional coefficient \\
$\mu_{d}$ & Dynamic frictional coefficient \\
$\rho$ & Mass density \\
$\rho_{I}(t)$ & Mass density of node $I$ \\
$\sigma_{i j}^{\nabla}$ & Heat source per unit volume \\
$\sigma_{j}$ & Internal energy per unit volume \\
$\sigma_{j i}$ & Cauchy stress \\
\hline int & Objective rate of the Cauchy stress \\
\hline &
\end{tabular}




$\begin{array}{ll}\xi^{e} & \text { Element coordinates of element } e \\ \xi_{c r i t} & \text { Critical damping } \\ \xi, \eta, \zeta & \text { Curvilinear coordinates } \\ \Gamma_{v_{i}} & \text { Velocity boundary of the spatial domain } \\ \Gamma_{t_{i}} & \text { Traction boundary of the spatial domain } \\ \Gamma^{e} & \text { Boundary of } \Omega^{e} \\ \Omega & \text { Spatial domain } \\ \Omega^{e} & \text { Spatial domain of element } e \\ \Phi(\chi, t) & \text { Mapping function from the ALE domain to the spatial domain } \\ \varphi(\mathbf{X}, t) & \text { Mapping function from the material domain to the spatial domain } \\ \Psi(\chi, t) & \text { Mapping function from the ALE domain to the material domain }\end{array}$

